\title{
Fibers add flavor. Part II. 5d SCFTs, gauge theories, and dualities
}

\author{
Fabio Apruzzi, ${ }^{a}$ Craig Lawrie, ${ }^{b}$ Ling Lin, ${ }^{b}$ Sakura Schäfer-Nameki ${ }^{a}$ and ${\text { Yi-Nan } \text { Wang }^{b}}^{b}$ \\ ${ }^{a}$ Mathematical Institute, University of Oxford, \\ Andrew-Wiles Building, Woodstock Road, Oxford, OX2 6GG, U.K. \\ ${ }^{b}$ Department of Physics and Astronomy, University of Pennsylvania, \\ Philadelphia, PA 19104, U.S.A.
}

\begin{abstract}
In $[1,2]$ we proposed an approach based on graphs to characterize 5 d superconformal field theories (SCFTs), which arise as compactifications of $6 \mathrm{~d} \mathcal{N}=(1,0)$ SCFTs. The graphs, so-called combined fiber diagrams (CFDs), are derived using the realization of 5d SCFTs via M-theory on a non-compact Calabi-Yau threefold with a canonical singularity. In this paper we complement this geometric approach by connecting the CFD of an SCFT to its weakly coupled gauge theory or quiver descriptions and demonstrate that the CFD as recovered from the gauge theory approach is consistent with that as determined by geometry. To each quiver description we also associate a graph, and the embedding of this graph into the CFD that is associated to an SCFT provides a systematic way to enumerate all possible consistent weakly coupled gauge theory descriptions of this SCFT. Furthermore, different embeddings of gauge theory graphs into a fixed CFD can give rise to new UV-dualities for which we provide evidence through an analysis of the prepotential, and which, for some examples, we substantiate by constructing the M-theory geometry in which the dual quiver descriptions are manifest.
\end{abstract}

KEYwords: F-Theory, M-Theory, Supersymmetric Gauge Theory, Supersymmetry and Duality

ArXiv EPrint: 1909.09128 


\section{Contents}

1 Introduction 1

2 Coulomb phases, box graphs, and 5d SCFTs 5

2.1 The Coulomb branch of $5 \mathrm{~d}$ gauge theories 5

$\begin{array}{lll}2.2 & \text { Phases for } 5 \text { d gauge theory via box graphs } & 7\end{array}$

2.3 Box graphs and flavor symmetries 9

2.4 Intermezzo: gauge theory phases for rank one 5d SCFTs 11

3 Gauge theory phases and box graphs for arbitrary quivers 13

$\begin{array}{lll}3.1 & \text { Flavor-equivalence classes and box graph CFDs } & 15\end{array}$

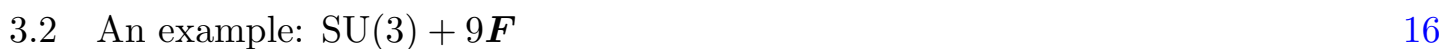

$\begin{array}{lll}3.3 & \text { From box graphs to 5d gauge theories and SCFTs } & 19\end{array}$

3.4 Complex representations $\quad 22$

$\begin{array}{ll}3.5 & \text { Quaternionic representations } \\ 3.6 & 25\end{array}$

$\begin{array}{lll}3.6 & \text { Real representations } & 29\end{array}$

3.7 Flavor-equivalence classes for quiver gauge theories 31

4 Weakly-coupled descriptions from CFDs 34

4.1 Recap: CFDs 36

$\begin{array}{lll}4.2 & \text { Constraints on quiver gauge theories } & 37\end{array}$

$\begin{array}{lll}\text { 4.3 Consistent quivers for the rank one E-string } & 39\end{array}$

4.4 Consistent quivers for $\left(D_{k}, D_{k}\right)$ minimal conformal matter 41

4.5 Consistent quivers for rank two "Model 3" 43

4.6 Consistent quivers for rank two "Model 4" 44

4.7 Consistent quivers for $\left(E_{6}, E_{6}\right)$ minimal conformal Matter 45

4.8 Consistent quivers for $\left(E_{7}, E_{7}\right)$ minimal conformal matter 47

4.9 Consistent quivers for $\left(E_{8}, E_{8}\right)$ minimal conformal matter $\quad 50$

4.10 Consistent quivers for $\left(E_{8}, \mathrm{SU}(2 k+1)\right)$ minimal conformal matter 52

4.11 Consistent quivers for $\left(E_{8}, \mathrm{SU}(2 k)\right)$ minimal conformal matter 53

5 Bootstrapping CFDs $\quad \mathbf{5 5}$

5.1 Constraining marginal CFDs of single gauge node theories $\quad 55$

$\begin{array}{lll}5.2 & \mathrm{SU}(2 n)_{0}+2 \boldsymbol{A} \boldsymbol{S}+8 \boldsymbol{F} & 56\end{array}$

$\begin{array}{lll}5.3 \mathrm{SU}(2 n+1)_{0}+2 \boldsymbol{A} \boldsymbol{S}+8 \boldsymbol{F} & 60\end{array}$

$5.4 \mathrm{SU}(4)_{0}+2 \boldsymbol{A} \boldsymbol{S}+8 \boldsymbol{F}$ and $\left(E_{7}, \mathrm{SO}(7)\right)$ conformal matter 64

6 Descendants and dualities $\quad 67$

6.1 Descendants and dualities for minimal $\left(E_{6}, E_{6}\right)$ conformal matter $\quad 67$

$\begin{array}{lll}\text { 6.1.1 An asymmetric quiver } & 67\end{array}$

$\begin{array}{lll}\text { 6.1.2 Maximal quivers } & 69\end{array}$

$\begin{array}{lll}\text { 6.1.3 Dualities between different quivers } & 71\end{array}$ 
6.2 Descendants and dualities for minimal $\left(E_{7}, E_{7}\right)$ conformal matter 72

6.3 Descendants and dualities for minimal $\left(E_{7}, \mathrm{SO}(7)\right)$ conformal matter $\quad 74$

6.4 Dualities for the marginal theory: $\mathrm{SU}(2 n+1)_{0}+2 \boldsymbol{A} \boldsymbol{S}+8 \boldsymbol{F} \quad 75$

6.5 Dualities for the marginal theory: $\mathrm{SU}(2 n)_{0}+2 \boldsymbol{A} \boldsymbol{S}+8 \boldsymbol{F}$

$\begin{array}{lll}\mathbf{7} & \text { Fibers from Coulomb branch phases } & \mathbf{7 6}\end{array}$

7.1 5d SCFTs and M-theory on elliptic Calabi-Yau threefolds $\quad 76$

$\begin{array}{lll}7.2 & \text { Fibers from box graphs } & 77\end{array}$

$\begin{array}{lll}7.3 & \text { Fiber reconstruction for rank one SCFTs } & 80\end{array}$

7.4 Classification of rank 2 theories from box graphs 86

$\begin{array}{lll}\text { 7.4.1 } & \text { Marginal theories and box graphs } & 87\end{array}$

$\begin{array}{lll}\text { 7.4.2 Phases to fibers } & 87\end{array}$

8 SCFTs/CFDs from box graphs $\quad \mathbf{9 0}$

8.1 Box graphs to superconformal flavor symmetry and CFDs 91

$\begin{array}{lll}\text { 8.2 Rank two CFDs from box graphs } & 92\end{array}$

9 Conclusions and outlook $\quad 94$

A Coulomb branch and reduced CFDs for rank two 5d SCFTs $\quad 96$

A.1 Descendants of rank 2 E-string and $\left(D_{5}, D_{5}\right)$ conformal matter 96

$\begin{array}{lll}\text { A.2 } & \mathrm{SU}(3) \text { on a }(-1) \text {-curve with } 12 \text { hypermultiplets } & 97\end{array}$

$\begin{array}{lll}\text { A.3 } & \mathrm{SU}(3) \text { on a }(-2) \text {-curve with } 6 \text { hypermultiplets } & 101\end{array}$

B Flavor symmetry enhancements for $\mathrm{SU}(N)_{k}+1 A S+N_{f} F \quad 104$

$\begin{array}{lr}\text { C Details for geometric resolutions } & 106\end{array}$

$\begin{array}{ll}\text { C.1 Rank two E-string } & 106\end{array}$

C.2 Resolutions with different rulings for $E_{6} \times E_{6}$ conformal matter 111

$\begin{array}{lll}\text { C.3 Marginal geometry for }\left(E_{7}, \mathrm{SO}(7)\right) \text { minimal conformal matter } & 118\end{array}$

\section{Introduction}

Supersymmetric gauge theories are an ideal setup to explore strongly-coupled aspects of quantum field theories. In less than five dimensions they are renormalizable theories, whereas in higher (five and six) dimensions they can be effective descriptions at low energy. Thanks to the additional structure provided by supersymmetry, one can study features such as electric-magnetic dualities or renormalization group flows even in the absence of perturbative control at all energy scales. In practice, they can be used to probe strongly coupled regimes, giving insights about the non-perturbative dynamics of quantum field theories.

A particularly interesting class are five dimensional (5d) $\mathcal{N}=1$ gauge theories, which can be low energy descriptions of superconformal field theories (SCFTs). More specifically, by studying the space of one-loop corrected couplings, parametrized by the Coulomb branch, one can argue necessary conditions for the existence of a strongly coupled ultraviolet (UV) fixed point [3]. Another motivation to study these theories at present is recent 
progress in 6d SCFTs, where it is believed that a full classification of all UV-complete supersymmetric theories exists [4-7]. Indeed, recent works [1, 2, 8-14] have suggested that all $5 \mathrm{~d} \mathcal{N}=1 \mathrm{UV}$-complete theories arise from appropriate circle reductions, possibly with holonomies for the global symmetries of $6 \mathrm{~d}$ theories, thus conjecturing a classification of $5 \mathrm{~d}$ theories.

Like their six dimensional cousins, 5d SCFTs are inherently strongly coupled. In the absence of a Lagrangian description, methods from or inspired by string theory have proved to be invaluable in their studies $[3,15-26]$. One of the important lessons we have learned from these methods is that many different $5 \mathrm{~d}$ gauge theories can have the same SCFT as UV-completion, thus being $U V$-dual (or, simply, dual) to each other. Another crucial aspect, which manifests itself at strong coupling, is that the flavor symmetry of the gauge theory description can enhance at the UV-fixed point. This fact is due to the presence of non-perturbative instanton operators, which quantum mechanically enhance the classical flavor symmetry at the SCFT point. The state-of-the-art method to calculate the SCFT's flavor symmetry typically involves a localization computation in field theory or a description in terms of 5-brane webs [27-38].

In recent works $[1,2]$ we proposed an alternative approach that arose out of the wellestablished geometric engineering via M-theory on a non-compact Calabi-Yau threefolds [8$10,12,39-42]$. One of the key insights of $[1,2]$ is that there is a succinct description of the CFT data in terms of graphs, and transitions between graphs correspond to mass deformations and subsequent RG-flows. These graphs, the combined fiber diagrams (CFDs), not only capture how 5d SCFTs are interconnected, but more importantly, they encode the strongly-coupled flavor symmetry of the UV fixed point SCFT, as well as the BPS states.

The central idea connecting the CFDs and 5d SCFTs is as follows: given a marginal ${ }^{1}$ theory whose UV completion is a given 6d SCFT, all its descendant 5d SCFTs are obtained via mass deformations and RG-flows. These field theoretic transitions can be encoded via simple graph-theoretic operations on the CFDs, that is associated with each SCFT, and from which the complete tree of descendants is obtained straightforwardly. The CFDs can be thought of as characterizing physically inequivalent M-theory geometries, which are in general non-flat resolutions (see [43] for an in-depth discussion) of the non-compact elliptic Calabi-Yau threefold underlying the F-theory realization of the given 6d SCFT.

The goal of the present paper is to put this into the context of a gauge theoretic description. In particular, we connect the Coulomb branch phases of the effective theory [40], described in terms of representation-theoretic graphs [44], to the CFD-characterization of the SCFT limit. The focus here is three-fold:

1. Constraining the possible weakly-coupled gauge theory descriptions of a 5d SCFT given in terms of a CFD,

2. Derivation and constraints on UV-dualities using the CFD description,

3. Bootstrapping CFDs for marginal theories, in cases where no CFD-description is known, but weakly-coupled descriptions are available.

\footnotetext{
${ }^{1}$ In this paper, we will consider theories that are both marginal and have a $6 \mathrm{~d}$ UV fixed point. As such we will use the notation interchangeably, however see [9] for exceptions.
} 
Geometry and CFDs. Before expanding on these points, let us briefly recapitulate the relation between the geometry of non-compact elliptically fibered Calabi-Yau threefolds with canonical singularities and 5d SCFTs. The 5d SCFT arising from the canonical singularity can be identified by virtue of the M-/F-theory duality [45-47] with circle reductions of the $6 \mathrm{~d}$ theory realized in F-theory, including possible holonomies in the flavor symmetry. The resolutions of the canonical singularities consist of a collection of intersecting compact surfaces, and, field theoretically, their volumes parametrize the Coulomb branch of the theory. These surfaces shrink to a point in the singular limit, which corresponds to the UV fixed point. When these divisors are ruled ( $\mathbb{P}^{1}$ fibered over a curve) and intersect along sections of the rulings, the collection of surfaces may be collapsed to a curve of singularities after the ruling curves are collapsed to zero volume. The additional light states appearing from M2-branes wrapping the fibers of these rulings give rise to a gauge theory. Finally, when a bouquet of surfaces shrinks to a collection of intersecting curves of singularities, the underlying low energy description is generically given by a quiver gauge theory.

The starting point of our analysis is the so-called $5 \mathrm{~d}$ marginal theory, which is obtained by taking the $6 \mathrm{~d}$ theory compactified on a circle (or alternatively M-theory on the same elliptically fibered Calabi-Yau), without any holonomy for the flavor symmetry. This theory usually has an effective gauge theory description, which has a 6d SCFT as its UV fixed point. Starting from the marginal theory we can turn on mass deformations. This procedure allows one to obtain all descending 5d SCFTs corresponding to partial blow-downs of the fully resolved geometry, and these descendants can be enumerated combinatorially. From the gauge theory point of view this procedure corresponds to decoupling matter hypermultiplets, whereas from a strongly coupled perspective, the resulting descendants are the end-products after renormalization-group (RG) flows that are triggered by the mass deformations. The set of descendant 5d SCFTs linked by RG flow leads to a connected tree of theories. One of the main advantages of our approach is that the complete tree of descendants is obtained from the CFD associated to the marginal 5 d theory by simple operations on the graphs, and can be fully automated.

A complete classification of all 5d SCFTs that descend from 6d SCFTs by circlereductions requires as input the set of all marginal theories, the associated CFDs (usually computed by resolving the geometry). From this the procedure determines the descendants uniquely. The single gauge node marginal theories were determined in [9], and we will discuss this class of theories in the present paper. Another class that already featured prominently in $[1,2]$ are $5 \mathrm{~d}$ theories descending from $6 \mathrm{~d}$ minimal conformal matter theories [48]. One of the outputs of this paper are proposals for weakly coupled descriptions of these theories, as well as dualities among these. In many instances we can substantiate these weakly coupled descriptions as well as dualities by determining the associated rulings in the resolved elliptic Calabi-Yau geometry.

Gauge theories, Coulomb branches, dualities and CFDs. The strength of the approach that we proposed in $[1,2]$ lies in its combinatorial nature, which at the same time captures not only the network of 5d SCFTs that descend from a $6 \mathrm{~d}$ theory, but also the flavor symmetry of the UV-fixed point. While the latter is often enhanced compared 
to the classical gauge theory descriptions, our previous discussions were focused primarily on the SCFT itself.

In this work, we extend the scope of this approach by explicitly studying the effective gauge descriptions of the SCFTs.

A central tool to achieve this is a representation-theoretic object, the box graph, introduced in [44], which captures all Coulomb branch phases of a given 5d gauge theory. The Coulomb branch on the other hand is intimately linked to the relative Mori cone of the elliptic Calabi-Yau threefold $[44,49-51]^{2}$ The box graphs fully encode the sets of consistency conditions on the Coulomb branch of a gauge theory with matter, where the matter classically transforms under a flavor group, $G_{\mathrm{F}, \mathrm{cl}}$ as well as the gauge group $G_{\text {gauge }}$. In particular, we couple the gauge theory to a non-trivial background connection for the flavor symmetry, by weakly gauging it. This leads to a set of cone inequalities not only for the Coulomb branch parameters, but also to consistency conditions for the possible masses of the flavor hypermultiplets. This description is very convenient, since the mass deformations of the gauge theory are characterized in terms of simple operations on the box graphs. In brief, a Coulomb branch phase is given in terms of a representation graph (encoding the transformation of the matter under both the gauge and classical flavor symmetries), as well as a sign-assignment or decoration, which specifies the Coulomb branch phase.

We will define a class of graphs, which characterize $5 \mathrm{~d}$ gauge theories: they encode the classical flavor symmetry of the gauge theory. These graphs, the box graph CFDs ( $B G$-CFDs), encode equivalence classes of Coulomb branch phases, which all carry the same classical flavor symmetry. We first determine these for all possible gauge groups and matter contents. From this we can then build the corresponding BG-CFDs for quivers.

We then use these to constrain the possible weakly coupled gauge theory descriptions of a given CFD (starting with the CFD for a marginal 5d theory, but also for all its descendants), by embedding the BG-CFDs into the CFDs. This, for instance, implies that for rank two 5d SCFTs, the known weakly-coupled descriptions are a comprehensive list. More interestingly, however, we can predict new weakly coupled gauge theory or quiver descriptions for theories where only few such descriptions exist, such as the $\left(E_{n}, E_{n}\right)$ minimal conformal matter theories as well as $\left(E_{8}, \mathrm{SU}(n)\right)$, and $\left(E_{7}, \mathrm{SO}(7)\right)$ conformal matter. In all these cases a geometric derivation of the marginal CFD exists. Another implication of the relation between CFDs and BG-CFDs is that we can predict a large class of new dualities, i.e., gauge theories or quivers, which have the same UV fixed point.

There are $6 \mathrm{~d}$ theories, where no known elliptic fibration in terms of a Weierstrass model for the fully singular geometry exists. In such instances we can turn the arguments around and use our approach to constrain the marginal CFD, by using known gauge theory descriptions as well as flavor symmetry enhancements of the $5 \mathrm{~d}$ descendants.

The plan of the paper is as follows: to set the stage, we give a lightning review of $5 \mathrm{~d}$ Coulomb branches in the language of box graphs in section 2. We then propose how to use this approach to study 5d gauge/quiver theories with matter and introduce the concept

\footnotetext{
${ }^{2}$ This structure has played an important role also F-theory on elliptic fourfolds and fivefolds in the context of $G_{4}$-fluxes and chiralitiy [44, 49-55].
} 
of flavor equivalence classes of Coulomb branch phases (or box graphs) and the BG-CFDs in section 3. This is done for all types of gauge theories and matter in $5 \mathrm{~d}$ that have an SCFT in the UV. In section 4 we use this to constrain the weakly-coupled descriptions of marginal theories for all rank two $5 \mathrm{~d}$ theories, as well as the marginal theories associated to minimal conformal matter theories of type $\left(D_{k}, D_{k}\right),\left(E_{n}, E_{n}\right),\left(E_{8}, \mathrm{SU}(n)\right)$. For all these models, we computed the CFDs of the marginal theories from geometry. In section 5.1 we turn this around and discuss theories, which do not have a known description in terms of a fully singular Tate or Weierstrass model. Nevertheless, we find that we can bootstrap the candidate marginal CFD using the information about known weakly coupled descriptions, and their flavor symmetry enhancements. Interestingly, these are precisely the theories that are relatively easily accessible using other methods (such as 5-brane webs), whereas for the models where we can determine the marginal CFD from geometry, the weakly coupled descriptions are often somewhat sparse (e.g., the $\left(E_{n}, E_{n}\right)$ conformal matter theories).

Descendant 5d SCFTs and dualities among weakly coupled descriptions that can be infered from the CFDs are the topic of section 6 . We first discuss two cases where the dualities have a geometric underpinning: the marginal theories from $\left(E_{6}, E_{6}\right)$ and $\left(E_{7}, \mathrm{SO}(7)\right)$ conformal matter and their descendants. We propose new quiver descriptions for these theories as well as the complete network of descendants and their gauge theory descriptions, whenever these exist. This is backed by a geometric analysis in appendix C.

Finally, in sections 7 and 8 we return to geometry to tie up some loose ends, and show how all three strands of our analysis - the resolved elliptic Calabi-Yau, the CFDs and the gauge theory Coulomb branch phases - are connected. In particular we quantify how the gauge theory description needs to be supplemented to see, for instance, the full superconformal flavor symmetry manifest in geometry. We conclude with a summary and outlook in section 9. In appendix A, we summarize all gauge theory phases (and associated BG-CFDs) for the rank two 5d theories. Appendix C contains details of the resolutions for marginal and descendant theories.

\section{Coulomb phases, box graphs, and 5d SCFTs}

In this section, we summarize some of the basic ingredients that will be combined in this paper. For starters, we discuss the structure of the Coulomb branch of $5 \mathrm{~d}$ gauge theories supplementing the material in Part I [2], where some aspects of this were already discussed. Here our focus will be to characterize the Coulomb branch of a 5 d gauge theory with matter, using the underlying representation-theoretic structure, based on the classic [40] as well as the box graph description in [44].

\subsection{The Coulomb branch of $5 \mathrm{~d}$ gauge theories}

The Coulomb branch of a $5 \mathrm{~d} \mathcal{N}=1$ supersymmetric gauge theory coupled to matter can have an intricate structure. Let us consider a gauge theory with reductive gauge group, $G_{\text {gauge }}$, written as a product

$$
G_{\text {gauge }}=\prod_{i} G_{i}^{r_{i}} \times \mathrm{U}(1)^{r_{A}}
$$


where $G_{i}$ are simple groups, the superscript indicates the rank, and further

$$
r_{A}=r-\sum_{i} r_{i}
$$

is the rank of the abelian subgroup transverse to the Cartan subgroup of the non-abelian factors. In this notation the Coulomb branch is isomorphic to

$$
\left(\prod_{i} \mathbb{R}^{r_{i}} / W_{G_{i}}\right) \times \mathbb{R}^{r_{A}},
$$

where $W_{G_{i}}$ is the Weyl group of $G_{i}$. The quotient is the Weyl chamber, defined by

$$
\mathcal{C}_{i}=\mathbb{R}^{r_{i}} / W_{G_{i}}
$$

and it has the structure of a cone. Thus the grossest feature of the Coulomb branch of $5 \mathrm{~d}$ supersymmetric gauge theory is that it is a collection of cones; this property comes only from considering the gauge group itself, and this structure is further refined in a theory that also incorporates matter [40].

We can choose a basis such that the $\mathcal{C}_{i}$ are the fundamental Weyl chambers of the $G_{i}$. Let $\alpha_{j}^{(i)}$ be the positive simple roots of $G_{i}$, then we can write ${ }^{3}$

$$
\mathcal{C}_{i}=\left\{\phi \in \mathbb{R}^{r_{i}} \mid\left\langle\phi, \alpha_{j}^{(i)}\right\rangle>0 \text { for all } j\right\}
$$

Consider now a hypermultiplet, $H$, transforming in a representation $\boldsymbol{R}$ of $G$. On the Coulomb branch of the theory the gauge group is broken to $\mathrm{U}(1)^{r}$. The hypermultiplets transform as a collection of $\operatorname{dim}(\boldsymbol{R})$ hypermultiplets under the $\mathrm{U}(1)^{r}$ in the representation defined by the weights of $\boldsymbol{R}$. Let us, for the moment, consider a representation $\boldsymbol{R}_{i}$ of $G_{i}$ and highlight the induced structure on the Coulomb branch from the presence of these hypermultiplets. A hypermultiplet carrying the charges under $\mathrm{U}(1)^{r}$ corresponding to the weight $\lambda$ of $\boldsymbol{R}_{i}$ becomes massless at the point in the Coulomb branch where

$$
\langle\phi, \lambda\rangle=0
$$

It is easy to see that for each $\lambda$ in $\boldsymbol{R}_{i}$ this gives rise to a wall inside the Coulomb branch, and along this wall there exist additional massless hypermultiplets. We can then describe the subchambers, or subwedges, of $\mathcal{C}$ as defined by these walls. A phase of the gauge theory is defined as a non-empty subwedge of the Coulomb branch such that each

$$
\left\langle\phi, \lambda_{I}\right\rangle \text { has a definite sign for each } \lambda_{I} \in \boldsymbol{R} .
$$

Determining the phase structure of the Coulomb branch involves determining these subwedges, and the adjacency relations between them.

\footnotetext{
${ }^{3}$ Appropriate care must be taken here with respect to weight and coweight lattices, which we are pairing between.
} 


\subsection{Phases for 5d gauge theory via box graphs}

It is useful to formulate the problem of finding the Coulomb branch phases of a $5 \mathrm{~d}$ gauge theory in terms of so-called Box Graphs [44], which provide a succinct combinatorial way to list all phases.

The set of weights of each irreducible representation $\boldsymbol{R}$ of a group $G$ is generated by starting with a highest weight and from that highest weight one repeatedly subtracts positive simple roots, following a simple prescription. That is, if $\lambda$ is a weight of $\boldsymbol{R}$ then it can be written as the linear combination

$$
\lambda^{\mathrm{hw}}-\sum_{j} n_{j} \alpha_{j}
$$

where the $n_{j}$ are non-negative integers and $\lambda^{\text {hw }}$ is the highest weight of $\boldsymbol{R}$. This action of generating all the weights of a representation from the highest weight forms the weight diagram [56] of the representation. For an irreducible representation the weight diagram is a connected directed graph where the nodes are the weights of the representation and there exists an edge between two nodes if the two weights differ only by a single positive simple root. We will use a particular presentation of this weight diagram, as explained in the following definition.

Definition 2.1. An undecorated box graph is a graphical depiction of the weight diagram [56] for a representation $\boldsymbol{R}$ for a Lie algebra $\mathfrak{g}$. Each weight of $\boldsymbol{R}$ is represented by a box, and if two weights differ by the addition of a single simple positive root of $\mathfrak{g}$ then their boxes are adjacent.

As we have discussed above, each phase of the Coulomb branch of a $5 \mathrm{~d} \mathcal{N}=1 G_{\text {gauge }}$ gauge theory with matter transforming in a representation $\boldsymbol{R}$ of $G_{\text {gauge }}$ is specified by the signs of $\langle\phi, \lambda\rangle$ for each $\lambda \in \boldsymbol{R}$. We mark the sign assignment for the phase onto the undecorated box graph for the weight diagram as in the following definition.

Definition 2.2. A (decorated) box graph is an assignation of \pm signs to each weight, $\lambda$, represented in an undecorated box graph such that

$$
\{ \pm\langle\lambda, \phi\rangle>0\} \cap\left\{\left\langle\alpha_{i}, \phi\right\rangle>0\right\}
$$

has non-zero solutions for $\phi$. We will write $\lambda^{ \pm}$for the weight appearing in the decorated box graph together with the assigned sign of $\langle\lambda, \phi\rangle$. In this way one can see that a decorated box graph is defined such that it corresponds to a non-empty phase of the Coulomb branch of a $5 \mathrm{~d}$ gauge theory with gauge algebra $\mathfrak{g}$ and matter transforming in the representation $\boldsymbol{R}$.

In practice we will represent the positive and negative weights by blue/yellow boxes.

It is clear to see that if the weight $\lambda$ is assigned the sign + in the decorated box graph, then all weights $\widetilde{\lambda}$ such that

$$
\lambda=\widetilde{\lambda}-n_{j} \alpha_{j},
$$

for $n_{j}$ non-negative must also be assigned + . Explicitly, there only exists a non-zero value of $\phi$ solving

$$
\langle\phi, \lambda\rangle=\langle\phi, \widetilde{\lambda}\rangle-n_{j}\left\langle\phi, \alpha_{j}\right\rangle
$$


subject to the assumptions that

$$
\langle\phi, \lambda\rangle>0, \quad\left\langle\phi, \alpha_{j}\right\rangle>0,
$$

if one also has

$$
\langle\phi, \widetilde{\lambda}\rangle>0 .
$$

Similarly, one can show that if $\lambda$ is assigned a $-\operatorname{sign}$ in the decorated box graph the all weights $\widetilde{\lambda}$ which satisfy

$$
\widetilde{\lambda}=\lambda-n_{j} \alpha_{j}
$$

again for $n_{j}$ non-negative, must also be assigned a - sign.

As we have just seen, not all the weights of the representation can be assigned signs independently, in fact it is usually the case that once the signs are associated to a few weights the signs of all the other weights follows of necessity. We will refer to these weights as extremal, and they are defined as follows.

Definition 2.3. A weight $\lambda^{+}$in a decorated box graph is extremal if there does not exist a weight $\widetilde{\lambda}^{+}$in the decorated box graph such that

$$
\widetilde{\lambda}=\lambda-n_{j} \alpha_{j},
$$

for $n_{j}$ non-negative. Similarly a weight $\lambda^{-}$is extremal if there does not exist a $\widetilde{\lambda}^{-}$such that

$$
\lambda=\widetilde{\lambda}-n_{j} \alpha_{j},
$$

again for $n_{j}$ non-negative integers.

Definition 2.4. In a decorated box graph a root, $\alpha_{j}$, of the gauge group $G$ is said to split if we have two weights related in the box graph as

$$
\tilde{\lambda}=\lambda-\alpha_{j}
$$

such that $\widetilde{\lambda}$ is assigned the sign - and $\lambda$ is assigned + . For such split roots we can use

$$
\langle\phi, \lambda\rangle-\langle\phi, \widetilde{\lambda}\rangle=\left\langle\phi, \alpha_{j}\right\rangle,
$$

to see that $\left\langle\phi, \alpha_{j}\right\rangle>0$ is automatically satisfied by the sign assignment of $\lambda$ and $\widetilde{\lambda}$. Generally the $\tilde{\lambda}^{+}$and $\lambda^{-}$may be further rewritten as a positive linear combination of the extremal weights and the non-split roots.

The motivation for the previous two definitions is as follows. The signs associated to the non-extremal weights and the split roots are determined from the signs associated to the extremal weights and the non-split roots. In this manner each subwedge for a simple gauge group $G$ and matter in an irreducible representation $\boldsymbol{R}$ can be minimally written as

$$
\mathcal{W}_{i}=\left\{\phi \in \mathbb{R}^{\operatorname{rank}(G)} \mid \pm\left\langle\phi, \lambda_{j}^{\text {extremal }}\right\rangle>0 \text { and }\left\langle\phi, \alpha_{k}^{\text {non-split }}\right\rangle>0 \text { for all } j, k\right\},
$$

where the indices run over all extremal (the \pm is given by the sign of the extremal weight) and non-split roots in the particular subwedge under consideration. In this way we can 
see that it is this restricted set of weights and roots that are the "irreducible" objects generating the subwedge.

In the pictorial decorated box graphs this leads to the following definition of flow rules, which follow directly from the above consistency requirements for the sign assignments in the box graphs:

Definition 2.5. The flow rules state that if we assign the sign + to a weight of an undecorated box graph then we must assign + also to every box up and to the left of that weight. Similarly if we assign - to a particular weight then we must assign - to every weight that is down and to the right. This is captured graphically by
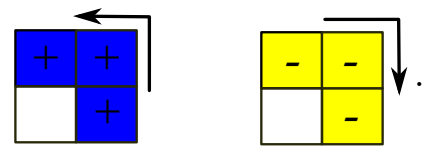

Definition 2.6. A flop transition exists between two decorated box graphs if a single weight differs in assigned sign. These weights are necessarily extremal weights, and a flop transition is changing precisely one sign assignment of an extremal weight. Generally we will be considering representations that contain weights $\lambda$ and $-\lambda$, for example selfconjugate representations, and when we say that a single weight differs in sign we mean that the signs associated to $\lambda$ and $-\lambda$ are swapped. Two Coulomb phases are adjacent inside of the Coulomb branch if the associated box graphs are related by a flop transition.

For a more in depth discussion of box graph and Coulomb phases we refer the reader to [44] and [49-51].

\subsection{Box graphs and flavor symmetries}

Although box graphs are used to characterize the Coulomb branch phases of gauge theories in $5 \mathrm{~d}$ (or $3 \mathrm{~d}$ ) with matter, we can equally apply them to determine the structure of the extended Coulomb branch, of a gauge theory with classical flavor symmetry $G_{\mathrm{F} \text {, cl }}$. Consider a gauge theory with matter in $\left(\boldsymbol{R}, \boldsymbol{R}_{\mathrm{BG}}\right)$ of $G_{\text {gauge }} \times G_{\mathrm{BG}}$. To determine which matter multiplets can be given masses and can be decoupled from the theory, recall that the prepotential has a contribution

$$
\mathcal{F} \supset-\frac{1}{12} \sum_{\boldsymbol{R}_{\mathrm{BG}}} \sum_{\lambda_{\boldsymbol{R}_{\mathrm{BG}}}}\left|\lambda_{i} \phi^{i}+m_{f}\right|^{3},
$$

where $\phi^{i}$ are the scalars in the vector multiplet, which are coordinates on the Coulomb branch, and $m_{f}$ are masses for hypermultiplets. The sum runs over the weights of the representation. Promoting the masses $m_{f}$ as parameters of the Coulomb branch, corresponds to weakly gauging part of the flavor symmetry. In practice, this is equivalent to studying the Coulomb branch, or box graphs, for bifundamental matter of ( $\boldsymbol{R}_{\text {gauge }}, \boldsymbol{R}_{\mathrm{BG}}$ ) of $G_{\text {gauge }} \times G_{\mathrm{BG}}$.

Our strategy will be to determine all phases of the extended Coulomb branch using box graphs, starting with a marginal $5 \mathrm{~d}$ theory, i.e., the gauge theory description of a circlereduction of a $6 \mathrm{~d}$ SCFT. This determines all descendant gauge theories, that can be reached 


\begin{tabular}{|c|c|c|}
\hline Gauge Group & Matter & Flavor Group $G_{\mathrm{F}, \mathrm{cl}}$ \\
\hline $\mathrm{SU}(n \geq 3)$ & $\begin{array}{c}N_{f} \times \text { fund } \\
+N_{a} \times \text { anti-sym } \\
+N_{s} \times \mathbf{s y m}\end{array}$ & $\mathrm{U}\left(N_{f}\right) \times \mathrm{U}\left(N_{a}\right) \times \mathrm{U}\left(N_{s}\right)$ \\
\hline $\mathrm{Sp}(n)$ & $\begin{array}{c}N_{f} \times \text { fund } \\
+N_{a} \times \text { anti-sym }\end{array}$ & $\mathrm{SO}\left(2 N_{f}\right) \times \mathrm{Sp}\left(N_{a}\right)$ \\
\hline $\mathrm{SO}(n)$ & $N_{v} \times$ vector & $\mathrm{Sp}\left(N_{v}\right)$ \\
\hline$G_{2}$ & $N_{7} \times \mathbf{7}$ & $\mathrm{Sp}\left(N_{7}\right)$ \\
\hline$F_{4}$ & $N_{26} \times \mathbf{2 6}$ & $\mathrm{Sp}\left(N_{26}\right)$ \\
\hline$E_{6}$ & $N_{27} \times \mathbf{2 7}$ & $\mathrm{U}\left(N_{27}\right)$ \\
\hline$E_{7}$ & $N_{56} \times \mathbf{5 6}$ & $\mathrm{SO}\left(2 N_{56}\right)$ \\
\hline
\end{tabular}

Table 1. Flavor symmetries of $5 \mathrm{~d}$ gauge theories with simple gauge groups. The matter content has been restricted to representations that allow for the gauge theory to have an honest 5d SCFT limit. For this reason, there is no $E_{8}$ theory with non-trivial matter in this table. Note that for $\mathrm{SU}(n \geq 3)$ gauge theories, the $N_{i}$ hypermultiplets transforming in the (anti-)fundamental of the $\mathrm{SU}\left(N_{i}\right) \subset \mathrm{U}\left(N_{i}\right)$ flavor factor have charges $(-) 1$ under the baryonic $\mathrm{U}(1) \subset \mathrm{U}\left(N_{i}\right)$.

by successively decoupling hypermultiplets. As we shall see in section 3, equivalence classes of box graphs will then characterize all 5d SCFTs that admit a weakly coupled gauge theory description. We will illustrate the box graph approach in section 2.4 with the rank one theories. This class of $5 \mathrm{~d}$ theories, descend from a single marginal theory, which is the dimensional reduction of the rank one E-string.

We list for convenience the flavor group for all possible 5 d gauge theories which can have a non-trivial UV fixed point, ${ }^{4}$ following [40]. For gauge theories with a simple gauge group, the data is summarized ${ }^{5}$ in table 1.

For a quiver gauge theory, consisting of $Q$ gauge factors $G_{I}$, there are $Q-1$ hypermultiplets in the bifundamental of $G_{I} \times G_{I+1}$

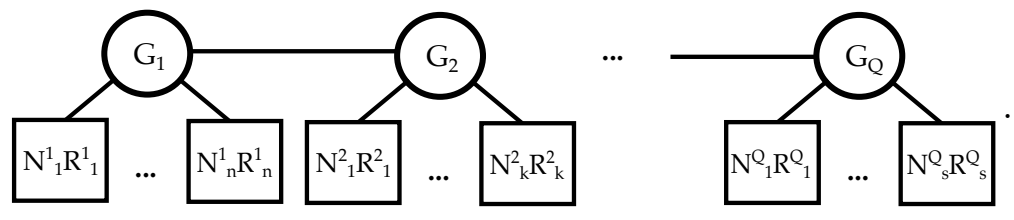

Furthermore, there can be $N_{f_{I}}$ hypermultiplets transforming in a representation $\boldsymbol{R}_{G_{I}}$ of the gauge group $G_{I}$. Typically, one represents such a quiver as a set of nodes, each corre-

\footnotetext{
${ }^{4}$ These are only the matter fields that we consider in this paper. In addition one can have the triple antisymmetric of $\mathrm{SU}(n)$ and the spinor/conjugate spinor of $\mathrm{SO}(n)$ for low ranks of the gauge group; one can also include adjoint matter, which may have a $6 \mathrm{~d}$ fixed point with sixteen supercharges. See [9] for more details.

${ }^{5}$ We note that we rectify the typographical error in [40] whereby the classical flavor symmetry rotating $N_{27}$ fundamental hypermultiplets of $E_{6}$ was written as $\mathrm{SU}\left(N_{27}\right)$ rather than $\mathrm{U}\left(N_{27}\right)$.
} 
sponding to one gauge factor $G_{I}$. Bifundamental matter are showing as lines connecting two nodes, and additional hypermultiplets are indicated by lines attached to a single node.

The global symmetry group can be thought as coming from 3 different contribution:

- Each of the $Q$ gauge group nodes in the quiver has an associated $\mathrm{U}(1)_{T}$.

- For each full hypermultiplet transforming in the bifundamental of $G_{I} \times G_{I+1}$, there will be a baryonic $\mathrm{U}(1)_{B}$, which is an $\mathrm{SU}(2)_{B}$ for an hypermultiplet in the fundamental of two $\mathrm{SU}(2)$ gauge nodes.

- The symmetry rotating the $N_{f_{I}}$ hypers can be read off from the single simple gauge group classical flavor symmetries.

The total global symmetry is a product of these factors.

\subsection{Intermezzo: gauge theory phases for rank one 5d SCFTs}

To illustrate the inner workings of box graphs, let us first consider the simplest example: the rank one theories in 5d, which e.g. arise as dimensional reductions and mass deformations of the $6 \mathrm{~d}$ rank one E-string theory. The marginal theory admits an $\mathrm{Sp}(1)=\mathrm{SU}(2)$ gauge gauge theory description with 8 fundamental flavors [3]

$$
\mathrm{SU}(2)+8 \boldsymbol{F}
$$

The flavor symmetry at weak coupling is then $\mathrm{SO}(16)=G_{\mathrm{BG}}$. In other words, the theory has matter in the $(\mathbf{2}, \mathbf{1 6})$ representation of the $\mathrm{SU}(2)_{\text {gauge }} \times \mathrm{SO}(16)$. This induces a wedge structure on the Coulomb branch of the theory when we weakly gauge the $\mathrm{SO}(16)$, as explained earlier.

To study the different phases of this Coulomb branch, and the corresponding fiber structure, we denote the positive simple roots in the Cartan-Dynkin basis by

$$
\begin{aligned}
& \mathrm{SO}(16): \quad \begin{cases}\alpha_{1}=(0 ; 2,-1,0,0,0,0,0,0), & \alpha_{2}=(0 ;-1,2,-1,0,0,0,0,0) \\
\alpha_{3}=(0 ; 0,-1,2,-1,0,0,0,0), & \alpha_{4}=(0 ; 0,0,-1,2,-1,0,0,0) \\
\alpha_{5}=(0 ; 0,0,0,-1,2,-1,0,0), & \alpha_{6}=(0 ; 0,0,0,0,-1,2,-1,-1) \\
\alpha_{7}=(0 ; 0,0,0,0,0,-1,2,0), & \alpha_{8}=(0 ; 0,0,0,0,0,-1,0,2)\end{cases} \\
& \mathrm{SU}(2): \quad \alpha^{\mathrm{SU}(2)=(2 ; 0,0,0,0,0,0,0,0),}
\end{aligned}
$$

and in this notation the highest weight of the $(\mathbf{2}, \mathbf{1 6})$ is

$$
L_{1,1}=(1 ; 1,0,0,0,0,0,0,0) \text {. }
$$

The undecorated box graph for this representation is given in figure 1. In particular we denote by

$$
L_{i, j}=L_{i}^{\mathbf{2}}+L_{j}^{\mathbf{1 6}}
$$

the sum of fundamental weights of $\mathbf{2}$ and $\mathbf{1 6}$. The simple roots of $\mathrm{SO}(16)$ can be written as

$$
\alpha_{i}=L_{i}^{\mathbf{1 6}}-L_{i+1}^{\mathbf{1 6}}, \quad i=1, \cdots, 7, \quad \alpha_{8}=L_{7}^{\mathbf{1 6}}+L_{8}^{\mathbf{1 6}} .
$$




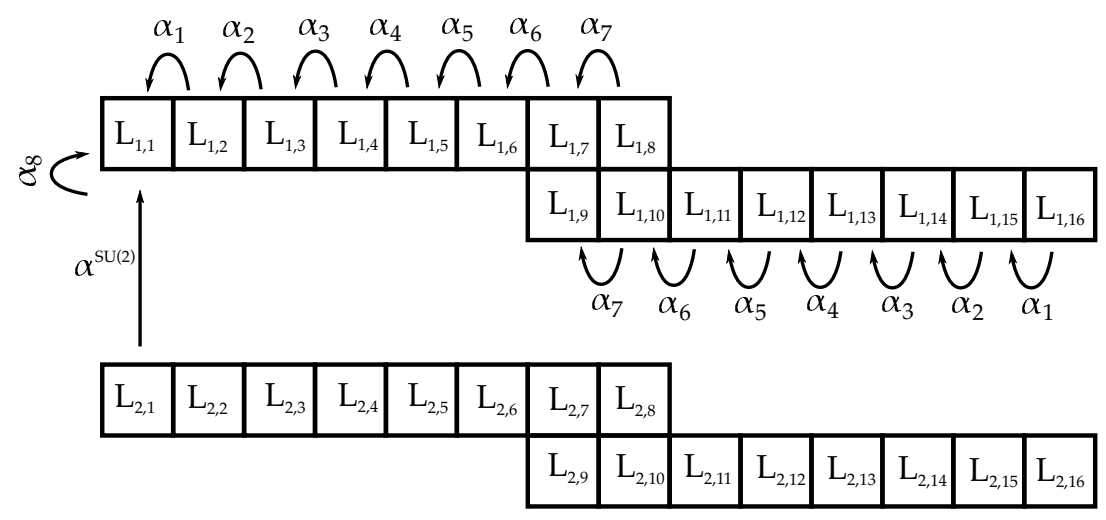

Figure 1. The representation graph (or undecorated box graph) for the $(\mathbf{2}, \mathbf{1 6}$ ) representation of $\mathrm{SU}(2)_{\text {gauge }} \times \mathrm{SO}(16)$. The simple roots of $\mathrm{SO}(16)$ are $\alpha_{i}$, and for $\mathrm{SU}(2)$ gauge the simple root is $\alpha^{\mathrm{SU}(2)}$. The arrows indicate how weights are mapped into each other under the addition of the roots. The weights are $L_{i, j}=L_{i}^{\mathbf{2}}+L_{j}^{\mathbf{1 6}}$, where $L_{i}^{\boldsymbol{R}}$ are the fundamental weights of the representation $\boldsymbol{R}$. The action of the roots is indicated by the arrows. Note that $L_{i+8}^{\mathbf{1 6}}=-L_{9-i}^{\mathbf{1 6}}$ for $i=1, \ldots, 8$.

Starting with the marginal theory, we determine all the consistent phases using the box graphs. The marginal theory is such that all roots of the weakly gauged flavor symmetry $\mathrm{SO}(16)$ are contained in the splitting of the $\mathrm{SU}(2)$. In this case the two 16 representation graphs that are part of the box graph in this case have the same coloring, i.e. coloring the $+/-$ sign assignments in blue/yellow, the decorated box graph associated to this phase is

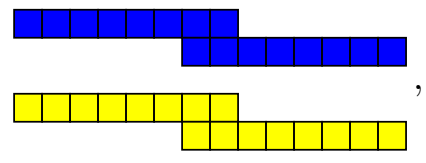

where each box corresponds to a weight as in figure 1. Consistency with the flow rules determine then all further phases, by applying flops. Lets illustrate this by performing one flop on the box graph (2.28). The only extremal weights/boxes are $L_{1,16}$ and $L_{2,1}$ (recall that this representation is self-conjugate so each flop will require changing the color of one box in each of the two 16s). After the flop transition, the new box graph is

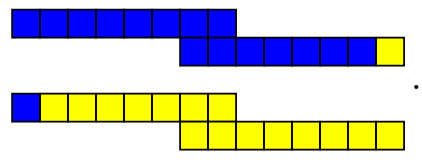

Continuing along these lines results in figure 2 .

This chain is of course precisely the phases of the rank one theories in $5 \mathrm{~d}$ rank as described in the classic works of $[3,39]$, and each flop corresponds to decoupling a fundamental hypermultiplet. In particular, the chain obtained from the box graphs also captures the two possible ways of decoupling the only hypermultiplet of an $\mathrm{SU}(2)+1 \boldsymbol{F}$ theory to flow either to an $\mathrm{SU}(2)_{\theta=0}$ or $\mathrm{SU}(2)_{\theta=\pi}$ theory. Indeed, as we will discuss in section 3 , there is a natural way of interpreting the flop transitions as decoupling of matter multiplets in the limit where we restore the coupling of $G_{\mathrm{BG}}$ to 0 , which also establishes a subgroup of $G_{\mathrm{BG}}$ as the physical weakly coupled flavor symmetry after decoupling the matter. 


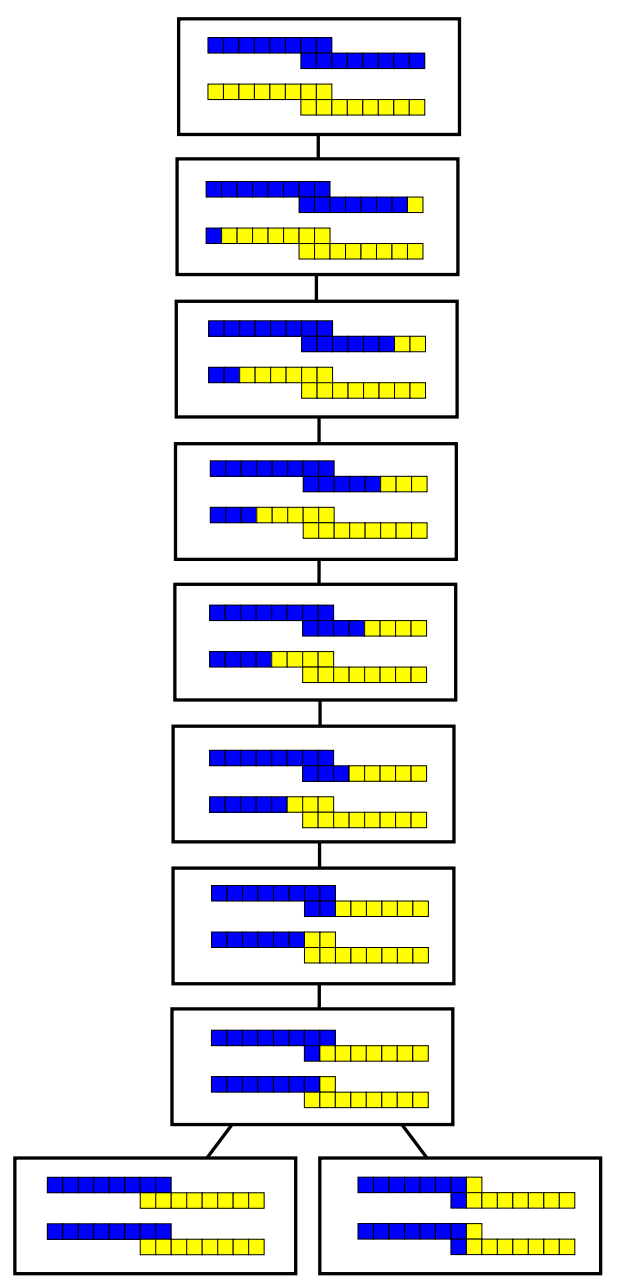

Figure 2. $5 \mathrm{~d}$ rank one theories: box graphs for $(\mathbf{2}, \mathbf{1 6})$ of $\mathrm{SU}(2) \times \mathrm{SO}(16)$, for the marginal theory $\mathrm{SU}(2)+8 \boldsymbol{F}$, which is shown at the top of the tree. Connections indicate 'flop transitions', which in the gauge theory correspond to different phases of the extended Coulomb branch, and from the point of view of the $\mathrm{SU}(2)$ gauge theory, corresponds to decoupling fundamental hypermultiplets.

\section{Gauge theory phases and box graphs for arbitrary quivers}

In this section we will determine the set of 5 d gauge theories that arise as mass deformations of a given theory with gauge group $G_{\text {gauge }}$. We will find that the structure of mass deformations amongst these form a tree of theories with varying matter content charged under $G_{\text {gauge }}$. These theories will not necessarily be distinct, in that they may still admit, what we will call, "discrete dualities"; examples of such dualities are shifting the Chern-Simons level, $k \rightarrow-k$, or shifts of $\theta$-angles in such a way that the theories are identical. More generally, we will also discover such discrete dualities to incorporate simultaneous modification of the number of hypermultiplets coming from different flavor nodes in a quiver.

As we are interested in gauge theories with an SCFT limit, we will focus either on theories where $G_{\text {gauge }}$ is a simple group that appears in table 1 , or on quiver gauge theories where the nodes carry one of these simple factors, together with some of the matter listed 
in the aforementioned table. For low ranks of the gauge group there can be matter fields transforming in more exotic representations, which still flow to an interacting UV fixed point; one example is the triple anti-symmetric representation of $\mathrm{SU}(N)$, for sufficiently small values of $N$. Some of these exceptional gauge theories are pointed out in [9], however we will not consider them further here, as the number of descendant gauge theories can straightforwardly be determined by the methods explained here.

For the simple gauge theories in table 1 the phases of the Coulomb branch has been often studied. For $\mathrm{SU}(N)$ gauge theories the Coulomb phases are enumerated for general $N$ in $[40,44,50]$ and for specific low values of $N$ in $[49,51,54,57-61]$, for $\operatorname{Sp}(N)$ in [40, $44,62]$, for $\mathrm{SO}(N)$ in $[40,44,63,64]$, and for the exceptional cases, $G_{2}, F_{4}, E_{6}$, and $E_{7}$ in [63, 65], [66], [44], and [44, 67], respectively. In this paper we will not require an understanding of the full set of Coulomb phases, but, as we shall see momentarily, only of certain equivalence classes of the extended Coulomb phases for the theory after weakly gauging the classical flavor symmetry rotating the hypermultiplets, as it is these that can be related each to a distinct descendant gauge theory. To specify a Coulomb phase we shall use the object known as a box graph that was introduced in [44], and that has been summarized in section 2, and for the equivalence classes that we shall define it is necessary to know such box graphs for the fundamental or vector reprensetations of $\mathrm{U}(N), \operatorname{Sp}(N)$ and $\mathrm{SO}(N)$, which are determined in the aforementioned paper.

The procedure followed in this section to obtain the set of descendant gauge theories is as follows. We will first weakly gauge the classical flavor symmetry that rotates the hypermultiplets associated to a flavor node in a $5 \mathrm{~d}$ gauge theory quiver. This theory has a Coulomb branch, $\mathcal{C}^{\text {w.g. }}$, and in the limit where we take the gauge coupling of the weakly gauged flavor symmetry to zero, this Coulomb branch fractionates. The result is a set of Coulomb branches of all of the descendant gauge theories arising as mass deformations of the original gauge theory. There may be redundancies in this description as, for instance, the same Coulomb branch for a descendant can appear multiple times within this set. It is vital, therefore, to, after determining in a redundant way all of the descendant theories, identify those identical Coulomb branches as belonging to the same theory. We refer to these identifications as "discrete dualities", although this is something of a misnomer since they are often not dualities but directly equivalent descriptions of the same theory. In this section we will determine the larger set of descendants, where there are still these redundencies. This will first be carried out for the single node gauge theories, as the logic therein will extend multiplicatively across arbitrary quivers.

The key concepts that we will define and determine for all gauge theories, are equivalence classes of box graphs called a flavor-equivalence class, and associated graphs, the Box Graph Combined Fiber Diagram (BG-CFD), as a collection of vertices and edges; these definitions appear in section 3.1. In sections 3.4, 3.5, and 3.6 we will consider all of the gauge theoretic descendants for single gauge node quivers of the form

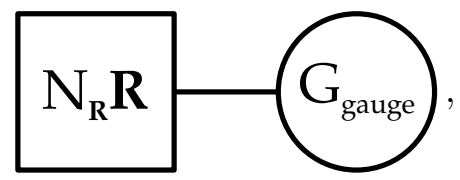


where $G_{\text {gauge }}$ is a simple Lie group and the representation $\boldsymbol{R}$ is, respectively, complex, quaternionic, or real. Each of these descendants will be in a one-to-one correspondence to a flavor-equivalence class, and will have an associated BG-CFD. In section 3.7 we show how this analysis extends simply to determine all of the descendant gauge theories associated to arbitrary quiver gauge theories with building blocks (3.1), by gluing together such gauge nodes with bifundamental hypermultiplets, by attaching multiple flavor nodes to a given gauge node, or combinations of both of these constructions.

\subsection{Flavor-equivalence classes and box graph CFDs}

In section 2.4 we discussed the gauge theory phases for the $\mathrm{SU}(2)$ gauge theory with a weakly gauged $\mathrm{SO}(16)$ flavor symmetry and matter transforming in the $(\mathbf{2}, \mathbf{1 6})$ representation. The set and structure of the Coulomb phases was in a one-to-one correspondence with the set of $\mathrm{SU}(2)$ gauge theories that arise from mass deformations of $\mathrm{SU}(2)+8 \boldsymbol{F}$. For more general gauge theories this will not always be the case, indeed the Coulomb phases after weakly gauging the classical flavor symmetry rotating the hypermultiplets will be in a many-to-one map onto the mass deformations of the original gauge theory. The reason for this is that there will be distinct phases in the weakly gauged theory where the distinction is only moving amongst the Coulomb phases of the original gauge theory; we are not interested in the distinction between these phases as they do not correspond to mass deformations of the original theory, but instead to moving on its Coulomb branch. To remove this redundancy and to restore a one-to-one relationship, we define an appropriate equivalence class.

Definition 3.1 Flavor-equivalence class of box graphs. Consider two box graphs associated to Coulomb phases of a gauge theory with symmetry groups $G_{\text {gauge }} \times G_{\mathrm{BG}}$, where the $G_{\mathrm{BG}}$ is considered as a weakly gauged flavor symmetry. Denote the simple roots of $G_{\text {gauge }}$ by $\alpha_{i}^{\text {gauge }}$ and those of $G_{\mathrm{BG}}$ by $\alpha_{j}^{\mathrm{BG}}$. Then, these two box graphs are flavor-equivalent if the splitting of the $\alpha_{j}^{\mathrm{BG}}$ contains, in total, the same subset of roots $\alpha_{i}^{\text {gauge }}$ of $G_{\text {gauge }}$. Furthermore, if none of the $\alpha_{j}^{\mathrm{BG}}$ split in the box graphs then the two box graphs are flavor-equivalent if and only if they are identical.

It is easy to see that, for an irreducible representation $\left(\boldsymbol{R}_{\text {gauge }}, \boldsymbol{R}_{\mathrm{BG}}\right)$ of $G_{\text {gauge }} \times G_{\mathrm{BG}}$, the flavor-equivalence class is completely determined by the decoration of the weights of $\boldsymbol{R}_{\mathrm{BG}}$ associated to the highest and lowest weights of $\boldsymbol{R}_{\text {gauge }}$, as they appear in the tensor product of the weights that form the product representation $\left(\boldsymbol{R}_{\text {gauge }}, \boldsymbol{R}_{\mathrm{BG}}\right)$. Furthermore, when there are multiple different matter representation of $G_{\text {gauge }}$ there is a different flavor symmetry associated to each matter field, and so the flavor-equivalence classes are multiplicative across the different matter fields; a prominent example of this will appear in section 3.7.

To determine the structure of the tree of mass deformations we need in addition the following definitions.

Definition 3.2 Flop transitions for flavor-equivalence classes. Two flavor-equivalence classes of box graphs are related by a flop transition if there exist representatives of each equivalence class which are related by a flop transition. 
Definition 3.3 F-extremal weights. A weight is F-extremal, if it is an extremal weight which, when flopped, changes the flavor-equivalence class.

Definition 3.4 F-extremal weights inside the combined roots of the gauge group. The flavor-equivalence class is associated to the splitting

$$
\sum_{j} \alpha_{j}^{\text {gauge }} \rightarrow \sum_{\ell} \alpha_{\ell}^{\mathrm{BG}}+\sum_{m} \epsilon_{m} L_{m}^{\epsilon_{m}}
$$

where $L_{m}^{\epsilon}$, with $\epsilon= \pm$ are, by definition, a subset of the F-extremal weights of this flavorequivalence class. We refer to the $L_{m}^{\epsilon_{m}}$ that appear in (3.2) as F-extremal weights inside the combined roots of the gauge group.

Moreover, this splitting associated with a reduced (flavor-equivalent) box graph defines the box graph CFD (BG-CFD). This is a sub-graph of the full CFD [1] that is associated to the UV fixed point of the flavor-equivalence class.

Definition 3.5 Box graph $C F D(B G-C F D)$. Given a flavor-equivalence class the $B G$ - $C F D$ is the intersection graph of the F-extremal weights inside the combined roots of the gauge group and the flavor roots $\alpha_{\ell}^{\mathrm{BG}}$ that appear in (3.2).

An example of a BG-CFD is shown in figure 5. The BG-CFD encodes the part of the flavor symmetry of an SCFT that is manifest in the weakly-coupled gauge theory description. It forms generically a strict subgraph of the CFD associated to said SCFT. A complete set of them is listed in table 2 .

Given these definitions in the remainder of this section we are going to determine the flop graph of all flavor-equivalence classes of box graphs for an arbitrary gauge theory quiver built out of nodes corresponding to the simple gauge theories listed in table 1 . Furthermore we will determine the (disconnected) graphs, the BG-CFDs, associated to each quiver gauge theory. We proceed by first determining the flavor-equivalence classes, and the BG-CFDs, for the single node quivers listed in the aforementioned table.

We summarize the results of this section, the number of flavor-equivalence classes for the gauge theories with a simple gauge algebra as listed in table 1, and arbitrary quivers built therefrom. Similarly, for each possible classical flavor group, we summarize the BGCFDs in table 2.

\subsection{An example: $\mathrm{SU}(3)+9 F$}

To fill these definitions with some life, before studying the single gauge node quivers comprehensively, we first work through an example in some detail. Consider the rank two theory $\mathrm{SU}(3)+9 \boldsymbol{F}$. The classical flavor symmetry is $\mathrm{U}(9)$ and we consider box graphs for the $(\mathbf{3}, \mathbf{9})$ of $\mathrm{SU}(3)_{\text {gauge }} \times \mathrm{U}(9)_{\mathrm{BG}}$. The representation graph is shown in figure 3 . The complete set of flavor-equivalence classes for this theory are shown in figure 30 . To understand 


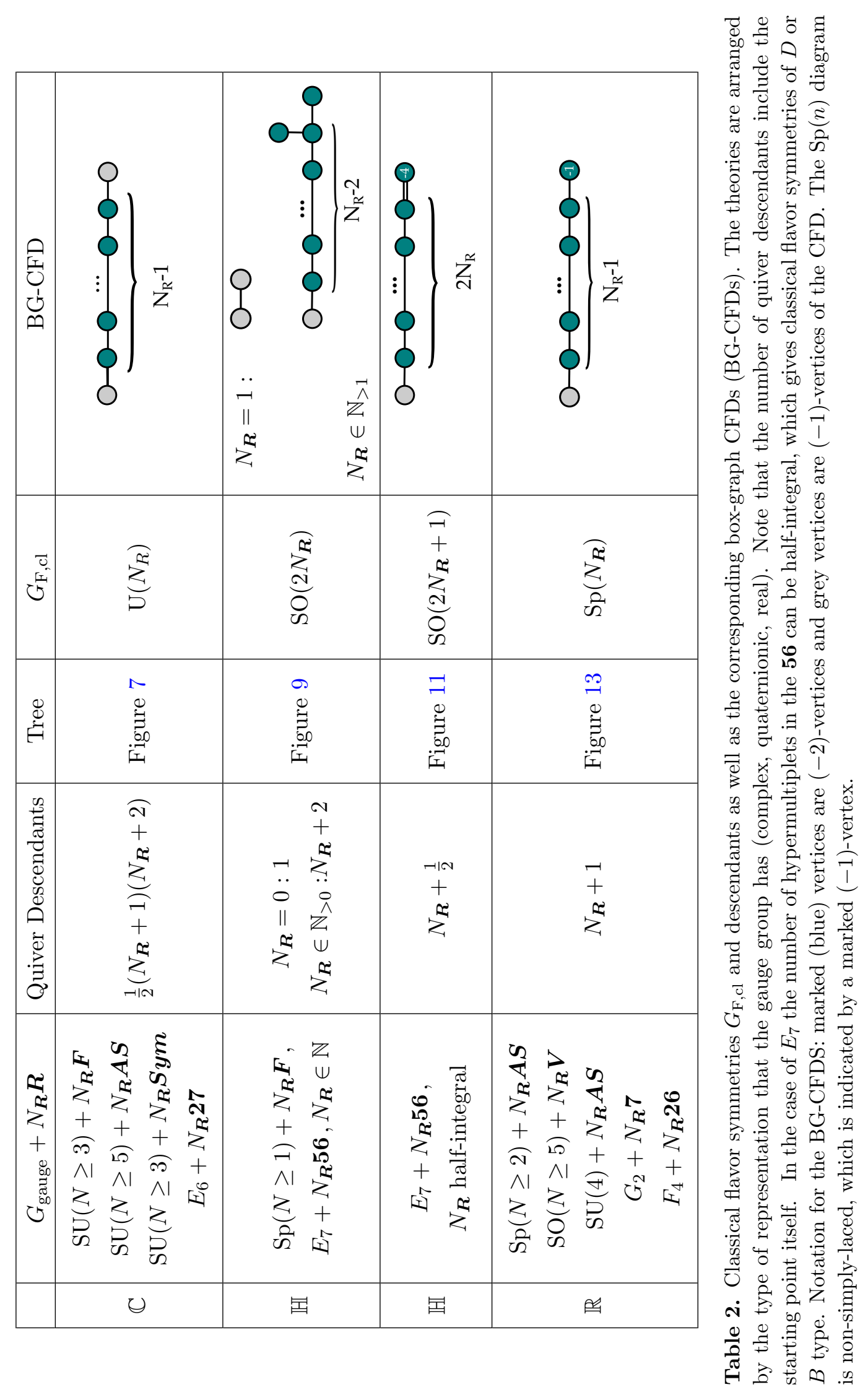




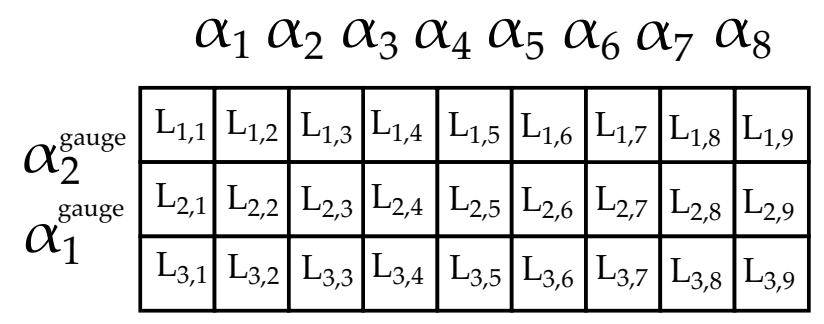

Figure 3. Representation graph for $(\mathbf{3}, \mathbf{9})$ of $\mathrm{SU}(3)_{\text {gauge }} \times \mathrm{U}(9)_{\mathrm{BG}} \cdot L_{i, j}=L_{i}^{\mathrm{SU}(3)}+L_{j}^{\mathrm{SU}(9)}$, where $L_{k}$ indicates the fundamental weights of the respective groups. $\alpha_{i}$ are the simple roots of the classical flavor symmetry $\mathrm{U}(9)$, and $\alpha_{i}^{\text {gauge }}$ those of the gauge group $\mathrm{SU}(3)$.

them in more detail, consider the flavor-equivalence class

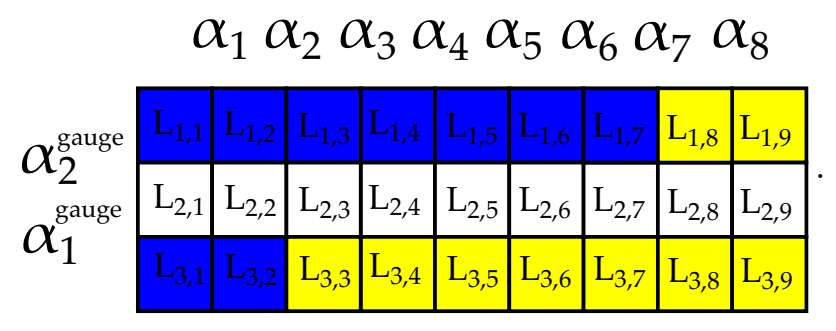

The middle row has no sign assignment, as any consistent sign-assignment, which is subject to the flow rules, given the top and bottom rows, gives a representative of this equivalence class. It follows, e.g., immediately from the flow rules that the sign assignment for $L_{2,1}$ and $L_{2,2}$ has to be + (blue), whereas $L_{2,8}$ and $L_{2,9}$ have - (yellow). The complete set of flavor-equivalent box graphs associated to this equivalence class are shown in figure 4 .

These are all flavor-equivalent in the sense that the splitting of the sum of roots of the gauge group contain the same roots of the flavor symmetry - in this case $\alpha_{3}, \alpha_{4}, \alpha_{5}, \alpha_{6}$. Note that $\alpha_{2}$ and $\alpha_{7}$ split, into the sum of weights $L_{3,2}+\left(-L_{3,3}\right)$ and $L_{1,7}+\left(-L_{1,8}\right)$; these are F-extremal weights, cf. definition 3.3. In each representative of the flavor-equivalence class, the splitting of $\alpha_{i}^{\text {gauge }}$ is different, however the sum of them always contain the same set of flavor roots. That is, the splitting (3.2) for this flavor-equivalence class is

$$
\alpha_{1}^{\text {gauge }}+\alpha_{2}^{\text {gauge }} \rightarrow \alpha_{3}+\alpha_{4}+\alpha_{5}+\alpha_{6}+\left(-L_{3,3}\right)+L_{1,7}
$$

Note that $-L_{3,3}$ and $L_{1,7}$ are the F-extremal weights inside the combined gauge roots, cf. definition 3.4 As one can check using $\alpha_{i}=L_{i}-L_{i+1}$, this is indeed true, and follows directly from the sign assignments indicated in the box graph. The different representatives in the flavor-equivalence class differ by those precisely the splitting is distributed among $\alpha_{1}^{\text {gauge }}$ and $\alpha_{2}^{\text {gauge }}$.

Finally, the BG-CFD (see definition 3.5) is given by the chain of -2 vertices corresponding to the roots $\alpha_{3}, \alpha_{4}, \alpha_{5}, \alpha_{6}$, as well as the two F-extremal weights, which are -1 vertices. This is shown in figure 5 . 

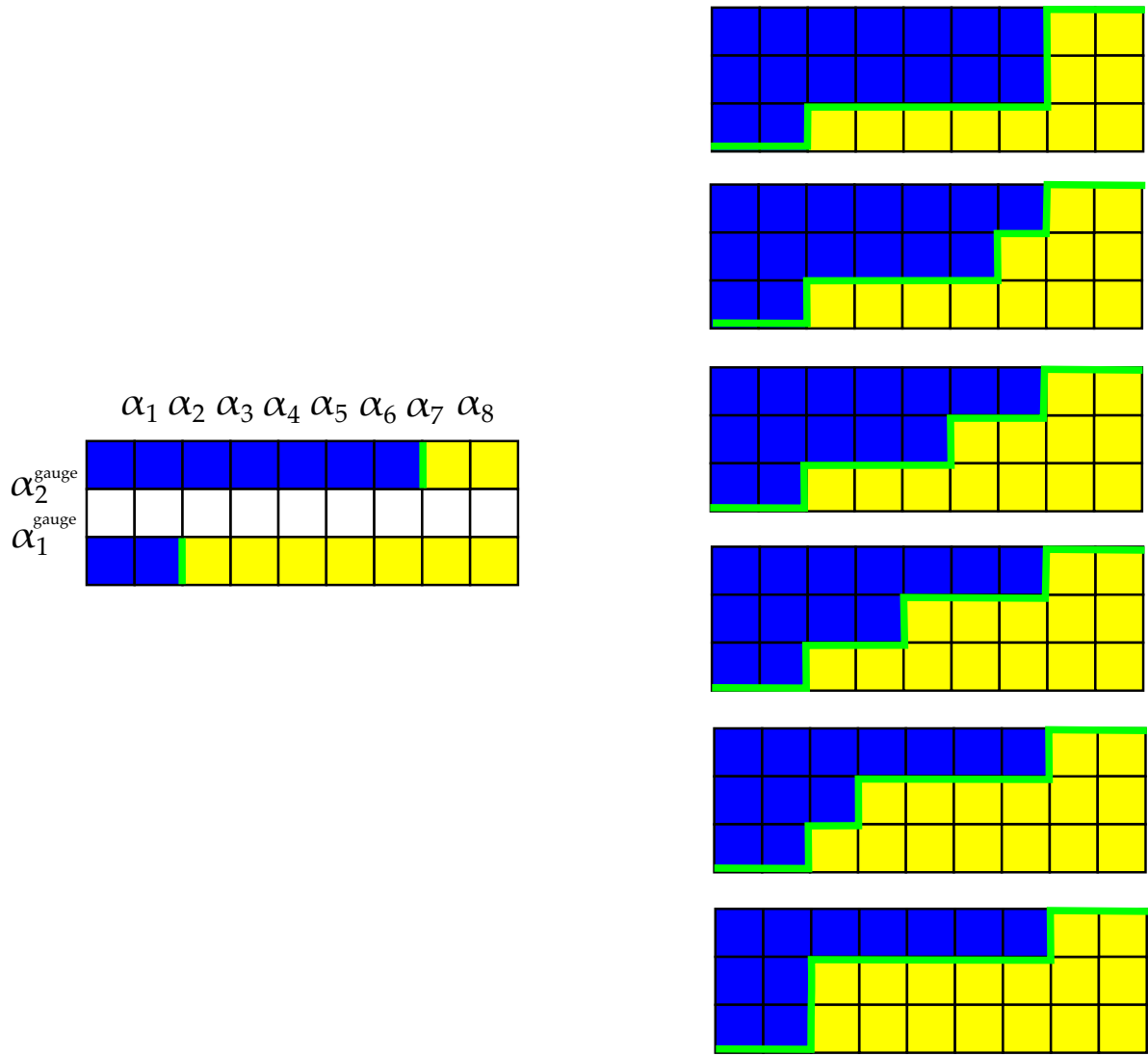

Figure 4. An example of a flavor-equivalence class for $\mathrm{SU}(3)+9 \boldsymbol{F}$. The equivalence class is shown on the left-hand side - there we indicate in green the key characteristics of the associated box graph: the splitting of both roots of the gauge group $\alpha_{i}^{\text {gauge }}, i=1,2$ combined will contain all the roots $\alpha_{3}, \alpha_{4}, \alpha_{5}, \alpha_{6}$. On the right-hand side, we show the complete set of box graphs that comprise the flavor-equivalence class. E.g., the top diagram corresponds to the case where the splitting of $\alpha_{1}^{\text {gauge }}$ contains all $\alpha_{i}, i=3,4,5,6$. In the second one, which is related by a flop to the top one, $\alpha_{1}^{\text {gauge }}$ does not contain $\alpha_{6}$, which is now part of the splitting of $\alpha_{2}^{\text {gauge }}$, etc.

\subsection{From box graphs to 5d gauge theories and SCFTs}

We will now make the connection between box graphs, which capture the Coulomb branch phases of $5 \mathrm{~d}$ gauge theories, and the flavor-equivalence classes of these box graphs, to fivedimensional superconformal field theories. So far, we characterized the Coulomb branch phases of a $5 \mathrm{~d} G_{\text {gauge }}$ theory, whose classical flavor symmetry $G_{\mathrm{BG}}$ has been weakly gauged. The key idea to relate these concepts to 5 d gauge theories with gauge symmetry $G_{\text {gauge }}$ and their SCFT limits is to identify the $G_{\mathrm{BG}}$ with the classical flavor symmetry of the marginal $G_{\text {gauge }}$ theory.

To obtain a theory that has a UV fixed point in $5 \mathrm{~d}$, we need to decouple hypermultiplets from the marginal theory. That is, we add mass terms to matter charged under $G_{\text {gauge }}$ and formally send the mass to infinity. In terms of the extended Coulomb branch, where we treat the classical flavor symmetry $G_{\mathrm{BG}}$ as a weakly gauged symmetry, this is achieved by passing to a new flavor-equivalence class - i.e., performing a flop transition on the box graph. 


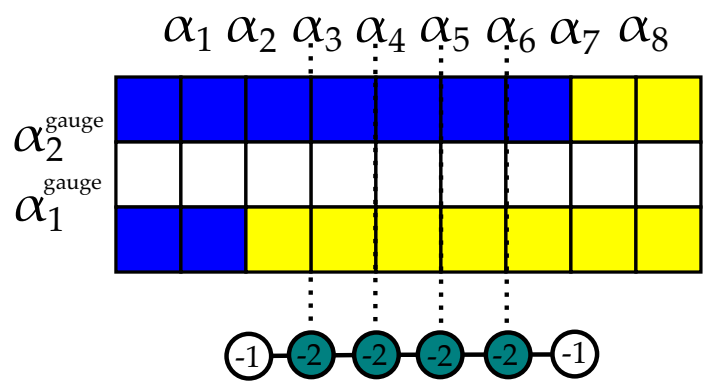

Figure 5. Flavor-equivalence class and BG-CFD: in this figure we show an example for a flavorequivalence class of Coulomb branch phases for $\mathrm{SU}(3)$ with $9 \boldsymbol{F}$. The top graph is the box graph reduced to its flavor-equivalence class by omitting the sign assignments in the middle row: all sign assignments consistent with the standard box graph rules would correspond to the same combined gauge root splitting. This is encoded in the CFD-subgraph that we refer to as BG-CFD: the roots $\alpha_{i}, i=3,4,5,6$ of $G_{\mathrm{BG}}=\mathrm{U}(9)$ that participate in the splitting of the roots $\alpha_{i}^{\text {gauge }}$ of $G_{\text {gauge }}=\mathrm{SU}(3)$ are $(-2)$-vertices in the CFD - shown at the bottom. The $(-1)$-vertices of the CFD correspond to the F-extremal weights $\left(-L_{3,3}\right)$ and $L_{1,7}$.

More precisely, we can rephrase the key property of the marginal theory as follows. Points in the extended Coulomb branch where $G_{\text {gauge }}$ is unbroken correspond to the marginal theory, where the manifest flavor symmetry is $G_{\mathrm{BG}}$, when the group $G_{\mathrm{BG}}$ is also unbroken. In terms of the Coulomb branch parameters $\phi$, gauge enhancement to $G_{\text {gauge }}$ occurs when ${ }^{6}\left\langle\phi, \alpha_{i}^{\text {gauge }}\right\rangle=0$ for all gauge roots. Therefore, the subset of the extended Coulomb branch (with weakly gauged $G_{\mathrm{BG}}$ ) describing the marginal theory in the above sense is one where we have

$$
\forall i:\left\langle\phi, \alpha_{i}^{\text {gauge }}\right\rangle=0 \quad \text { and } \quad \forall j:\left\langle\phi, \alpha_{j}^{\mathrm{BG}}\right\rangle=0
$$

which is nothing other than the point in the extended Coulomb branch

$$
\phi=0 .
$$

In terms of the box graphs, this condition applies exactly when the combined splitting of the gauge roots (3.2) contain all roots of $G_{\mathrm{BG}}$. In this case, $\left\langle\phi, \alpha_{i}^{\text {gauge }}\right\rangle=0$ implies $\left\langle\phi, \alpha_{j}^{\mathrm{BG}}\right\rangle=\left\langle\phi, L_{m}\right\rangle=0$. (Note that the signs in (3.2) are precisely such that $\left\langle\phi, \epsilon_{m} L_{m}^{\epsilon_{m}}\right\rangle \geq 0$.) This means that also linear combinations $\lambda$ out of $L_{m}$ and $\alpha_{j}^{\mathrm{BG}}$, which fill out representations of $G_{\mathrm{BG}}[44,49-51]$, have $\langle\phi, \lambda\rangle=0$. Physically, this characterizes the Coulomb phases of the marginal theory as those where, when the gauge symmetry $G_{\text {gauge }}$ is restored, the massless charged matter transforms under the full $G_{\mathrm{BG}}$ - which by definition was the classical flavor symmetry of the marginal $G_{\text {gauge }}$ theory.

\footnotetext{
${ }^{6}$ Note that when we write an expression like $\left\langle\phi, \alpha_{j}\right\rangle$ we are silently extending the root $\alpha_{j}$ of $G$ to the root lattice of the full semi-simple gauge group.
} 
In the example of the last subsection, $\mathrm{SU}(3)+9 \boldsymbol{F}$, the marginal theory is thus characterized by flavor-equivalence class represented by the box graph

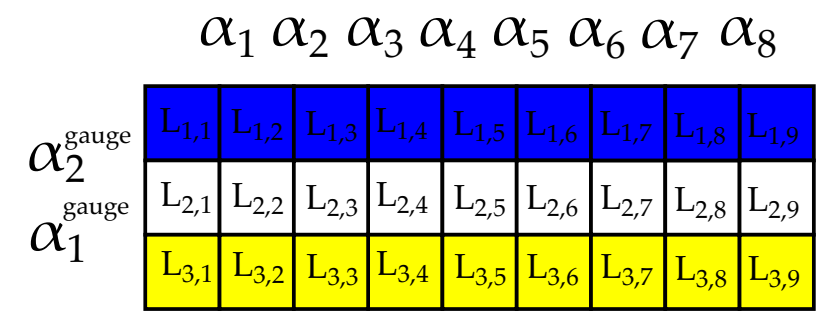

where the representatives of the equivalence class have any sign assignment that is consistent with the flow rules (2.20). Indeed, the box graph rules stipulate that all roots $\alpha_{i}$ of the $\mathrm{SU}(9) \subset \mathrm{U}(9)_{\mathrm{BG}}$ appear in the splitting (3.2) of $\alpha_{1}^{\text {gauge }}+\alpha_{2}^{\text {gauge }}$.

A mass deformation that decouples a hypermultiplet requires a mass term that remains non-zero (and can be sent to $\pm \infty$ ) when $\left\langle\phi, \alpha_{i}^{\text {gauge }}\right\rangle=0$. This results in a smaller flavor symmetry $G_{\mathrm{F}, \mathrm{cl}} \subset G_{\mathrm{BG}}$, whose rank is lowered by one compared to $G_{\mathrm{BG}}$. In the context of having weakly gauged $G_{\mathrm{BG}}$, this must therefore correspond to a phase on the extended Coulomb branch, where there is one flavor root $\alpha_{j}^{\mathrm{BG}}$ with $\left\langle\phi, \alpha_{j}^{\mathrm{BG}}\right\rangle \neq 0$ even when $\left\langle\phi, \alpha_{i}^{\text {gauge }}\right\rangle=0$. The associated box graph of such a phase thus implies a combined splitting of the gauge roots which leaves out one flavor root, whose mass may be identified with the non-zero Coulomb branch parameter.

Starting from the flavor-equivalence class of the marginal theory, such a phase is obtained from a flop, i.e., a change of sign assignment of an F-extremal weight. After the flop, the resulting $G_{\text {gauge }}$ theory has less matter, and correspondingly a smaller classical flavor group $G_{\mathrm{F}, \mathrm{cl}} \subset G_{\mathrm{BG}}$, specified by the roots $\alpha_{j}^{\mathrm{BG}}$ which are still contained in the splitting of the gauge roots.

Returning to our SU(3) example, we recognize $L_{1,9}$ and $-L_{3,1}$ to be the two F-extremal weights in the box graph (3.7). For concreteness consider changing the sign assignment of $L_{1,9}$. In the field theory picture, without the gauging of the flavor symmetry, this corresponds to the decoupling of a hypermultiplet associated with this weight, under the flavor symmetry group. After the flop the flavor-equivalence class is

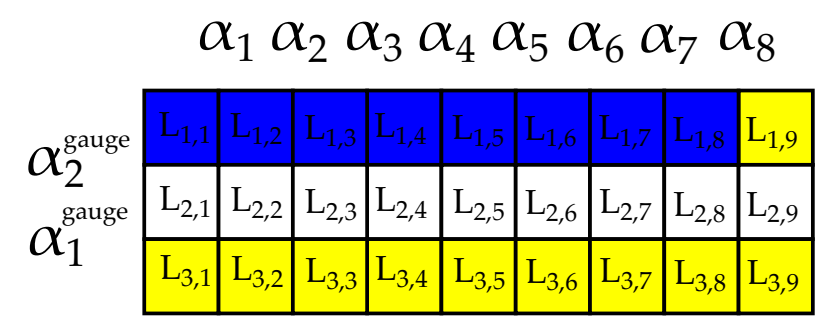

The flow rules imply that the sign assignment for $L_{2,9}$ is - as well, and in the splitting of $\alpha_{1}^{\text {gauge }}+\alpha_{2}^{\text {gauge }}$, only the roots $\alpha_{1}, \cdots, \alpha_{8}$ appear. This means that we have decoupled one fundamental flavor and ended up with an $\mathrm{SU}(3)+8 \boldsymbol{F}$ theory. Consistently, the flavor symmetry of this descendant gauge theory is $G_{\mathrm{F}, \mathrm{cl}}=\mathrm{U}(8)$, whose roots are precisely $\alpha_{1}, \cdots, \alpha_{7}$. If on the other hand we flop $-L_{3,1}$, then $L_{2,1}$ has fixed sign assignment + by the flow rules, and the flavor roots $\alpha_{i}$ that appear in the splitting of the gauge roots are 
$\alpha_{2}, \cdots, \alpha_{8}$. Continuing in this fashion, we can eventually reach the phase (3.3) described earlier, after having flopped $L_{1,9}, L_{1,8},-L_{3,1}$ and $-L_{3,2}$.

Note that after flopping either $L_{1,9}$ or $-L_{3,1}$, both phases define a $\mathrm{SU}(3)+8 \boldsymbol{F}$ theory with classical $G_{\mathrm{F}, \mathrm{cl}}=\mathrm{U}(8)$ flavor symmetry. On the other hand, the different embedding of it into the original $\mathrm{U}(9)_{\mathrm{BG}}$ flavor group will lead to a different superconformal flavor enhancement as we approach the SCFT limits of these two descendant gauge theories. In fact, in case of $\mathrm{SU}(3)$ (or any $\mathrm{SU}(n)$ ) gauge theories in $5 \mathrm{~d}$, the box graphs must be supplemented by the discrete Chern-Simons level $k$, which we have neglected so far. However, it is crucial that $|k|=\frac{3}{2}$ for $\mathrm{SU}(3)+9 \boldsymbol{F}$ to be a marginal theory [9]. The two different descendants, flopping either on $L_{1,9}$ or $-L_{3,1}$, then correspond to $\mathrm{SU}(3)+8 \boldsymbol{F}$ with $|k|=1$ or $|k|=2$, respectively. The different superconformal flavor enhancements of these theories cannot be described by the box graphs alone, but requires a little geometric input related to M-theory realizations of $5 \mathrm{~d}$ gauge theories, see sections 7 and 8 . However, a more succinct portrayal of this process can be developed using the embedding of the BG-CFDs into the CFD description of 5d SCFTs developed in [1,2]. This will be one of the main themes of the present work.

In summary, flops (or changes of sign assignments) in the $G_{\text {gauge }} \times G_{\mathrm{BG}}$ flavorequivalence classes of box graphs provides an alternative description of decoupling a matter hypermultiplet of a $G_{\text {gauge }}$ gauge theory. Starting with the weakly coupled marginal description with gauge group $G_{\text {gauge }}$ and matter transforming under the flavor symmetry $G_{\mathrm{BG}}$, successive flop transitions of flavor-equivalence classes map out all descendants with a $G_{\text {gauge }}$ description, while simultaneously keeping track of their classical flavor symmetry $G_{\mathrm{F}, \mathrm{cl}} \subset G_{\mathrm{BG}}$. Each of these gauge theories has a UV fixed point, and thus each flavor-equivalence class corresponds to a 5d SCFT. As noted before, there can be discrete identifications between the flavor-equivalence classes, which then correspond to the same 5d SCFT; this will be discussed later.

\subsection{Complex representations}

In this section we will discuss the gauge theory descendants in terms of flavor-equivalence classes of box graphs for single node quivers of the form (3.1), where the representation $\boldsymbol{R}$ is complex. We will be concerned with the following theories

\begin{tabular}{c|c}
$G_{\text {gauge }}$ & $\boldsymbol{R}$ \\
\hline $\mathrm{SU}(N \geq 3)$ & $\boldsymbol{F}$ \\
$\mathrm{SU}(N \geq 5)$ & $\boldsymbol{A} \boldsymbol{S}$ \\
$\mathrm{SU}(N \geq 3)$ & $\boldsymbol{S y m}$ \\
$E_{6}$ & $\mathbf{2 7}$
\end{tabular}

where $\boldsymbol{F}, \boldsymbol{A S}$, and $\boldsymbol{S y \boldsymbol { m }}$ refer to the fundamental, anti-symmetric, and symmetric representations, respectively. We point out, however, that the analysis herein applies to any such quiver where $\boldsymbol{R}$ is complex, including, for example, the single node gauge theories with exceptional matter that appear for low ranks of the gauge group in [9]. 
In each of the above cases the classical flavor group that rotates the hypermultiplets arising from the flavor node of the quiver is

$$
G_{\mathrm{BG}}=\mathrm{U}\left(N_{\boldsymbol{R}}\right)
$$

After weakly gauging this global symmetry we are determining the structure of the flavorequivalences classes of the box graphs for the theory with gauge group

$$
G_{\text {gauge }} \times \mathrm{U}\left(N_{\boldsymbol{R}}\right)
$$

with matter transforming in the

$$
(\boldsymbol{R}, \boldsymbol{F})
$$

representation, where $\boldsymbol{F}$ is the fundamental representation of $\mathrm{U}\left(N_{\boldsymbol{R}}\right)$. As we have seen in section 3.1, the flavor-equivalence classes are agnostic as to the particular $\boldsymbol{R}$ and $G_{\text {gauge }}$ above, and thus they are completely determined by the classical flavor group which is weakly gauged. Since all complex representations have the same classical flavor group, the only parameter that enters is the number of hypermultiplets on the flavor node, $N_{\boldsymbol{R}}$.

Let us consider the illustrative example where we take $G_{\text {gauge }}=\mathrm{SU}(N)$ and $N_{\boldsymbol{R}} \boldsymbol{R}=$ $N_{f} \boldsymbol{F}$. The flavor-equivalence classes and the tree structure amongst them will be identical for all of the other combinations of gauge groups and matter appearing in (3.9).

After weakly gauging the classical $\mathrm{U}\left(N_{f}\right)$ flavor symmetry rotating the hypermultiplets we are studying the product gauge theory

$$
\mathrm{SU}(N) \times \mathrm{U}\left(N_{f}\right) \quad \text { with } \quad\left(\boldsymbol{N}, \boldsymbol{N}_{\boldsymbol{f}}\right)_{q} \oplus\left(\overline{\boldsymbol{N}}, \overline{\boldsymbol{N}_{\boldsymbol{f}}}\right)_{-q} .
$$

For complex representations, $\boldsymbol{R} \oplus \overline{\boldsymbol{R}}$, it is necessary only to determine the signs associated to the weights of $\boldsymbol{R}$, as this will completely specify the signs associated to the weights of the $\overline{\boldsymbol{R}}$. The positive simple roots for $\mathrm{SU}(N)$ are

$$
\begin{aligned}
\alpha_{1}^{n} & =(2,-1,0,0, \cdots, 0,0,0,0) \\
\alpha_{2}^{n} & =(-1,2,-1,0, \cdots, 0,0,0,0) \\
& \ldots \\
\alpha_{n-2}^{n} & =(0,0,0,0, \cdots, 0,-1,2,-1) \\
\alpha_{n-1}^{n} & =(0,0,0,0, \cdots, 0,0,-1,2),
\end{aligned}
$$

and similarly for $\mathrm{SU}\left(N_{f}\right)$, the semi-simple part of the $\mathrm{U}\left(N_{f}\right)$. The highest weight of the $\left(\boldsymbol{N}, \boldsymbol{N}_{\boldsymbol{f}}\right)$ representation is given by

$$
L_{1,1}=(1,0, \cdots, 0 ; 1,0, \cdots, 0)
$$

where the semi-colon in the middle denotes the join between the highest weight of the fundamental representation of each of the $\mathrm{SU}(N)$ and $\mathrm{SU}\left(N_{f}\right)$ factors. The representation graph for this representation is represented in figure 6 . This is the weight diagram for the $\left(\boldsymbol{N}, \boldsymbol{N}_{\boldsymbol{f}}\right)$ representation displayed as a box graph. 


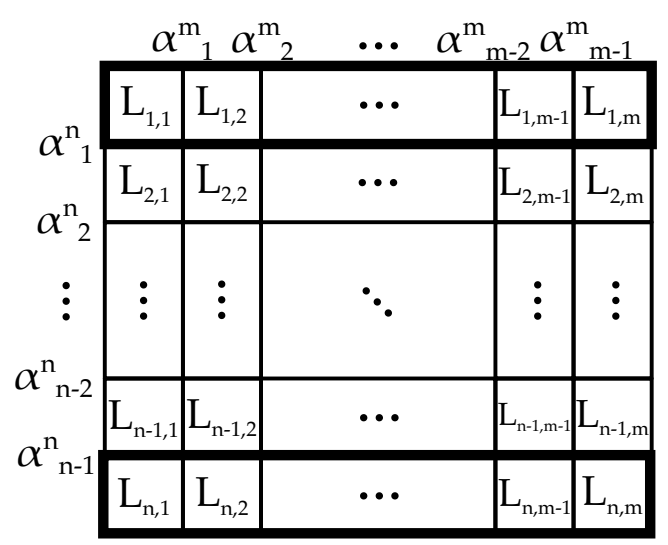

(a)

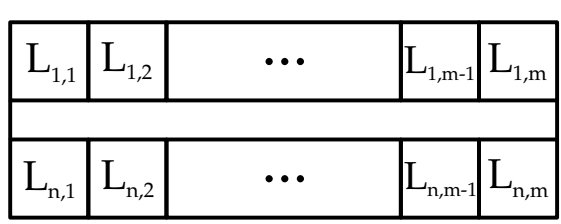

(b)

Figure 6. In (a) is the representation graph, or weight diagram, for $\mathrm{SU}(N)_{k} \times \mathrm{U}\left(N_{f}\right)$ with $\left(\boldsymbol{N}, \boldsymbol{N}_{\boldsymbol{f}}\right)_{q} \oplus\left(\overline{\boldsymbol{N}}, \overline{\boldsymbol{N}_{\boldsymbol{f}}}\right)_{-q}$. Notice that we simply write the $\left(\boldsymbol{N}, \boldsymbol{N}_{\boldsymbol{f}}\right)_{q}$ half of the representation, as the decoration thereon will imply the decoration on the conjugate half. The Coulomb phases are given by all decorations of this box graph subject to the flow rules. In (b) we give the subgraph (marked in boldface in (a)) of the box graph that contains only the weights whose signs are required to be specified to determine the flavor-equivalence class. Any box graphs with the same coloring for the weights in (b) are flavor-equivalent.

The phases are determined by all decorations of the box graph in figure 6 with signs subject to the flow rules (2.20). Equivalently each phase can be characterized by a monotonic path between the lower left and the upper right corners on the $N_{f} \times N$ grid that the representation graph defines. An elementary computation reveals that the total number of such phases is

$$
\left(\begin{array}{c}
N+N_{f} \\
N
\end{array}\right)
$$

Of course, the determination of the total number of gauge theory phases of this weakly gauged product gauge theory is not the goal of this section. This quantity will vary depending on the type of matter representation on the flavor node; it will not just depend on $N_{\boldsymbol{R}}$ in the same manner for all complex representations.

We now turn to the sorting of these phases into flavor-equivalence classes of box graphs. It is clear from figure 6 that, regardless of the coloring in the middle rows, the sum over all of the $\alpha_{\mathrm{BG}}$ can always be written as

$$
\sum_{j=1}^{N-1} \alpha_{j}^{\text {gauge }} \rightarrow L_{1, k_{u}}-L_{N, k_{l}}+\sum_{i=k_{l}}^{k_{u}} \alpha_{i}^{\mathrm{BG}}+\cdots
$$

where the $\cdots$ represents weights that appear in the central $N-3$ rows of the box graph, and where $L_{1, k_{u}}$ is the rightmost box on the upper row decorated with a plus, and $L_{N, k_{l}}$ is the leftmost box on the lower row decorated with a minus. It is clear that the set of $\alpha_{i}^{\mathrm{BG}}$ that are included in the splitting of the $\alpha_{j}^{\text {gauge }}$ depends only on the $k_{u}$ and the $k_{l}$, and therefore the flavor-equivalence class depends only on the choice of consistent decoration 


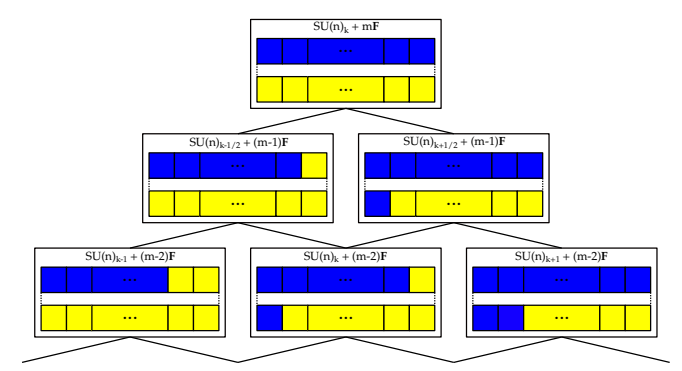

$\cdots$
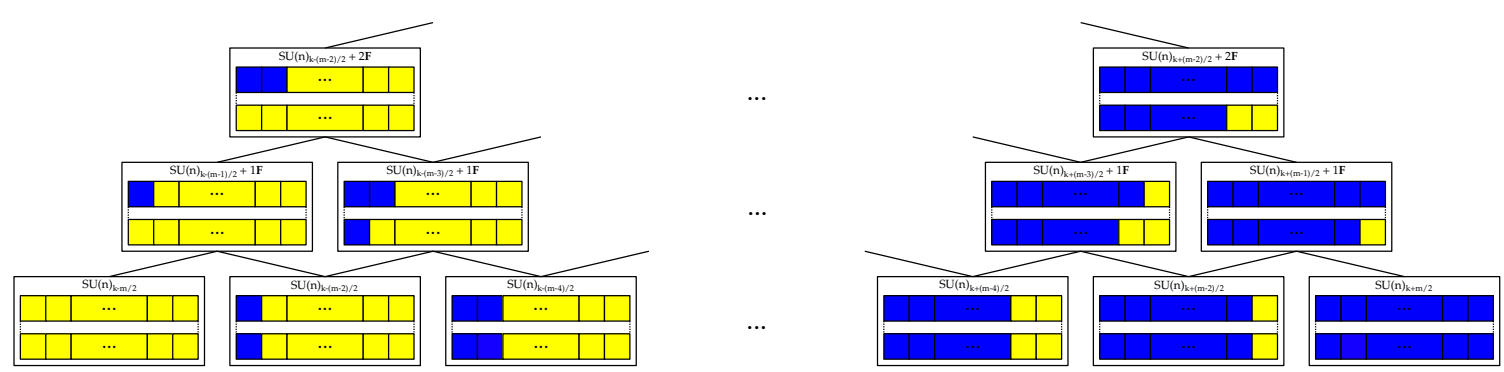

Figure 7. The flop graph for the flavor-equivalence classes of Coulomb phases associated to $\mathrm{SU}(N)$ gauge theory with $\boldsymbol{N}_{\boldsymbol{f}}$ fundmental hypermultiplets. This is identical to the flop graph for the Coulomb branches of all descendant gauge theories with matter in complex representations, as shown in (3.9).

for the uppermost and lowermost rows in the box graph, ${ }^{7}$ corresponding to weights that carry, respectively, the highest and the lowest weight of $\mathrm{SU}(N)$. The flavor-equivalence class is then completely defined by the consistent decoration of the subdiagram of the box graph that is depicted in figure 6(b).

It is straightfoward to see that the total number of consistent decorations that give the flavor-equivalence classes is

$$
\text { \# flavor-equivalence classes for } \mathrm{SU}(N) \text { with } N_{f} \boldsymbol{F}=\frac{1}{2}\left(N_{f}+1\right)\left(N_{f}+2\right) \text {, }
$$

and furthermore one can study the flop transitions between these flavor-equivalence classes to see that they form together in the tree structure that is depicted in figure 7 .

\subsection{Quaternionic representations}

In this section we consider quivers (3.1), where the hypermultiplets transform in a quaternionic, or pseudo-real, representation of the gauge group $G_{\text {gauge }}$. There are only two such kinds of quiver that we need to consider for the purposes of this paper, which are

$$
\begin{array}{c|c}
G_{\text {gauge }} & \boldsymbol{R} \\
\hline \operatorname{Sp}(N \geq 1) & \boldsymbol{F} \\
E_{7} & \mathbf{5 6}
\end{array}
$$

\footnotetext{
${ }^{7}$ Note that even in the case where none of the $\alpha_{j}^{\text {gauge }}$ split, the values of $\left(k_{u}, k_{l}=k_{u}\right)$ specify the flavor-equivalence class, as per the definition.
} 
In this section we shall consider as an example the $\operatorname{Sp}(N)+N_{f} \boldsymbol{F}$, and, as in the case of $\mathrm{SU}(N)$, the analysis shall also apply to $E_{7}+N_{f} \mathbf{5 6}$ because the two representations are quaternionic. In the case of the $\operatorname{Sp}(N)$ there is an anomaly that requires $N_{f}$ to be integer, so to say, that there is an even number of half-hypermultiplets in the fundamental representation of $\operatorname{Sp}(N)$. For $E_{7}$ there is no such anomaly, and $N_{f}$ can be half-integer; since $\mathrm{SO}$ (even) is particularly distinct from $\mathrm{SO}$ (odd) we shall consider the former first, and then move on to the case of $E_{7}$ with an odd number of half-hypermultiplets. In either case the flavor-equivalence class will be specified by the decorated subdiagram of the full box graph that corresponds to the highest and the lowest weights of the representaton of the $\operatorname{Sp}(N)$ or $E_{7}$.

The highest weight of the fundmental representation of $\operatorname{Sp}(N)$ is given by

$$
(1,0, \cdots, 0)
$$

in terms of the usual Cartan-Dynkin labels, and the lowest weight, as the representation is self-conjugate, is given by

$$
(-1,0, \cdots, 0)
$$

To each of these two weights is associated a decoration of the vector representation of the weakly gauged classical flavor group $\mathrm{SO}\left(2 N_{f}\right)$. The vector representation of $\mathrm{SO}\left(2 N_{f}\right)$ has highest weight

$$
(1,0, \cdots, 0)
$$

and is also a self-conjugate representation. Because of this self-conjugacy there is a relation between the weights appearing in the representation $\left(\mathbf{2} \boldsymbol{N}, \mathbf{2} \boldsymbol{N}_{\boldsymbol{f}}\right)$ of $\mathrm{Sp}(N) \times \mathrm{SO}\left(2 N_{f}\right)$, in particular for the weights that appear in the flavor-equivalence class, that is

$$
L_{1, j}=-L_{2 N, 2 N_{f}+1-j} .
$$

In figure 8 we have drawn the subdiagram of the box graph that specifies the flavorequivalence class, and further we have decorated those weights appearing in the flavorequivalence class that cannot be consistently decorated in any other way, and all consistent decorations are given by applying the flow rules (2.20) and (3.23) to this box graph. It is straightforward to see that this yields

$$
N_{f}+2
$$

flavor-equivalence classes when $N_{f}>0$, and when $N_{f}=0$ there is exactly 1 flavorequivalence class. This distinction is a consequence of the fact that when an $\operatorname{Sp}(N)$ gauge theory has no fundamental hypermultipelts it has an additional physical discrete parameter, the $\theta$-angle, which must be specified. The tree structure generated by the flop transitions amongst these flavor-equivalence classes is as given in figure 9 .

In fact, in this case the total number of Coulomb phases is straightforward to determine, and we include the number here for the purposes of later making a comparison between how the number Coulomb phases and the number of flavor-equivalence classes of Coulomb 


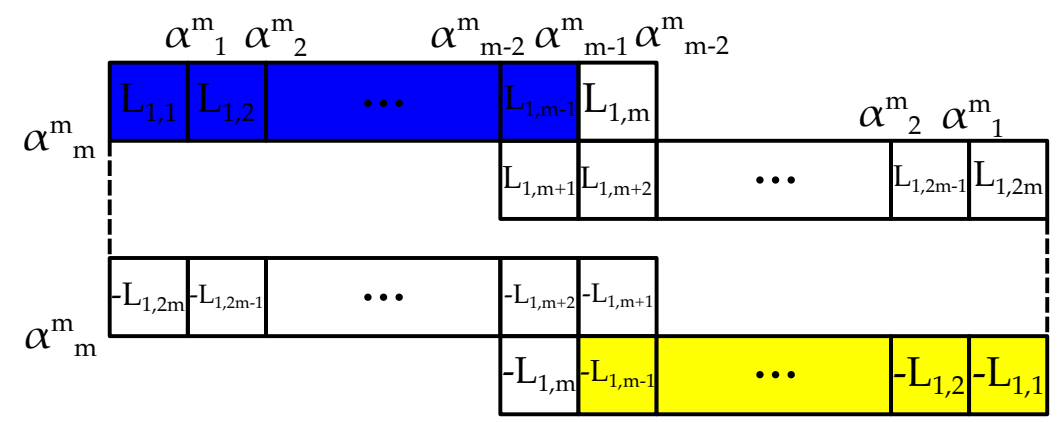

Figure 8. We show the subdiagram of the box graph for which the consistent decoration specifies the flavor-equivalence classes of $\operatorname{Sp}(N)$ with $m=N_{f}$ fundmental hypermultiplets. We have decorated all of the weights whose decoration is fixed by (3.23) and the flow rules (2.20).
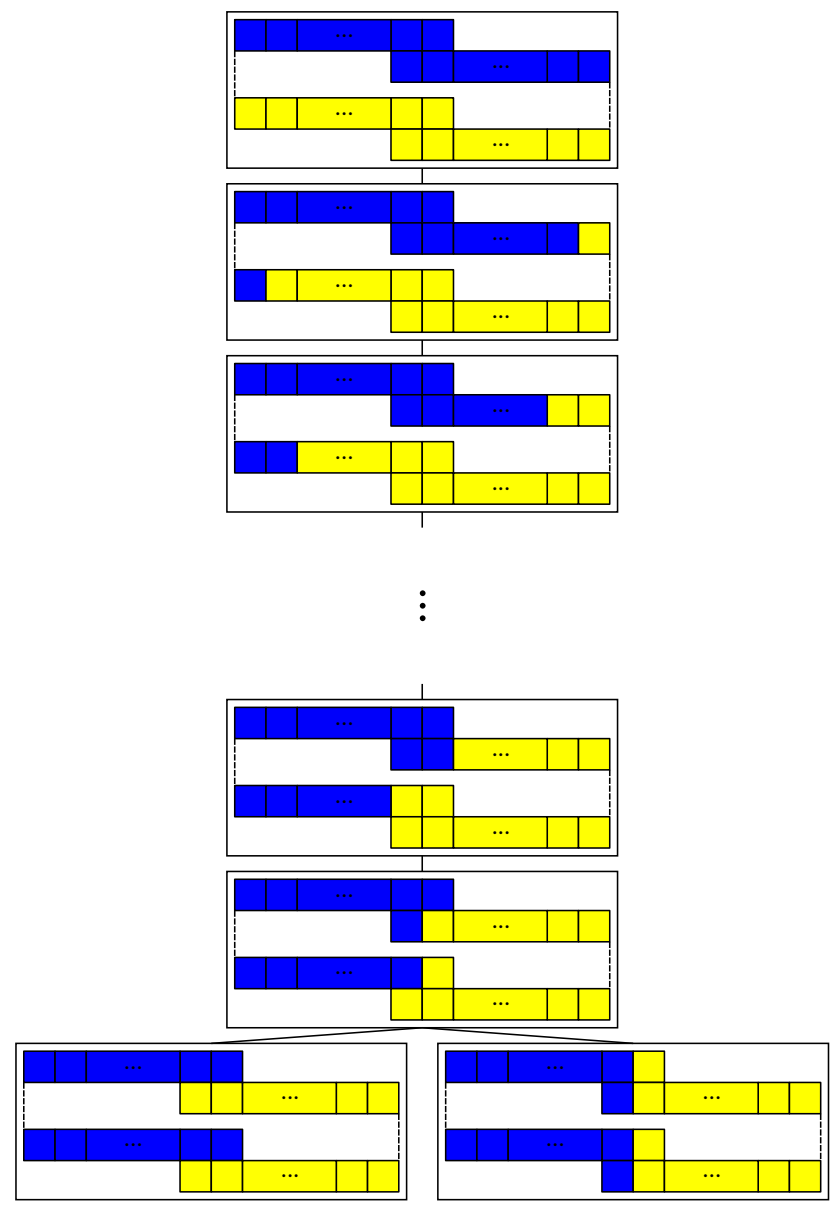

Figure 9. The tree of flop transitions of flavor-equivalence classes, and thus also of the descendant gauge theories, starting from $\operatorname{Sp}(N)+N_{f} \boldsymbol{F}$. The tree structure is identical for the flavor-equivalence classes for $E_{7}+N_{56} \mathbf{5 6}$, as they both have a special orthogonal group as flavor group. 


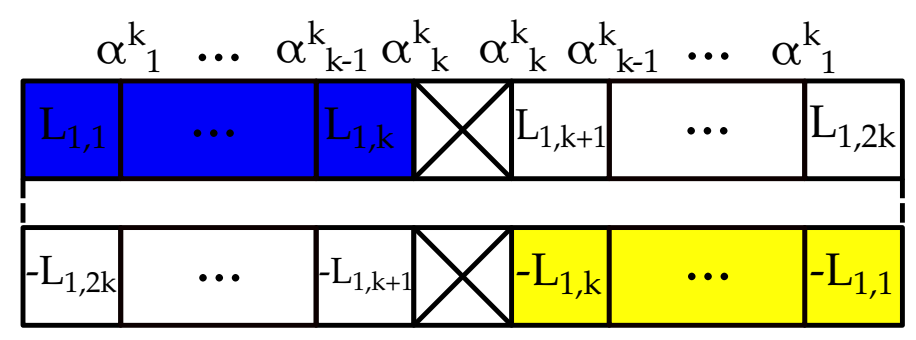

Figure 10. The flavor-equivalence classes associated to $E_{7}$ with $N_{f} \mathbf{5 6}$, where $N_{f}$ is a half-integer, involve the flavor-equivalence classes of $E_{7} \times \mathrm{SO}\left(2 N_{f}\right)$ with matter in the $\left(\mathbf{5 6}, \mathbf{2} \boldsymbol{N}_{\boldsymbol{f}}\right)$. The flavorequivalence classes depend only on the weights written here, and the sign associated to some of them is fixed, as shown. The boxes marked with crosses correspond to the weights that are zero-weights under the $\mathbf{2} \boldsymbol{N}_{\boldsymbol{F}}$ representation, which cannot be consistently assigned a sign.

phases scale when considering quivers that combine such $\operatorname{Sp}(N)$ single gauge nodes. The total number of phases for $\mathrm{Sp}(N) \times \mathrm{SO}\left(2 N_{f}\right)$ with matter in the $\left(\mathbf{2} \boldsymbol{N}, \mathbf{2} \boldsymbol{N}_{\boldsymbol{f}}\right)$ is given by

$$
\left(\begin{array}{c}
N+N_{f}-1 \\
N_{f}-1
\end{array}\right)+\sum_{k=1}^{N} 2\left(\begin{array}{c}
N-k+N_{f}-1 \\
N_{f}-1
\end{array}\right)=\frac{\left(2 N+N_{f}\right) \Gamma\left(N+N_{f}\right)}{\Gamma(N+1) \Gamma\left(N_{f}+1\right)}
$$

where $\Gamma$ is the Euler gamma function.

We now turn to the case where there are an odd number of half-hypermultiplets transforming in a quaternionic representation. This can only occur, in the cases we consider, for $E_{7}$ with matter in the $\mathbf{5 6}$ representation. Since $2 N_{f}$ is odd we can write it as $2 k+1$ and then we are considering the classical flavor group $\mathrm{SO}(2 k+1)$ or $B_{k}$. The Cartan matrix of this algebra is rank $k$ and looks like

$$
\left(\begin{array}{ccccccc}
2 & -1 & 0 & \cdots & 0 & 0 & 0 \\
-1 & 2 & -1 & \cdots & 0 & 0 & 0 \\
0 & -1 & 2 & \cdots & 0 & 0 & 0 \\
& & & \cdots & & & \\
0 & 0 & 0 & \cdots & 2 & -1 & 0 \\
0 & 0 & 0 & \cdots & -1 & 2 & -2 \\
0 & 0 & 0 & \cdots & 0 & -1 & 2
\end{array}\right) .
$$

The highest weights of the fundmental representation of $\mathrm{SO}(2 k+1)$ is

$$
(1,0, \cdots, 0) \text {. }
$$

This representation is depicted, in the usual way, in the undecorated flavor-equivalence box graph that appears in figure 10. Such a representation has the novel feature that it contains a zero-weight, to which a sign cannot be assigned - the weights appearing in the flavor-equivalence class box graph in figure 10 with a cross through them are exactly those such weights that are zero-weights under the weakly gauged $\mathrm{SO}(2 n+1)$ factor.

As previously discussed, the $\mathbf{5 6}$ of $E_{7}$ is a self-conjugate representation and so the weights appearing in the flavor-equivalence class of box graphs are not independent, and 

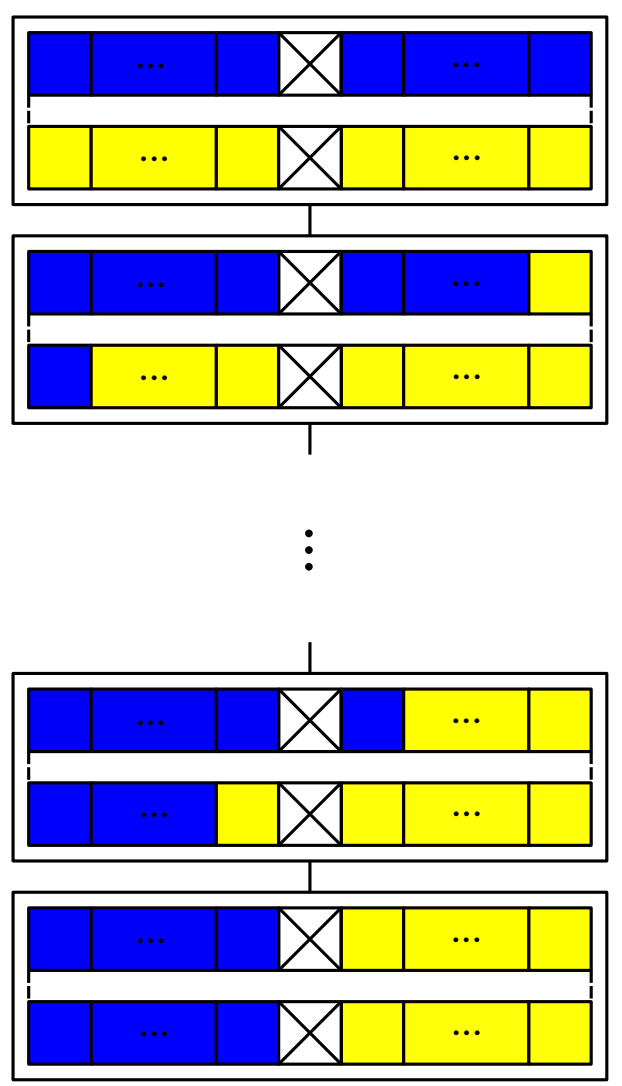

Figure 11. The tree of descendants for $E_{7}$ with $N_{f} \mathbf{5 6}$ where $N_{f}$ is a half-integer. Decoupling one full hypermultiplet descends down the tree.

thus cannot be assigned a sign independently; this interdependence is shown in figure 10, where we also color the boxes for which the sign is fixed a priori, for all phases, by this interdependence together with the flow rules.

The tree of descendants, or flop diagram for the flavor-equivalence classes, is shown in figure 11, and shows that descendants that arise when decoupling one full hypermultiplet of the $\mathbf{5 6}$ at a time. One cannot consistently decouple an odd number of half-hypermultiplets, as there is no possible real mass term, see e.g. [68].

\subsection{Real representations}

In this final case we consider the single gauge node quivers (3.1) where $\boldsymbol{R}$ is in a real representation of $G_{\text {gauge: }}$

\begin{tabular}{c|c}
$G_{\text {gauge }}$ & $\boldsymbol{R}$ \\
\hline $\mathrm{Sp}(N \geq 2)$ & $\boldsymbol{A S}$ \\
$\mathrm{SO}(N \geq 5)$ & $\boldsymbol{V}$ \\
$\mathrm{SU}(4)$ & $\boldsymbol{A} \boldsymbol{S}$ \\
$G_{2}$ & $\mathbf{7}$ \\
$F_{4}$ & $\mathbf{2 6}$
\end{tabular}




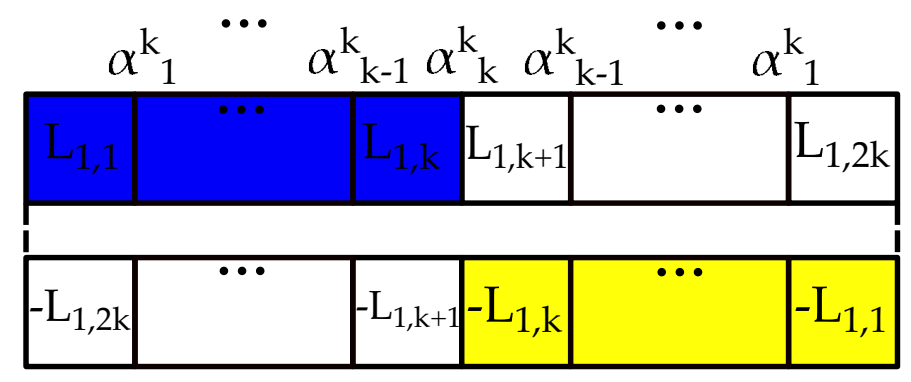

Figure 12. We show the box graph for the flavor-equivalence class of $\operatorname{Sp}(N)$ with $k=N_{a}$ hypermultiplets transforming in the anti-symmetric representation. The boxes whose decoration is fixed by (3.33) are colored. All consistent colorings of the remaining boxes give rise to all consistent flavor-equivalence classes of this theory. Since the structure of the flavor-equivalence box graph is dependent on the $\operatorname{Sp}\left(2 N_{a}\right)$ symmetry rotating the $N_{a}$ hypermultiplets, and not on the particular highest and lowest weights of the anti-symmetric representation of the $\operatorname{Sp}(N)$ but only that they are conjugate, the same structure exists for all theories that have a sympletic symmetry group that acts as rotations on hypermultiplets that transform in a self-conjugate representation of the gauge group. This includes the $\mathrm{SO}(N), G_{2}$, and $F_{4}$ theories of interest in this section.

where we further add that the representaion $\boldsymbol{V}$ is the vector representation. Such theories have a classical flavor group rotating the $N_{\boldsymbol{R}}$ hypermultiplets being

$$
G_{\mathrm{BG}}=\operatorname{Sp}\left(N_{\boldsymbol{R}}\right) .
$$

As such, after weakly gauging this flavor group we are interested in determining the flavorequivalence classes of box graphs for the gauge theory

$$
G_{\text {gauge }} \times \operatorname{Sp}\left(N_{\boldsymbol{R}}\right),
$$

with matter transforming in the representation

$$
(\boldsymbol{R}, \boldsymbol{F}),
$$

where $\boldsymbol{F}$ here denotes the fundamental representation of the $\operatorname{Sp}\left(N_{\boldsymbol{R}}\right)$ rotation group of the hypermultiplets.

The example that we will consider in this section, that will reveal the structure of the flavor-equivalence classes when we have real representations will be $\operatorname{Sp}(N)+N_{a} \boldsymbol{A} \boldsymbol{S}$. These $N_{a}$ hypermultiplets are rotated by an $\operatorname{Sp}\left(N_{a}\right)$ flavor symmetry and thus we are considering the $\left(\boldsymbol{\Lambda}^{\mathbf{2}} \mathbf{2} \boldsymbol{N}, \mathbf{2} \boldsymbol{N}_{\boldsymbol{a}}\right)$ representation of $\operatorname{Sp}(N) \times \operatorname{Sp}\left(N_{a}\right)$. The highest and lowest weights of the $\boldsymbol{\Lambda}^{\mathbf{2}} \mathbf{2} \boldsymbol{N}$ are given by

$$
(0, \pm 1,0, \cdots, 0)
$$

which is again a self-conjugate representation, similarly to the fundamental represention of a symplectic group as has already been discussed. The weight diagram which will capture all of the flavor-equivalence classes for this gauge theory is shown in figure 12 .

Again, because the representation $\left(\boldsymbol{\Lambda}^{\mathbf{2}} \mathbf{2} \boldsymbol{N}, \mathbf{2} \boldsymbol{N}_{\boldsymbol{a}}\right)$ is self-conjugate there is a relationship amongst the weights of the representation. For the weights relevant for the flavorequivalence class this is

$$
L_{1, i}=-L_{2 N+1,2 N_{a}+1-i}
$$



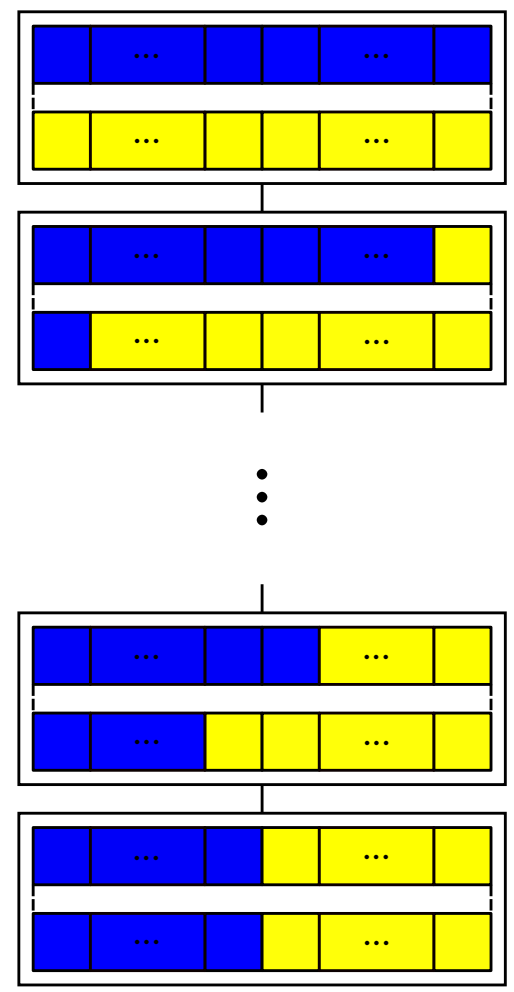

Figure 13. The tree of flavor-equivalence classes for $\operatorname{Sp}(N)+N_{a} \boldsymbol{A} \boldsymbol{S}, \mathrm{SO}(N)+N_{V} \boldsymbol{V}, G_{2}+N_{7} \boldsymbol{7}$, and $F_{4}+N_{26} \mathbf{2 6}$. What is shown are the decorations/colorings of the undecorated part in figure 12 . All of these theories have a symplectic group acting as rotations on the hypermultiplets, this is the key feature that controls the flavor-equivalence classes, and thus each of these theories has the same structure for their flavor-equivalence classes.

Similarly to the case of fundamental matter, there are weights that can only be consistently decorated with one particular sign due to (3.33) combined with the flow rules (2.20). These weights are shown with their necessary decoration in figure 13, and the rest of the flavor-equivalence classes come from the consistent decoration of the remaining undecorated boxes. The total number of flavor-equivalence classes is

$$
N_{a}+1 \text {, }
$$

and furthermore these flavor-equivalence classes arrange themselves, via flop transitions, into the tree shown in figure 13.

To give an explicit example, for the gauge theory $G_{2}+1 \times \mathbf{7}$ the extended Coulomb phases were written down from a geometric realization in [65], and one can see that the four phases found there sort themselves into two flavor-equivalence classes with three and one representatives, respectively.

\subsection{Flavor-equivalence classes for quiver gauge theories}

The quivers that we will consider are those that are built out of gauge nodes that correspond to the gauge theories described in table 1 . There are two ways to build such quivers out of 
the previously analyzed quivers (3.1). Either we chain together gauge nodes of that form, potentially also without any associated flavor node, or else we add more flavor nodes on to a given single gauge node. The number of flavor-equivalence classes is multiplicative across constructing quivers with arbitrary numbers of gauge nodes, out of the single nodes in the table, via gluing two gauge nodes together with bifundamental matter. Such bifundamental matter is uncharged under any of the flavor symmetries, and thus the flavor-equivalence classes, which are defined as those box graphs with the same set of flavor roots contained inside of the gauge roots, are unchanged. So the total number of weakly gauged phases will increase in an intricate way upon gluing, but the flavor-equivalent phases will simply be all ways of choosing one flavor-equivalence classes from the equivalence class associated to each gauge node. The total number of flavor-equivalence classes attached to a quiver, $Q$, is then

$$
n_{Q}=\prod_{G} n_{G},
$$

where $G$ runs over all the gauge nodes in the quiver, and $n_{G}$ is the number of flavorequivalence classes for that gauge node, as given above, and which depends on the flavor nodes attached to that gauge node.

The quantity $n_{G}$ is determined above in the cases where the gauge node has at most one flavor node attached. We will now show that, when multiple flavor nodes are attached, which can only occur for $\mathrm{SU}(N)$ or $\mathrm{Sp}(N)$ gauge nodes if we wish to have a interacting SCFT in the UV limit, the number of flavor-equivalence classes, and thus the number of descendants (counting redundantly) is multiplicative.

We will consider first the set of flavor-equivalence classes from the $5 \mathrm{~d} \mathcal{N}=1$ gauge theory with the following matter fields

$$
\mathrm{SU}(N)_{k} \quad \text { with } \quad N_{f} \boldsymbol{F}+N_{a} \boldsymbol{A} \boldsymbol{S}+N_{s} \boldsymbol{S y \boldsymbol { m }}
$$

where $\boldsymbol{F}, \boldsymbol{A S}$, and $\boldsymbol{S y \boldsymbol { m }}$, respectively, refer to hypermultiplets that transform in the fundamental, anti-symmetric, and symmetric representations of the $\mathrm{SU}(N)$. For the purposes of the flavor-equivalence classes the Chern-Simons level, $k$, will be immaterial, as the box graph is not sensitive to such discrete data. The classical flavor symmetry of this theory is

$$
\mathrm{U}\left(N_{f}\right) \times \mathrm{U}\left(N_{a}\right) \times \mathrm{U}\left(N_{s}\right) .
$$

After weakly gauging the first three factors, which are the perturbative flavor symmetry groups, the theory contains matter that transforms in the

$$
\left(N, N_{f}, \mathbf{1}, \mathbf{1}\right)_{q_{f}} \oplus\left(\Lambda^{2} N, \mathbf{1}, N_{a}, \mathbf{1}\right)_{q_{a}} \oplus\left(\operatorname{Sym}^{2} N, \mathbf{1}, \mathbf{1}, N_{s}\right)_{q_{s}} \oplus \text { c.c },
$$

where the subscripts indicate the charges of the matter fields under the U(1) factor of the $\mathrm{U}\left(N_{\boldsymbol{R}}\right)$ symmetry that rotates the hypermultiplets transforming in the representation $\boldsymbol{R}$. As before, we will assume that $q_{\boldsymbol{R}} \neq 0$ as otherwise this would lead to the decoupling of the $\mathrm{U}(1)$ and thus a changing of the phase structure; this is of course true when the $\mathrm{U}(1)$ is part of a $\mathrm{U}(k)$ global rotation group. It is with respect to these representations that we must determine the flavor-equivalence classes. 
Each of the irreducible representations in (3.38) are charged under different gauge groups, after the weak gauging, and so the subsectors of the Coulomb branch that capture moving the vacuum expectation values of the matter fields of different irreducible representations are orthogonal to each other. As such we can consider the fundamental, anti-symmetric, and symmetric matter under the $\mathrm{SU}(N)$ independently, and the number of flavor-equivalence classes for each of these was determined in section 3.4.

Now we are considering the more general case, where $N_{f}, N_{a}$, and $N_{s}$ are all, in principle, non-zero. As the Coulomb branch has the stucture of the product of the four Coulomb branches give by the Weyl chambers of the $\mathrm{SU}(N)$ and of the three weakly gauged flavor symmetries, and that the vevs under consideration are orthogonal in this space, the total number of flavor-equivalence classes (and indeed the number of Coulomb phases) is simply the product of the total number from each irreducible matter representation. The total number of flavor-equivalence classes is then given by the expression

$$
n_{\mathrm{SU}(N)}=\frac{1}{8}\left(1+N_{a}\right)\left(2+N_{a}\right)\left(1+N_{f}\right)\left(2+N_{f}\right)\left(1+N_{s}\right)\left(2+N_{s}\right) .
$$

Each of these flavor-equivalence classes can be represented by a triplet of consistently decorated diagrams as in figure 6(b), where the length of each is $N_{f}-1, N_{a}-1$, and $N_{s}-1$. This simple structure follows because each of the different kinds of $\mathrm{SU}(N)$ matter all have an $\mathrm{U}\left(N_{\boldsymbol{R}}\right)$ flavor symmetry which rotates the respective hypermultiplets under their fundamental representation. The flop graph of these equivalence classes then has the form of figure 7 , extended into a space spanned by two additional transverse planes, which we do not attempt to draw here.

For $\operatorname{Sp}(N)$ gauge theories one can only have matter transforming in the fundmamental and anti-symmetric representations if one wishes to have a non-trivial interacting fixed point in the UV. We will consider such theories, which we write as

$$
\operatorname{Sp}(N) \quad \text { with } \quad N_{f} \boldsymbol{F}+N_{a} \boldsymbol{A} \boldsymbol{S} .
$$

We note that if $N_{f}=0$ then we must, in addition, specify a discrete $\theta$-parameter for the $\operatorname{Sp}(N)$, being either 0 or $\pi$. The classical flavor symmetry of the theory is

$$
\mathrm{SO}\left(2 N_{f}\right) \times \operatorname{Sp}\left(N_{a}\right)
$$

and when one weakly gauges the first two factors one has matter, which determines the phase structure of the Coulomb branch, transforming in the

$$
\left(N, 2 N_{f}, 1\right) \oplus\left(\Lambda^{2} N, \mathbf{1}, 2 N_{a}\right),
$$

representations of $\operatorname{Sp}(N) \times \mathrm{SO}\left(2 N_{f}\right) \times \mathrm{Sp}\left(N_{a}\right)$. Again, each of these representations can be considered seperately for the purposes of the flavor-equivalence classes and the result follows from sections 3.5 and 3.6. Putting everything together we can determine that the total number of flavor-equivalence classes for $\operatorname{Sp}(N)$ gauge theories with arbitrary $N_{f}$ and $N_{a}$ is

$$
n_{\mathrm{Sp}(N)}=\left\{\begin{array}{ll}
\left(N_{a}+1\right) & \text { if } \quad N_{f}=0 \\
\left(N_{f}+2\right)\left(N_{a}+1\right) & \text { otherwise }
\end{array} .\right.
$$


One example of a multi-node quiver which we will explore more in appendix $\mathrm{A}$ is when the gauge theory is given by the two-gauge-node quiver

$$
M_{1} \boldsymbol{F}-\mathrm{Sp}(1)-\mathrm{Sp}(1)-M_{2} \boldsymbol{F},
$$

where $M_{i} \geq 1$. Such a theory has

$$
\left(M_{1}+2\right)\left(M_{2}+2\right)
$$

flavor-equivalence classes of phases. The total number of gauge theory phases is given by

$$
2\left(M_{1}+2\right)\left(M_{2}+2\right)
$$

where the factor of 2 comes from the two different phases of the bifundamental of the two $\mathrm{Sp}(1)$ factors. In this example we can see that whilst determining the number of Coulomb phases for an arbitrary quiver may be quite involved, the number of flavor-equivalence classes is obtained by a simple combination of the number for each individual gauge node.

\section{Weakly-coupled descriptions from CFDs}

In the previous section we have determined the set of descendants for a given $5 \mathrm{~d} \mathcal{N}=1$ quiver gauge theory. In $[1,2]$ one determined a geometric object, a graph known as a Combined Fiber Diagram, or CFD, that is associated in principle to any 5d or 6d SCFT. Therein it was observed that, if one knows a weakly coupled gauge theory description for a 5d SCFT, then any global symmetry enhancement at the superconformal point, and furthermore the tree of all of the descendants of that SCFT, and thus of the gauge theory, is captured in the CFD.

In this section we will demonstrate that given a CFD the set of weakly coupled quiver gauge theory descriptions that have the associated SCFT at the UV fixed point are heavily constrained. Of particular interest will be to constrain the marginal ${ }^{8} 5 \mathrm{~d}$ quiver gauge theory descriptions of a given 6d SCFTs, as these are conjectured to source all of the $5 \mathrm{~d}$ SCFTs as descendants. One then has to know the "marginal CFD," which is the CFD associated to a $6 \mathrm{~d}$ SCFT, of which many interesting cases are known from $[1,2]$.

Let us briefly recap some of the salient details of $5 \mathrm{~d} \mathcal{N}=1$ quiver gauge theories. A quiver consists of $n_{G}$ gauge nodes, each of which supports some simple non-abelian ${ }^{9}$ gauge group $G_{i}$, such that the total gauge algebra is

$$
G=\prod_{i=1}^{n_{G}} G_{i} .
$$

\footnotetext{
${ }^{8}$ The set of marginal $5 \mathrm{~d}$ gauge theories and the set of $5 \mathrm{~d}$ gauge theories which have a UV fixed point that is a $6 \mathrm{~d}$ SCFT are closely overlapping but distinct sets [9]. In this paper the marginal theories that we consider will have $6 \mathrm{~d}$ fixed points, and thus we will utilize the adjective "marginal" without including the further qualification.

${ }^{9}$ Gauge nodes carrying a U(1) gauge group will not be considered, as quivers with such nodes cannot give rise to an interacting SCFT in the UV [40].
} 
The rank of this gauge algebra will be denoted $r_{G}$. Two gauge nodes can be connected by including matter transforming in the bifundamental ${ }^{10}$ representation of the two gauge algebras. We will assume that any gauge nodes are connected in the most minimal way possible, with the bifundamental matter being either a single hypermultiplet or a single half-hypermultiplet, depending on what is least allowed. Furthermore, we will assume that the quivers under discussion do not have loops. For most of the analysis in this section these two assumptions will be unnecessary, and the analysis is essentially unchanged by relaxing them. In addition a quiver can have matter transforming in a representation $\boldsymbol{R}$ of a single gauge factor; this is the matter captured in the flavor nodes of the quiver.

The global symmetry group of the quiver has three contributions, which can be summarized by writing the rank of the flavor group as

$$
r_{F}=n_{F}+n_{G}+n_{b}
$$

The most obvious contribution is from the number of gauge nodes, $n_{G}$; each simple gauge factor has an associated topological symmetry, $\mathrm{U}(1)_{T}$. The other two factors, $n_{F}$ and $n_{b}$ come from the classical flavor group rotating the charged hypermultiplets of the quiver. These rotation groups depend on the type of representation under which the hypermultiplets transform. They are:

$$
\begin{aligned}
k \text { hypermultiplets in a complex representation } & \Rightarrow \mathrm{U}(k), \\
k \text { hypermultiplets in a real representation } & \Rightarrow \mathrm{Sp}(k), \\
k \text { half-hypermultiplets in a quaternionic representation } & \Rightarrow \mathrm{SO}(k),
\end{aligned}
$$

where the hypermultiplets rotate under the fundamental representation of the global symmetry group. We define $n_{F}$ to be the rank of this combined group for all of the flavor nodes of the quiver. The last quantity, $n_{b}$, is defined to be the rank of flavor group of the bifundamentals connecting the gauge nodes; since $k=1$ for such matter the contribution to $n_{b}$ is zero when the bifundamental is real $\otimes$ quaternionic, and one in all other cases.

The key thrust of this section lies in the fact that the flavor nodes of any marginal quiver description are highly constrained by the structure of the CFD, as the BG-CFD, defined in 3.5, associated with classical flavor of the quiver must be a subgraph of the marginal CFD. Recall that all types of BG-CFDs these are listed in table 2. The reason is that both graphs represent features of the same geometry underlying the M-theory realization. Thus, a necessary condition for a gauge theory to be a consistent effective description of an SCFT is for the BG-CFD of the former to embed into the CFD of the latter.

The geometric details of this relationship will be spelled out in sections 7 and 8 . To get across our main points here, we will review the definition of the CFDs in section 4.1, followed by listing what constraints apply to the prospective quiver from a known CFD in section 4.2; we will find that the possible flavor nodes of any quiver are constrained to be one of a small finite list from the embedding of the BG-CFD inside the CFD, further

\footnotetext{
${ }^{10}$ Adding hypermultiplets charged under different non-trivial representations of the two gauge algebras, or indeed of any number of simple gauge factors, is a priori possible, however we will not consider such quivers here.
} 
usage of the gauge rank and flavor rank, together with the single node constraints of [9] will often allow one to specify the complete quiver more restrictively. We determine the constraints on the possible 5d quiver descriptions for many of the known marginal CFDs and thus for their associated 6d SCFTs.

\subsection{Recap: CFDs}

The CFD $[1,2]$ is a marked undirected graph, where each vertex $C_{i}$ is associated with two integers $\left(n_{i}, g_{i}\right)$ and each edge between the two vertices $C_{i}$ and $C_{j}$ is marked with an integer $m_{i, j}$. In the context of elliptic Calabi-Yau geometries, a CFD can be interpreted as a flop equivalence class among a family of reducible complex surfaces $\mathcal{S}$. Under this interpretation, each vertex $C_{i}$ is a complex curve with self-intersection number $n_{i}$ and genus $g_{i}$, and the integer $m_{i, j}$ is equal to the intersection number $C_{i} \cdot \mathcal{S} C_{j}$.

Qualitatively, the vertices can be classified into the following three classes:

1. The marked vertices, which correspond to flavor curves $F_{i}$, and are usually colored green. Typically, they have labels $\left(n_{i}, g_{i}\right)=(-2,0)$, and are called " $(-2)$-vertices". However, sometimes they are associated with $\left(n_{i}, g_{i}\right)=(-1,0)$ instead, see the $\left(E_{8}, \mathrm{SU}(2)\right)$ CFDs in [2]. The subgraph of such vertices always form the Dynkin diagram of the flavor symmetry of the UV fixed point, $G_{\mathrm{F}}$.

In the presence of some non-simply laced Lie algebra $G$ (such as the $\left(E_{7}, \mathrm{SO}(7)\right)$ case in (5.13)), the flavor curve corresponding to the short root is a collection of $p$ green $(-2)$-vertices that are identical, ${ }^{11}$ where $p$ is the ratio between the length of the long root and the short root of the Lie algebra $G$. Specifically, for $G=B_{k}$, the single short root will be assigned to a reducible vertex, represented by with two $(-2)$-vertices that are encircled. For $G=G_{2}$, the short root will be assigned to a reducible vertex with three $(-2)$-vertices that are encircled. For $G=C_{k}$, there is only a single long root, along with $(k-1)$ short roots. In principle, we need to draw $(k-1)$ reducible vertices which are each containing two $(-2)$-vertices that are encircled, and a single vertex with $\left(n_{k}, g_{k}\right)=(-2,0)$, while these vertices are connected with $m_{i, i+1}=2(i=1, \ldots, k-1)$. However, in practice we can simplify the CFD by taking "half" of this diagram, which ends up with $(k-1)$ vertices with $\left.\left(n_{i}, g_{i}\right)\right)=(-2,0)(i=1, \ldots, k-1)$ and a single flavor vertex with $\left(n_{k}, g_{k}\right)=(-1,0)$, while they are connected with $m_{i, i+1}=1(i=1, \ldots, k-1)$. This explains the convention of BG-CFDs for non-simply laced $G_{\mathrm{F}, \mathrm{cl}}^{5 \mathrm{~d}}$ in table 2.

2. The unmarked vertices with labels $\left(n_{i}, g_{i}\right)=(-1,0)$ will be denoted by " $(-1)$ vertices", and corresponds to an extremal curve in the geometry. A transitions between CFDs, and thus 5d SCFTs, is realized by removing such a curve. Certain extremal curves will correspond to the F-extremal weights in a gauge theory description of the SCFT.

\footnotetext{
${ }^{11}$ Geometrically, there are $p$ curves with normal bundle $\mathcal{O}+\mathcal{O}(-2)$ that are homologous in the resolved Calabi-Yau threefold.
} 
Sometimes, there will be a reducible vertex comprised of multiple $(-1)$-vertices connected to a reducible vertex containing multiple $(-2)$-vertices, which each describe homologous curves in the Calabi-Yau threefold that have to be flopped simultaneously. In the CFD language, one has to remove all the (-1)-vertices in the reducible vertex at the same time.

3. Other vertices with $n_{i}>0$, are unmarked, and are determined from the resolution of the singular geometry. However, they cannot be directly seen from the gauge theory description.

We also list the rule of CFD transitions here. After the (-1)-vertex $C_{i}$ is removed, the new graph is constructed from the original CFD with the following rules:

1. For any vertex $C_{j}$ with label $\left(n_{j}, g_{j}\right)$ that connects to $C_{i}\left(m_{i, j}>0\right)$, the updated vertex $C_{j}^{\prime}$ in the resulting CFD' has the following labels:

$$
\begin{aligned}
& n_{j}^{\prime}=n_{j}+m_{i, j}^{2} \\
& g_{j}^{\prime}=g_{j}+\frac{m_{i, j}^{2}-m_{i, j}}{2} .
\end{aligned}
$$

2. For any two vertices $C_{j}, C_{k}, j \neq k$, that connect to $C_{i}$, the new label on the edge $(j, k)$ is given by

$$
m_{j, k}^{\prime}=m_{j, k}+m_{i, j} m_{i, k}
$$

3. If there are multiple $C_{j}$ s connected to $C_{i}$, then the rule 2 applies for each pair of vertices.

The starting point of the CFD transitions is called a marginal CFD, which corresponds to a $5 \mathrm{~d}$ marginal theory that only has a UV fixed point in $6 \mathrm{~d}$. The flavor (marked) vertices in a marginal CFD can form affine Dynkin diagrams, but it is required that none of the affine Dynkin diagrams is present after any CFD transition is applied to the marginal CFD.

The $5 \mathrm{~d}$ BPS states from the M2 brane wrapping modes can be read off from the linear combinations of the vertices in the CFD. For the $5 \mathrm{~d}$ hypermultiplets, which can correspond to the matter fields in our gauge theory descriptions, they are read off from the unmarked vertices $C_{i}$ with $\left(n_{i}, g_{i}\right)=(-1,0)$.

If the SCFT has an effective gauge theory description, then its perturbative states are also formed by M2 branes wrapping certain curves that are encoded by the CFD. As will become more apparent in sections 7 and 8, these curves precisely form the BG-CFD, which therefore must be contained inside the CFD.

\subsection{Constraints on quiver gauge theories}

To determine which quiver gauge theories are consistent with any marginal CFD one can proceed in the following manner. Determine all possible embeddings of (possibly disconnected) BG-CFDs into the marginal CFD as subgraphs. These must be embedded in such a way that they are non-overlapping, and furthermore such that the marked/flavor vertices 
in any connected BG-CFD are not adjacent to the vertices of the embedding of any other connected BG-CFD. Such an embedding is necessary if we want to obtain a quiver which has a consistent classical flavor symmetry.

Since the BG-CFDs are associated to the classical flavor symmetry rotating the hypermultiplets at any flavor vertex, the BG-CFDs which can be embedded gives immediately the set of flavor symmetry groups that can be realized as rotation groups of the flavor verticess. This fixes the kinds of representations that can be realized on the flavor vertex, whether they are complex, real, or quaternionic, and also fixes the number of hypermultiplets that can appear there.

Since the CFD is, by construction, agnostic towards the details of the precise configuration of surfaces in the geometry, and thus to the details of any particular gauge sector that is disconnected from the global symmetry groups that the CFD is sensitive to, we shall find that there is often a pure-gauge ${ }^{12}$ sub-quiver in any putative quiver description. This $Q_{s}$ is generally unfixed, but of course constrained, ${ }^{13}$ however the precise details of its structure are irrelevant for the tree of descendant SCFTs, except for possible discrete dualities that depend on those details.

In addition to this we further know that the flavor rank of the SCFT must be replicated in the rank of the classical flavor symmetry of the quiver description of the marginal theory. Similarly we know the gauge rank, $r_{G}$, required of any prospective quiver from the SCFT which realizes the CFD in question. Thus we have a further constraint on quiver gauge theory descriptions from knowledge of the pair of ranks $\left(r_{G}, r_{F}\right)$.

A further set of constraints, which we refer to as the "constraints on the number of hypermultiplets", comes from the analysis of single gauge node quivers in [9]. In that paper it was shown that if a 5d single gauge node quiver was to lead to an interacting SCFT in the UV then the matter content (and where relevant the Chern-Simons level) must satisfy the following constraints: ${ }^{14}$

$$
\begin{array}{ll}
(\# \boldsymbol{S} \boldsymbol{y} \boldsymbol{m}, \# \boldsymbol{A} \boldsymbol{S}, \# \boldsymbol{F} ; k) \text { of } \mathrm{SU}(N) \leq & (1,1,1 ; 0),(1,0, N-2 ; 0),(1,0,0 ; N / 2), \\
& (0,2,8 ; 0),(0,2,7 ; 3 / 2),(0,1, N+6 ; 0), \\
& (0,1,8 ; N / 2),(0,0,2 N+4 ; 0) \\
(\# \boldsymbol{A} \boldsymbol{S}, \# \boldsymbol{F}) \text { of } \mathrm{Sp}(N) & (1,8),(0,2 N+6) \\
\# \boldsymbol{V} \text { of } \mathrm{SO}(N) & \leq N-2 \\
\# \mathbf{7} \text { of } G_{2} & \leq 6 \\
\# \mathbf{2 6} \text { of } F_{4} & \leq 3 \\
\# \mathbf{2 7} \text { of } E_{6} & \leq 4 \\
\# \mathbf{5 6} \text { of } E_{7} & \leq 3 .
\end{array}
$$

\footnotetext{
${ }^{12}$ We remind the reader that by a "pure-gauge" quiver we mean a quiver consisting only of gauge nodes — there remain bifundamental matter fields between these gauge nodes.

${ }^{13}$ For low ranks these constraints will, in fact, generally be enough to completely fix $Q_{s}$.

${ }^{14}$ We will not consider here some of the outlier options for matter representations that can appear at low rank. These are the triple anti-symmetric representations of $\mathrm{SU}(N)$ and the spinor and conjugate spinor representations of $\mathrm{SO}(N)$. The methods given throughout this paper apply with little modification to these cases also, however to prevent a proliferation of subcases we do not write of them here.
} 
When we write $\leq$ in this context we mean that the possible data associated to the gauge node must be that of a descendant of the gauge theory with the data on the right-hand side. For almost all gauge groups except $\mathrm{SU}(N)$, this is equivalent to stating that there must be fewer hypermultiplets transforming in one or more of the representations appearing on the right. In the case of $\mathrm{SU}(N)$, which has a non-trivial cubic Casimir, one must also consider how the Chern-Simons level is shifted when decoupling a hypermultiplet in a representation $\boldsymbol{R}$,

$$
k \rightarrow k \pm A(\boldsymbol{R}) / 2 .
$$

For the representations of interest in this paper the quantities $A(\boldsymbol{R})$ are

$$
A(\boldsymbol{F})=1, \quad A(\boldsymbol{A S})=N-4, \quad A(\boldsymbol{S y m})=N+4 .
$$

In this way all of the descendant gauge theories of a single gauge node quiver can be determined (one can also see them in section 3), and each individual gauge node must have the data of a descendant of one of the above right-hand side theories. In fact, for small values of the rank of the gauge algebra there are additional options. For the representations that we will consider these additional possibilities are exhausted by the following

$$
\begin{aligned}
(\# \boldsymbol{A} \boldsymbol{S}, \# \boldsymbol{F}) \text { of } \mathrm{Sp}(2) & \leq(3,0),(2,4) \\
(\# \boldsymbol{A} \boldsymbol{S}, \# \boldsymbol{F}) \text { of } \mathrm{Sp}(3) & \leq(2,0) \\
(\# \boldsymbol{F} ; k) \text { of } \mathrm{SU}(3) & \leq(6 ; 4),(3 ; 13 / 2),(0 ; 9) \\
(\# \boldsymbol{A} \boldsymbol{S}, \# \boldsymbol{F} ; k) \text { of } \mathrm{SU}(4) & \leq(4,0 ; 4),(3,4 ; 2),(3,0 ; 5),(2,0 ; 6), \\
& (1,0 ; 7),(0,8 ; 3),(0,0 ; 8) \\
(\# \boldsymbol{A S}, \# \boldsymbol{F} ; k) \text { of } \mathrm{SU}(5) & \leq(3,3 ; 0),(3,1 ; 3),(3,2 ; 3 / 2),(0,5 ; 11 / 2) \\
(\# \boldsymbol{A} \boldsymbol{S}, \# \boldsymbol{F} ; k) \text { of } \mathrm{SU}(6) & \leq(3,0 ; 3),(0,0 ; 9) .
\end{aligned}
$$

Any other gauge algebras or matter outside of that satisfying the above will not lead to an interacting SCFT as the UV fixed point of the gauge theory. This is determined by studying the Coulomb branch of the gauge theory, and as such these constraints will, in general, apply to any gauge node of an arbitrary quiver.

A further constraint is given by the positivity of the metric and the string tensions of the full quiver, which comes from study the second and first derivatives of the prepotential with respect to the Coulomb branch scalar vevs. The requirement that the metric is positive, provided that the tensions of the BPS strings do not change sign, sets sharper bounds on the possible quiver nodes and the amount of matter on them [9]. In this paper we present quivers which, based on their classical flavor symmetry embeddings in the CFDs, are possible IR description of a given CFT, and in this sense we provide a set of necessary conditions for these quivers to exist as such. It would be interesting to further restrict these bounds by studying these Coulomb branch metric constraints. We plan to come back to this general analysis in the future.

\subsection{Consistent quivers for the rank one E-string}

To illustrate this procedure of constraining weakly coupled quiver gauge theory descriptions for the SCFTs associated to a CFD, let us consider the CFD that is associated to the $6 \mathrm{~d}$ 
rank one E-string theory. By considering the marginal CFD that has a $6 \mathrm{~d}$, rather than $5 \mathrm{~d}$, SCFT as its fixed point we are constraining the possible marginal $5 \mathrm{~d}$ quiver gauge theories that flow to this $6 \mathrm{~d}$ theory in the UV. While we can of course determine possible quiver gauge theories for a non-marginal CFD we shall not do so here; this is because if a gauge theory description exists for a descendant SCFT then a similar description, with an increased number of matter hypermultiplets, should exist for the marginal theory.

In this section we will show how the three different constraints laid out in section 4.2 leads to a unique possible weakly-coupled quiver description for the rank one E-string. The marginal CFD for this theory has been determined in [1], and it is

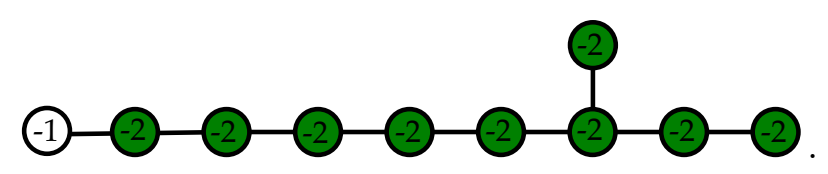

We can see that there is only exactly one way to embed disjoint unions of the BG-CFDs into the marginal CFD, that being the BG-CFD association to an $\mathrm{SO}(16)$ global symmetry group. This embedding can be drawn as

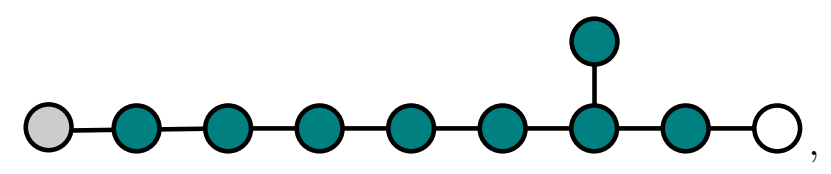

where, as in the previous section, the BG-CFD is the colored part, with turquoise denoting flavor $(-2)$-, and gray denoting (-1)-vertices. This global symmetry group can only arise from a flavor vertex associated to $m=8$ full hypermultiplets transforming in either the fundamental representation of $\operatorname{Sp}(n)$ for some $n$, or the $\mathbf{5 6}$ of $E_{7}$. However, since the rank one E-string has a single tensor multiplet, and no gauge algebras on its tensor branch in $6 \mathrm{~d}$, its $5 \mathrm{~d}$ descendants can only have a single vector multiplet coming from the reduction of the tensor. Likewise, the rank $r_{F}$ of the global gauge symmetry group is $\operatorname{rank}\left(G_{6 \mathrm{~d}}\right)+1=9$, where $G_{6 \mathrm{~d}}$ is the global symmetry group of the $6 \mathrm{~d}$ SCFT. This leaves only one option for the quiver, which is

$$
8 \boldsymbol{F}-\operatorname{Sp}(1)
$$

The final set of constraints, those coming from the number of hypermultiplets allowed for each gauge node, are not required to nail down this quiver, however we can see that the constraint

$$
\# \boldsymbol{F} \text { of } \operatorname{Sp}(n) \leq 2 n+6,
$$

is saturated for this quiver. This is, of course, nothing other than $\mathrm{SU}(2)$ with eight fundamental hypermultiplets which has long since been known to have the rank one E-string as a UV fixed point [3]. Furthermore what we show here, which is also long since known, is that this is the only possible $5 \mathrm{~d}$ quiver gauge theory description to have the rank one E-string as a UV fixed point.

In the next section we shall perform the same analysis for a variety of other, much more non-trivial, 6d SCFT starting points, and determine their heretofore unknown consistent $5 \mathrm{~d}$ quiver gauge theory descriptions. 


\subsection{Consistent quivers for $\left(D_{k}, D_{k}\right)$ minimal conformal matter}

In [1] we considered the descendants of the $6 \mathrm{~d}$ SCFT known as minimal $\left(D_{k}, D_{k}\right)$ conformal matter. Based on the known (quiver) gauge theory descriptions for theories that have this SCFT at their UV fixed point, we were able to determine a host of superconformal flavor symmetry enhancements for said theories.

In this section we will show that these quiver gauge theories descriptions discussed therein, and previously known, are found as consistent quivers satisfying the three following constraints, as laid out previously,

- the BG-CFDs for the quiver can be embedded into the marginal CFD in a nonadjacent way,

- the gauge and flavor ranks of the quiver match $\left(r_{G}, r_{F}\right)=(k-3,2 k+1)$,

- each gauge node of the quiver satisfies the constraints on the number of hypermultiplets as written in [9] and summarized in (4.6) and (4.9).

Furthermore, we will see that, up to a caveat with a reordering of the "pure-gauge" part of the quiver that we will discuss anon, the known theories saturate the options for marginal theories consistent with the above constraints. The marginal CFD, for arbitrary $k \geq 5,{ }^{15}$ was determined in [1] to be

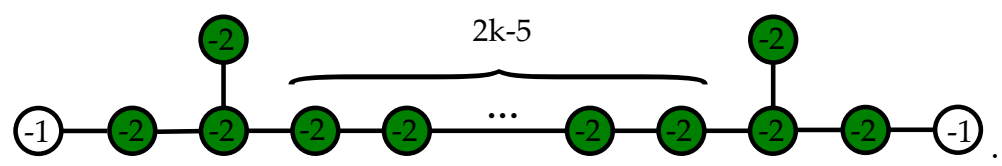

We must determine all of the possible embeddings of the BG-CFDs into this CFD. We write, for each possible embedding, the flavor symmetry group that rotates the hypermultiplets at each flavor node, for which the BG-CFD must embed, together with the subquiver associated to that embedded BG-CFD in the following table,

\begin{tabular}{|c|c|}
\hline Subgroup of $G_{\mathrm{F}, \mathrm{cl}}$ & (Sub)quivers \\
\hline $\mathrm{U}(2 k)$ & $2 k \boldsymbol{F}-\mathrm{SU}(k-2)$ \\
\hline $\mathrm{SO}(4 k)$ & $2 k \boldsymbol{F}-\mathrm{Sp}(k-2)$ \\
\hline $\mathrm{SO}(8) \times \mathrm{SO}(8)$ & $\left(4 \boldsymbol{F}-\mathrm{Sp}\left(n_{1}\right)\right) \oplus\left(4 \boldsymbol{F}-\mathrm{Sp}\left(n_{2}\right)\right)$ \\
\hline $\mathrm{SO}(8)$ & $4 \boldsymbol{F}-\mathrm{Sp}(n)$ \\
\hline
\end{tabular}

In this table we write only the part of the quiver that is directly implied by the existence of an embedding of the BG-CFD into the marginal CFD. We have also used the constraints on the number of hypermultiplets associated to any gauge node to rule out, for example, $4 \times \mathbf{5 6}-E_{7}$ from appearing. The $\oplus$ means that these are two subquivers which are part of whatever the complete quiver is that would describe this marginal theory.

\footnotetext{
${ }^{15}$ The smallest $k$ such that there is a $6 \mathrm{~d}$ SCFT is $k=4$, which however is an alternative realization of the rank one E-string discussed above.
} 
Let us consider each of these possible subquivers in turns and determine whether or not there exist complete quivers, satisfying all of the constraints, for which these are subquivers. The first example is

$$
2 k \boldsymbol{F}-\mathrm{SU}(n),
$$

for which we can see that it is not possible to add any further gauge nodes without violating the constraint on the flavor rank, $r_{F}=(2 k+1)$. As such the gauge rank constraint, $r_{G}=k-3$ fixes $n$, and we can see that the quiver

$$
2 k \boldsymbol{F}-\mathrm{SU}(k-2),
$$

is a consistent quiver for a marginal theory which has, as UV fixed point, the minimal $\left(D_{k}, D_{k}\right)$ conformal matter theory. The second possible subquiver is

$$
2 k \boldsymbol{F}-\operatorname{Sp}(n),
$$

which we can see, by exactly the same arguments as applied for the first example, that the only possible quiver containing this subquiver and satisfying all of the constraints is

$$
2 k \boldsymbol{F}-\operatorname{Sp}(k-2)
$$

We will now study all of the remaining cases simultaneously. Let us consider a subquiver, $Q_{s}$, of the full prospective quiver, where the full quiver is formed by adding either one or two flavor nodes, as in (4.15), to $Q_{s}$. We can see that $Q_{s}$ must satisfy the following properties

$$
r_{G}\left(Q_{s}\right)=r_{G}=k-3, \quad r_{F}\left(Q_{s}\right) \geq r_{F}-8=2 k-7 .
$$

The latter inequality follows as the maximal number of hypermultiplets associated to the flavor nodes is eight, as one can see from the third line of (4.15). For any quiver without loops and where each gauge node is connected to other gauge nodes by only a single (half-)hypermultiplet in the bifundamental representation, the rank of the subgroup of the global symmetry group that rotates these bifundamentals is bounded by the number of gauge nodes,

$$
n_{b}\left(Q_{s}\right) \leq n_{G}\left(Q_{s}\right)-1 .
$$

Furthermore, the bound on the rank of the gauge algebra also bounds the total number of gauge nodes of the quiver

$$
n_{G}\left(Q_{s}\right) \leq k-3 .
$$

Putting all this together we find that there are two inequalities that $Q_{s}$ must satisfy. These are

$$
\begin{aligned}
r_{F}\left(Q_{s}\right)=n_{G}\left(Q_{s}\right)+n_{b}\left(Q_{s}\right) & \leq 2 k-7, \\
r_{F}\left(Q_{s}\right) & \geq 2 k-7,
\end{aligned}
$$

for which there is only one solution: any such $Q_{s}$ must be a quiver formed out of $k-3$ gauge nodes each carrying gauge group $\operatorname{Sp}(1)$. The total quiver which may be a marginal theory for the minimal $\left(D_{k}, D_{k}\right)$ conformal matter theory is then any such $Q_{s}$ where the flavor 
nodes $4 \boldsymbol{F}$ and $4 \boldsymbol{F}$ are attached to any two distinct $\mathrm{Sp}(1)$ gauge nodes. These must be distinct nodes as otherwise the constraint that any $\mathrm{Sp}(1)$ gauge node must have at most 8 total fundamental hypermultiplets is violated. As we can see this gives a great variety of potential quiver gauge theory descriptions for the marginal theory, based on all the different configuration of the $\operatorname{Sp}(1)$ nodes in the "pure-gauge" quiver $Q_{s}$. That the quiver is not unqiue, but this is expected, as the CFD, by construction, does not contain the information about the gauge algebra; the algebra itself depends on the details of the decomposition of the reducible surface of the CFD into irreducible surfaces. This goes above and beyond the purpose of the CFD, and as such we can see that the CFD itself constrains only which flavor nodes (and thus the attached gauge nodes) can appear, and combined with the rank constraints and the constraints from the number of hypermultiplets, one can determine a set of possible quivers. This is highly restrictive, but it remains a superset of the $5 \mathrm{~d}$ quiver gauge theory descriptions which are marginal descriptions of the particular 6d SCFT under consideration.

\subsection{Consistent quivers for rank two "Model 3"}

The rank two theory that was referred to as "Model 3" in [2], i.e. $\mathrm{SU}(3)$ on a $(-1)$ curve with 12 hypers, was found to have a marginal CFD being

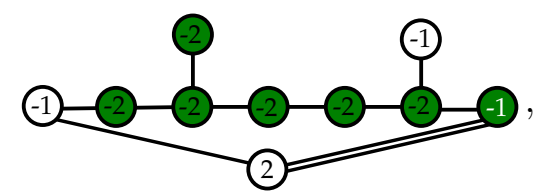

and the ranks of the gauge and classical flavor groups for any 5 d gauge theory description of this theory are known to be

$$
\left(r_{G}, r_{F}\right)=(2,7) .
$$

We again carry out the same procedure of determine possible weakly coupled gauge theory descriptions for this theory: we study all possible ways of embedding the BG-CFDs into (4.24). There are eight possible combinations of embeddings, and these are depicted in figure 14. First of all, let us just utilize the gauge rank, $r_{G}$, and the embedding of the BG-CFDs inside of the marginal CFD to write down all of the potential quivers that may be marginal descriptions of this $6 \mathrm{~d}$ SCFT. These are

$$
\begin{array}{ll}
6 \boldsymbol{F}-\mathrm{SU}(3)_{k}, & \\
m_{1} \boldsymbol{F}-\mathrm{Sp}(2)-m_{2} \boldsymbol{A S}, & m_{1}=0,4, m_{2}=0,2, \\
6 \boldsymbol{F}-\mathrm{Sp}(2), & \\
m \boldsymbol{7}-G_{2}, & m=2,6, \\
m \boldsymbol{F}-\operatorname{Sp}(1)-\operatorname{Sp}(1)_{\theta}, & m=4,6 .
\end{array}
$$




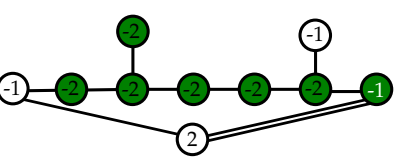

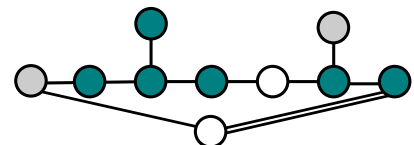

$\mathrm{SO}(8) \times \mathrm{Sp}(2)$

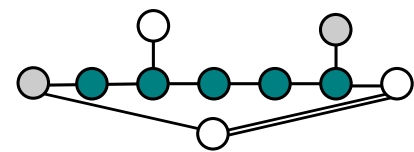

$\mathrm{U}(6)$

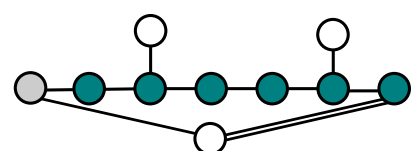

$\mathrm{Sp}(6)$

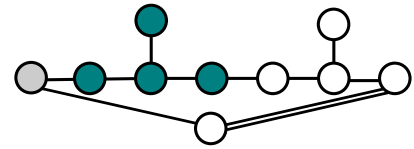

$\mathrm{SO}(8)$

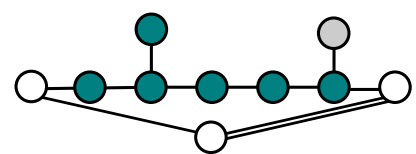

$\mathrm{SO}(12)$

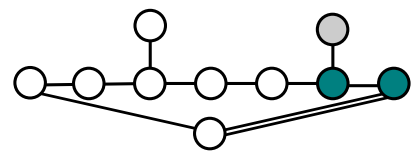

$\mathrm{Sp}(2)$

Figure 14. Embedding of BG-CFDs into the marginal CFDs of Model 3 of the rank 2 classification. From the embedding the classical flavor symmetry, and thereby $G_{\mathrm{BG}}$ can be read off.

Compatibility with the fixed flavor rank, $r_{F}=7$, leaves only five possible quivers

$$
\begin{aligned}
& 6 \boldsymbol{F}-\mathrm{SU}(3)_{k}, \\
& 4 \boldsymbol{F}-\mathrm{Sp}(2)-2 \boldsymbol{A} \boldsymbol{S}, \\
& 6 \times \boldsymbol{7}-G_{2}, \\
& 6 \boldsymbol{F}-\mathrm{Sp}(2), \\
& 4 \boldsymbol{F}-\mathrm{Sp}(1)-\mathrm{Sp}(1)_{\theta} .
\end{aligned}
$$

Of these five theories the latter two are known to be descendants of

$$
10 \boldsymbol{F}-\mathrm{Sp}(2) \quad \text { and } \quad 4 \boldsymbol{F}-\mathrm{Sp}(1)-\mathrm{Sp}(1)-4 \boldsymbol{F},
$$

respectively, which are both marginal gauge theory descriptions of minimal $\left(D_{5}, D_{5}\right)$ conformal matter. As descendants of marginal theories they cannot, in themselves, be marginal, and thus we must rule them out of the set of possible marginal quiver descriptions associated to the Model 3 CFD. This leaves only the three previously known gauge theory descriptions as the complete set of options,

$$
\begin{aligned}
& 6 \boldsymbol{F}-\mathrm{SU}(3)_{k}, \\
& 4 \boldsymbol{F}-\mathrm{Sp}(2)-2 \boldsymbol{A} \boldsymbol{S}, \\
& 6 \times \boldsymbol{7}-G_{2} .
\end{aligned}
$$

Furthermore, for the SU(3) description the Chern-Simons level can be fixed to $k=4$ by compatibility with the constraints on the number of hypermultipets as given previously.

\subsection{Consistent quivers for rank two "Model 4"}

Continuing with our study of the possible rank two quiver gauge theory descriptions of $6 \mathrm{~d}$ SCFTs, we now turn to the $6 \mathrm{~d}$ SCFT starting point referred to as "Model 4" in [2], i.e. 
$\mathrm{SU}(3)$ theory on a $(-2)$-curve with 6 hypers. There, the marginal CFD for such a CFD was determined to be

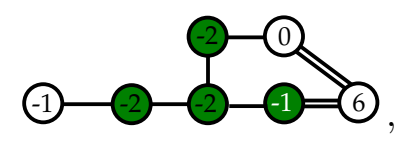

and the gauge and flavor ranks of any 5 d quiver gauge theory description are required to be

$$
\left(r_{G}, r_{F}\right)=(2,4)
$$

We can see that, after looking only at the embedding of possible BG-CFDs inside of the marginal CFD there is only one option for the embedded BG-CFD, corresponding to

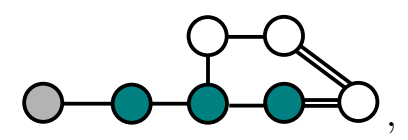

which comes from an $\mathrm{Sp}(3)$ flavor group. Restricting such that the ranks match those given above, the only possible 5d quiver gauge theory descriptions of this marginal CFT are

$$
\begin{aligned}
& 3 \boldsymbol{A} \boldsymbol{S}-\mathrm{Sp}(2), \\
& 3 \times \mathbf{7}-G_{2} .
\end{aligned}
$$

The latter quiver is a descendant of $6 \times \mathbf{7}-G_{2}$, which is a known marginal theory describing Model 3. As such it cannot be associated to a marginal theory, and thus our analysis leaves the only possible quiver description $3 \boldsymbol{A} \boldsymbol{S}-\mathrm{Sp}(2)$ for Model 4 .

\subsection{Consistent quivers for $\left(E_{6}, E_{6}\right)$ minimal conformal matter}

Let us now consider the case of minimal $\left(E_{6}, E_{6}\right)$ conformal matter. As before, to determine possible quiver descriptions of the marginal theory it is of the first importance to determine which possible BG-CFDs associated to any classical flavor group can be embedded into the marginal CFD. As it turns out, there are a limited number of options. Since the marginal CFD is simply-laced, in the sense that all of the marked green vertices have label $n=-2$, the only types of connected BG-CFDs that can be embedded are those associated to $\mathrm{U}(k)$ and $\mathrm{SO}(2 k)$ flavor factors.

The marginal CFD was determined in $[1,2]$ from geometric considerations, and it is

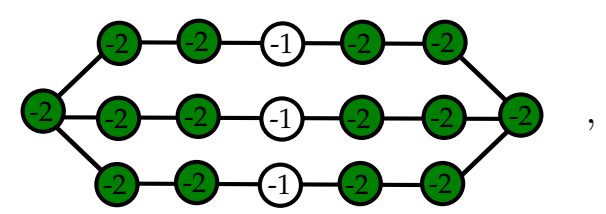

and only allows gauge descriptions with gauge and flavor ranks given by

$$
\left(r_{G}, r_{F}\right)=(5,13)
$$

All of the different possible embeddings are shown in figure 15 . We see immediately that if there is a $\mathrm{U}(k)$ factor in the classical flavor group then there can only be one such factor, 


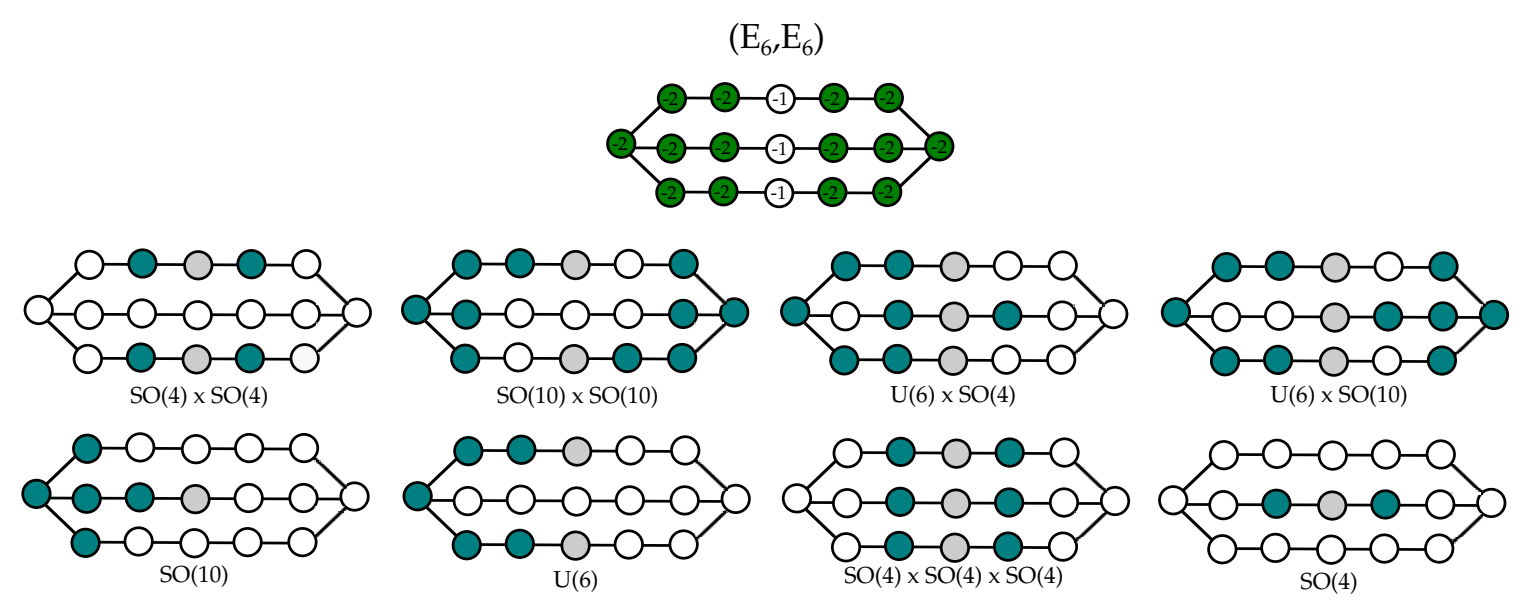

Figure 15. Embedding of potential BG-CFDs into the $\left(E_{6}, E_{6}\right)$ marginal CFD. Below each embedding of the BG-CFD into the marginal CFD we list the classical flavor symmetry.

and furthermore it must have $k=6$; it is apparent even from this immediate result that the structure of the marginal CFD powerfully constrains possible quiver gauge theories that have minimal $\left(E_{6}, E_{6}\right)$ conformal matter as a UV fixed point.

Let us now combine this analysis with the full set of constraints that were previously described. The possible flavor nodes of any quiver description are one of the following

$$
\begin{array}{ll}
6 \boldsymbol{F}-\mathrm{SU}(n), & \\
\left(6 \boldsymbol{F}-\mathrm{SU}\left(n_{1}\right)\right) \oplus\left(m \boldsymbol{F}-\mathrm{Sp}\left(n_{2}\right)\right), & m=2,5, \\
m \boldsymbol{F}-\mathrm{Sp}(n), & m=2,5, \\
\left(m \boldsymbol{F}-\mathrm{Sp}\left(n_{1}\right)\right) \oplus\left(m \boldsymbol{F}-\mathrm{Sp}\left(n_{2}\right)\right), & m=2,5, \\
\left(2 \boldsymbol{F}-\mathrm{Sp}\left(n_{1}\right)\right) \oplus\left(2 \boldsymbol{F}-\mathrm{Sp}\left(n_{2}\right)\right) \oplus\left(2 \boldsymbol{F}-\mathrm{Sp}\left(n_{3}\right)\right), &
\end{array}
$$

We will now write down some explicit quivers with the maximal rank of the global symmetry group rotating the hypermultiplets at each flavor nodes. Such a quiver will extend down the maximal depth inside of the CFD tree, and thus have a wide variety of $5 \mathrm{~d}$ SCFTs at the UV fixed points of its descendants. It is immediately clear that the subquiver corresponding to

$$
\left(6 \boldsymbol{F}-\mathrm{SU}\left(n_{1}\right)\right) \oplus\left(5 \boldsymbol{F}-\mathrm{Sp}\left(n_{2}\right)\right),
$$

cannot be combined into a quiver satisfying $r_{F}=13$. The maximal quivers that we can attempt to determine must be constructed from the subquiver option

$$
\left(5 \boldsymbol{F}-\operatorname{Sp}\left(n_{1}\right)\right) \oplus\left(5 \boldsymbol{F}-\operatorname{Sp}\left(n_{2}\right)\right) .
$$

One option is to consider the quiver

$$
5 \boldsymbol{F}-\mathrm{Sp}\left(n_{1}\right)-\mathrm{Sp}\left(n_{2}\right)-5 \boldsymbol{F},
$$


which has the correct global symmetry group rank. The constraints that such a quiver is consistent with the gauge rank and with the constraints on the number of hypermultiplets are

$$
\begin{array}{ll}
\# \boldsymbol{F} \text { of } \operatorname{Sp}\left(n_{1}\right): & 2 n_{2}+5 \leq 2 n_{1}+6 \\
\# \boldsymbol{F} \text { of } \operatorname{Sp}\left(n_{2}\right): & 2 n_{1}+5 \leq 2 n_{2}+6 \\
r_{G}=5: & n_{1}+n_{2}=5,
\end{array}
$$

for which it is straightforward to see that there is no solution. The second possible quiver is

$$
5 \boldsymbol{F}-\operatorname{Sp}\left(n_{1}\right)-G-\operatorname{Sp}\left(n_{2}\right)-5 \boldsymbol{F},
$$

where $G$ has a real (for consistency with $r_{F}=13$ ) even-dimensional (for consistency with the anomaly requiring $S p$ gauge algebras to have an even number of fundamental half-hypermultiplets) fundamental representation. Furthermore, the rank of $G$ must be $\operatorname{rank}(G) \leq 3$ ) for consistency with $r_{G}=5$. This leaves precisely one option, which is indeed a consistent quiver description satisfying all of the constraints,

$$
5 \boldsymbol{F}-\mathrm{Sp}(1)-\mathrm{SO}(6)-\mathrm{Sp}(1)-5 \boldsymbol{F} .
$$

This is the only possible maximal depth quiver which may be a marginal theory for $\left(E_{6}, E_{6}\right)$ minimal conformal matter.

\subsection{Consistent quivers for $\left(E_{7}, E_{7}\right)$ minimal conformal matter}

In this subsection we will consider potential marginal quiver gauge theories that flow to $6 \mathrm{~d}$ minimal $\left(E_{7}, E_{7}\right)$ conformal matter at the UV fixed point. Any such theory must satisfy

$$
\left(r_{G}, r_{F}\right)=(10,15)
$$

and the marginal CFD for this CFT is known to be [2]

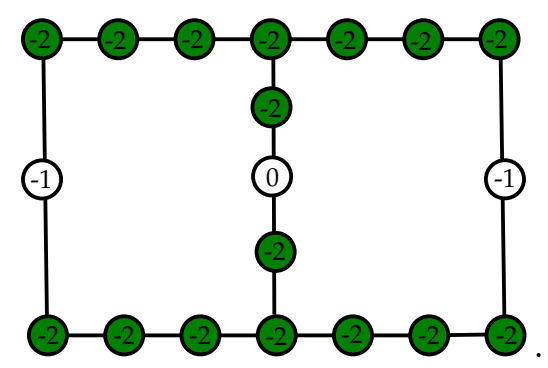

All possible combinations of embedding the BG-CFDs into this marginal CFD are shown in figure 16. The possible flavor nodes of the quivers can be completely classified by the possible embeddings of the BG-CFDs into the marginal CFD, and the following options 
$\left(\mathrm{E}_{7}, \mathrm{E}_{7}\right)$

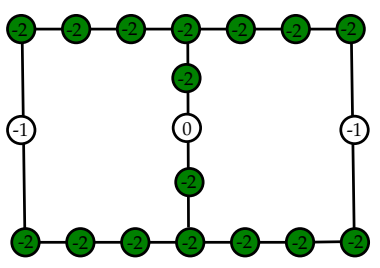

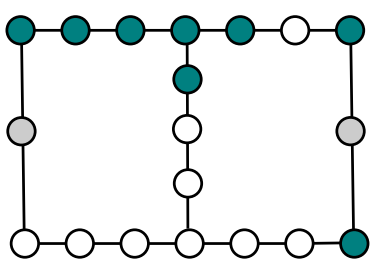

$\mathrm{SO}(12) \times \mathrm{SO}(4)$

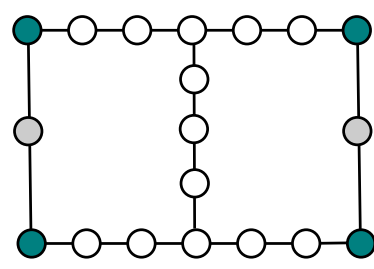

$\mathrm{SO}(4) \times \mathrm{SO}(4)$

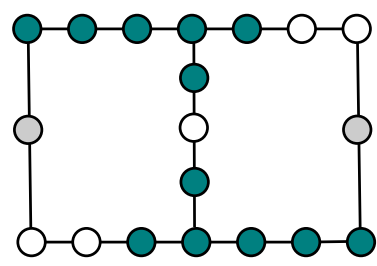

$\mathrm{SO}(12) \times \mathrm{SO}(12)$

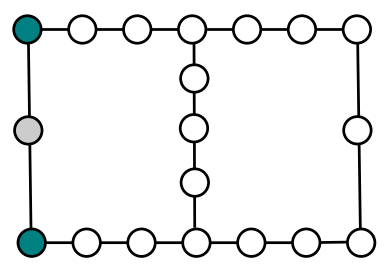

$\mathrm{SO}(4)$

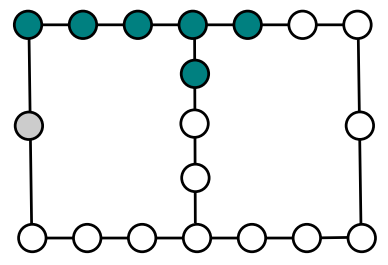

$\mathrm{SO}(12)$

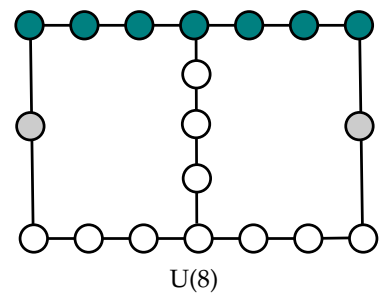

Figure 16. Embedding of potential BG-CFDs into the $\left(E_{7}, E_{7}\right)$ Marginal CFD.

for the flavor nodes are found:

$$
\begin{array}{ll}
8 \boldsymbol{F}-\mathrm{SU}(n), & \\
m \boldsymbol{F}-\mathrm{Sp}(n), & m=2,6, \\
\left(m_{1} \boldsymbol{F}-\operatorname{Sp}\left(n_{1}\right)\right) \oplus\left(m_{2} \boldsymbol{F}-\operatorname{Sp}\left(n_{2}\right)\right), & m_{i} \in\{2,6\}, \\
2 \times \mathbf{5 6}-E_{7}, & \\
\left(m \boldsymbol{F}-\operatorname{Sp}\left(n_{1}\right)\right) \oplus\left(2 \times \mathbf{5 6}-E_{7}\right), & m=2,6 .
\end{array}
$$

There are many quivers which can be found with these as the flavor nodes, which also satisfy the constraints imposed by $r_{G}, r_{F}$, and those described earlier as the constraints on the number of hypermultiplets. The enumeration of all such quivers is unenlightening, however, we can determine some interesting potential quiver descriptions. One kind of description of interest, as in the $\left(E_{6}, E_{6}\right)$ case just discussed, is a quiver with the maximal number of matter hypermultiplets associated to flavor nodes. Here this would be any quivers associated to

$$
\left(6 \boldsymbol{F}-\operatorname{Sp}\left(n_{1}\right)\right) \oplus\left(6 \boldsymbol{F}-\operatorname{Sp}\left(n_{2}\right)\right) .
$$

There are only two possibilities to connect these two subquivers together while satisfying the constraint from $r_{F}$ and that is either to write

$$
6 \boldsymbol{F}-\operatorname{Sp}\left(n_{1}\right)-\operatorname{Sp}\left(n_{2}\right)-6 \boldsymbol{F},
$$


or

$$
6 \boldsymbol{F}-\operatorname{Sp}\left(n_{1}\right)-G-\operatorname{Sp}\left(n_{2}\right)-6 \boldsymbol{F},
$$

where $G$ has a real fundamental representation. We consider the case of (4.47) first. The constraints on the number of hypermultiplets require that

$$
\begin{aligned}
& 2 n_{2}+6 \leq 2 n_{1}+6 \\
& 2 n_{1}+6 \leq 2 n_{2}+6,
\end{aligned}
$$

and, together with the gauge rank constraint that

$$
n_{1}+n_{2}=10,
$$

one finds that the only possible solution is

$$
6 \boldsymbol{F}-\mathrm{Sp}(5)-\mathrm{Sp}(5)-6 \boldsymbol{F} .
$$

Now we turn to maximal depth quivers of the form (4.48). It is immediate to see that $G$ cannot be either of $\mathrm{SO}(2 k+1)$ or $G_{2}$ as these groups have odd-dimensional fundamental representations, which is incompatible with the anomaly requirement that $\operatorname{Sp}(n)$ gauge groups must come with an even number of fundamental hypermultiplets. If we take $G=F_{4}$ then the constraints on the number of hypermultiplets become

$$
\begin{array}{lrlrl}
\text { \# } \boldsymbol{F} \text { of } \operatorname{Sp}\left(n_{1}\right): & 6+\frac{1}{2} \times 26 & \leq 2 n_{1}+6 \\
\text { \# } \boldsymbol{F} \text { of } \operatorname{Sp}\left(n_{2}\right): & 6+\frac{1}{2} \times 26 & \leq 2 n_{2}+6 \\
\text { \# } 26 \text { of } F_{4}: & \frac{1}{2} \times\left(2 n_{1}+2 n_{2}\right) & \leq 3,
\end{array}
$$

which, together with the gauge rank constraint that $n_{1}+n_{2}=6$, clearly has no solutions. If we instead consider the case where $G=\mathrm{SO}(2 r)$ then the set of constraints is

$$
\begin{aligned}
\# \boldsymbol{F} \text { of } \operatorname{Sp}\left(n_{1}\right): & 6+r & \leq 2 n_{1}+6 \\
\# \boldsymbol{F} \text { of } \operatorname{Sp}\left(n_{2}\right): & 6+r & \leq 2 n_{2}+6 \\
\text { \#F of } \mathrm{SO}(2 r): & n_{1}+n_{2} & \leq 2 r-2 \\
r_{G}=10: & n_{1}+n_{2}+r & =10 .
\end{aligned}
$$

This system of equations has only two solutions

$$
\left(n_{1}, n_{2}, r\right)=(3,3,4) \quad \text { or } \quad(2,4,4) .
$$

In short, we deduce that there are three potential marginal quiver gauge theories for minimal $\left(E_{7}, E_{7}\right)$ conformal matter which have the maximum rank of the flavor nodes. These are

$$
\begin{aligned}
& 6 \boldsymbol{F}-\mathrm{Sp}(5)-\mathrm{Sp}(5)-6 \boldsymbol{F} \\
& 6 \boldsymbol{F}-\mathrm{Sp}(3)-\mathrm{SO}(8)-\mathrm{Sp}(3)-6 \boldsymbol{F} \\
& 6 \boldsymbol{F}-\mathrm{Sp}(2)-\mathrm{SO}(8)-\mathrm{Sp}(4)-6 \boldsymbol{F} .
\end{aligned}
$$




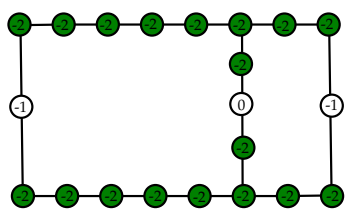

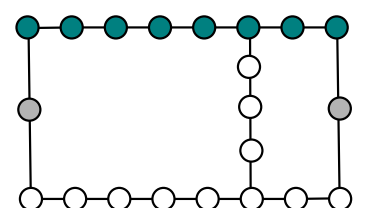

$\mathrm{U}(9)$

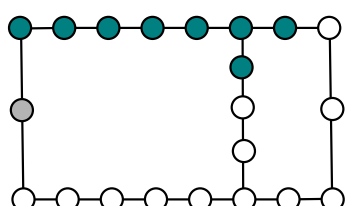

$\mathrm{SO}(16)$

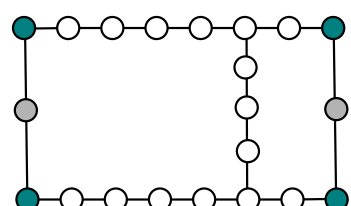

$\mathrm{SO}(4) \times \mathrm{SO}(4)$

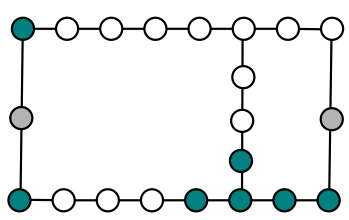

$\mathrm{SO}(4) \times \mathrm{SO}(10)$

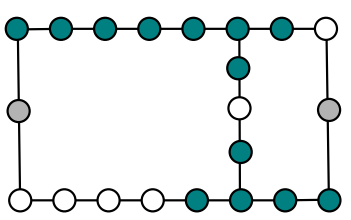

$\mathrm{SO}(16) \times \mathrm{SO}(10)$

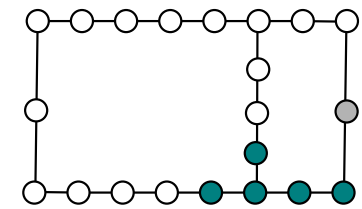

$\mathrm{SO}(10)$

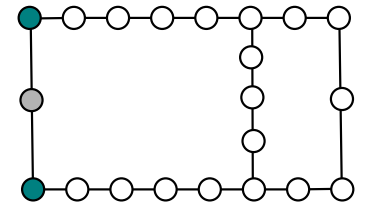

$\mathrm{SO}(4)$

Figure 17. Embedding of potential BG-CFDs into the $\left(E_{8}, E_{8}\right)$ Marginal CFD.

\subsection{Consistent quivers for $\left(E_{8}, E_{8}\right)$ minimal conformal matter}

Minimal $\left(E_{8}, E_{8}\right)$ conformal matter is a 6 d SCFT with associated marginal CFD [2]

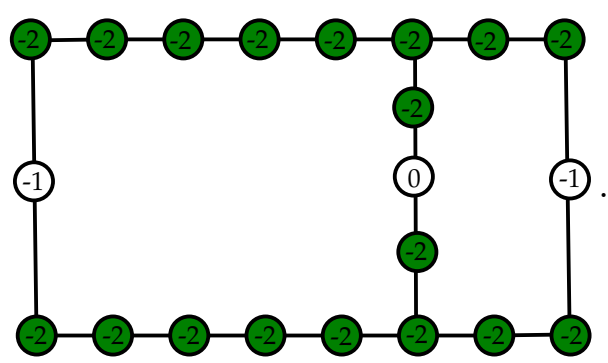

Any $5 \mathrm{~d}$ quiver gauge theory that realizes minimal $\left(E_{8}, E_{8}\right)$ conformal matter at its UV fixed point must have the rank of the gauge algebra and the rank of the classical flavor symmetry being

$$
\left(r_{G}, r_{F}\right)=(21,17) \text {. }
$$

All of the possible embeddings of the BG-CFDs into this marginal CFD are given in figure 17, and from this one can determine that the possible flavor nodes of any quiver that may be a marginal theory for minimal $\left(E_{8}, E_{8}\right)$ conformal matter are the following

$$
\begin{array}{lr}
9 \boldsymbol{F}-\mathrm{SU}(n), & \\
m \boldsymbol{F}-\mathrm{Sp}(n), & m=2,5,8, \\
2 \times \mathbf{5 6}-E_{7}, & \\
\left(m_{1} \boldsymbol{F}-\mathrm{Sp}\left(n_{1}\right)\right) \oplus\left(m_{2} \boldsymbol{F}-\mathrm{Sp}\left(n_{2}\right)\right), & \left(m_{1}, m_{2}\right)=(2,2),(2,5),(5,8), \\
\left(m \boldsymbol{F}-\mathrm{Sp}\left(n_{1}\right)\right) \oplus\left(2 \times \mathbf{5 6}-E_{7}\right), & m=2,5, \\
\left(2 \times \mathbf{5 6}-E_{7}\right) \oplus\left(2 \times \mathbf{5 6}-E_{7}\right) . &
\end{array}
$$


As in the other cases of $\left(E_{n}, E_{n}\right)$ conformal matter, we will be interested in determining precise possible quivers for the theories which have the deepest descendants; that is, the quivers for which a gauge theory description exists farthest down the CFD-tree. In this case such a quiver is one with flavor nodes

$$
\left(8 \boldsymbol{F}-\operatorname{Sp}\left(n_{1}\right)\right) \oplus\left(5 \boldsymbol{F}-\operatorname{Sp}\left(n_{2}\right)\right) .
$$

There are two possible options to connect this into a complete quiver satisfying that $r_{F}=17$. The first option is to connect the two $\operatorname{Sp}(n)$ factors via a bifundamental hypermultiplet, and add an additional gauge node connecting to one of the $\operatorname{Sp}(n)$ in such a way that the bifundamental gluing those two nodes is quaternionic, and thus does not provide any additional classical flavor symmetry. A straightforward analysis from the constraints on the number of hypermultiplets shows that such a quiver does not have an interacting 5d SCFT fixed point.

There are then three possible options if we consider only quivers without loops, and where each gauge node is glued to another gauge node by only a single bifundamental (half-)hypermultiplet. One of these is

$$
\begin{gathered}
G_{(2)} \\
8 \boldsymbol{F}-\operatorname{Sp}\left(n_{1}\right)-G_{(1)}-\operatorname{Sp}\left(n_{2}\right)-5 \boldsymbol{F}
\end{gathered} .
$$

The other options involve attaching the group $G_{(2)}$ to one of the $\operatorname{Sp}\left(n_{i}\right)$ factors instead of $G_{(1)}$, however, for brevity, we shall not consider those options here. $G_{(1)}$ is required to be a group with a real, even-dimensional, fundamental representation and $G_{(2)}$ one with a quaternionic fundamental representation. It is straightforward to see that neither of these groups can consistently be exceptional groups, and the only option is

$$
\left(G_{(1)}, G_{(2)}\right)=\left(\mathrm{SO}\left(2 r_{1}\right), \mathrm{Sp}\left(r_{2}\right)\right) .
$$

Such a quiver satisfies the constraints coming from $r_{F}=17$ and the remaining constraints are

$$
\begin{array}{lrl}
\# \boldsymbol{F} \text { of } \operatorname{Sp}\left(n_{1}\right): & 8+r_{1} & \leq 2 n_{1}+6, \\
\# \boldsymbol{F} \text { of } \operatorname{Sp}\left(n_{2}\right): & 5+r_{1} & \leq 2 n_{2}+6, \\
\# \boldsymbol{F} \text { of } \mathrm{SO}\left(2 r_{1}\right): & n_{1}+n_{2}+r_{2} & \leq 2 r_{1}-2, \\
\# \boldsymbol{F} \text { of } \mathrm{Sp}\left(r_{2}\right): & r_{1} & \leq 2 r_{2}+6, \\
r_{G}=21: & n_{1}+n_{2}+r_{1}+r_{2} & =21 .
\end{array}
$$

There are many explicit quivers that exist as solutions of these constraints. Therefore there are potential maximal depth quivers for minimal $\left(E_{8}, E_{8}\right)$ conformal matter of the form

$$
\begin{gathered}
\operatorname{Sp}\left(r_{2}\right) \\
\mid \\
8 \boldsymbol{F}-\operatorname{Sp}\left(n_{1}\right)-\operatorname{SO}\left(2 r_{1}\right)-\operatorname{Sp}\left(n_{2}\right)-5 \boldsymbol{F}
\end{gathered}
$$


where

$$
\begin{aligned}
\left(n_{1}, n_{2}, r_{1}, r_{2}\right)= & (5,4,8,4),(5,5,8,3),(5,7,8,1),(5,6,8,2), \\
& (6,4,8,3),(6,5,8,2),(6,6,8,1),(7,4,8,2), \\
& (7,5,8,1),(8,4,8,1),(6,4,9,2) .
\end{aligned}
$$

\subsection{Consistent quivers for $\left(E_{8}, \mathrm{SU}(2 k+1)\right)$ minimal conformal matter}

The marginal CFD for $\left(E_{8}, \mathrm{SU}(2 k+1)\right)$ minimal conformal matter, which is a theory with

$$
\left(r_{G}, r_{F}\right)=\left(2 k^{2}+k+1,2 k+9\right),
$$

was determined in appendix D of [2]. There it was found to be

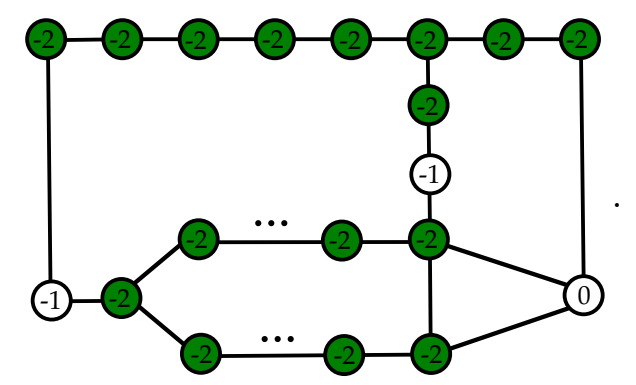

By studying the embeddings of the BG-CFDs in this marginal CFD one can again determine that the possible flavor nodes of any quiver are

$$
\begin{array}{ll}
m \boldsymbol{F}-\mathrm{SU}(n), & m=8, k+2, k+3, \\
m \boldsymbol{A} \boldsymbol{S}-\mathrm{SU}(n), & m=k+2, k+3, \quad(\text { if } k=1,2), \\
m \mathbf{2 7}-E_{6}, & m=k+2, k+3, \quad k \leq 2, \\
m \boldsymbol{F}-\mathrm{Sp}(n), & m=2,3,4,8, \quad(m=3 \text { only if } k>1), \\
m \mathbf{5 6}-E_{7}, & m=2,3, \quad(m=3 \text { only if } k>1), \\
\left(m_{1} \boldsymbol{F}-\mathrm{Sp}\left(n_{1}\right)\right) \oplus\left(m_{2} \boldsymbol{F}-\mathrm{Sp}\left(n_{2}\right)\right), & m_{1} \in\{2,3\}, m_{2} \in\{2,3,4\}, \\
\left(m \boldsymbol{F}-\mathrm{Sp}\left(n_{1}\right)\right) \oplus\left(2 \times \mathbf{5 6}-E_{7}\right), & m=2,3,4, \\
\left(2 \times \mathbf{5 6}-E_{7}\right) \oplus\left(2 \times \mathbf{5 6}-E_{7}\right) . &
\end{array}
$$

In the latter three quivers the general values of $m_{i}$ given are only potential options for sufficiently large $k$. We find that the following are not allowed

$$
\begin{aligned}
& \left(m_{1}, m_{2}\right) \text { or }\left(m_{2}, m_{1}\right) \neq(3,4),(2,2) \quad \text { when } k \leq 1, \\
& \text { when } k \leq 2 \text {, } \\
& \text { when } k \leq 3 \text {. }
\end{aligned}
$$

Let us now write down some explicit potential quivers for the marginal theory associated to $\left(E_{8}, \mathrm{SU}(2 k+1)\right)$ minimal conformal matter for some particular small values of $k$. We 
will take $k=1$, which was discussed from the geometric point of view in [2]. In fact $6 \mathrm{~d}$ theory has a further enhancement of the superconformal flavor symmetry, to $E_{8} \times G_{2}$, and thus to determine the full superconformal flavor symmetry of the descendants it was useful to introduce a different marginal CFD that captured this enhancement. Of course, by studying the $\left(E_{8}, \mathrm{SU}(3)\right)$ marginal $\mathrm{CFD}$ and its descendants one can still determine a non-trivial enhancement of the superconformal flavor symmetry directly from the CFD, and when computing the BPS spectrum, as in [2], one observes that the states organize into representations of the larger flavor symmetry group. This is to say, a potential quiver derived from the $\left(E_{8}, \mathrm{SU}(3)\right)$ marginal $\mathrm{CFD}$ is a necessary condition for the quiver to describe the marginal theory, regardless of the further symmetry enhancement.

We are considering a theory with

$$
\left(r_{G}, r_{F}\right)=(4,11)
$$

We now attempt to determine a quiver with the maximal depth of descendants. After little reflection one can see that any quiver with a flavor node charged under an $\mathrm{SU}(n \geq 3)$ gauge group cannot be consistent with the ranks and the constraints on the number of hypermultiplets. The maximal quiver would then involve an $\operatorname{Sp}(n)$ gauge node with $8 \boldsymbol{F}$, and it is straightforward to see that there is only one such possible quiver, being

$$
8 \boldsymbol{F}-\operatorname{Sp}(3)-\operatorname{Sp}(1)_{\theta}
$$

Furthermore, if one is interested in quivers corresponding to the flavor nodes

$$
\left(m_{1} \boldsymbol{F}-\operatorname{Sp}\left(n_{1}\right)\right) \oplus\left(m_{2} \boldsymbol{F}-\operatorname{Sp}\left(n_{2}\right)\right)
$$

then one can determine that the only options are when $m_{1}=4$ and $m_{2}=2$, for which there are precisely nine different complete quivers satisfying all of the consistency requirements.

\subsection{Consistent quivers for $\left(E_{8}, \mathrm{SU}(2 k)\right)$ minimal conformal matter}

The marginal CFD for minimal $\left(E_{8}, \mathrm{SU}(2 k)\right)$ conformal matter is [2]

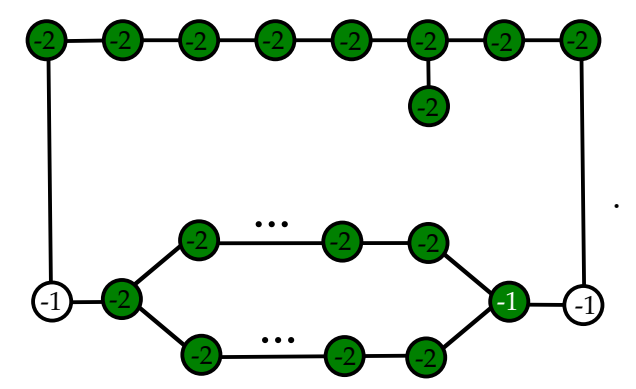

Any 5d quiver gauge theory description that flows in the UV to this 6d SCFT must have

$$
\left(r_{G}, r_{F}\right)=\left(2 k^{2}-k+1,2 k+8\right)
$$


By determining all of the possible embeddings of BG-CFDs into the marginal CFD one finds the following set of possible flavor nodes for $5 \mathrm{~d}$ quiver descriptions of this $6 \mathrm{~d}$ SCFT

$$
\begin{array}{ll}
9 \boldsymbol{F}-\mathrm{SU}(n), & m=2,3,5,8, \\
m \boldsymbol{F}-\mathrm{Sp}(n), & m=2,3, \\
m \mathbf{5 6}-E_{7}, & m=2,3,8, \\
m \boldsymbol{F}-\mathrm{Sp}(n)-1 \boldsymbol{A S}, & m=2,3,8, \\
(m \boldsymbol{F}-\mathrm{Sp}(n)) \oplus(1 \boldsymbol{A S}-\mathrm{Sp}(r)), & \\
(m \boldsymbol{F}-\mathrm{Sp}(n)) \oplus(1 \boldsymbol{V}-\mathrm{SO}(r)), & m=2,3,8, \\
(m \boldsymbol{F}-\mathrm{Sp}(n)) \oplus\left(1 \times \mathbf{7}-G_{2}\right), & m=2,3,8, \\
(m \boldsymbol{F}-\mathrm{Sp}(n)) \oplus\left(1 \times \mathbf{2 6}-F_{4}\right), & m=2,3,8, \\
\left(2 \times \mathbf{5 6}-E_{7}\right) \oplus(1 \boldsymbol{A S}-\mathrm{Sp}(r)), & \\
\left(2 \times \mathbf{5 6}-E_{7}\right) \oplus(1 \boldsymbol{V}-\mathrm{SO}(r)), & \\
\left(2 \times \mathbf{5 6}-E_{7}\right) \oplus\left(1 \times \mathbf{7}-G_{2}\right), & \\
\left(2 \times \mathbf{5 6}-E_{7}\right) \oplus\left(1 \times \mathbf{2 6}-F_{4}\right), & \\
k \boldsymbol{A S}-\mathrm{Sp}(r), & \\
k \boldsymbol{V}-\mathrm{SO}(r), & \\
k \mathbf{7}-G_{2}, & \\
k \mathbf{2 6}-F_{4}, & \\
\left(5 \boldsymbol{F}-\mathrm{Sp}\left(n_{1}\right)\right) \oplus\left(m \boldsymbol{F}-\mathrm{Sp}\left(n_{2}\right)\right), 3, \\
\left(5 \boldsymbol{F}-\mathrm{Sp}\left(n_{1}\right)\right) \oplus\left(2 \times \mathbf{5 6}-E_{7}\right) .
\end{array}
$$

Furthermore, quivers with classical flavor symmetry

$$
\mathrm{SO}(10) \times \mathrm{SO}(6) \quad \text { or } \quad \mathrm{SO}(6) \quad \text { or } \quad \mathrm{SO}(4) \times \mathrm{Sp}(1)
$$

rotating the hypermultiplets attached to the flavor nodes requires one to have

$$
k>1
$$

and similarly we find that

$$
\mathrm{SO}(6) \times \mathrm{Sp}(1) \quad \text { requires } \quad k>2 \text {. }
$$


When $k=1$ this $6 \mathrm{~d} \mathcal{N}=(1,0)$ SCFT is the rank two E-string theory, for which $5 \mathrm{~d}$ quiver gauge theory descriptions are known. We can now reproduce this set of descriptions from our approach, and thus show that all possibilities are realized. It is immediate on checking all options in (4.74) that the only potential quiver descriptions, consistent with the gauge and flavor ranks, together with the constraints on the number of hypermultiplets are

$$
\begin{aligned}
& 9 \boldsymbol{F}-\mathrm{SU}(3) \\
& 8 \boldsymbol{F}-\mathrm{Sp}(2)-1 \boldsymbol{A} \boldsymbol{S} \\
& 5 \boldsymbol{F}-\mathrm{Sp}(1)-\mathrm{Sp}(1)-2 \boldsymbol{F} .
\end{aligned}
$$

These are exactly the set of known quiver gauge theory descriptions that have the rank two E-string as their UV fixed point.

We stress that while all potential quivers are realized for ranks one and two 6d SCFTs, we do not expect this to generalize to higher ranks - it is essentially an accident that the low rank combined with the restrictions on the flavor nodes is exceptionally constraining. As we have seen, for higher ranks in general there are many more potential quivers than there are known gauge theory descriptions. It remains to determine which of these quivers are, in fact, realized, however we do not expect that a pure CFD approach is capable of answering this question. The CFD is, by definition, defined in terms of a reducible surface, and the details of the "pure gauge" part of any quiver description is contained precisely inside of the details of how that reducible surface is glued together from irreducible surfaces. However, at all ranks we can see that the embedding of the BG-CFDs inside of the marginal CFD is extremely constraining on what possible flavor nodes can appear in any quiver description.

\section{Bootstrapping CFDs}

\subsection{Constraining marginal CFDs of single gauge node theories}

In this section, we demonstrate the power of BG-CFDs as an alternative approach to constrain and "derive" the actual CFDs. In certain instances, there is no known geometric realization of the marginal theory in 6d F-theory language. In such instances, we can nevertheless 'bootstrap' the marginal CFD using consistency requirements with known properties.

1. The marginal CFD has a marked subgraph, which is given by the Dynkin diagram of the $6 \mathrm{~d}$ superconformal flavor symmetry (generically these will be affine Dynkin diagrams).

2. The rules for constructing CFD-descendants need to work in parallel with the mass deformations. Along with the known superconformal flavor symmetries, this condition will largely fix the location of $(-1)$-vertices in the marginal CFD.

3. The classical flavor symmetry determines a set of BG-CFDs, which have to be embeddable into the CFDs. If there are multiple non-Abelian factors, the corresponding BG-CFDs cannot intersect each other. This rule also applies to the marginal CFD.

4. Applying any mass deformation (i.e. CFD-transition to any $(-1)$-vertex) to the marginal CFD has to result in a CFD, whose marked sub-graph is a Dynkin diagram (without any affine marked subgraphs). 
These conditions do not necessarily constrain the CFDs entirely and uniquely, but they give at worst a subgraph that encodes a subset of mass-deformations. Nonetheless, we will try to reconstruct the conjectural marginal CFD in this way.

In this section, we will focus on the subclass of marginal theories which have a description in terms of a gauge theory with a simple gauge algebra $\mathrm{SU}(N),(N \geq 4)$, which has the matter contents of $2 \boldsymbol{A S}+8 \boldsymbol{F}$ and the following 6 d tensor branch [9]:

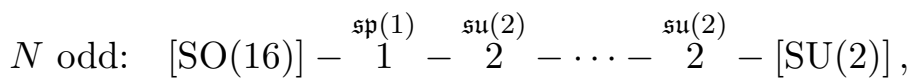

$$
\begin{aligned}
& N \geq 6 \text { even: } \quad\left[E_{7}\right]-1-\underset{[\mathrm{SU}(2)]}{2}-\stackrel{\mathfrak{s u}(2)}{2}-\stackrel{\mathfrak{s u}(2)}{2}-\cdots-{ }^{\mathfrak{s u}(2)}-[\mathrm{SU}(2)] \\
& N=4: \quad\left[E_{7}\right]-1-\stackrel{\mathfrak{s u}(2)}{2}-[\mathrm{SO}(7)] .
\end{aligned}
$$

The $6 \mathrm{~d}$ flavor symmetries are $[9,69]$

$$
\begin{aligned}
N \geq 5 \text { odd: } & \mathrm{SO}(16) \times \mathrm{SU}(2)^{2} \\
N \geq 6 \text { even: } & E_{7} \times \mathrm{SU}(2)^{3} \\
N=4: & E_{7} \times \mathrm{SO}(7)
\end{aligned}
$$

For $N \geq 5$, there is an extra $\mathrm{SU}(2)$ flavor symmetry at the $6 \mathrm{~d}$ superconformal point comparing to the tensor branches in (5.1). This is related to the unique linear combination of the baryonic $\mathrm{SU}(2) \mathrm{s}$, which remains non-anomalous.

The $N=4$ case corresponds to the $\left(E_{7}, \mathrm{SO}(7)\right)$ conformal matter theory. For $N>4$, they do not have a known singular Weiertrass model in the $6 \mathrm{~d}$ F-theory description, and we will apply the bootstrap methodology to get a conjectural marginal CFD. Finally, we see that the resulting marginal CFD has in general more descendants than those realized by known gauge theory descriptions, which are indicators for dual gauge/quiver descriptions. Such quiver gauge theory descriptions and dualities are extensively discussed in section 6 .

\section{$5.2 \mathrm{SU}(2 n)_{0}+2 A S+8 F$}

Consider the marginal theory

$$
\mathrm{SU}(2 n)_{0}+2 \boldsymbol{A} \boldsymbol{S}+8 \boldsymbol{F}, \quad n>2 .
$$

The classical flavor symmetry is $\mathrm{U}(2) \times \mathrm{U}(8)$, and the associated BG-CFD needs to embed into the marginal CFD.

In the following it will be useful to recall some of the known flavor symmetry enhancement for $5 \mathrm{~d} \mathrm{SU}(N)$ gauge theories with $N_{A} \boldsymbol{A S}+N_{f} \boldsymbol{F}$ matter fields at the their UV fixed points. For $N_{A}=2$ and $N_{f} \leq 8$, the flavor symmetry enhancements were determined in the appendix of [21]. For the cases of $N_{A}=1$, the UV flavor symmetry enhancements are implicitly given in [33], and we summarize them here explicitly in appendix B. Finally, for the cases of $N_{A}=0$, the superconformal flavor symmetries were determined in $[1,38]$.

We will first constrain the CFDs by fitting the flavor symmetries of the descendant CFDs with the known ones after decoupling the fundamental flavors. Comparing with the 
flavor symmetry enhancements predicted in [21], we find that the following graph should be a subgraph of the actual marginal CFD:

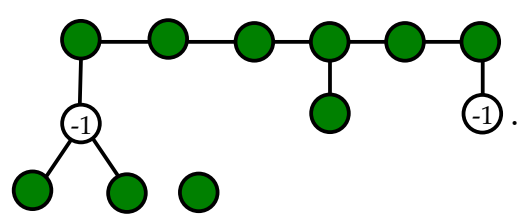

The CFD tree generated by decoupling fundamental flavors is shown in figure 18 .

To see that this is the only way the $\mathrm{SU}(2)$ nodes can attach, consider the first descendant. From the enhancements of the flavor symmetry [21] there are two enhancement patterns:

$$
\begin{array}{ll}
\mathrm{SU}(2 n)_{0}+2 \boldsymbol{A} \boldsymbol{S}+6 \boldsymbol{F}: & G_{F}=E_{6} \times \mathrm{SU}(2) \times \mathrm{U}(1)^{2} \\
\mathrm{SU}(2 n)_{1}+2 \boldsymbol{A} \boldsymbol{S}+6 \boldsymbol{F}: & G_{F}=\mathrm{SO}(12) \times \mathrm{SU}(2)^{3} .
\end{array}
$$

To get the first, it is clear that two $\mathrm{SU}(2)$ nodes have to attached with a single ( -1$)$ vertex to the longer tail of $E_{7}$. From the latter it is clear that another $(-1)$ vertex has to be attached to the short tail of the $E_{7}$ diagram. Note that decoupling only the fundamental matter retains an $\mathrm{SU}(2)$ factor in the flavor symmetry. We will see that additional constraints on this node follow from the decoupling of the anti-symmetric matter. The subtree that corresponds to the models with a fixed number of $2 \boldsymbol{A S}$ and decoupling $\boldsymbol{F}$ is consistent indeed with all the known flavor symmetry enhancements.

On the other hand, if we decouple the anti-symmetric hypermultiplets, we obtain the following gauge theories:

$$
\mathrm{SU}(2 n)_{0}+2 \boldsymbol{A} \boldsymbol{S}+8 \boldsymbol{F} \rightarrow \mathrm{SU}(2 n)_{ \pm(n-2)}+1 \boldsymbol{A} \boldsymbol{S}+8 \boldsymbol{F} \rightarrow\left\{\begin{array}{l}
\mathrm{SU}(2 n)_{2 n-4}+0 \boldsymbol{A} \boldsymbol{S}+8 \boldsymbol{F} \\
\mathrm{SU}(2 n)_{0}+0 \boldsymbol{A} \boldsymbol{S}+8 \boldsymbol{F} \\
\mathrm{SU}(2 n)_{-2 n+4}+0 \boldsymbol{A} \boldsymbol{S}+8 \boldsymbol{F}
\end{array}\right.
$$

Further decoupling a fundamental flavor, we should get:

$$
\begin{aligned}
\mathrm{SU}(2 n)_{2 n-4}+8 \boldsymbol{F} & \rightarrow \mathrm{SU}(2 n)_{2 n-4 \pm 1 / 2}+7 \boldsymbol{F} \\
\mathrm{SU}(2 n)_{0}+8 \boldsymbol{F} & \rightarrow \mathrm{SU}(2 n)_{ \pm 1 / 2}+7 \boldsymbol{F} \\
\mathrm{SU}(2 n)_{-2 n+4}+8 \boldsymbol{F} & \rightarrow \mathrm{SU}(2 n)_{-2 n+4 \pm 1 / 2}+7 \boldsymbol{F}
\end{aligned}
$$

According to [1,38], the flavor symmetry enhancements for the theories without $\boldsymbol{A} \boldsymbol{S}$ are

$$
\begin{aligned}
\mathrm{SU}(2 n)_{ \pm(2 n-4)}+8 \boldsymbol{F}: & G_{\mathrm{F}}=\mathrm{U}(8) \times \mathrm{SU}(2) \\
\mathrm{SU}(2 n)_{0}+8 \boldsymbol{F}: & G_{\mathrm{F}}=\mathrm{U}(8) \times \mathrm{U}(1) \\
\mathrm{SU}(2 n)_{ \pm\left(2 n-4+\frac{1}{2}\right)}+7 \boldsymbol{F}: & G_{\mathrm{F}}=\mathrm{U}(7) \times \mathrm{SU}(2) \\
\mathrm{SU}(2 n)_{ \pm\left(2 n-4-\frac{1}{2}\right)}+7 \boldsymbol{F}: & G_{\mathrm{F}}=\mathrm{U}(7) \times \mathrm{U}(1) \\
\mathrm{SU}(2 n)_{ \pm \frac{1}{2}}+7 \boldsymbol{F}: & G_{\mathrm{F}}=\mathrm{U}(7) \times \mathrm{U}(1)
\end{aligned}
$$




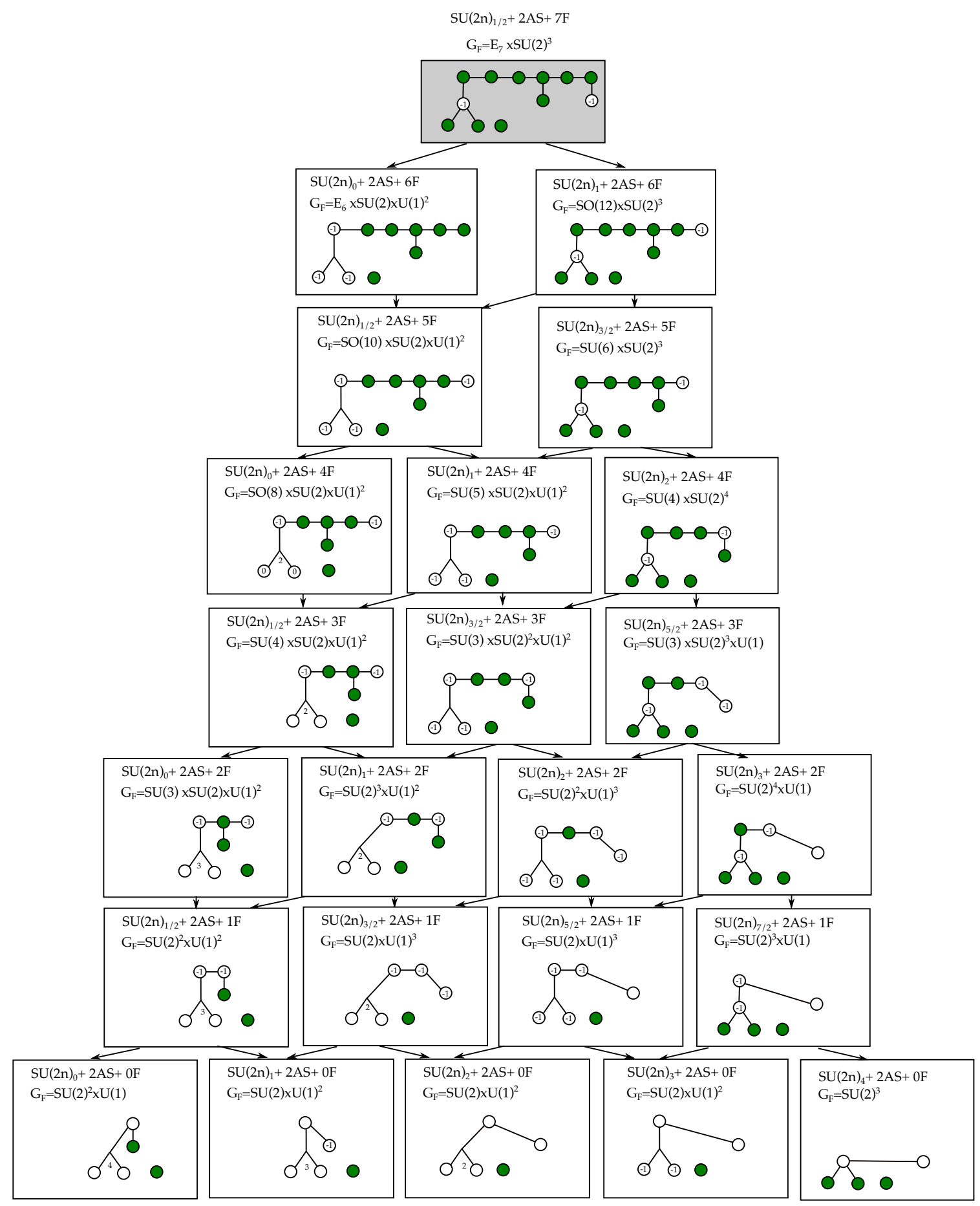

Figure 18. CFD-tree consistent with the decoupling of fundamental flavors of $\mathrm{SU}(2 n)_{0}+2 \boldsymbol{A} \boldsymbol{S}+8 \boldsymbol{F}$. The gauge theory descriptions and superconformal flavor symmetry $G_{\mathrm{F}}$ are labeled. 
With $1 \boldsymbol{A S}$, the superconformal flavor symmetries are [33]

$$
\begin{aligned}
\mathrm{SU}(2 n)_{ \pm(n-2)}+1 \boldsymbol{A} \boldsymbol{S}+8 \boldsymbol{F}: & G_{\mathrm{F}}=\mathrm{U}(8) \times \mathrm{SU}(2) \times \mathrm{U}(1) \\
\mathrm{SU}(2 n)_{0}+1 \boldsymbol{A} \boldsymbol{S}+8 \boldsymbol{F}: & G_{\mathrm{F}}=\mathrm{U}(8) \times \mathrm{U}(1) \times \mathrm{U}(1) \\
\mathrm{SU}(2 n)_{ \pm\left(n-2+\frac{1}{2}\right)}+1 \boldsymbol{A} \boldsymbol{S}+7 \boldsymbol{F}: & G_{\mathrm{F}}=\mathrm{U}(7) \times \mathrm{SU}(2) \times \mathrm{U}(1) \\
\mathrm{SU}(2 n)_{ \pm\left(n-2-\frac{1}{2}\right)}+1 \boldsymbol{A} \boldsymbol{S}+7 \boldsymbol{F}: & G_{\mathrm{F}}=\mathrm{U}(7) \times \mathrm{U}(1) \times \mathrm{U}(1) \\
\mathrm{SU}(2 n)_{ \pm \frac{1}{2}}+1 \boldsymbol{A} \boldsymbol{S}+7 \boldsymbol{F}: & G_{\mathrm{F}}=\mathrm{U}(7) \times \mathrm{U}(1) \times \mathrm{U}(1)
\end{aligned}
$$

Besides the consistency requirements with the known flavor symmetry enhancements, the other constraints for this marginal CFD are:

- Embedding of the BG-CFDs for the classical global symmetry $\mathrm{U}(8) \times \mathrm{U}(2) \times \mathrm{U}(1)_{T}$.

- The marginal CFD contains the Dynkin diagrams for $\widehat{E}_{7} \times \widehat{\mathrm{SU}(2)}^{2} \times \mathrm{SU}(2)$ that was observed in [9].

- Mass deforming the marginal CFD (i.e., CFD-transitioning on any of the -1-vertices) results in marked subdiagram that is a collection of non-affine Dynkin diagrams.

Finally, the resulting marginal CFD is highly constrained to be the following

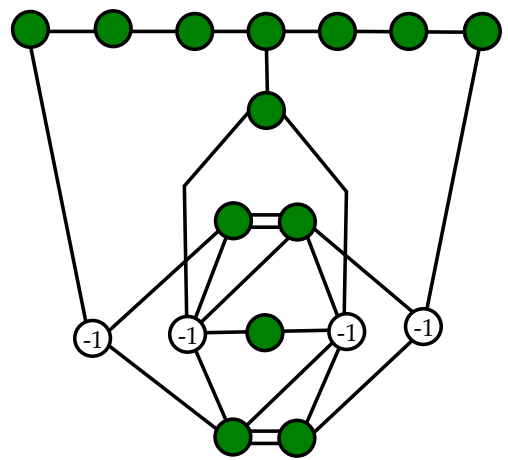

There are four $(-1)$-vertices in this graph. From left to the right, removing these vertices will correspond to the following four different ways of decoupling matter fields:

1. Decoupling $1 \boldsymbol{F}$, shifting the CS level $k$ by $\frac{1}{2}$.

2. Decoupling $1 \boldsymbol{A} \boldsymbol{S}$, shifting the CS level $k$ by $(n-2)$.

3. Decoupling $1 \boldsymbol{A} \boldsymbol{S}$, shifting the CS level $k$ by $-(n-2)$.

4. Decoupling $1 \boldsymbol{F}$, shifting the CS level $k$ by $-\frac{1}{2}$.

We list all the possible maximal embedding of BG-CFDs into this marginal CFD in figure 20. Together with the classical flavor symmetry shown in table 2, this put constraints on the possible quiver gauge theory descriptions. We will return to this and the resulting dualities in section 6 .

From this proposed marginal CFD, we obtain the tree of descendants, part of which is shown in figure 19. The sub-tree with gauge theory descriptions $\mathrm{SU}(2 n)_{k}+1 \boldsymbol{A} \boldsymbol{S}+m \boldsymbol{F}$ is shown in figure 21. Besides the $\mathrm{SU}(2 n)$ gauge theory descriptions, in figure 19 there is already a descendant theory with $G_{\mathrm{F}}=\mathrm{U}(4)^{2} \times \mathrm{SU}(2)$ with a different quiver gauge theory description. 


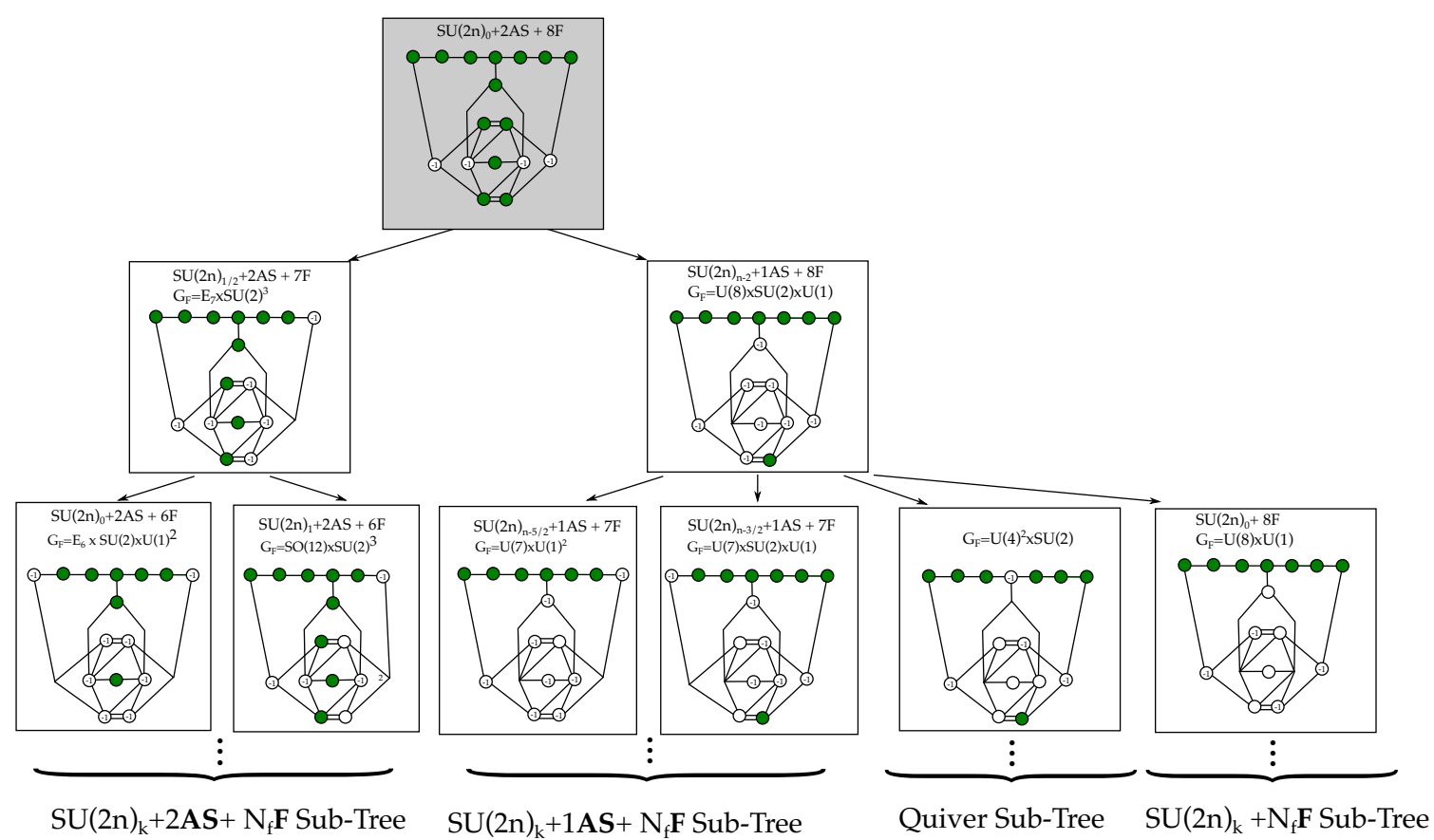

Figure 19. CFD-tree consistent with the decoupling of antisymmetric flavors of $\mathrm{SU}(2 n)_{0}+2 \boldsymbol{A} \boldsymbol{S}+$ $8 \boldsymbol{F}$. On the l.h.s. , the decoupling of fundamental flavors results in the sub-tree shown in figure 18. The mass deformations removing the anti-symmetric representations is consistent with the enhancement of flavor symmetries. The models with $1 \boldsymbol{A S}$ are consistent with the gauge theory description in tables 8 and 11 .

\section{$5.3 \mathrm{SU}(2 n+1)_{0}+2 A S+8 F$}

We can apply similar logic to the case with $\mathrm{SU}(N=2 n+1)_{0}$ gauge group and $2 \boldsymbol{A} \boldsymbol{S}+8 \boldsymbol{F}$. Note that in this case the marginal theory in $5 \mathrm{~d}$ has flavor symmetry [9]

$$
G_{\mathrm{F}}^{\text {marginal }}=\widehat{\mathrm{SO}(16)} \times \widehat{\mathrm{SU}(2)} \times \mathrm{SU}(2) .
$$

Using the known UV flavor symmetry enhancements as before, we conjecture the marginal CFD for this class of models to be

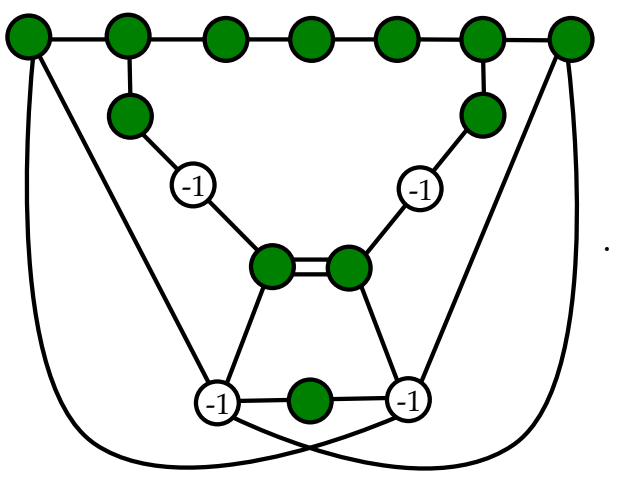

It contains the BG-CFDs for the classical flavor symmetries $\mathrm{U}(8)$ and $\mathrm{U}(2)$, and furthermore the marked vertices in the CFD realize $G_{\mathrm{F}}^{\text {marginal }}$. There are four $(-1)$-vertices in this graph. From upper left to the bottom right, removing these vertices will correspond to: 


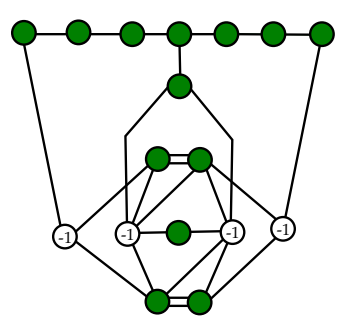

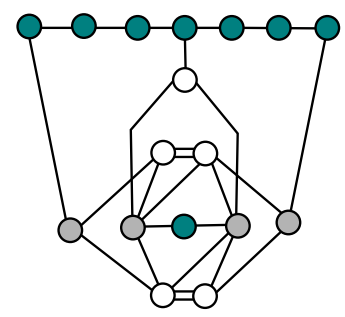

$\mathrm{U}(8) \times \mathrm{U}(2)$

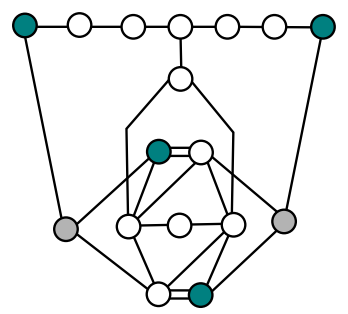

$\mathrm{SO}(4) \times \mathrm{SO}(4)$

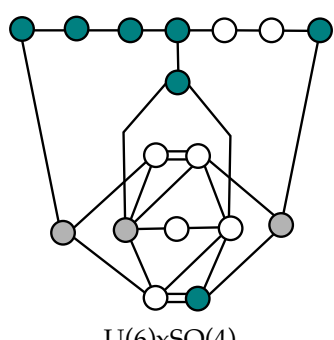

$\mathrm{U}(6) \times \mathrm{SO}(4)$

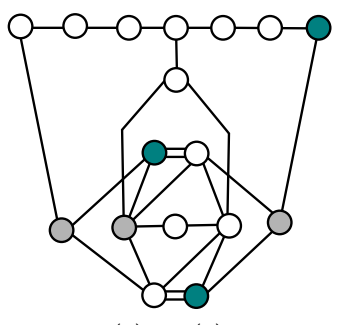

$\mathrm{U}(2) \times \mathrm{SO}(4)$
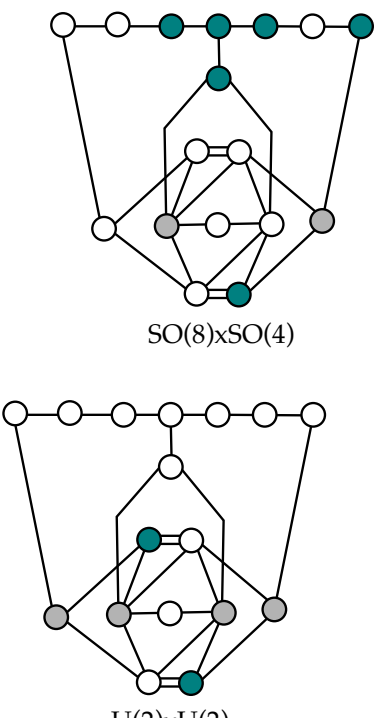

$\mathrm{U}(2) \times \mathrm{U}(2)$
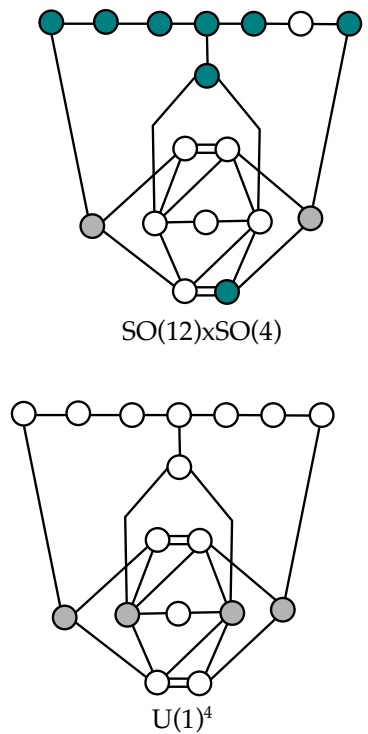

Figure 20. At the top we show the CFD for the marginal theory $\mathrm{SU}(2 n)+2 \boldsymbol{A} \boldsymbol{S}+8 \boldsymbol{F}$, and below, using the notation of section 4 the possible embeddings of the BG-CFDs into the marginal CFD. Below the diagrams we note the classical flavor symmetry of the corresponding weakly coupled description.

1. Decoupling $1 \boldsymbol{F}$, shifting the CS level $k$ by $\frac{1}{2}$.

2. Decoupling $1 \boldsymbol{F}$, shifting the CS level $k$ by $-\frac{1}{2}$.

3. Decoupling $1 \boldsymbol{A S}$, shifting the CS level $k$ by $\left(n-\frac{3}{2}\right)$.

4. Decoupling $1 \boldsymbol{A S}$, shifting the CS level $k$ by $-\left(n-\frac{3}{2}\right)$.

The descendants are in agreement with the $2 \boldsymbol{A} \boldsymbol{S}+N_{f} \boldsymbol{F}$ flavor symmetry enhancements in [21] and the ones with one $\boldsymbol{A} \boldsymbol{S}$ (as listed in appendix B) and no $\boldsymbol{A} \boldsymbol{S}$ in [1,33, 38]. The CFD-descendants that model the theories with 1 or $2 \boldsymbol{A S}$ are shown in figure 22. Note that there are more descendants, which will correspond to other gauge theory descriptions. The possible BG-CFD embeddings, are shown in figure 23, from which we can determine alternative weakly coupled descriptions, which in particular should model some of the other descendants. 


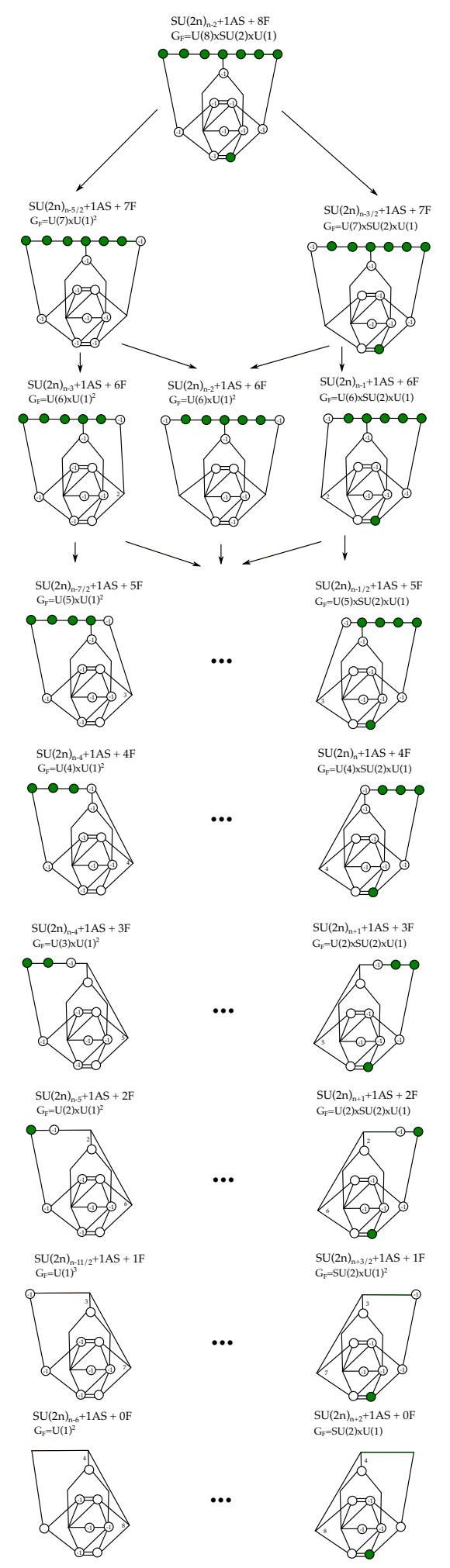

Figure 21. Sub-trees of the CFD-tree figure 19: $\mathrm{SU}(2 n)_{0}+1 \boldsymbol{A} \boldsymbol{S}+N_{f} \boldsymbol{F}, N_{f} \leq 7$ decoupling the fundamental flavors results in the sub-tree shown in this figure. The enhancements are consistent with the ones. 


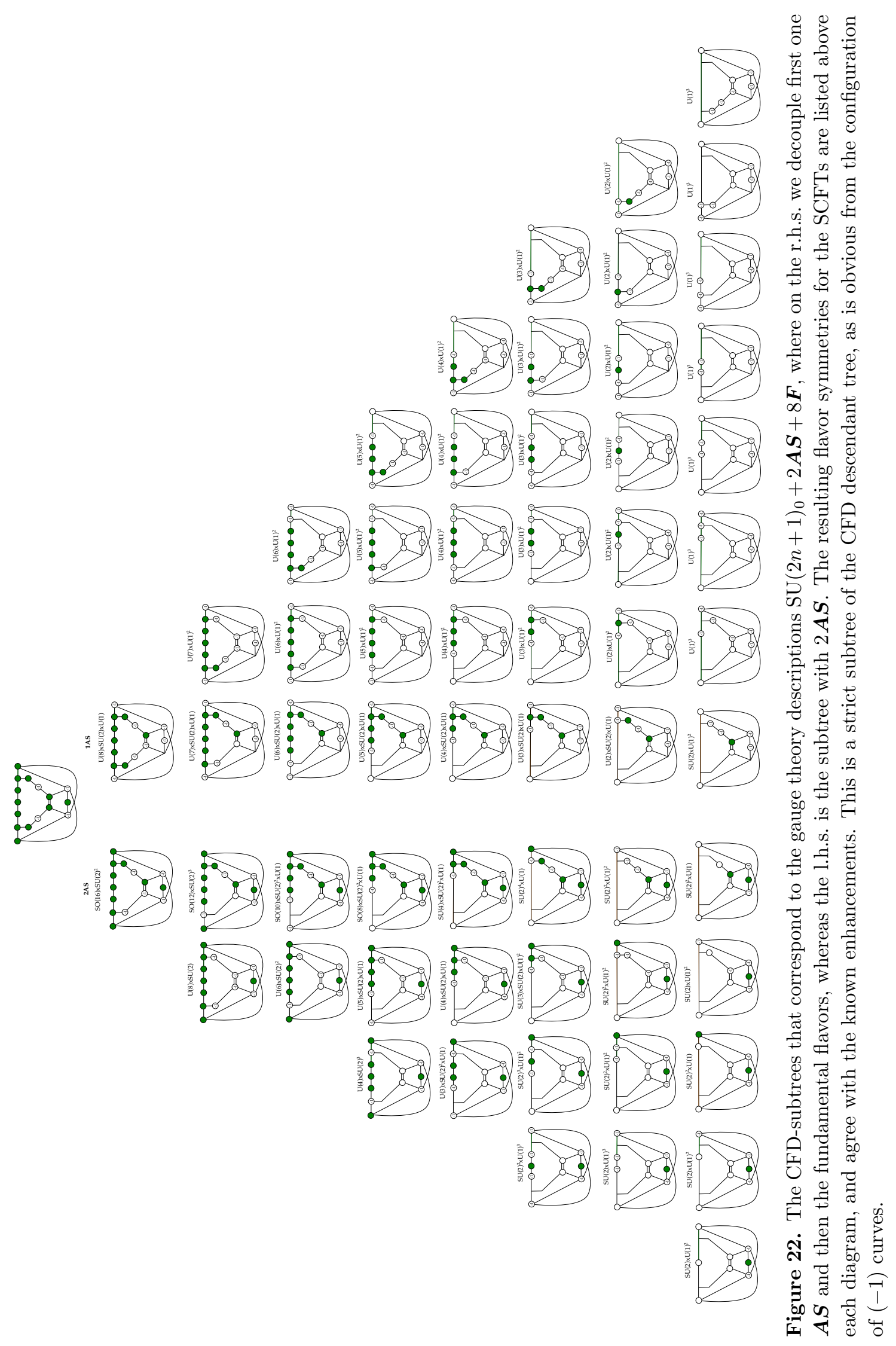



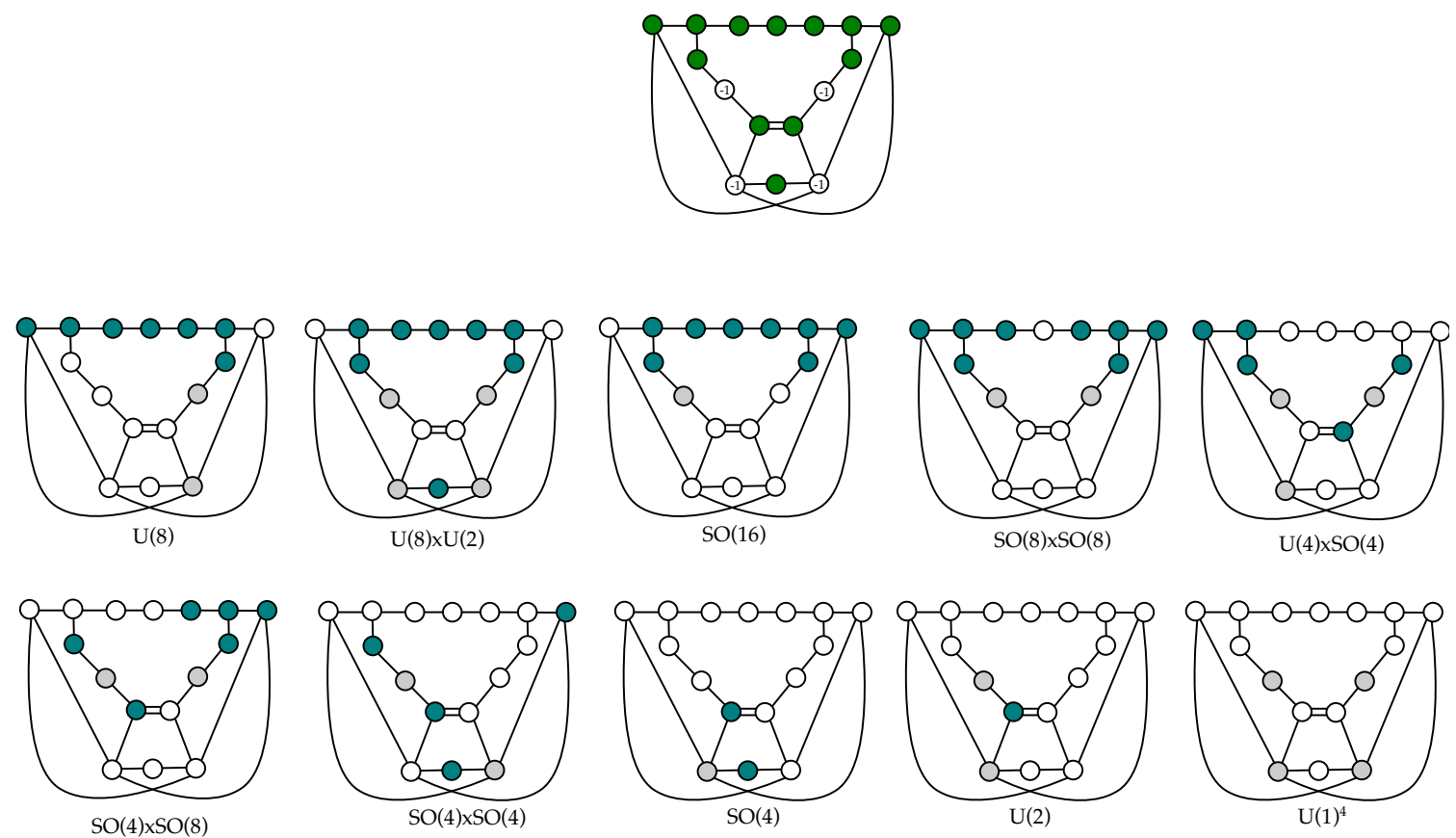

Figure 23. The possible embeddings of the BG-CFDs into the marginal CFD, which is shown at the top, for $\mathrm{SU}(2 n+1)+2 \boldsymbol{A} \boldsymbol{S}+8 \boldsymbol{F}$. Below the diagrams we note the classical flavor symmetry for the putative weakly coupled description.

\section{$5.4 \mathrm{SU}(4)_{0}+2 A S+8 F$ and $\left(E_{7}, \mathrm{SO}(7)\right)$ conformal matter}

A special case of the theories discussed in section 5.2 is $n=2: \mathrm{SU}(4)_{0}+2 \boldsymbol{A S}+8 \boldsymbol{F}$. In this case there is an enhanced superconformal symmetry $G_{\mathrm{F}}^{\text {marginal }}=\widehat{E}_{7} \times \widehat{\mathrm{SO}(7)}$ for the marginal theory, which has its origin in the $6 \mathrm{~d}$ realization in terms of the $\left(E_{7}, \mathrm{SO}(7)\right)$ minimal conformal matter.

The marginal CFD can in this case in fact be computed directly using a geometric resolution from the conformal matter description, which is done in appendix C.3. From this we determine the marginal CFD to be:

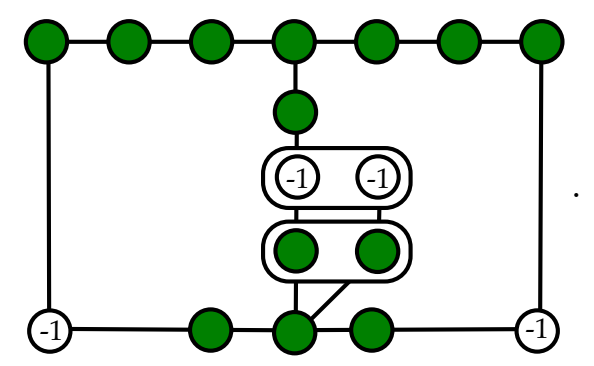

Note that the in the middle of the graph, there is a reducible vertex containing two $(-1)$-vertices, which are encircle to indicate that they are homologous, and that they have to be removed simultaneously. ${ }^{16}$ Likewise, the reducible vertex below it contains two

\footnotetext{
${ }^{16}$ In the resolved Calabi-Yau threefold geometry, they correspond to two curves with normal bundle $\mathcal{O}(-1)+\mathcal{O}(-1)$ that are homologous in the Calabi-Yau threefold but not on the surface components.
} 
$(-2)$-vertices that are encircled and homologous curves in the geometry, and are part of the $\mathrm{SO}(7)$ affine Dynkin diagram.

This graph indeed contains the BG-CFDs for $8 \boldsymbol{F}$ with classical flavor symmetry U(8) and $2 \boldsymbol{A} \boldsymbol{S}$ with classical flavor symmetry $\operatorname{Sp}(2)$, see table 2 :

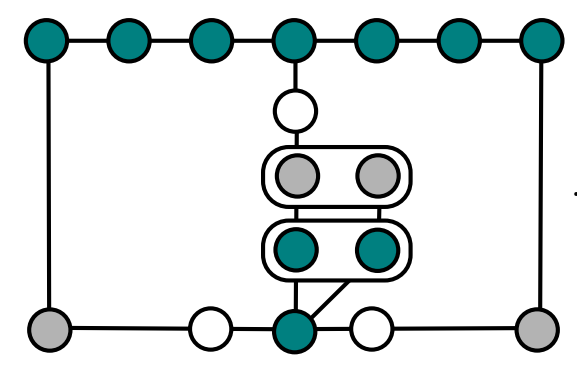

Thus if we were to bootstrap this theory as we did in the general case of $\mathrm{SU}(2 n)$, we would arrive at precisely this diagram. Consistency with the known flavor symmetry enhancements can also be checked.

On the other hand, there is another way of embedding the BG-CFD with classical flavor symmetry $\mathrm{SO}(12)$ and $\mathrm{SO}(4)$ :

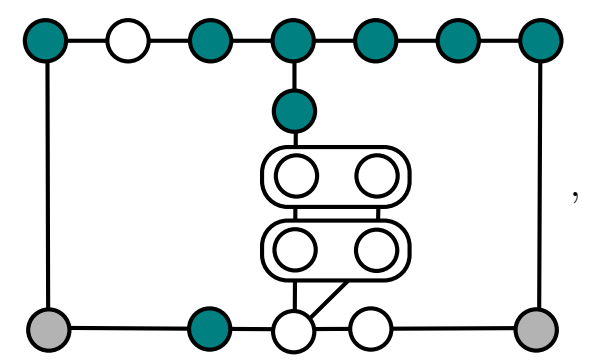

which corresponds to the quiver gauge theory $6 \boldsymbol{F}-\operatorname{Sp}(2)-\operatorname{Sp}(1)-2 \boldsymbol{F}$, see section 6 for more details.

Some of the descendants of the $\left(E_{7}, \mathrm{SO}(7)\right)$ marginal CFD are shown in figure 24 . For example, after removing the reducible vertex containing the two $(-1)$-vertices, we arrive at the theory (3), where the reducible vertex below it will contain two $(-1)$-vertices and the vertex above it will become a $(-1)$-vertex. Then if we remove the reducible vertex containing two (-1)-vertices in the graph (3) to get graph (8), the reducible vertex below it will become a 0 -vertex because of the double connection in (3).

We list the $\mathrm{SU}(4)$ gauge theory descriptions of these theories in table 3, which can be compared with the flavor symmetry enhancements in the literature [21,33]. For the CFDs (2), (4) and (6) with $2 \boldsymbol{A} \boldsymbol{S}$, the enhanced flavor symmetry matches the table 3 in [21]. For the cases (3) and (7) with $1 \boldsymbol{A S}$, the flavor symmetry was correctly predicted in [33]. For the case 8 , which does not have any anti-symmetric matter, the CFD matches the descendant from $\left(D_{6}, D_{6}\right)$ marginal CFD [1] with flavor symmetry $\mathrm{SU}(8) \times \mathrm{SU}(2) \times \mathrm{SU}(2)$. Especially, there should not be any additional extremal $(-1)$-curve in the middle part of the picture. Finally, for the case (5), the CFD transition from case (2) does not correspond to the decoupling of a matter multiplet in the SU(4) gauge theory. Hence this theory with $G_{\mathrm{F}}=E_{7} \times \mathrm{SU}(2) \times \mathrm{SU}(2)$ is not expected to have an $\mathrm{SU}(4)$ gauge theory description. On 
(1)
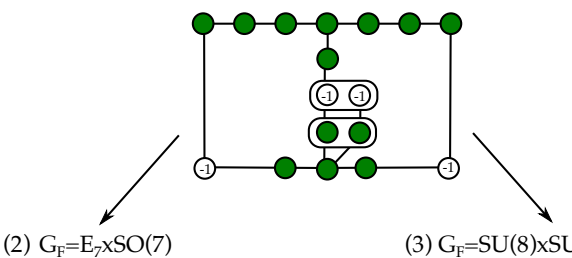

(2) $\mathrm{G}_{\mathrm{F}}=\mathrm{E}_{7} \mathrm{xSO}(7)$

(3) $\mathrm{G}_{\mathrm{F}}=\mathrm{SU}(8) \times \mathrm{SU}(4)$
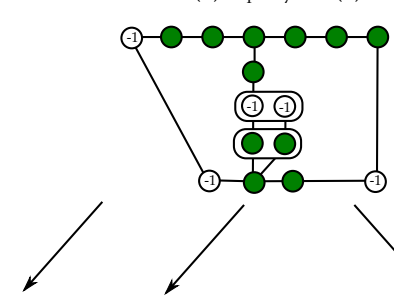

$0-0-0-0$

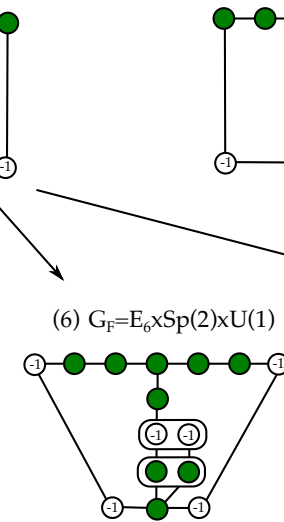

(-1) (1)

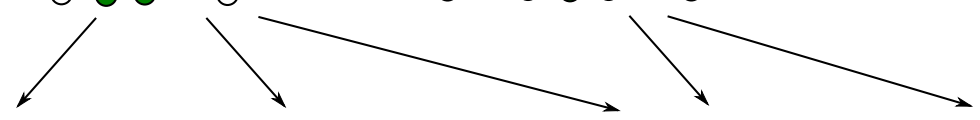

(4) $\mathrm{G}_{\mathrm{F}}=\mathrm{SO}(12) \times \mathrm{SO}(7)$

(5) $\mathrm{G}_{\mathrm{F}}=\mathrm{E}_{7} \times \mathrm{SO}(4)$

(7) $\mathrm{G}_{\mathrm{F}}=\mathrm{SU}(7) \times \mathrm{SU}(3) \times \mathrm{U}(1)$

(8) $\mathrm{G}_{\mathrm{F}}=\mathrm{SU}(8) \times \mathrm{SO}(4)$
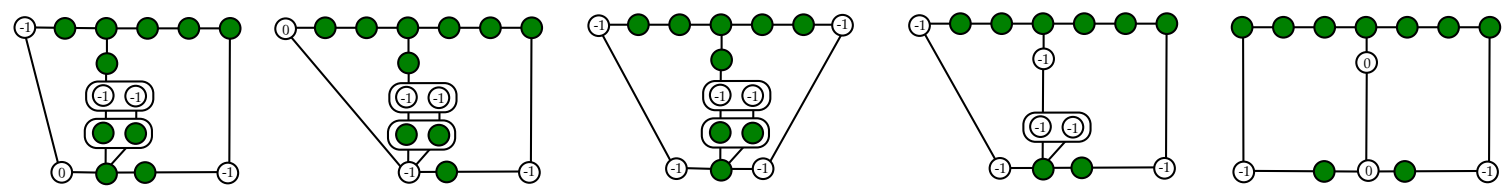

Figure 24. $\left(E_{7}, \mathrm{SO}(7)\right)$ : the figure shows the first few descendants of the marginal CFD (5.13) for the $\left(E_{7}, \mathrm{SO}(7)\right)$ theory, including the enhanced superconformal flavor symmetries $G_{\mathrm{F}}$.

\begin{tabular}{|c|c|c|c|}
\hline CFD No. & Matter fields & $\kappa$ & $G_{\mathrm{F}}$ \\
\hline$(1)$ & $2 \boldsymbol{A} \boldsymbol{S}+8 \boldsymbol{F}$ & 0 & - \\
$(2)$ & $2 \boldsymbol{A} \boldsymbol{S}+7 \boldsymbol{F}$ & $1 / 2$ & $E_{7} \times \mathrm{SO}(7)$ \\
$(3)$ & $1 \boldsymbol{A} \boldsymbol{S}+8 \boldsymbol{F}$ & 0 & $\mathrm{SU}(4) \times \mathrm{SU}(8)$ \\
$(4)$ & $2 \boldsymbol{A} \boldsymbol{S}+6 \boldsymbol{F}$ & 1 & $\mathrm{SO}(7) \times \mathrm{SO}(12)$ \\
$(5)$ & - & - & $E_{7} \times \mathrm{SU}(2) \times \mathrm{SU}(2)$ \\
$(6)$ & $2 \boldsymbol{A S}+6 \boldsymbol{F}$ & 0 & $\mathrm{Sp}(2) \times E_{6} \times \mathrm{U}(1)$ \\
$(7)$ & $1 \boldsymbol{A S}+7 \boldsymbol{F}$ & $1 / 2$ & $\mathrm{SU}(7) \times \mathrm{SU}(3) \times \mathrm{U}(1)$ \\
$(8)$ & $8 \boldsymbol{F}$ & 0 & $\mathrm{SU}(8) \times \mathrm{SU}(2) \times \mathrm{SU}(2)$ \\
\hline
\end{tabular}

Table 3. The $\mathrm{SU}(4)_{\kappa}$ gauge theory description and superconformal flavor symmetry $G_{\mathrm{F}}$ of the descendant theories from the $\left(E_{7}, \mathrm{SO}(7)\right)$ marginal CFD. The CFD numbering corresponds to that in figure 24. Model (5) does not have a description in terms of an $\mathrm{SU}(4)$ gauge theory, but it has a $6 \boldsymbol{F}-\mathrm{Sp}(2)-\mathrm{Sp}(1)$ quiver gauge theory description instead.

the other hand, from the quiver gauge theory $6 \boldsymbol{F}-\mathrm{Sp}(2)-\mathrm{Sp}(1)-2 \boldsymbol{F}$ of the marginal theory, the CFD (5) is generated by decoupling the $2 \boldsymbol{F}$ of the $\operatorname{Sp}(1)$ gauge group. Hence we expect this theory to have a $6 \boldsymbol{F}-\mathrm{Sp}(2)-\mathrm{Sp}(1)$ quiver gauge theory description. 


\section{Descendants and dualities}

A 5d SCFT can be effectively described by multiple gauge theories at low-energy, equivalently different gauge theories can have the same UV-fixed point. Such theories can be viewed as dual effective descriptions of the same UV 5d SCFT. In particular, because of the IR effective nature of $5 \mathrm{~d}$ gauge theories, these are called UV-dualities.

In this section we study UV-dualities among quiver gauge theories from the point of view of the CFDs. More precisely, by embedding the BG-CFDs into the marginal CFDs we are able to predict possible dual gauge theory phases, which UV-complete to a 6d SCFT. We then check if these gauge theories are consistent geometrically, by studying the ruling of the resolutions corresponding to different descendants. From these resolutions, we also consistently blow up the collection of surfaces to get a candidate resolved geometry for the marginal theory. We then compute the triple intersection numbers and check them against the prepotential computed from the gauge theory.

Having set the dual effective descriptions for the marginal theory we can immediately predict many novel dualities for the descendant theories. In many cases, we support these by explicitly finding the corresponding rulings in the resolved Calabi-Yau threefolds engineering the $5 \mathrm{~d}$ theories. In addition, we also compute the prepotential of the candidate duals. For many theories, which are not distinguished by non-trivial physical theta angles, we check that the prepotentials match in some region of the Coulomb branches. This, together with the prediction of the superconformal flavor symmetries supplied by the CFD and BG-CFD embeddings, provides a good test for these novel proposed UV-dualities.

\subsection{Descendants and dualities for minimal $\left(E_{6}, E_{6}\right)$ conformal matter}

As we showed in [2], the known weakly coupled quiver description of the $\left(E_{6}, E_{6}\right)$ minimal conformal matter theory only captures a very small subset of descendant SCFTs in 5d. Starting with the CFD, we have seen that there are multiple ways that BG-CFDs can be embedded. This results in new dualities, both for the marginal theory as well as the descendants. In this class of theories, all dualities can be checked by comparing with the geometry and finding the corresponding "dual" rulings of the surfaces.

\subsubsection{An asymmetric quiver}

In this section we will consider quiver gauge theories that are descendants of the quiver

$$
6 \boldsymbol{F}-\mathrm{SU}(4)_{0}-\mathrm{Sp}(1)_{0}-\mathrm{Sp}(1)-2 \boldsymbol{F} .
$$

This quiver is derived from geometric considerations, in appendix C.2 and shown to be marginal, and to have as $6 \mathrm{~d} \mathrm{UV}$ fixed point the minimal $\left(E_{6}, E_{6}\right)$ conformal matter theory. While the $\theta$-angle of the central $\mathrm{Sp}(1)$ gauge node is not directly fixed by the geometry, ${ }^{17}$ the superconformal flavor symmetries for the alternate case where $\theta=\pi$ are not consistent

\footnotetext{
${ }^{17}$ If we assume the following empirical evidence that a trivial theta angle leads to an enhancement of flavor symmetry at strong coupling, whereas a non-trivial one leads to an abelian factor, we can actually present a criterion to compute the theta angle at least for a $\mathrm{SU}(2)$ gauge theory factor: if the surface is ruled over a curve corresponding to a green node in the CFD, then $\theta=0$, otherwise $\theta=\pi$.
} 
with the quiver being a descendant of $\left(E_{6}, E_{6}\right)$ conformal matter [70], and thus we are led inexorably to the conclusion that the only option is $\theta=0$.

The descendants of this quiver have the form

$$
\left(m_{1}, k, m_{2}\right): \quad m_{1} \boldsymbol{F}-\mathrm{SU}(4)_{k}-\mathrm{Sp}(1)_{0}-\mathrm{Sp}(1)-m_{2} \boldsymbol{F} .
$$

If $m_{2}=0$ then we must specify a $\theta$-angle for the rightmost $\operatorname{Sp}(1)$ factor, we shall, without ambiguity, use the shorthand $m_{2}=0, \pi$ for these two options.

The classical flavor group of the marginal theory is $\mathrm{U}(6) \times \mathrm{SO}(4) \times \mathrm{SU}(2) \times \mathrm{U}(1)^{4}$, where the first two factors are the global symmetry groups rotating the hypermultiplets on each of the two flavor nodes. The BG-CFDs associated to these two flavor groups must then be embedded into the marginal $\left(E_{6}, E_{6}\right) \mathrm{CFD}$, which was given in (4.34). Up to the symmetry of the CFD there is a unique form of such an embedding, which is

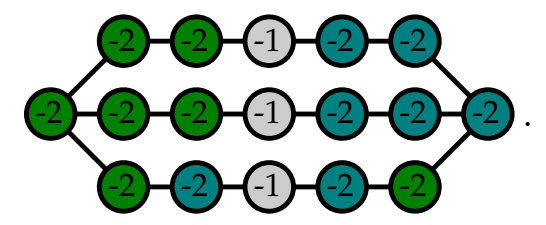

Quivers of the form (6.2) admit dualities amongst theories with different $\left(m_{1}, k, m_{2}\right)$, and these can be observed from the symmetry of the descandent CFDs. Let us consider the first descendant of the marginal CFD as an illustrative example; despite the fact that there are three (-1)-curves, each of which gives a CFD-transition to a descendant CFD, all of these three descendants are the same CFD up to a reordering in the 2d-plane in which we draw the image. However if we consider the CFD together with the marked BG-CFDs, as in (6.3), then there would appear to be three distinct gauge theory descendants, for which the embeddings of the BG-CFDs are
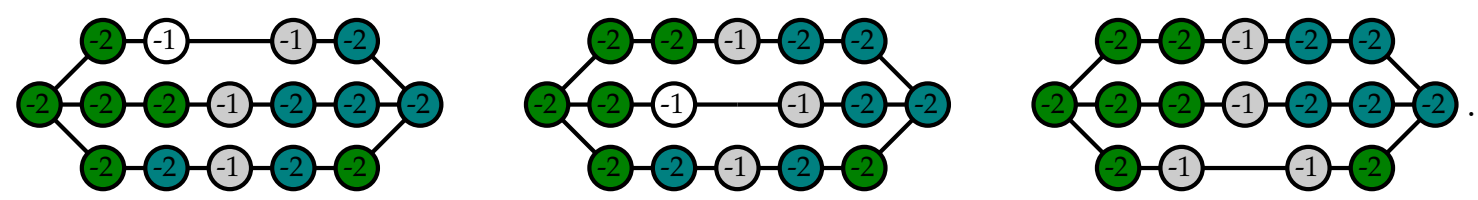

These correspond to the three gauge theory descriptions

$$
\left(m_{1}, k, m_{2}\right)=(5,+1 / 2,2),(5,-1 / 2,2),(6,0,1) .
$$

Since the underlying CFD for each of these theories is the same then these theories have the same interacting SCFT as their UV fixed point, and as such they are all dual to each other. To be extremely explicit, the CFD implies that the three quiver gauge theories

$$
\begin{aligned}
& 5 \boldsymbol{F}-\mathrm{SU}(4)_{1 / 2}-\mathrm{Sp}(1)_{0}-\mathrm{Sp}(1)-2 \boldsymbol{F} \\
& 5 \boldsymbol{F}-\mathrm{SU}(4)_{-1 / 2}-\mathrm{Sp}(1)_{0}-\mathrm{Sp}(1)-2 \boldsymbol{F} \\
& 6 \boldsymbol{F}-\mathrm{SU}(4)_{0}-\mathrm{Sp}(1)_{0}-\mathrm{Sp}(1)-1 \boldsymbol{F},
\end{aligned}
$$

flow to the same $5 \mathrm{~d} \mathcal{N}=1 \mathrm{SCFT}$ in the UV. In this way one can see that novel dualities between quiver gauge theories, with the same gauge algebra, can be observed from the CFD. 
We determine the superconformal flavor symmetry for every descendant of the quiver (6.1), and furthermore we determine for what values of $\left(m_{1}, k, m_{2}\right)$ the descendant quivers (6.2) are dual.

More generally, if we compute the prepotential for the two sets of quiver specified by $\left(m_{1}, k, m_{2}\right)$ and $\left(\widetilde{m}_{1}, \widetilde{k}, \widetilde{m}_{2}\right)$, it matches in a non-trivial region of the two gauge theories respectively, if the following conditions are satisfied,

$$
\widetilde{m}_{1}=1-k+m_{2}+\frac{m_{1}}{2}, \quad \widetilde{m}_{2}=\frac{1}{2}\left(m_{1}+2 k-2\right) \quad \widetilde{k}=\frac{1}{4}\left|m_{1}-2 m_{2}-2-2 k\right|
$$

Since the prepotenial analysis is not sensitive to the $\theta$-angles these expressions involve some care when $m_{2}=0, \pi$. For $m_{2}=0$ they capture the dualities as determined from the CFD. These results for the descendants of the marginal quiver with $\left(m_{1}, k, m_{2}\right)=(6,0,2)$ are summarized in table 4 .

\subsubsection{Maximal quivers}

As a proof of principle we present here a prospective dual marginal description of $\left(E_{6}, E_{6}\right)$ conformal matter on a circle and its descendants, which satisfy the classical flavor symmetry embedding in the CFD diagrams in figure 15. This IR effective description was already introduced in 4.7, and it has ten full hypermultiplets, the maximal number possible. This feature makes this possible description very interesting, since it would provide the IR effective gauge theories for as many descendants as possible. It reads

$$
5 \boldsymbol{F}-\mathrm{Sp}(1)-\mathrm{SO}(6)-\mathrm{Sp}(1)-5 \boldsymbol{F} .
$$

While there is no evidence from geometry that this is indeed a quiver description of $\left(E_{6}, E_{6}\right)$ conformal matter, we will, in this section, determine the descendants and their superconformal flavor symmetries under the assumption that this quiver is indeed a realized description. It would be interesting to study the Coulomb branch metric and the BPS string tensions of the full quiver in order to verify the validity of this effective theory.

To express the global symmetries in a concise way we first introduce a helpful notation for the $E$-type exceptional simple groups

$$
E^{(k)}=\left\{\begin{array}{ll}
E_{k+1} & \text { for } k=0, \cdots, 7 \\
\widetilde{E}_{1}=\mathrm{U}(1) & \text { for } k=\pi
\end{array} .\right.
$$

The descendant quivers have the form

$$
m_{1} \boldsymbol{F}-\mathrm{Sp}(1)-\mathrm{SO}(6)-\mathrm{Sp}(1)-m_{2} \boldsymbol{F}
$$

where we again allow the abuse of notation to write $m_{1}, m_{2}=0, \pi$ to describe the $\theta$-angle when the respective flavor nodes become trivial. If we write the tuple $\left(m_{1}, m_{2}\right)$ to describe one of these descendant quivers we find, from an analysis of the CFD and its descendants, 


\begin{tabular}{|c|c|}
\hline$\left(m_{1}, k, m_{2}\right)$ & Superconformal Flavor Symmetry \\
\hline$(5, \pm 1 / 2,2),(6,0,1)$ & $E_{6} \times E_{6}$ \\
\hline$(4, \pm 1,2),(6,0,0),(6,0, \pi)$ & $E_{6} \times \mathrm{SU}(6)$ \\
\hline$(5, \pm 1 / 2,1),(4,0,2)$ & $\mathrm{SO}(10)^{2} \times \mathrm{U}(1)$ \\
\hline$(3, \pm 3 / 2,2)$ & $E_{6} \times \mathrm{SU}(3)^{2}$ \\
\hline$(3, \pm 1 / 2,2),(4, \pm 1,1),(5, \pm 1 / 2,0),(5, \pm 1 / 2, \pi)$ & $\mathrm{SO}(10) \times \mathrm{SU}(5) \times \mathrm{U}(1)$ \\
\hline$(4,0,1)$ & $\mathrm{SO}(8)^{2} \times \mathrm{U}(1)^{2}$ \\
\hline$(2, \pm 2,2)$ & $E_{6} \times \mathrm{SU}(2)^{2} \times \mathrm{U}(1)$ \\
\hline$(2, \pm 1,2),(3, \pm 3 / 2,1)$ & $\mathrm{SO}(10) \times \mathrm{SU}(3) \times \mathrm{SU}(2) \times \mathrm{U}(1)$ \\
\hline$(2,0,2),(4, \pm 1,0)$ & $\mathrm{SO}(10) \times \mathrm{SU}(4) \times \mathrm{U}(1)$ \\
\hline$(4, \pm 1, \pi)$ & $\mathrm{SU}(5)^{2} \times \mathrm{U}(1)$ \\
\hline$(3, \pm 1 / 2,1),(4,0,0),(4,0, \pi)$ & $\mathrm{SO}(8) \times \mathrm{SU}(4) \times \mathrm{U}(1)^{2}$ \\
\hline$(1, \pm 5 / 2,2)$ & $E_{6} \times \mathrm{SU}(2) \times \mathrm{U}(1)$ \\
\hline$(1, \pm 3 / 2,2),(2, \pm 2,1)$ & $\mathrm{SO}(10) \times \mathrm{SU}(2) \times \mathrm{U}(1)^{2}$ \\
\hline$(1, \pm 1 / 2,2)$ & $\mathrm{SO}(10) \times \mathrm{SU}(3) \times \mathrm{U}(1)$ \\
\hline$(2, \pm 1,1)$ & $\mathrm{SO}(8) \times \mathrm{SU}(2)^{2} \times \mathrm{U}(1)^{2}$ \\
\hline$(2,0,1),(3, \pm 1 / 2,0)$ & $\mathrm{SO}(8) \times \mathrm{SU}(3) \times \mathrm{U}(1)^{2}$ \\
\hline$(3, \pm 3 / 2,0)$ & $\mathrm{SO}(10) \times \mathrm{SU}(3) \times \mathrm{U}(1)$ \\
\hline$(3, \pm 3 / 2, \pi)$ & $\mathrm{SU}(5) \times \mathrm{SU}(3) \times \mathrm{SU}(2) \times \mathrm{U}(1)$ \\
\hline$(3, \pm 1 / 2, \pi)$ & $\mathrm{SU}(4)^{2} \times \mathrm{U}(1)^{2}$ \\
\hline$(0, \pm 3,2)$ & $E_{6} \times \mathrm{SU}(2)$ \\
\hline$(0, \pm 2,2)$ & $\mathrm{SO}(10) \times \mathrm{SU}(2) \times \mathrm{U}(1)$ \\
\hline$(1, \pm 5 / 2,1)$ & $\mathrm{SO}(10) \times \mathrm{U}(1)^{2}$ \\
\hline$(0, \pm 1,2),(2, \pm 2,0)$ & $\mathrm{SO}(10) \times \mathrm{SU}(2) \times \mathrm{U}(1)$ \\
\hline$(0, \pm 3 / 2,1)$ & $\mathrm{SO}(8) \times \mathrm{U}(1)^{3}$ \\
\hline$(2, \pm 2, \pi)$ & $\mathrm{SU}(5) \times \mathrm{SU}(2) \times \mathrm{U}(1)^{2}$ \\
\hline$(1, \pm 1 / 2,1),(2, \pm 1,0)$ & $\mathrm{SO}(8) \times \mathrm{SU}(2) \times \mathrm{U}(1)^{2}$ \\
\hline$(0,0,2)$ & $\mathrm{SO}(10) \times \mathrm{SU}(3)$ \\
\hline$(2, \pm 1, \pi)$ & $\mathrm{SU}(4) \times \mathrm{SU}(2)^{2} \times \mathrm{U}(1)^{2}$ \\
\hline$(2,0,0)$ & $\mathrm{SO}(8) \times \mathrm{SU}(2) \times \mathrm{U}(1)^{2}$ \\
\hline$(2,0, \pi)$ & $\mathrm{SU}(4) \times \mathrm{SU}(3) \times \mathrm{U}(1)^{2}$ \\
\hline$(0, \pm 3,1)$ & $\mathrm{SO}(10) \times \mathrm{U}(1)$ \\
\hline$(0, \pm 2,1)$ & $\mathrm{SO}(8) \times \mathrm{U}(1)^{2}$ \\
\hline$(0, \pm 1,1),(1, \pm 3 / 2,0)$ & $\mathrm{SO}(8) \times \mathrm{U}(1)^{2}$ \\
\hline$(0,0,1)$ & $\mathrm{SO}(8) \times \mathrm{SU}(2) \times \mathrm{U}(1)$ \\
\hline$(1, \pm 5 / 2,0)$ & $\mathrm{SO}(10) \times \mathrm{U}(1)$ \\
\hline$(1, \pm 5 / 2, \pi)$ & $\mathrm{SU}(5) \times \mathrm{U}(1)^{2}$ \\
\hline$(1, \pm 3 / 2, \pi)$ & $\mathrm{SU}(4) \times \mathrm{U}(1)^{3}$ \\
\hline$(1, \pm 1 / 2,0)$ & $\mathrm{SO}(8) \times \mathrm{U}(1)^{2}$ \\
\hline$(1, \pm 1 / 2, \pi)$ & $\mathrm{SU}(4) \times \mathrm{SU}(2) \times \mathrm{U}(1)^{2}$ \\
\hline$(0, \pm 3,0)$ & $\mathrm{SO}(10)$ \\
\hline$(0, \pm 2,0)$ & $\mathrm{SO}(8) \times \mathrm{U}(1)$ \\
\hline$(0, \pm 1,0)$ & $\mathrm{SO}(8) \times \mathrm{U}(1)$ \\
\hline$(0,0,0)$ & $\mathrm{SO}(8) \times \mathrm{U}(1)$ \\
\hline$(0, \pm 3, \pi)$ & $\mathrm{SU}(5) \times \mathrm{U}(1)$ \\
\hline$(0, \pm 2, \pi)$ & $\mathrm{SU}(4) \times \mathrm{U}(1)^{2}$ \\
\hline$(0, \pm 1, \pi)$ & $\mathrm{SU}(4) \times \mathrm{U}(1)^{2}$ \\
\hline$(0,0, \pi)$ & $\mathrm{SU}(4) \times \mathrm{SU}(2) \times \mathrm{U}(1)$ \\
\hline
\end{tabular}

Table 4. Dualities among and superconformal flavor symmetries for quivers of the form $m_{1} \boldsymbol{F}-$ $\mathrm{SU}(4)_{k}-\mathrm{Sp}(1)_{0}-\mathrm{Sp}(1)-m_{2} \boldsymbol{F}$ that are descendants of the quiver $6 \boldsymbol{F}-\mathrm{SU}(4)_{0}-\mathrm{Sp}(1)_{0}-\mathrm{Sp}(1)-2 \boldsymbol{F}$. We abuse notation and capture the $\theta$-angle of the rightmost $\operatorname{Sp}(1)$ when it has no fundamental hypermultiplets by allowing $m_{2}=0, \pi$. 
that the superconformal flavor symmetries are,

$$
\begin{array}{rll}
(4,5): & E_{6} \times E_{6} \\
(3,5): & \mathrm{SU}(6) \times E_{6} & \\
(2,5): & \mathrm{SU}(3)^{2} \times E_{6} & \\
(1,5): & \mathrm{SU}(2)^{2} \times \mathrm{U}(1) \times E_{6} & \\
(\theta, 5): & \mathrm{SU}(2) \times \mathrm{U}(1) \times E_{6} & \text { for } \theta=0, \pi \\
\left(m_{1}, m_{2}\right): & E^{\left(m_{1}\right)} \times E^{\left(m_{2}\right)} \times \mathrm{U}(1) & \text { for } m_{1}, m_{2}=0, \pi, 1,2,3,4 .
\end{array}
$$

\subsubsection{Dualities between different quivers}

We have two known quiver gauge theories that are marginal for the minimal $\left(E_{6}, E_{6}\right)$ conformal matter theory. These are

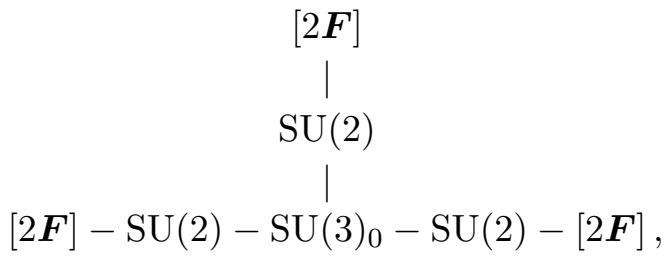

and

$$
6 \boldsymbol{F}-\mathrm{SU}(4)_{0}-\mathrm{Sp}(1)_{0}-\mathrm{Sp}(1)-2 \boldsymbol{F} .
$$

The first gauge theory description comes from string dualities, namely the circle compactification of an M5-brane probing an $\mathbb{R}^{5} / E_{6}$ singularity. We also find these two gauge theory descriptions geometrically by studying some resolutions and their rulings, as well as matching the triple intersection numbers with the putative quiver gauge theory prepotential, see appendix C. Furthermore there is a prospective description in terms of the quiver

$$
5 \boldsymbol{F}-\mathrm{Sp}(1)-\mathrm{SO}(6)-\mathrm{Sp}(1)-5 \boldsymbol{F},
$$

for which we stress that there is no geometric underpinning. Curiously, this quiver can be obtained by gluing two marginal theories of the rank one E-string, by gauging a diagonal $\mathrm{SO}(6)$

$$
\begin{aligned}
5 \boldsymbol{F}-\mathrm{Sp}(1)-3 \boldsymbol{F} \stackrel{\text { gauge diag. } \mathrm{SO}(6)}{\longleftrightarrow} & 3 \boldsymbol{F}-\mathrm{Sp}(1)-5 \boldsymbol{F} \\
& =5 \boldsymbol{F}-\mathrm{Sp}(1)-\mathrm{SO}(6)-\mathrm{Sp}(1)-5 \boldsymbol{F} .
\end{aligned}
$$

For (6.13) and (6.14) we have discussed the descendants and the dualities amongst those descendants in sections 6.1.1 and 6.1.2, and for (6.12) these were discussed in [2]. In this section we will determine, again from the structure of the CFDs in the CFD-tree, the dualities not only amongst descendants of the same marginal quiver, but also the dualities amongst the descendants of all three marginal quivers. As we have previously stated, descendants of (6.12) are captured by the tuple $\left(m_{1}, m_{2}, m_{3}\right)$, those of (6.13) by $\left(m_{1}, k, m_{2}\right)$, and finally the data of the descendants of the quiver (6.14) can be specified by $\left(m_{1}, m_{2}\right)$. For the first two class of quiver gauge theories, we further find that the prepotentials agree in some region of the respective Coulomb branches. The dualities amongst all these descendants are shown in table 5 . 


\begin{tabular}{|c|c|c|}
\hline$\left(m_{1},|k|, m_{2}\right)$ & $\left(m_{1}, m_{2}, m_{3}\right)$ & $\left(m_{1}, m_{2}\right)$ \\
\hline$(6,0,2)$ & $(2,2,2)$ & $(5,5)$ \\
\hline$(5,1 / 2,2),(6,0,1)$ & $(1,2,2)$ & $(4,5)$ \\
\hline$(5,1 / 2,1),(4,0,2)$ & $(1,1,2)$ & $(4,4)$ \\
\hline$(4,1,2),(6,0,0),(6,0, \pi)$ & $(0,2,2),(\pi, 2,2)$ & $(3,5)$ \\
\hline$(4,0,1)$ & $(1,1,1)$ & - \\
\hline$(3,3 / 2,2)$ & - & $(2,5)$ \\
\hline$(3,1 / 2,2),(4,1,1),(5,1 / 2,0),(5,1 / 2, \pi)$ & $(0,1,2),(\pi, 1,2)$ & $(3,4)$ \\
\hline$(2,0,2),(4,0,1)$ & $(0,0,2),(\pi, \pi, 2)$ & - \\
\hline$(4,1, \pi)$ & $(0, \pi, 2)$ & $(3,3)$ \\
\hline$(3,1 / 2,1),(4,0,0),(4,0, \pi)$ & $(0,1,1),(\pi, 1,1)$ & - \\
\hline$(3,3 / 2,1)$ & - & $(2,4)$ \\
\hline$(2,2,2)$ & - & $(1,5)$ \\
\hline$(2,0,1),(3,1 / 2,0)$ & $(0,0,1),(\pi, \pi, 1)$ & - \\
\hline$(3,1 / 2, \pi)$ & $(0, \pi, 1)$ & - \\
\hline$(3,3 / 2, \pi)$ & - & $(2,3)$ \\
\hline$(2,0,0)$ & $(0,0,0),(\pi, \pi, \pi)$ & - \\
\hline$(2,0, \pi)$ & $(0,0, \pi),(0, \pi, \pi)$ & - \\
\hline$(2,1,2),(3,3 / 2,1)$ & - & - \\
\hline$(1,3 / 2,2),(2,2,1)$ & - & $(1,4)$ \\
\hline$(1,5 / 2,1)$ & - & $(0,4)$ \\
\hline$(0,2,2)$ & - & $(\pi, 4)$ \\
\hline$(0,1,2),(2,2,0)$ & - & - \\
\hline$(2,2, \pi)$ & - & $(1,3)$ \\
\hline$(1,1 / 2,1),(2,1,0)$ & - & - \\
\hline$(1,5 / 2, \pi)$ & - & $(0,3)$ \\
\hline$(0,1,1),(1,3 / 2,0)$ & - & - \\
\hline
\end{tabular}

Table 5. Dualities amongst marginal quiver gauge theory descriptions of minimal $\left(E_{6}, E_{6}\right)$ conformal matter. The three columns correspond to the quiver gauge theories $(6.13),(6.12)$ and (6.14), respectively. We do not write explicitly the obvious duality between $\left(m_{1}, k, m_{2}\right)$ and $\left(m_{1},-k, m_{2}\right)$, and simply write the Chern-Simons level in terms of an absolute value.

\subsection{Descendants and dualities for minimal $\left(E_{7}, E_{7}\right)$ conformal matter}

In section 4.8 we speculated as to maximal quivers that may be marginal theories for minimal $\left(E_{7}, E_{7}\right)$ conformal matter. We found, from the embeddings of the BG-CFDs into the marginal CFD, that there are precisely two symmetric options. These were

$$
\begin{aligned}
& 6 \boldsymbol{F}-\mathrm{Sp}(5)-\mathrm{Sp}(5)-6 \boldsymbol{F} \\
& 6 \boldsymbol{F}-\mathrm{Sp}(3)-\mathrm{SO}(8)-\mathrm{Sp}(3)-6 \boldsymbol{F} .
\end{aligned}
$$

In this section we will first show that the former quiver is, in fact, inconsistent with the CFD-tree, and thus cannot be a marginal description of the $\left(E_{7}, E_{7}\right)$ theory. Secondly, 
we shall consider the latter quiver, which is consistent, and determine the superconformal flavor symmetry of its descendant quiver gauge theories.

First we rule out the quiver containing two $\mathrm{Sp}(5)$ gauge nodes. Since the bifundamental representation between the two $\mathrm{Sp}(5)$ nodes is real then there is an $\mathrm{SU}(2)$ classical flavor symmetry factor rotating that bifundamental. The descendant quiver

$$
5 \boldsymbol{F}-\mathrm{Sp}(5)-\mathrm{Sp}(5)-5 \boldsymbol{F},
$$

has classical flavor symmetry

$$
\mathrm{SO}(10)^{2} \times \mathrm{U}(1)^{2} \times \mathrm{SU}(2),
$$

and a study of the descendants of the marginal CFD informs that the superconformal flavor symmetry, if the quiver were indeed a description of minimal $\left(E_{7}, E_{7}\right)$ conformal matter, would be

$$
E_{6}^{2} \times \mathrm{U}(1) .
$$

However, there does not exist an inclusion of this classical global symmetry group into the superconformal flavor symmetry group, and thus we find a contradiction with the assumption that the quiver $6 \boldsymbol{F}-\mathrm{Sp}(5)-\mathrm{Sp}(5)-6 \boldsymbol{F}$ does have minimal $\left(E_{7}, E_{7}\right)$ conformal matter at its UV fixed point. As such, this quiver must be ruled out as a possibility.

The potential marginal quiver $6 \boldsymbol{F}-\mathrm{Sp}(3)-\mathrm{SO}(8)-\mathrm{Sp}(3)-6 \boldsymbol{F}$ does not have such an issue as there are no real bifundamental hypermultiplets which would have an $\mathrm{SU}(2)$ rotation group. From the CFD, (4.44), one finds that quivers of the form

$$
m_{1} \boldsymbol{F}-\mathrm{Sp}(3)-\mathrm{SO}(8)-\mathrm{Sp}(3)-m_{2} \boldsymbol{F},
$$

are descendants of this putative marginal quiver. For each of the following parameters $\left(m_{1}, m_{2}\right)$, they have superconformal flavor symmetries, which enhance from the classical flavor symmetry ${ }^{18}$

$$
\mathrm{SO}\left(2 m_{1}\right) \times \mathrm{SO}\left(2 m_{2}\right) \times \mathrm{U}(1)^{3},
$$

to the superconformally enhanced values for $\left(m_{1}, m_{2}\right)$ given by

$$
\begin{aligned}
(5,6): & E_{7} \times E_{7} \\
(4,6): & \mathrm{SO}(12) \times E_{7} \\
(3,6): & \mathrm{SU}(6) \times E_{7} \\
(2,6): & \mathrm{SU}(4) \times \mathrm{SU}(2) \times E_{7} \\
(1,6): & \mathrm{SU}(3) \times \mathrm{U}(1) \times E_{7} \\
(0,6): & \mathrm{SU}(3) \times \mathrm{U}(1) \times E_{7} \\
(\pi, 6): & \mathrm{SU}(2) \times \mathrm{U}(1) \times E_{7} \\
\left(m_{1}, m_{2}\right): & E^{\left(m_{1}\right)} \times E^{\left(m_{2}\right)} \times \mathrm{U}(1) \quad \text { for } m_{1}, m_{2}=0, \pi, 1,2,3,4,5 .
\end{aligned}
$$

Note that we have again used the $E^{(k)}$ shorthand for the exceptional groups, as defined in (6.9). We stress that these dualities are only determined under the assumption that the putative marginal quiver is, in fact, a quiver gauge theory which realizes minimal $\left(E_{7}, E_{7}\right)$ conformal matter at its UV fixed point. One approach to verify these dualities from first principles would be to find the associated rulings in resolutions of the $\left(E_{7}, E_{7}\right)$ geometry.

\footnotetext{
${ }^{18}$ To make sense of this expression we use the shorthand that the groups $\mathrm{SO}(0)$ and $\mathrm{SO}(\pi)$ are trivial.
} 


\subsection{Descendants and dualities for minimal $\left(E_{7}, \mathrm{SO}(7)\right)$ conformal matter}

As shown in [21], the marginal theory for $\left(E_{7}, \mathrm{SO}(7)\right)$ minimal conformal matter theory has a single gauge node description of the form $\mathrm{SU}(4)_{0}+2 \boldsymbol{A} \boldsymbol{S}+8 \boldsymbol{F}$. Here we propose a dual description as the quiver, which will be supported geometrically as well as from the CFD and by matching the prepotential, given by

$$
6 \boldsymbol{F}-\mathrm{Sp}(2)-\mathrm{Sp}(1)-2 \boldsymbol{F} .
$$

In this section we use the CFD to determine the superconformal flavor symmetries of and the dualities amongst the descendants of this quiver. Such quivers take the form

$$
m_{1} \boldsymbol{F}-\mathrm{Sp}(2)-\mathrm{Sp}(1)-m_{2} \boldsymbol{F} .
$$

This gauge theory description (as well as the $\mathrm{SU}(4)_{0}+2 \boldsymbol{A} \boldsymbol{S}+8 \boldsymbol{F}$ ) is supported by the rulings of the geometric resolutions as shown in appendix $\mathrm{C}$, and the triple intersection numbers match the putative gauge theory prepotential. As a further outcome of the prepotential analysis, we notice that there are regions of the Coulomb branch of $\mathrm{SU}(4)_{k}+2 \boldsymbol{A} \boldsymbol{S}+m \boldsymbol{F}$ and (6.24), where the prepotential coincide, provided that

$$
m_{1}=2 \pm k+\frac{m}{2}, \quad m_{2}=\mp k-2+\frac{m}{2} .
$$

\begin{tabular}{|c|c|c|}
\hline$(m,|k|)$ & $\left(m_{1}, m_{2}\right)$ & $G_{\mathrm{F}}$ \\
\hline$\left(7, \frac{1}{2}\right)$ & $(5,2),(6,1)$ & $\mathrm{SO}(7) \times E_{7}$ \\
\hline$(6,1)$ & $(4,2),(6,0)$ & $\mathrm{SO}(7) \times \mathrm{SO}(12)$ \\
\hline$(6,0)$ & $(5,1)$ & $\mathrm{SO}(5) \times E_{6} \times \mathrm{U}(1)$ \\
\hline$\left(5, \frac{3}{2}\right)$ & $(3,2)$ & $\mathrm{SO}(7) \times \mathrm{SU}(6)$ \\
\hline$(4,2)$ & $(2,2)$ & $\mathrm{SO}(7) \times \mathrm{SU}(4) \times \mathrm{SU}(2)$ \\
\hline$\left(3, \frac{5}{2}\right)$ & $(1,2)$ & $\mathrm{SO}(7) \times \mathrm{SU}(3) \times \mathrm{U}(1)$ \\
\hline- & $(0,2)$ & $\mathrm{SO}(7) \times \mathrm{SU}(3)$ \\
\hline$(2,3)$ & $(\pi, 2)$ & $\mathrm{SO}(7) \times \mathrm{SU}(2) \times \mathrm{U}(1)$ \\
\hline$\left(5, \frac{1}{2}\right)$ & $(4,1),(5,0)$ & $\mathrm{SO}(5) \times \mathrm{SO}(10) \times \mathrm{U}(1)$ \\
\hline$\left(1+m, \frac{5-m}{2}\right)$ & $(m, 1)$ & $\mathrm{SO}(5) \times E_{m+1} \times \mathrm{U}(1) \quad m=1,2,3$ \\
\hline$\left(1, \frac{5}{2}\right)$ & $(\pi, 1)$ & $\mathrm{SO}(5) \times \mathrm{U}(1) \times \mathrm{U}(1)$ \\
\hline- & $(0,1)$ & $\mathrm{SO}(5) \times \mathrm{SU}(2) \times \mathrm{U}(1)$ \\
\hline$\left(m, \frac{4-m}{2}\right)$ & $(m, 0)$ & $\mathrm{SO}(5) \times \mathrm{SO}(2 m) \times \mathrm{U}(1) \quad m=1, \cdots, 4$ \\
\hline$(0,2)$ & $(\pi, 0)$ & $\mathrm{SO}(5) \times \mathrm{U}(1)$ \\
\hline- & $(0,0)$ & $\mathrm{SO}(5) \times \mathrm{U}(1)$ \\
\hline- & $(6, \pi)$ & $\mathrm{SU}(2)^{2} \times E_{7}$ \\
\hline- & $(m, \pi)$ & $\mathrm{SU}(2) \times E^{(m)} \times \mathrm{U}(1) \quad m=0, \pi, 1, \cdots, 5$ \\
\hline
\end{tabular}

The superconformal flavor symmetries and the dualites for such gauge theories are ${ }^{19}$

\footnotetext{
${ }^{19}$ Recall once more our convention that $\mathrm{SO}(x)$ for $x$ not a positive integer is the trivial group.
} 


\subsection{Dualities for the marginal theory of $\mathrm{SU}(2 n+1)_{0}+2 A S+8 F$}

The gauge theory, $\mathrm{SU}(2 n+1)_{0}+2 \boldsymbol{A} \boldsymbol{S}+8 \boldsymbol{F}$, and its $6 \mathrm{~d}$ origin have been discussed in section 5.3, where we have also shown all the possible embeddings of classical flavor symmetries for putative dual theories, see figure 23. To test examples in this infinite class of dualities via checks of the prepotential, we specialize to $n=2$ and compare the proposed dual theories. In fact, the prepotential matches in non-trivial regions of the respective Coulomb branches for the following quivers:

$$
\begin{aligned}
& 4 \boldsymbol{F}-\operatorname{Sp}(2)-\operatorname{Sp}(1)_{\theta}-\operatorname{Sp}(1)-2 \boldsymbol{F} \\
& 4 \boldsymbol{F}-\mathrm{SU}(3)_{2}-\operatorname{Sp}(1)_{\theta}-\mathrm{Sp}(1)-2 \boldsymbol{F} \\
& 8 \boldsymbol{F}-\operatorname{Sp}(3)-\operatorname{Sp}(1)_{\theta} \\
& 8 \boldsymbol{F}-\mathrm{SU}(4)_{1}-\operatorname{Sp}(1)_{\theta} \\
& 4 \boldsymbol{F}-\operatorname{Sp}(2)-\operatorname{Sp}(2)-4 \boldsymbol{F} \\
& \qquad[2 \boldsymbol{F}] \\
& \quad \mid \\
& 2 \boldsymbol{F}-\operatorname{Sp}(1)-\operatorname{Sp}(1)_{\theta}-\operatorname{Sp}(1)-\operatorname{Sp}(1)_{\theta}
\end{aligned}
$$

The dualities should hold for specific values of the $\theta$-angles, even if the prepotential matching is not sufficient to resolve this ambiguity. This gives strong evidence that our method of embedding the BG-CFDs of a $5 \mathrm{~d}$ gauge theory with classical flavor symmetry into the CFD is particularly useful and efficient in predicting candidate 5d UV-dual theories.

\subsection{Dualities for the marginal theory of $\mathrm{SU}(2 n)_{0}+2 A S+8 F$}

Similarly to the previous section, we now focus on the following theory $\mathrm{SU}(2 n)_{0}+2 \boldsymbol{A} \boldsymbol{S}+8 \boldsymbol{F}$, which has been already discussed in section 5.2. In particular, we test here the predictions for the existence of alternative effective gauge theory descriptions, which come from embedding the classical flavor symmetry into the CFD, (5.10). These embeddings have been proposed in figure 20. Specializing to the case $n=3$, we can explicitly compute the prepotential, and, for instance, we find that the prepotential for $\mathrm{SU}(6)_{0}+2 \boldsymbol{A} \boldsymbol{S}+8 \boldsymbol{F}$ is consistent with the following quivers:

$$
\begin{aligned}
& 6 \boldsymbol{F}-\mathrm{SU}(4)_{1}-\mathrm{Sp}(1)_{\theta}-\mathrm{Sp}(1)_{\theta} \\
& 6 \boldsymbol{F}-\mathrm{SU}(4)_{1}-\mathrm{Sp}(2)-2 \boldsymbol{F} \\
& 2 \boldsymbol{F}-\mathrm{Sp}(2)-\mathrm{Sp}(3)-6 \boldsymbol{F}
\end{aligned}
$$

Again, the dualities will hold for specific values of the $\theta$-angles, which have to be determined by alternative methods. We observe here, that the proposed duals match with the embeddings of the classical flavor symmetry as dictacted by the embedding of BG-CFDs into the CFDs as shown in figure 20 . 


\section{Fibers from Coulomb branch phases}

In the previous sections, we have heavily employed the description in terms of the BGCFDs, which are graphs associated to gauge theories, in order to study their SCFT limits in terms of CFDs. The connection between these two related concepts is based on the M-theory description underlying both. In particular, we will discuss in this section how the box graphs encode the geometry for the BG-CFDs, and how, with minimal additional input, it also determines the superconformal flavor symmetry enhancement.

To begin with, we briefly recall the necessary background material on non-minimal singularities in elliptic fibrations, their (possibly non-flat) resolutions, and their role in engineering $5 \mathrm{~d}$ SCFTs, and refer to $[2,12]$ for more details.

\subsection{5d SCFTs and M-theory on elliptic Calabi-Yau threefolds}

We can associate a singular, non-compact elliptic Calabi-Yau threefold $\pi: Y \rightarrow B$ to each marginal theory, whose 6d UV-completion is described by F-theory compactified on $Y$. Different mass deformations of the marginal theory, which results in different $5 \mathrm{~d}$ SCFTs pushed onto their Coulomb branches, correspond to different crepant resolutions $\widehat{Y} \rightarrow Y$. The resolution introduces compact and non-compact surfaces/divisors, $S_{j}$ and $D_{i}^{(\nu)}$, respectively. In fact, the compact reducible surface $\mathcal{S}=\bigcup_{j} S_{j}, j=1, \cdots, r$, fully determines the (local) geometry and completely characterizes the 5d SCFT. In particular, the gauge group $G_{\text {gauge }}$ is determined by how each surface $S_{j}$ is ruled, i.e., fibered by $\mathbb{P}_{j}^{1} \equiv f_{j}[40] .{ }^{20}$ There are generally multiple compatible rulings, denoted by $f_{j}^{(n)} \hookrightarrow S_{j}$, which correspond to different effective gauge theories with the same SCFT limit, i.e., that are UV-dual to each other.

The smooth geometry $\widehat{Y}$ corresponds to a generic point on the Coulomb branch, where the effective description is just a $\mathrm{U}(1)^{r}$ theory with no charged light states. By partially blowing down all rulings $f_{j}$ of the compact surfaces, thus shrinking $S_{j}$ to a curve, the gauge symmetry enhances to the full non-abelian group $G_{\text {gauge }}$. M2-branes wrapping holomorphic curves that collapse in this limit give rise to massless charged hypermultiplets and the Wbosons of $G_{\text {gauge }}$. By further collapsing $S_{j}$ to a point, the theory becomes a strongly coupled $5 \mathrm{~d}$ SCFT, as the volume is inversely proportional to the gauge coupling.

At each stage of the two-step collapse, it can happen that non-compact divisors $D_{i}^{(\nu)}$ are also forced to shrink, leading to canonical surface singularities along a non-compact curve in the threefold, whose singularity type encodes the flavor symmetry [42]. In particular, the singularity type can at most become worse when $S_{j}$ is collapsed first to a curve and then to a point. This reflects the field theory intuition on the enhancement $G_{\mathrm{F}, \mathrm{cl}} \subseteq G_{\mathrm{F}}$ from classical to superconformal flavor symmetry.

The key proposal of [12] to read off the flavor symmetry geometrically, which was systematized and condensed into CFDs in [1,2], is to track how the non-compact divisors $D_{i}^{(\nu)}$ intersect $\mathcal{S}$. More precisely, these divisors are $\mathbb{P}^{1}$-fibered over a non-compact curve $W_{\nu} \subset B$. Over a generic point on $W_{\nu}$, the fibers $F_{i}^{(\nu)}$ intersect in an affine Dynkin diagram $\widehat{G}_{\mathrm{F}, \nu}^{(6 \mathrm{~d})}$. In

\footnotetext{
${ }^{20}$ See also section 2.2 of [2] for a summary in the same notation as here.
} 
F-theory, these determine the $6 \mathrm{~d}$ superconformal flavor symmetry $G_{\mathrm{F}}^{(6 \mathrm{~d})}=\prod_{\nu} G_{\mathrm{F}, \nu}^{(6 \mathrm{~d})}$. By construction, a non-compact divisor $D_{i}^{(\nu)}$ intersects the compact surfaces $\bigcup_{j} S_{j}=\mathcal{S}$ over isolated points $p \in W_{\nu}$. Depending on the resolution, the $\mathbb{P}^{1}$ fiber $F_{i}^{(\nu)}$ may or may not be contained in $\mathcal{S}$. Those $F_{i}^{(\nu)}$ that are contained form the non-affine Dynkin diagram of $G_{\mathrm{F}, \nu}$. Since these fibers shrink everywhere over $W_{\nu}$ when $\mathcal{S}$ is collapsed to a point, they determine the non-abelian part of the $5 \mathrm{~d}$ superconformal flavor symmetry, $G_{\mathrm{F}, \mathrm{na}}=\prod_{\nu} G_{\mathrm{F}, \nu}$. We will refer to these curves, as in [2], as flavor curves.

Abelian factors of the full $5 \mathrm{~d}$ superconformal flavor symmetry $G_{\mathrm{F}}$ come from noncompact divisors $D_{i}^{(\nu)}$ whose fibers are not fully contained in $\mathcal{S}$, but nevertheless intersects $\mathcal{S}$ in curves. In general, there can be linear redundancies amongst different such divisors, which can be inferred from the intersection numbers of all curves inside $\mathcal{S}$ and all divisors, see $[2,12]$. In practice, we know on general grounds the full rank of $G_{\mathrm{F}}$ from the classical flavor symmetry $G_{\mathrm{F}, \mathrm{cl}}$ and the number $N$ of topological $\mathrm{U}(1) \mathrm{s}$,

$$
\operatorname{rank}\left(G_{\mathrm{F}}\right)=\operatorname{rank}\left(G_{\mathrm{F}, \mathrm{cl}}\right)+N
$$

so that the number of $\mathrm{U}(1)$ factors is determined $\operatorname{as} \operatorname{rank}\left(G_{\mathrm{F}}\right)-\operatorname{rank}\left(G_{\mathrm{F}, \mathrm{cl}}\right)$.

\subsection{Fibers from box graphs}

Consider now a marginal theory with symmetry $G_{\text {gauge }} \times G_{\mathrm{BG}}$ (where $G_{\mathrm{BG}}$ is the classical flavor symmetry of the marginal theory). We now determine from the box graphs, that are associated to each descendant theory, the intersection structure amongst the divisors $S_{j}$ and $D_{i}^{(\nu)}$, which in particular specifies which codimension one fibers $F_{i}^{(\nu)}$ are contained in $\mathcal{S}$, i.e., which are flavor curves. Both $S_{j}$ and $D_{i}^{(\nu)}$ are ruled surfaces, compact and non-compact, respectively. The fibers are denoted by $f_{j}$ and $F_{i}^{(\nu)}$, respectively and we introduce the notation

$$
\mathcal{D}_{\ell} \in\left\{S_{j}, D_{i}^{(\nu)}\right\}, \quad \mathcal{F}_{\ell} \in\left\{f_{j}, F_{j}^{(\nu)}\right\}
$$

for all divisors and fibral curves. Next, recall the relationship between the representation theory of a Lie group $G$ and intersection theory in M-theory on a smooth Calabi-Yau threefold $\widehat{Y}$ : to each ruled surface $\mathcal{F}_{\ell} \hookrightarrow \mathcal{D}_{\ell}$, we associate a simple root $\alpha_{\ell}$ to the curve $\mathcal{F}_{\ell}$ (with normal bundle $\mathcal{O} \oplus \mathcal{O}(-2)$ inside $\widehat{Y}$ ), and its coroot $\alpha_{\ell}^{\vee}$ to the divisor $\mathcal{D}_{\ell}$, such that

$$
\mathcal{C}_{\ell \kappa}^{G} \equiv\left\langle\alpha_{\kappa}^{\vee}, \alpha_{\ell}\right\rangle=-\mathcal{D}_{\kappa} \cdot \mathcal{F}_{\ell}
$$

where $\mathcal{C}_{\ell \kappa}^{G}$ is the Cartan matrix of $G$. In our setup, the (co-)roots of $G_{\text {gauge }}$ arise from $\mathcal{D}_{\ell}=S_{j}$, whereas those of $G_{\mathrm{BG}}$ come from $\mathcal{D}_{\ell}=D_{i}$.

The pairing between weights and coweights is identified with the intersection pairing between divisors and curves. Consider a box graph associated to a representation of $G_{\text {gauge }} \times G_{\mathrm{BG}}$. To an extremal weight $L$ in the box graph (see definition 2.3) we associate a curve $C$ with normal bundle $\mathcal{O}(-1) \oplus \mathcal{O}(-1)$, and refer to such a curve as an extremal curve. Denote by $L^{ \pm}= \pm L$, where in a given box graph only one of these is in the cone 


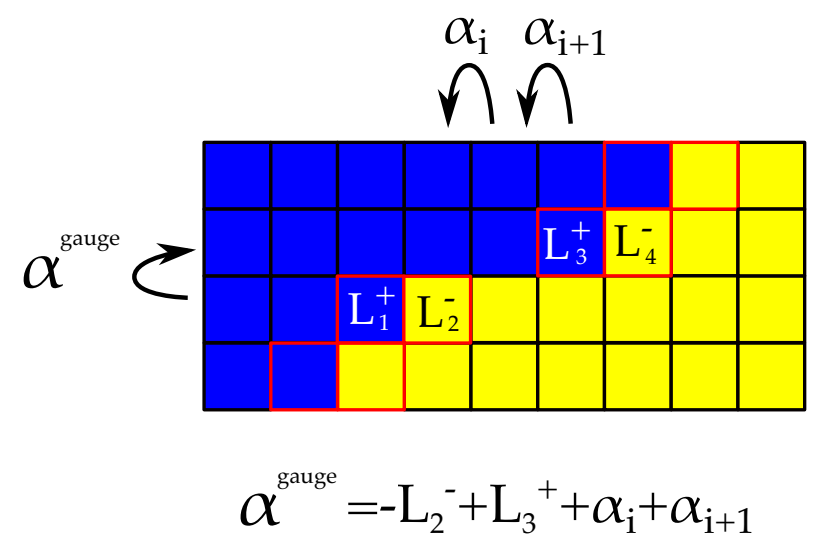

Figure 25. Example box graph for $\mathrm{SU}(n)_{\text {gauge }} \times \mathrm{U}(m)_{\mathrm{BG}}$ : the root $\alpha^{\text {gauge }}$ of the gauge algebra $\mathrm{SU}(n)$ splits. The extremal weights are boxed in red. The splitting of $\alpha^{\text {gauge }}$ into the extremal weights $L_{2}^{-}$and $L_{3}^{+}$, as well as the roots of $G_{\mathrm{BG}}, \alpha_{i}$, is determined from the box graph. The arrows indicate, as usual for box graphs, addition of roots. In the geometry, we associate to $\alpha^{\text {gauge }}$ the curves $f_{i}$ and to $\alpha_{j}$ the curves $F_{j}$.

defining the Coulomb branch, and associated to that the curve $C^{ \pm}= \pm C$ (which is an effective curve if the corresponding weight is in the cone), related by

$$
\left(L^{ \pm}\right)_{\ell} \equiv \pm\left\langle\alpha_{\ell}^{\vee}, L\right\rangle=\mp \mathcal{D}_{\ell} \cdot C^{ \pm}
$$

All other weights are then realized as the linear combination of extremal curves and $\mathcal{F}_{\ell}$.

A curve $\mathcal{F}$ associated to a root, which can be either a codimension one fiber $F_{i}^{(\nu)}$ or a ruling $f_{j}$ of the surface $S_{j}$, is reducible in codimension two, if the associated box graph indicates a splitting

$$
\mathcal{F}=\sum_{a} n_{a} C_{a}^{\epsilon_{a}}+\sum_{\nu} \sum_{i} \omega_{i}^{(\nu)} F_{i}^{(\nu)}+\sum_{j} \eta_{j} f_{j}, \quad \omega_{i}, \eta_{j} \geq 0 .
$$

The first sum is over all extremal curves $C_{a}^{\epsilon_{a}}$, where $\epsilon_{a}= \pm$. An example is shown in figure 25.

If $\mathcal{F}=f_{j}$ is the ruling of a compact divisor $S_{j}$, then we can immediately deduce that all curves appearing on the right-hand side of (7.5) must be contained in $S_{j}$, and thus in $\mathcal{S}$. In particular, the simple roots of the classical flavor symmetry $G_{\mathrm{F}, \mathrm{cl}}$ are precisely those $F_{i}^{(\nu)}$ into which an $f_{j}$ splits via (7.5). In the example of figure 25 these are the roots associated to $F_{i}$ and $F_{i+1}$.

As for the superconformal flavor symmetry, recall that the Dynkin diagram of $G_{\mathrm{BG}}-$ which is also the classical flavor symmetry of the marginal theory - fills only a subpart of the affine Dynkin diagram $\widehat{G}_{\mathrm{F}}^{(6 \mathrm{~d})} \equiv \prod_{\nu} \widehat{G}_{\mathrm{F}, \nu}^{(6 \mathrm{~d})}$. We denote divisors that are not captured in the box graph, but are also ruled non-compact surfaces, by

$$
F_{\Phi_{l}} \hookrightarrow D_{\Phi_{l}}
$$

In order to distinguish them from the roots/Cartans of $G_{\mathrm{BG}}$, these will be denoted by $F_{i} \hookrightarrow D_{i}$ in the following. To encode in the box graph approach the full superconformal 
flavor symmetry requires determining how these additional nodes are attached to the part of the fiber that we reconstruct from the box graph.

Physically, these "missing" divisors either correspond to abelian factors of the classical flavor symmetry (and hence have no roots that appear in the box graph), or even the nonperturbative instanton U(1)s associated with each simple factor of $G_{\text {gauge }}$. Nevertheless, they can be fully contained in $\mathcal{S}$ (as (multi-)sections of the rulings), and thus lead to an enhanced superconformal flavor group.

The precise nature of the curves $F_{\Phi_{l}}$ (e.g., the information on how they split, or in which surface $S_{j}$ they are contained) is dictated by consistency conditions from intersection theory. For that, we need one piece of information which is not contained in the box graphs, namely the intersection numbers $D_{\Phi_{l}} \cdot C$, where $C$ is an extremal curve. These numbers depend on the resolution phase, as the extremal curves $C$ are different in each phase. However, what remains invariant throughout all phases is $D_{\Phi_{l}} \cdot C(L)$, where $C(L)$ is the curve (possibly reducible) associated to a particular weight $L$ in the box graph. This is because the linear combinations of divisors corresponding to abelian flavor symmetries must give rise to well-defined charges for all weights $L \in \boldsymbol{R}$ of the box graph. These linear combinations do not change across different resolutions, as they are - similar to the Shiodamap for U(1)s in F-theory - divisorial, i.e., codimension one data. Correspondingly, the charges of the individual weights $\boldsymbol{R}$ under each $D_{\Phi_{l}}$ must remain invariant. In practice, we therefore compute $D_{\Phi_{l}} \cdot C(L)$ for all $l$ and weights $L$. This has to be done in one specific resolution, e.g., for the marginal theory having a $G_{\text {gauge }}$ gauge description, or indeed for any other resolution.

This data can also be phrased representation-theoretically. We can extend the (co)weight lattice by additional (co-)roots $\Phi_{l}^{\vee}$ and $\Phi_{l}$, such that every weight $L_{m}$ carries additional charges given by the pairing

$$
\left\langle\Phi_{l}^{\vee}, L_{m}\right\rangle .
$$

The holomorphic curve $C\left(L_{m}^{\epsilon_{m}}\right)$ that corresponds to a decoration $\epsilon_{m}= \pm 1$ of $L_{m}$ then has, as in (7.4), the intersection

$$
\mathcal{E}_{l, m}=D_{\Phi_{l}} \cdot C_{m}^{\epsilon_{m}}=-\epsilon_{m}\left\langle\Phi_{l}^{\vee}, L_{m}\right\rangle .
$$

In concrete examples, we will provide the numbers $\mathcal{E}_{l, m}$, as determined by any one specific resolution, for the associated undecorated box graph.

With these numbers, the fiber geometry can be deduced from well-known intersection properties of elliptically fibered threefolds. Firstly, we know that the intersection numbers

$$
\widehat{\mathcal{C}}_{\ell \kappa}^{(6 \mathrm{~d})}=-\mathcal{F}_{\ell} \cdot \mathcal{D}_{\kappa}, \quad \text { with } \mathcal{F}_{\ell} \in\left\{F_{i}, F_{\Phi_{l}}\right\} \text { and } \mathcal{D}_{\kappa} \in\left\{D_{i}, D_{\Phi_{l}}\right\},
$$

form the affine Cartan matrix of $\widehat{G}_{\mathrm{F}}^{(6 \mathrm{~d})}$. Secondly, we have

$$
S_{j} \cdot \mathcal{F}_{\ell}=0 \quad \text { for } \mathcal{F}_{\ell} \in\left\{F_{i}, F_{\Phi_{l}}\right\}
$$

Finally, we know from the factorization $G_{\text {gauge }} \times G_{\mathrm{BG}}$ that

$$
D_{i} \cdot f_{j}=S_{j} \cdot F_{i}=0 \quad \text { for all } i, j .
$$


With the above information, we can apply in each phase the following rules from intersection theory to determine the fiber structure:

1. If an irreducible curve has negative intersection with a divisor, $C \cdot \mathcal{D}<0$, then $C$ must be contained in $\mathcal{D}$.

2. If a ruling of a surface (compact or not) splits as in (7.5), then all curves into which it splits must form a connected curve.

3. If a codimension one fiber $\mathcal{F}_{\ell}$ does not split, then $\mathcal{D}_{\ell} \cdot C \geq 0$ for any curve $C \neq \mathcal{F}_{\ell}$, such that $C$ is not contained inside of $\mathcal{D}_{\ell}$. If $\mathcal{D}_{\ell} \cdot C=n>0$, then $C$ and $\mathcal{F}_{\ell}$ intersect in $n$ points.

4. If a non-splitting codimension one fiber $\mathcal{F}$ intersects a curve $C$ contained in a compact surface $S_{j}$, then $\mathcal{F} \subset S_{j}$, and is a flavor curve.

While the first three points follow from basic algebraic geometry, the last point is due to the intersection number (7.10): if $\mathcal{F}$ intersects a curve $C \subset S$, the only way to preserve this intersection number is if $\mathcal{F}$ is also contained. By these rules, it is straightforward to reconstruct the configurations of the compact surfaces $S_{j}$ and the relative positions of the codimension one fibers $\mathcal{F}=F_{i}, F_{\Phi_{l}}$.

As a last comment, note that the intersection pattern of extremal curves and the roots $F_{i}$ of $G_{\mathrm{BG}}$ contained in $\mathcal{S}$ precisely form the BG-CFD. These curves shrink when we blow down the surfaces $S_{j}$ to curves in order to have non-abelian gauge enhancement. Conversely, if a geometry is supposed to contain a specific gauge description, then it must contain the BG-CFD as a subset of curves.

In summary our strategy will be as follows:

For the marginal $5 \mathrm{~d}$ theories, we determine the geometric resolution. The flavor symmetry $G_{\mathrm{F}}^{(6 \mathrm{~d})}$ of the parent $6 \mathrm{~d}$ theory will be manifest in this description. Different rulings of the surfaces yield different weakly coupled gauge theory descriptions with $G_{\text {gauge }} \times G_{\mathrm{BG}}$. The geometric resolution provides the following data:

- The embedding of the BG-CFD of $G_{\mathrm{BG}}$ into $G_{\mathrm{F}}^{(6 \mathrm{~d})}$ (i.e., the information about how the curves $F_{\Phi_{l}}$ are attached to the curves associated to the roots of $G_{\mathrm{BG}}$ ),

- Pairings $\left\langle\Phi_{l}^{\vee}, L\right\rangle$ for all weights $L$.

To determine the descendant 5 d SCFTs with an effective $G_{\text {gauge }}$ description, we first construct all (flavor equivalence classes of) Coulomb branch phases. For each descendant, self-consistency of the box graphs and intersections of $\Phi_{\ell}$ fixes the fiber, and thus the full superconformal flavor symmetry enhancement in the SCFT limit.

\subsection{Fiber reconstruction for rank one SCFTs}

In the following, we will first discuss in detail how these methods apply to 5d SCFTs of rank one. The marginal geometry, descending from the $6 \mathrm{~d}$ rank one E-string, has an $\mathrm{SU}(2)_{\text {gauge }} \times \mathrm{SO}(16)_{\mathrm{F}}$ gauge theory description. The corresponding $\mathrm{SU}(2)_{\text {gauge }} \times \mathrm{SO}(16)_{\mathrm{BG}}$ box graphs have been presented previously in section 2.4 . 
To translate them into geometry, we first associate to the simple roots the rational curves

$$
\begin{aligned}
& \mathrm{SO}(16)_{\mathrm{BG}}: \quad F_{i} \leftrightarrow \quad-\alpha_{i}, \quad i=1, \cdots, 8, \\
& \mathrm{SU}(2)_{\text {gauge }}: \quad f \quad \leftrightarrow \quad-\alpha_{\mathrm{SU}(2)},
\end{aligned}
$$

where $f \hookrightarrow S$ is the ruling of the compact surface introduced in the resolution of the non-minimal singularity of the elliptic threefold at the $\left(E_{8}, I_{1}\right)$ collision (see [2] for more details). The $F_{i}$ rule non-compact divisors $D_{i}$ resolving the codimension one $E_{8}$ singularity and intersect in the non-affine Dynkin diagram of $\mathrm{SO}(16)$. They are embedded into the affine $E_{8}$ fiber as follows:

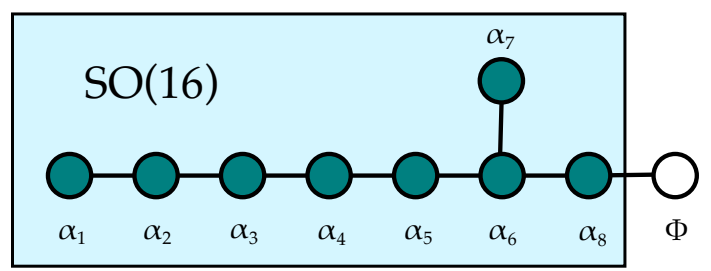

The intersection numbers $\left(D_{i} \cdot F_{j}\right)_{i j}$ give the negative Cartan matrix of $\mathrm{SO}(16)$. The additional node $F_{\Phi} \hookrightarrow D_{\Phi}$ corresponds to the "extra" root of $E_{8}$ missed by the $\mathrm{SO}(16)_{\mathrm{BG}}$ embedding (see (7.13)) and has intersection numbers

$$
D_{\Phi} \cdot F_{\Phi}=-2, \quad D_{i} \cdot F_{\Phi}=D_{\Phi} \cdot F_{i}=\delta_{i 8} .
$$

These intersection numbers are independent of the resolution phase, as they pertain to the codimension one fibers. Likewise, we have for every phase

$$
D_{i} \cdot f=S \cdot F_{i}=S \cdot F_{\Phi}=0, \quad i=1, \cdots, 8 .
$$

The different phases are given by the decorated box graphs in figure 2. In the following, we construct the fibers for these phases. The result, including the flavor group $G_{\mathrm{F}}$ of the SCFT (if existent), is listed in table 6, together with the decorated box graphs for completeness.

Phase I. The box graph for phase I is

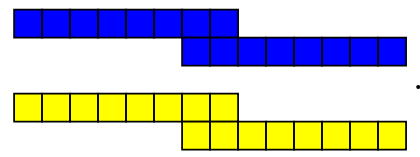

The only curve that splits in codimension two is the root of the $\mathrm{SU}(2)$, i.e., $f$, which maps from the lower representation graph of the $\mathbf{1 6}$ to the upper. There are two extremal weights, which are identified due to the pseudo-reality of the representation

$$
L_{1,16}^{+}=L_{2,1}^{-} .
$$

For definiteness, we will work with $L_{2,1}^{-}$, and associate a minus-sign with the corresponding extremal curve $C_{2,1}^{-} \equiv C^{-}$. 


\begin{tabular}{|c|c|c|c|c|}
\hline Phase & Topology of $S$ & Codim. 2 Fiber & Box Graph & $G_{\mathrm{F}}$ \\
\hline I & $\operatorname{gdP}_{9}$ & (1)-0-0-0-0-0 & 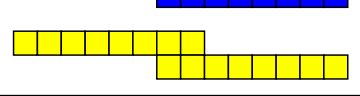 & \\
\hline II & $\operatorname{gdP}_{8}$ & (1)-0) $-0-0-C$ & 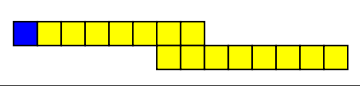 & $E_{8}$ \\
\hline III & $\operatorname{gdP}_{7}$ & 0 (1)-010-0-0 & 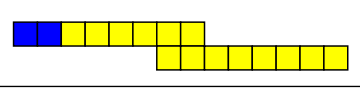 & $E_{7}$ \\
\hline IV & $\operatorname{gdP}_{6}$ & $0-0 \div 0-0$ & प1पामाम & $E_{6}$ \\
\hline $\mathrm{V}$ & $\operatorname{gdP}_{5}$ & $0-0-0 \div 0-0$ & पामा & $\mathrm{SO}(10)$ \\
\hline VI & $\operatorname{gdP}_{4}$ & $0-0-0-0$ & 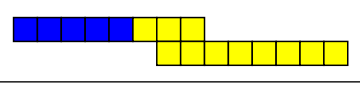 & $\mathrm{SU}(5)$ \\
\hline VII & $\operatorname{gdP}_{3}$ & 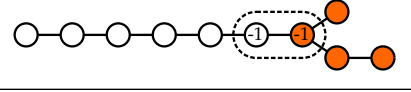 & & $\mathrm{SU}(2) \times \mathrm{SU}(3)$ \\
\hline VIII & $\operatorname{gdP}_{2}$ & $0-0-0-0-0-0)(1) \div 0$ & $\square \sqcap \square$ & $\mathrm{SU}(2) \times \mathrm{U}(1)$ \\
\hline IX & $\mathrm{dP}_{1}$ & $0-0-0-0-0-0 \div 0 \div=0$ & 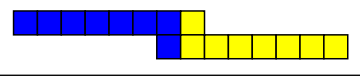 & $\mathrm{U}(1)$ \\
\hline $\mathrm{X}$ & $\operatorname{gdP}_{1} \cong \mathbb{F}_{2}$ & $\mathrm{O}-\mathrm{O}-\mathrm{O}-\mathrm{O}-\mathrm{O}-\mathrm{O}-\mathrm{O}-\mathrm{O}-\mathrm{O}-\mathrm{O}$ & ${ }_{\square}$ & $\mathrm{SU}(2)$ \\
\hline
\end{tabular}

Table 6. Box graphs and codimension two fibers corresponding to the rank one 5d SCFTs. The surface $S$ with given topology, which in an M-theory realization would supply the $\mathrm{SU}(2)$ gauge theory description, contains the codimension two fiber that is shown. Obtained in [2] from non-flat resolutions, all curves have self-intersection -2 inside $S$, except when otherwise noted. The orange colored rational curves are those contained in the surface component $S$. The flavor curves are the fully contained $(-2)$-curves (colored and unlabeled). We furthermore list the box graph/Coulomb branch phase for the gauge theory description of $\mathrm{SU}(2)_{\text {gauge }} \times \mathrm{SO}(16)_{\mathrm{BG}}$ with matter in the $(\mathbf{2}, \mathbf{1 6})$. The last column contains the enhanced flavor symmetry $G_{\mathrm{F}}$ of the $5 \mathrm{~d}$ SCFT obtained from shrinking $S$. 
The box graph dictates the following splitting for $f$

$$
f \rightarrow 2 C^{-}+2 F_{1}+2 F_{2}+2 F_{3}+2 F_{4}+2 F_{5}+2 F_{6}+F_{7}+F_{8},
$$

where the only non-zero intersections of $C^{-}$are

$$
\left\langle\alpha_{1}^{\vee}, L_{2,1}\right\rangle=D_{1} \cdot C^{-}=1, \quad\left\langle\alpha_{\mathrm{SU}(2)}^{\vee}, L_{2,1}\right\rangle=S \cdot C^{-}=-1 .
$$

Thus, the extremal curve $C^{-}$is attached to $F_{1}$, and is contained in $S$. By rule 4 in the previous subsection, all $F_{i}$ must be contained in $S$ as well.

It remains to analyze the fate of $F_{\Phi}$ - the extra curve, that is not contained in the data of the gauge theory description. Without any further input, we have to consider the explicit resolution presented in Part I [2], where the geometry was determined to be $\mathrm{gdP}_{9}$. The geometry implies that $F_{\Phi}$ is irreducible and is fully contained in $S$. The corresponding fiber is depicted at the top of table 6 .

With this geometric input, we can compute the intersections $\left\langle\Phi^{\vee}, L_{i, j}\right\rangle$ for all weights $L_{i, j}$ of $\mathrm{SU}(2)_{\text {gauge }} \times \mathrm{SO}(16)_{\mathrm{BG}}$, which we can use for the subsequent phases. First, there are sixteen effective curve classes corresponding to the weights $L_{2, j}^{-}, j=1, \ldots, 16$ (see figure 1) corresponding to the eight $\mathrm{SU}(2)_{\text {gauge }}$ flavors which transform as a half-hypermultiplet in the bifundamental of $\mathrm{SU}(2)_{\text {gauge }} \times \mathrm{SO}(16)_{\mathrm{BG}}$. In the decorated box graph (7.16), they are:

$$
\begin{aligned}
L_{2,1}^{-} & \leftrightarrow C^{-} \\
L_{2,2}^{-} & \leftrightarrow C^{-}+F_{1} \\
\vdots & \\
L_{2,8}^{-} & \leftrightarrow C^{-}+\sum_{i=1}^{7} F_{i} \\
L_{2,9}^{-} & \leftrightarrow C^{-}+\sum_{i=1}^{6} F_{i}+F_{8} \\
L_{2,10}^{-} & \leftrightarrow C^{-}+\sum_{i=1}^{6} F_{i}+2 F_{7}+F_{8} \\
L_{2,11}^{-} & \leftrightarrow C^{-}+\sum_{i=1}^{5} F_{i}+\sum_{i=6}^{7} 2 F_{i}+F_{8} \\
\vdots & \\
L_{2,16}^{-} & \leftrightarrow C^{-}+\sum_{i=1}^{7} 2 F_{i}+F_{8} .
\end{aligned}
$$

From the codimension one affine $E_{8}$ fiber, we know that $D_{\Phi} \cdot F_{8}=1$ and $D_{\Phi} \cdot F_{i}=0$ for $i \neq 8$, and furthermore we determined that $D_{\Phi} \cdot C^{-}=0$. Thus, the curves in (7.20) associated to the weights $L_{2, j}$ of the box graphs have intersections with the additional divisor $D_{\Phi}$ as follows

$$
D_{\Phi} \cdot C\left(L_{2, j}^{-}\right)=\left\langle\Phi^{\vee}, L_{2, j}\right\rangle= \begin{cases}0, & \text { if } j<9 \\ 1, & \text { if } j \geq 9\end{cases}
$$


Finally, the box graphs also determine the charges of the conjugate states via $L_{1, j+8}=-L_{2,9-j}$.

Phases II-VII. Denote the phase given by the Roman numeral corresponding to $n+1$ by the Arabic numeral $n$, i.e., phase II corresponds to $n=1$, phase III to $n=2$, etc. Then the splitting dictated by the box graph is

$$
\begin{aligned}
F_{n} & \rightarrow C_{1,16-n}^{+}+C_{1,17-n}^{-}, \\
f & \rightarrow 2 C_{1,16-n}^{+}+F_{7}+F_{8}+\sum_{j=n+1}^{6} 2 F_{j} .
\end{aligned}
$$

Recall $F_{i}$ are the curves associated to the roots of the $\mathrm{SO}(16)_{\mathrm{BG}}$, which act along the $\mathbf{1 6}$ representation, and $f$ the one of the $\mathrm{SU}(2)_{\text {gauge }}$. The intersections of the curves with the Cartan divisors $D_{i}$ and $S$ can be read off from the associated weight in the box graph. From figure 1, the non-zero numbers are

$$
\begin{aligned}
& \left(D_{n-1}, D_{n}, D_{n+1}, S\right) \cdot C_{1,16-n}^{+}=(0,-1,1,-1), \\
& \left(D_{n-1}, D_{n}, D_{n+1}, S\right) \cdot C_{1,17-n}^{-}=(1,-1,0,1) .
\end{aligned}
$$

For $n=1$ there is of course no $D_{0}$, and these terms are thus ignored. Furthermore, (7.21) implies $D_{\Phi} \cdot C_{1,16-n}^{+}=D_{\Phi} \cdot C_{1,17-n}^{-}=0$ for $1 \leq n \leq 6$. This means $F_{\Phi}$ does not split into any of these components. Similarly as in the case for Phase I, the curve $F_{8}$ is contained inside of $S$, and thus $F_{\Phi}$, since it does not split, must also be contained. The fibers and box graphs are shown in table 6 .

The weakly coupled $5 \mathrm{~d}$ gauge theory of these phases is $\mathrm{SU}(2)_{\text {gauge }}+(8-n) \mathbf{F}$. Therefore the weakly coupled flavor group is $G_{\mathrm{F}, \mathrm{cl}}=\mathrm{SO}(16-2 n)$, which including the $\mathrm{U}(1)_{T}$ gives rank $M=9-n$ for $G_{\mathrm{F}}$. In these phases, all ranks are accounted for by the shrinking simple roots including $\Phi$, so there is no abelian factor and one has $G_{\mathrm{F}}=E_{9-n}$.

Phase VIII. In this phase the splitting is

$$
\begin{aligned}
F_{7} & \rightarrow C_{1,9}^{+}+C_{1,10}^{-}, \\
F_{8} & \rightarrow C_{1,8}^{+}+C_{1,10}^{-}, \\
f & \rightarrow C_{1,8}^{+}+C_{1,9}^{+},
\end{aligned}
$$

with non-trivial intersection numbers

$$
\begin{aligned}
\left(D_{6}, D_{7}, D_{8}, S\right) \cdot C_{1,8}^{+} & =(0,1,-1,-1), \\
\left(D_{6}, D_{7}, D_{8}, S\right) \cdot C_{1,9}^{+} & =(0,-1,1,-1), \\
\left(D_{6}, D_{7}, D_{8}, S\right) \cdot C_{1,10}^{-} & =(1,-1,-1,1) .
\end{aligned}
$$

By (7.21), we then have

$$
D_{\Phi} \cdot C_{1,9}^{+}=D_{\Phi} \cdot C_{1,10}^{-}=0, \quad D_{\Phi} \cdot C_{1,8}^{+}=1 .
$$


These intersection numbers are again consistent with a non-splitting of $F_{\Phi}$, which intersects the split curve $F_{8}$ at the component $C_{1,8}^{+}$. Because $C_{1,8}^{+}$is contained in $S$, so $F_{\Phi}$ must be contained (see table 6).

At weak coupling, this phase has a $5 \mathrm{~d} \mathrm{SU}(2)_{\text {gauge }}+1 \mathbf{F}$ description with flavor symmetry $G_{\mathrm{F}, \mathrm{cl}}=\mathrm{SO}(2) \cong \mathrm{U}(1)$ as well as the $\mathrm{U}(1)_{T}$ symmetry, consistent with no $F_{i}$ fully wrapped. However, by passing to strong coupling, there is a non-trivial enhancement induced by the $\mathrm{U}(1)_{T}$, leading to a non-abelian $\mathrm{SU}(2)_{\mathrm{F}}$ part indicated by $F_{\Phi}$ being wrapped. To preserve rank, we must then have $G_{\mathrm{F}}=\mathrm{SU}(2) \times \mathrm{U}(1)$.

Phase IX. The box graph for phase IX implies the splitting

$$
F_{7} \rightarrow 2 C_{1,8}^{-}+F_{8}+f .
$$

These three curves arrange as

$$
\ldots-F_{6}-F_{8}-C_{1,8}^{-}-f
$$

in order to comply with the intersection numbers $S \cdot F_{7}=S \cdot F_{8}=D_{7} \cdot f=0$, as well as the weights of the curve $C_{1,8}^{-}$:

$$
\left(D_{7}, D_{8}, S\right) \cdot C_{1,8}^{-}=(-1,1,1) .
$$

These intersection numbers in turn determine, as we know from (7.21),

$$
D_{\Phi} \cdot C_{1,8}^{-}=-1
$$

This means that $D_{\Phi}$ contains $C_{1,8}^{-}$, and hence the fiber component $F_{\Phi}$ must split,

$$
F_{\Phi} \rightarrow C_{1,8}^{-}+\Gamma
$$

Since we know $S \cdot F_{\Phi}=0$, we can compute the intersection numbers of the new curve $\Gamma$,

$$
\left(D_{1}, \cdots, D_{8}, D_{\Phi}, S\right) \cdot \Gamma=(0,0,0,0,0,0,1,0,-1,-1) .
$$

Thus $\Gamma \subset S$. Since $\Gamma$ is not a curve appearing in the box graph, it has to be a (multi-)section of the ruling, and hence $\Gamma \cdot S f \geq 1 .{ }^{21}$

On the other hand, $\Gamma$ and $C_{1,8}^{-}$must be also attached due to the splitting (7.31).

Naively, it would appear as if $C_{1,8}^{-}$, which lies outside of $S$, had two different intersection points with each $f$ and $\Gamma$ that are in $S$. This is clearly in violation of $S \cdot C_{1,8}^{-}=1$. The resolution of this puzzle is that the point in which $S$ and $C_{1,8}^{-}$intersect is also an intersection point of $f$ and $\Gamma$ inside $S$. The resulting fiber picture is depicted in table 6 . Since the weak coupling $\mathrm{SU}(2)_{\text {gauge }}$ description has no matter, there is only the topological $\mathrm{U}(1)_{T}$. As $F_{\Phi}$ splits off $C_{1,8}^{-}$which is not wrapped, there is no non-abelian enhancement, and we have $G_{\mathrm{F}}=\mathrm{U}(1)$.

Note that because the $(-1)$-curve $\Gamma$ is not part of the box graph, the gauge theory description of the geometry does not see the transition corresponding to flopping this

\footnotetext{
${ }^{21}$ In the explicit resolution, cf. Part I [2] , one can explicitly show $\Gamma \cdot_{S} f=1$.
} 
curve. Nevertheless, to consistently embed the $\mathrm{SO}(16)_{\mathrm{F}}$ box graph splittings into the affine $E_{8}$ Dynkin diagram requires the existence of this curve, which we can now flop. The resulting Phase XI has no gauge theory description and no flavor symmetry. Obviously, this phase is not visible in the gauge theoretic approach, but nevertheless part of the geometric classification, see $[2,10,12,39]$.

Phase $\mathbf{X}$. The splitting is

$$
F_{8} \rightarrow 2 C_{1,9}^{-}+F_{7}+f
$$

From the intersections,

$$
\left(D_{7}, D_{8}, S\right) \cdot C_{1,9}^{-}=(1,-1,1)
$$

it follows that $C_{1,9}^{-}$is not contained inside the surface $S$. Furthermore, we have $D_{7} \cdot C_{1,9}^{-}=1$ and $D_{7} \cdot f=0$, corresponding to a fiber with

$$
\ldots-F_{6}-F_{7}-C_{1,9}^{-}-f-\ldots
$$

Now, because we have $D_{\Phi} \cdot C_{1,9}^{-}=D_{\Phi} \cdot F_{7}=0, F_{\Phi}$ does not split. However, since $D_{\Phi} \cdot F_{8}=1$, we see that $F_{\Phi}$ must attach to the above chain at the curve $f$ to preserve the intersection number. Then, because $f$ is contained in $S$ as its ruling, $F_{\Phi}$ must as well be a curve in $S$ to satisfy $S \cdot F_{\Phi}=0$. We can see the structure in table 6 .

The wrapped $(-2)$-curve $F_{\Phi}$ gives the $\mathrm{SU}(2)_{\mathrm{F}}$ flavor group in the SCFT limit of the pure $\mathrm{SU}(2)_{\text {gauge }}$ gauge theory. Note that in this case, we see that the surface $S$ does not contain any $(-1)$-curves. This not only explains the absence of any massless charged matter at weak coupling, but also why this phase cannot be further flopped geometrically, i.e., the SCFT does not have any further mass deformations.

\subsection{Classification of rank 2 theories from box graphs}

From the Coulomb branch phases/box graphs of the rank one 5d gauge theories we learned two things: the box graphs give a succinct representation-theoretic description of all the Coulomb branch phases - and thereby characterization of all 5d SCFTs with a weaklycoupled gauge theory description, as well as the mass deformations and RG-flows connecting them. Secondly, the box graphs reconstruct the geometry, in particular curves that are contained in the compact divisors of the M-theory realization. These in turn determine flavor symmetries of the $5 \mathrm{~d}$ UV fixed point theories. The only additional input that is necessary is the embedding of the weakly-coupled flavor symmetry of the marginal theory $G_{\mathrm{BG}}$, which is fixed with one geometric input (in rank one, this is the embedding of $\mathrm{SO}(16)$ into $\widehat{E}_{8}$, which determines how the additional curve $F_{\Phi}$ is attached). This approach also provides a gauge theoretic counterpart to the geometric classification and properties of the rank two 5d SCFTs $[2,10]$. The corresponding marginal theories have weakly-coupled gauge theory descriptions as summarized in Part I, appendix A [2]. 


\subsubsection{Marginal theories and box graphs}

We present all rank two gauge/quiver descriptions, and determine their box graphs and descendant trees in appendix A. Let us briefly summarize the theories here.

The marginal 5d theory arising from the rank two E-string theory on $S^{1}$ has the following weakly coupled gauge theory descriptions:

- $\mathrm{SU}(3)_{\frac{3}{2}}+9 \boldsymbol{F}$,

- $\operatorname{Sp}(2)+8 \boldsymbol{F}+1 \boldsymbol{A} \boldsymbol{S}$

- $5 \boldsymbol{F}-\mathrm{SU}(2)-\mathrm{SU}(2)-2 \boldsymbol{F}$.

The box graphs for these theories are shown in figures 30,31, and 32, respectively.

For the minimal $\left(D_{5}, D_{5}\right)$ conformal matter theory on $S^{1}$, there are also three weakly coupled descriptions of the marginal theory:

- $\mathrm{SU}(3)_{0}+10 \boldsymbol{F}$

- $\operatorname{Sp}(2)+10 \boldsymbol{F}$

- $4 \boldsymbol{F}-\mathrm{SU}(2)-\mathrm{SU}(2)-4 \boldsymbol{F}$.

The box graphs for these, and the descendant theories are shown in figures 33, 34, and 35, respectively.

There are additional 5d marginal gauge theories which act as starting points for RGflows, which are discussed in appendix A. There are a few observations to be made: the tree structure matches that of the geometric transitions/flops as well as CFD-transitions for rank two SCFTs. Furthermore, the theories match precisely with those that are known to have a gauge theory description. This is strong evidence that the flavor-equivalence classes of box graphs captures these theories correctly.

To make further use of these Coulomb branch descriptions, we need to add, much like in the rank one case, the information about the embedding of the flavor symmetry of the marginal theory into the $6 \mathrm{~d}$ flavor symmetry. Once we have supplemented the box graphs with this information, the superconformal flavor symmetries of all descendants can be read off as well — this is already included in the figures in appendix A.

\subsubsection{Phases to fibers}

We exemplify this now by studying the rank two E-string and $\left(D_{5}, D_{5}\right)$ minimal conformal matter theories, that have both a description in terms of a marginal SU(3) gauge theory.

Recall that geometrically, the theories descending from the rank two E-string are obtained from M-theory on an elliptically fibered Calabi-Yau threefold, with a non-minimal singularity from an $\left(E_{8}, \mathrm{SU}(2)\right)$ collision. Let us denote the affine $E_{8}$ fiber components and corresponding non-compact divisors by $F_{i}^{E_{8}} \hookrightarrow D_{i}^{E_{8}}, i=1, \cdots, 9$ as shown in (7.36), ${ }^{22}$ and the two $\mathrm{SU}(2)$ components by $F_{i}^{\mathrm{SU}(2)} \hookrightarrow D_{i}^{\mathrm{SU}(2)}, i=1,2$.

\footnotetext{
${ }^{22}$ We choose this slightly non-standard enumeration as this is more natural when identifying the embedding of the $\mathrm{U}(9)$ flavor symmetry.
} 


\begin{tabular}{|c|c|c|c|c|c|c|c|c|c|c|c|c|c|c|c|c|c|}
\hline 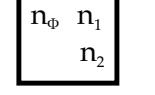 & \multicolumn{3}{|c|}{$\alpha_{1}$} & \multicolumn{2}{|c|}{$\alpha_{2}$} & \multicolumn{2}{|c|}{$\alpha_{3}$} & \multicolumn{2}{|c|}{$\alpha_{4}$} & \multicolumn{2}{|c|}{$\alpha_{5}$} & \multicolumn{2}{|c|}{$\alpha_{6}$} & \multicolumn{2}{|c|}{$\alpha_{7}$} & \multicolumn{2}{|c|}{$\alpha_{8}$} \\
\hline \multirow{3}{*}{$\begin{array}{l}\alpha_{2}^{\text {gauge }} \\
\alpha_{1}^{\text {gauge }}\end{array}$} & -1 & $\begin{array}{c}0 \\
-1\end{array}$ & -1 & -1 & -1 & & -1 & $\begin{array}{r}0 \\
-1\end{array}$ & -1 & -1 & & $\begin{array}{c}0 \\
-1\end{array}$ & & $\begin{array}{c}0 \\
-1\end{array}$ & & $\begin{array}{c}0 \\
-1\end{array}$ & $\begin{array}{ll}0 & 0 \\
& -1\end{array}$ \\
\hline & 0 & $\begin{array}{l}0 \\
0\end{array}$ & 0 & 0 & 0 & 0 & 0 & $\begin{array}{l}0 \\
0\end{array}$ & 0 & 0 & & $\begin{array}{l}0 \\
0\end{array}$ & & $\begin{array}{l}0 \\
0\end{array}$ & & $\begin{array}{l}0 \\
0\end{array}$ & $\begin{array}{ll}1 & 0 \\
& 0\end{array}$ \\
\hline & 0 & $\begin{array}{l}1 \\
0\end{array}$ & 0 & 0 & 0 & $\begin{array}{l}1 \\
0\end{array}$ & 0 & $\begin{array}{l}1 \\
0\end{array}$ & 0 & 1 & & $\begin{array}{l}1 \\
0\end{array}$ & & $\begin{array}{l}1 \\
0\end{array}$ & 1 & $\begin{array}{l}1 \\
0\end{array}$ & $\begin{array}{ll}1 & 1 \\
& 0\end{array}$ \\
\hline
\end{tabular}

Figure 26. The pairings $n_{l}=\left\langle\alpha_{l}^{\vee}, L\right\rangle$ between the $(\mathbf{3}, \mathbf{9})$-weights $L$ and the coroots $\alpha_{l}^{\vee}$ that are not part of $\mathrm{U}(9)_{\mathrm{BG}}$. For any decoration of this box graph, these numbers encode via (7.4) the geometric intersections with additional non-compact divisors $D_{\Phi_{l}}$ over the affine $E_{8}$ and $\mathrm{SU}(2)$ fibers. Specifically, $n_{\Phi}$ denotes the pairing with the coroot $D_{\Phi}$ (the additional $E_{8}$ coroot), and $n_{1,2}$ those with $D_{1,2}^{\mathrm{SU}(2)}$.

The $5 \mathrm{~d}$ theories descending from circle compactifications of this $6 \mathrm{~d}$ model have rank two. From an explicit blow-up resolution (see appendix C.1) one can read off the three different 5d gauge theory descriptions listed above. In the following we will discuss the fiber reconstruction from the box graphs from the SU(3) gauge theory description.

For the rank two E-string on $S^{1}$, the marginal theory is $\mathrm{SU}(3)+9 \boldsymbol{F}$, and the descendants are characterized in terms of box graphs for $\mathrm{SU}(3) \times \mathrm{U}(9)_{\mathrm{BG}}$. There is essentially one unique way to embed the eight roots $F_{i}$ of $\mathrm{SU}(9) \subset \mathrm{U}(9)$ into the codimension one fibers of $E_{8} \times \mathrm{SU}(2)$, namely, into the connected chain of eight nodes inside the affine $E_{8}$ diagram. We fix the ambiguity of ordering by the identification:

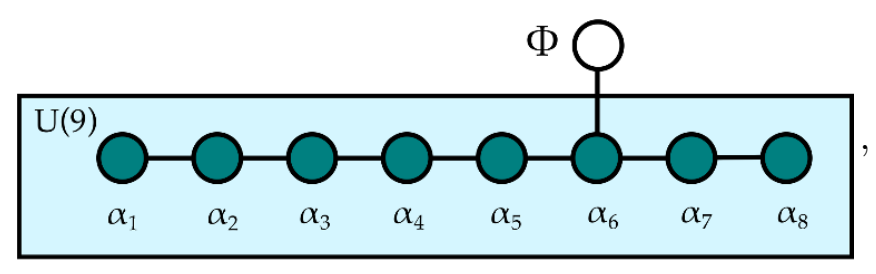

where $F_{\Phi} \equiv F_{9}^{E_{8}}$ is the additional node that the gauge theory phase does not capture. This leaves codimension one fibers $F_{\Phi}^{E_{8}} \hookrightarrow D_{\Phi}^{E_{8}}$ and $F_{1,2}^{\mathrm{SU}(2)} \hookrightarrow D_{1,2}^{\mathrm{SU}(2)}$ out of the box graphs, which may be interpreted as additional coroots $\Phi^{\vee} \equiv \Phi_{0}^{\vee}$ and $\Phi_{1,2}^{\vee}$, respectively. As in the rank one cases, we first determine the intersections $\left\langle\Phi_{l}^{\vee}, L_{m}\right\rangle$ of the box graph weights $L_{m}$. We do this in the explicit resolution detailed in appendix C.1. From the intersection numbers (C.3) between the extremal curves associated with these sign assignments and the divisors $D_{\Phi}^{E_{8}}$ and $D_{1,2}^{\mathrm{SU}(2)}$, we can then infer the intersections of all the curves. We collect this information in the representation graph in figure 26 .

To clarify the process, we provide three concrete examples in figure 27 . The fibers are precisely the ones we discussed in Part I [2] from a direct resolution computation of non-minimal singularity. 


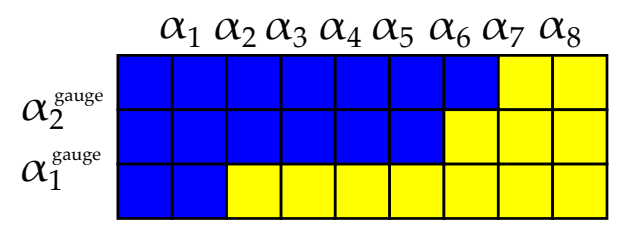

(a) $\mathrm{SU}(3) \times \mathrm{U}(9)$ Box Graph.

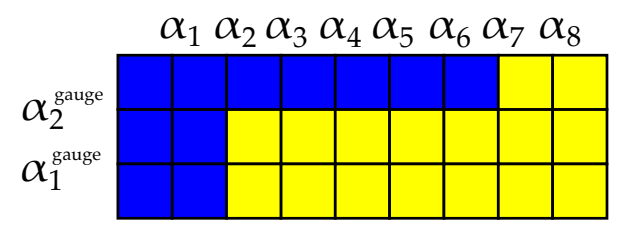

(c) $\mathrm{SU}(3) \times \mathrm{U}(9)$ Box Graph.

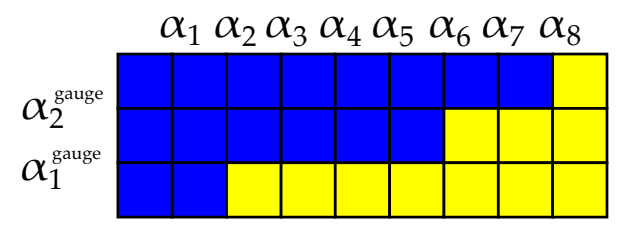

(e) $\mathrm{SU}(3) \times \mathrm{U}(9)$ Box Graph.

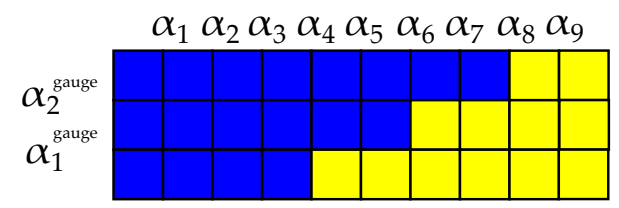

(g) $\mathrm{SU}(3) \times \mathrm{U}(10)$ Box Graph.

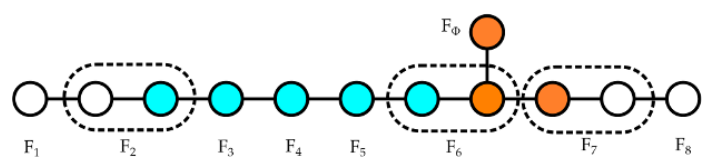

(b) Rank 2 E-string codim 2 fiber for (a) $\left(\mathrm{BU}_{1}^{\left(E_{8}, \mathrm{SU}(2)\right)}\right)$.

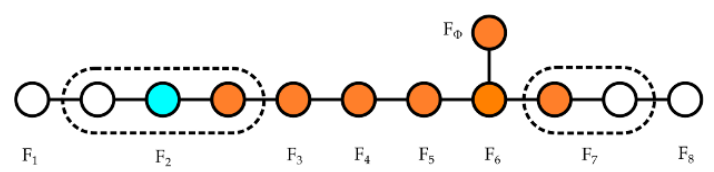

(d) Rank 2 E-string codim 2 fiber for (c) $\left(\mathrm{BU}_{2}^{\left(E_{8}, \mathrm{SU}(2)\right)}\right)$.

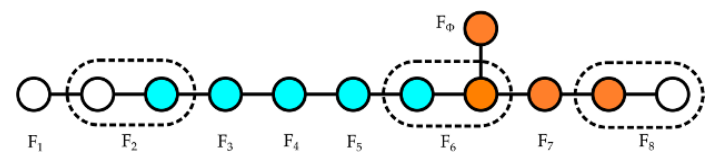

(f) Rank 2 E-string Codim 2 fiber for (e) $\left(\mathrm{BU}_{3}^{\left(E_{8}, \mathrm{SU}(2)\right)}\right)$.

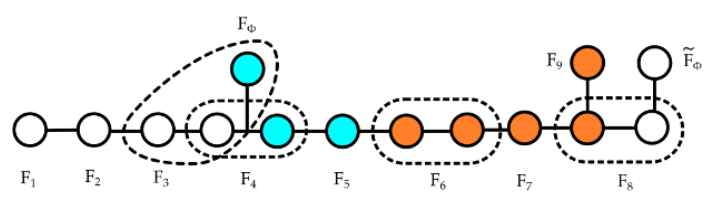

(h) $\left(D_{5}, D_{5}\right)$ Codim 2 fiber for $(\mathrm{g})\left(\mathrm{BU}_{1}^{\left(D_{10}, I_{1}\right)}\right)$.

Figure 27. Examples, (a) to (f), of box graphs and the associated codimension two fibers for $\mathrm{SU}(3)_{\text {gauge }} \times \mathrm{U}(9)_{\mathrm{BG}}$ phases of the rank two E-string, as well as one example, (g) and $(\mathrm{h})$, for $\left(D_{5}, D_{5}\right)$ conformal matter; these examples have appeared in Part I, [2], in terms of explicit resolutions. Note that (a) and (c) are in the same flavor equivalence class. The roots for $G_{\mathrm{BG}}$ are denoted $\alpha_{i}$ and associated curves $F_{i}$. The roots of the gauge group are $\alpha_{i}^{\text {gauge }}$, which are dual to the compact surfaces $S_{i}, i=1,2$. The colors cyan/orange on the r.h.s. indicate which codimension one curves $F_{i}$ are contained in which $S_{i}$. Note that for the rank two E-string the $\mathrm{SU}(2)$ part of the fiber also splits, but does not contribute in these examples to the flavor symmetry and we omit to draw it.

We can repeat the exercise for the marginal $\mathrm{SU}(3)+10 \boldsymbol{F}$ gauge description of the $S^{1}$-reduction of the $\left(D_{5}, D_{5}\right)$ minimal conformal matter theory. The box graphs for all descendants are shown in figure 33. To reconstruct the fiber in these cases, we consider the embedding of the classical flavor symmetry $\mathrm{U}(10)_{\mathrm{BG}}$ into $6 \mathrm{~d}$ superconformal flavor symmetry, i.e., the affine $\mathrm{SO}(20)$ Dynkin diagram

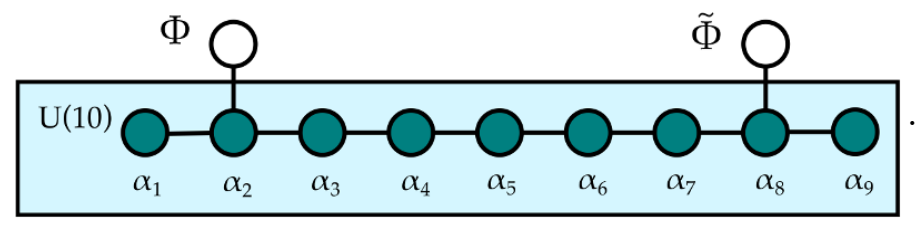


Let us denote the curves associated to the "extra" nodes of the affine $\mathrm{SO}(20)$ codimension one fiber as $F_{\Phi} \hookrightarrow D_{\Phi}$ and $F_{\widetilde{\Phi}} \hookrightarrow D_{\widetilde{\Phi}}$, where we fix the ordering by

$$
D_{F_{\Phi}} \cdot F_{2}=D_{2} \cdot F_{\Phi}=1, \quad D_{\widetilde{\Phi}} \cdot F_{8}=D_{8} \cdot F_{\widetilde{\Phi}}=1,
$$

and zero intersection with all other components of the affine $\mathrm{SO}(20)$ fiber. These corresponds to roots $\Phi, \widetilde{\Phi}$ that do not feature in the flavor group $\mathrm{U}(10)_{\mathrm{BG}}$ of the marginal $\mathrm{SU}(3)_{\text {gauge }}$ description. From a concrete resolution, one can determine the corresponding coroots having the following pairings with the $(\mathbf{3}, \mathbf{1 0})$ weights $L_{i, j}$ in the top and bottom row of a decorated $\mathrm{SU}(3)_{\text {gauge }} \times \mathrm{U}(10)_{\mathrm{BG}}$ box graphs:

$$
\left(\begin{array}{c}
\left\langle\Phi^{\vee}, L_{1, j}^{ \pm}\right\rangle \\
\left\langle\widetilde{\Phi}^{\vee}, L_{1, j}^{ \pm}\right\rangle
\end{array}\right)=\mp\left(\begin{array}{llllllllll}
1 & 1 & 0 & 0 & 0 & 0 & 0 & 0 & 0 & 0 \\
1 & 1 & 1 & 1 & 1 & 1 & 1 & 1 & 0 & 0
\end{array}\right),
$$

and

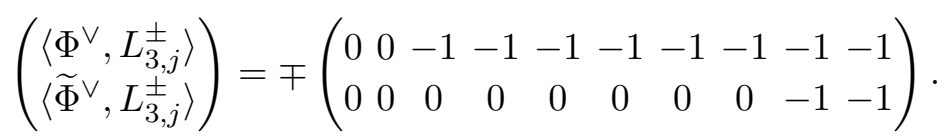

Using the same methods as for the $\mathrm{SU}(3)_{\text {gauge }} \times \mathrm{U}(9)_{\mathrm{BG}}$ phases above, one can determine with this information whether the fibers $F_{\Phi}, F_{\widetilde{\Phi}}$ are contained in the compact surface for any decorated $\mathrm{SU}(3)_{\text {gauge }} \times \mathrm{U}(10)_{\mathrm{BG}}$ box graph. An example is shown in figures $(\mathrm{g})$ and (h) of 27.

\section{SCFTs/CFDs from box graphs}

As we have already argued in section 3, the box graphs can be condensed into so-called flavor equivalence classes. In this section, we show explicitly how this reduction of redundant information is mimicked in the (BG-)CFD representation of the geometry.

To begin with, let us first recall from section 3 that the flavor equivalence classes are characterized by a set $\alpha_{j}^{\mathrm{BG}}$ of flavor roots which are contained in the combined splitting (3.2) of gauge roots in this equivalence class. This splitting can be inferred from the reduced box graphs. Geometrically, these flavor roots correspond to the set of codimension one curves $F_{i}$ which are contained in the sum of all the rulings $f_{j}$ which split according to (7.5). Since flops between flavor equivalent phases by definition do not change this overall splitting, the particular set of flavor roots remain parts of the rulings on $\mathcal{S}$ in all phases, and hence collapse in the non-abelian gauge enhancing limit, giving rise to the classical flavor symmetry $G_{\mathrm{F}, \mathrm{cl}}$ of this equivalence class. Moreover, the set of (-1)-curves corresponding to the F-extremal weights also remain, by definition, invariants within a flavor equivalence class. Note that in some phases, the curve may be reducible; however again by definition - there always exists a phase in which the curve does not split. Thus, the BG-CFDs precisely correspond to the intersection pattern of the curves associated with the F-extremal weights and the flavor roots contained in the splitting of gauge roots.

The embedding of the BG-CFDs into the full CFD also played an important role in our discussion of constraining possible gauge descriptions of SCFTs. Again, this is based on the underlying geometry and intersection theory. In the following, we will show how 
the reduced box graphs, that specify the BG-CFDs, also determine superconformal flavor symmetry once we specify the attachments of the "missing" codimension one components $F_{\Phi_{l}} \hookrightarrow D_{\Phi_{l}}$.

\subsection{Box graphs to superconformal flavor symmetry and CFDs}

The argument follows the same logic as in the previous section, where we have discussed in detail how to determine the explicit fiber structure from a decorated box graph. Namely, we need to clarify if the extra roots $\Phi_{l}$ are part of the CFD or not. This requires the minimal geometric input in form of the pairings

$$
\mathcal{E}_{l, n}=\left\langle\Phi_{l}^{\vee}, L_{n}\right\rangle
$$

between the extra coroots $\Phi_{l}^{\vee}$ and the F-extremal weights $L_{n}^{\epsilon_{n}}$, which translates into geometric intersections between F-extremal curves $C_{n}^{\epsilon_{n}}$ and divisors $D_{\Phi_{l}}$. At the level of flavor equivalence classes, consistency of intersection numbers and the reduced box graphs themselves implies the following rules on how to attach the node $\Phi_{l}$ to the BG-CFD:

1. If $-\epsilon_{n} \mathcal{E}_{l, n}<0$ for an F-extremal weight $L_{n}^{\epsilon_{n}}$, that is not contained in the BG-CFD, then $\Phi_{l}$ is not part of the CFD. This is because the inequality implies $\Phi_{l} \rightarrow L_{n}^{\epsilon_{n}}+\cdots$, and $L_{n}^{\epsilon_{n}}$ is not in the BG-CFD.

2. If $-\epsilon_{n} \mathcal{E}_{l, n} \geq 0$ for all $L_{n}^{\epsilon_{n}}$ that are not part of the BG-CFD, but there exists one $L_{m}^{\epsilon_{m}}$ that is a $(-1)$-vertex in the BG-CFD, such that $-\epsilon_{m} \mathcal{E}_{l, m}>0$, then $F_{\Phi_{l}}$ has a non-empty intersection with a curve in the CFD. Because it does not split into anything outside the BG-CFD, $\Phi_{l}$ is a $(-2)$-vertex inside the full CFD.

3. If $-\epsilon_{n} \mathcal{E}_{l, n} \geq 0$ for all F-extremal weights $L_{n}^{\epsilon_{n}}$ that are not in the BG-CFD, and $-\epsilon_{m} \mathcal{E}_{l, m} \leq 0$ for all F-extremal weights $L_{m}^{\epsilon_{m}}$ that are part of the BG-CFD, with at least one $m$ such that $-\epsilon_{m} \mathcal{E}_{l, m}<0$, then $\Phi_{l}$ becomes reducible and splits into weights/roots in the BG-CFD, and thereby has to also be contained as a $(-2)$ vertex.

4. Finally, if $\mathcal{E}_{l, m}=0$ for all F-extremal weights $L_{m}^{\epsilon_{m}}$ in the BG-CFD, then $\Phi_{l}$ is a (-2)vertex of the CFD if and only if there is a $(-2)$-vertex $F_{\ell}$ in the BG-CFD $\ell$ such that $D_{\Phi_{l}} \cdot F_{\ell}>0$.

With these rules we can reconstruct a CFD, which captures the non-abelian part of the superconformal flavor symmetry, from which it is then easy to infer the abelian factors from the classical flavor symmetry and the number of instanton U(1)s, see (7.1).

Note however, that the CFDs we construct in this way - which we will refer to as reduced CFDs - are generically sub-graphs of the full CFDs that we defined in [1] and derived from the geometry in [2]. There are $(-1)$-vertices corresponding to non-perturbative states of the gauge description associated with the BG-CFD, as well as unmarked vertices with $n_{i} \geq 0$, which cannot be reconstructed in this way. What we capture using the present gauge theoretic approach is the superconformal flavor symmetry (encoded in the $(-2)$-vertices) and tree-structure (captured by the $(-1)$-vertices, upon which we can apply 
the standard CFD-transitions) of the reduced CFDs, which have the gauge description of the chosen BG-CFD. However, as we have discussed in section 6, one can access other branches of the descendant tree by passing to a dual gauge description, and consider the BG-CFDs of such theories.

In summary: the reduced CFDs are constructed from the flavor-equivalence classes of $G_{\text {gauge }} \times G_{\mathrm{BG}}$ box graphs, in conjunction with minimal input from the geometry, which

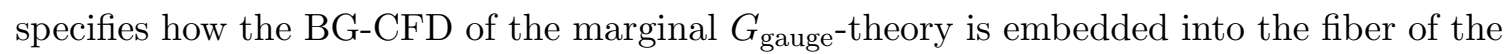
elliptic model that describes the marginal theory. The reduced CFDs contain

- marked subgraph (and thereby the Dynkin diagram of the superconformal flavor symmetry) of the full CFD,

- $(-1)$ vertices, which in the full CFD have an interpretation as matter hypermultiplets charged under $G_{\text {gauge }}$.

It does not contain those $(-1)$ curves (and higher self-intersection curves), which transition, when flopped, to a geometry without a compatible ruling, and thus to an SCFT that does not have a weakly coupled $G_{\text {gauge }}$ description.

\subsection{Rank two CFDs from box graphs}

We now use the above approach to determine for all rank two theories the superconformal flavor symmetry, starting from the box graphs and the minimal information from the fibers. The results are summarized in appendix A.

To start with, let us again consider the $\mathrm{SU}(3)_{\text {gauge }} \times \mathrm{U}(9)_{\mathrm{BG}}$ example we studied in section 3.2, and shown in figure 28 (see also 27, (a) and (c)). On the right hand side of figure 28, there are three box graphs that are in the same flavor equivalence class. The reduced box graph for this equivalence class is given by simply deleting the middle row, as shown on the left hand side of figure 28. The splitting dictated for the whole flavor equivalence class is given by the F-extremal weights ${ }^{23} L_{1,7}^{+}, L_{1,8}^{-}, L_{3,2}^{+}, L_{3,3}^{-}$, and the following roots become reducible

$$
\begin{aligned}
& F_{2} \rightarrow L_{3,2}^{+}+L_{3,3}^{-} \\
& F_{7} \rightarrow L_{1,7}^{+}+L_{1,8}^{-} .
\end{aligned}
$$

Note that in the full box graphs on the r.h.s. of figure 28, the F-extremal weights are not always extremal (can be flopped in the box graph). An example is in model (b) the weight $L_{1,7}^{+}$and in (c) the weight $L_{3,3}^{-}$. We will see momentarily that this is however immaterial in determining the flavor symmetry.

Recall that the $\mathrm{SU}(3)_{\text {gauge }} \times \mathrm{U}(9)_{\mathrm{BG}}$ box graph provide a gauge theory description of the rank two E-string (realized by a $\left(E_{8}, \mathrm{SU}(2)\right)$ collision of singularities), where the $\mathrm{SU}(9)_{\mathrm{BG}} \subset \mathrm{U}(9)_{\mathrm{BG}}$ fully embedded inside the affine $E_{8}$ Dynkin diagram as in (7.36).

The BG-CFD is already determined for this flavor equivalence class in figure 5 . In particular the CFD contains the roots $\alpha_{i}, i=3, \ldots, 6$ as $(-2)$-vertices and, since

\footnotetext{
${ }^{23}$ We use the same labeling for weights as in figure 6 .
} 

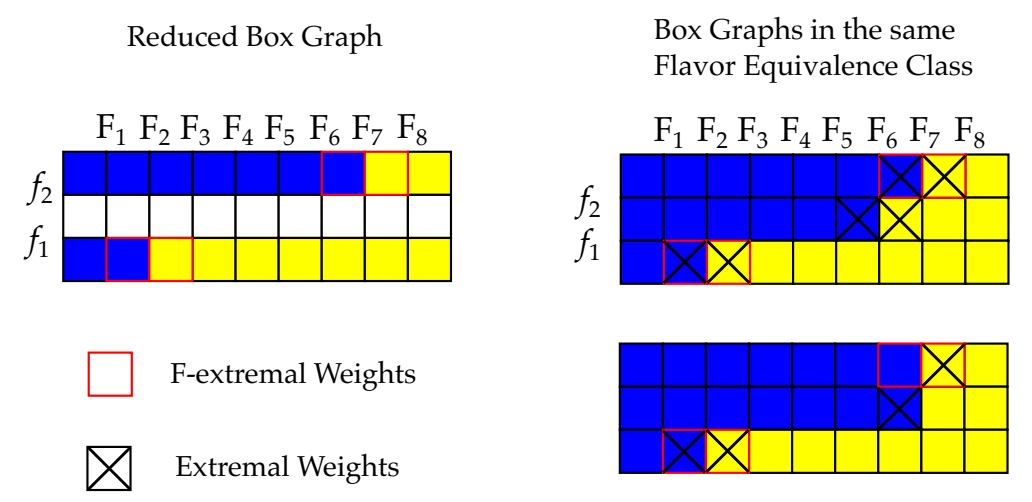

(a)

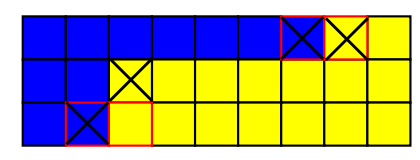

(c)

Figure 28. An example of a reduced box graph (r.h.s.) and elements in the same flavor equivalence class (box graphs shown on the right) for $\mathrm{SU}(3) \times \mathrm{U}(9)$. The F-extremal curves are marked in red, the extremal curves of the box graphs are marked by a cross. This shows that F-extremal curves are not necessarily always extremal for every box graph of the flavor equivalence class. The key is that changing the sign assignment of the F-extremal curves results in a different SCFT and superconformal flavor symmetry. The box graphs in a given flavor equivalence class are all distinct gauge theory descriptions that have the same UV fixed point.

$\left\langle\alpha_{3}^{\vee}, L_{3,3}\right\rangle=-\left\langle\alpha_{6}^{\vee}, L_{1,7}\right\rangle=-1$, the two weights $L_{3,3}^{+}$and $L_{1,7}^{-}$as $(-1)$-vertices. We next need to determine whether there are any additional vertices $\Phi_{l}$ from the geometry.

For this note that we determined already the pairings $\mathcal{E}_{l, n}$ between the weights $L_{n}$ and the additional roots $\Phi_{l}$ for this case in figure 26. These were determined from the marginal resolution geometry. Denoting by $\Phi$ and $\Phi_{l}, l=1,2$ the roots associated with the three extra nodes, first observe that

$$
\left\langle\Phi^{\vee}, L_{i, j}\right\rangle=0,
$$

for all F-extremal weights $L_{i, j}$ in the flavor equivalence class. Furthermore, $D_{\Phi} \cdot F_{6}=1$. As $F_{6}$ is part of the BG-CFD, $\Phi$ is a $(-2)$-vertex in the CFD as well, and will contribute thereby to $G_{\mathrm{F}}$.

To determine whether the roots $\Phi_{1,2}$ of the affine $\mathrm{SU}(2)$ are part of the CFD, note that

$$
\left\langle\Phi_{1}^{\vee}, L_{3,2}\right\rangle=1 \text {. }
$$

Since in this reduced box graph, the sign of $L_{3,2}$ (which is not part of the BG-CFD) is + , rule 1. above implies that $\Phi_{1}$ not part of the CFD either. Likewise,

$$
\left\langle\Phi_{2}^{\vee}, L_{1,8}\right\rangle=-1
$$

which, together with the sign (-) and the fact that $L_{1,8}$ is not in the BG-CFD, implies that $\Phi_{2}$ does not contribute, either. What we obtain is the CFD shown in figure 29.

To conclude this example, the non-abelian part of the superconformal flavor symmetry is $G_{\mathrm{F}, \mathrm{na}}=\mathrm{SU}(6)$. For this particular flavor equivalence class, the associated $5 \mathrm{~d}$ effective gauge theory is $\mathrm{SU}(3)_{\text {gauge }}+5 \boldsymbol{F}$, so its total global symmetry (at weak coupling) is 


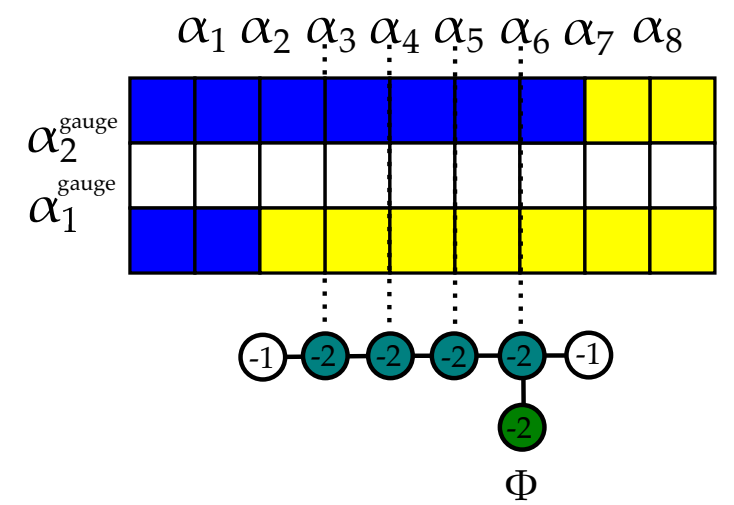

Figure 29. The BG-CFD of the $\mathrm{SU}(3)_{\text {gauge }}$ theory presented in figure 5 , including the additional node, inferred from the geometric data. The non-abelian part of the superconformal flavor symmetry is determined by the $(-2)$ (marked/green) vertices, and is $\mathrm{SU}(6)$. The mass deformations that lead

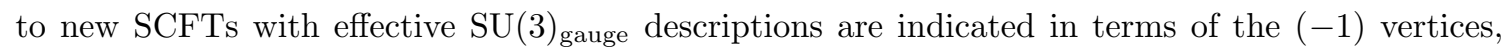
to which we can apply CFD-transitions. While it is not the full CFD, this reduced CFD, contains the same marked subgraph, and the $(-1)$ vertices of the reduced graph are also present in the full CFD, which are the central ingredients for the flavor symmetry and the descendant tree.

$\mathrm{U}(5) \times \mathrm{U}(1)_{I}$. To match the total rank, the superconformal flavor symmetry then must be

$$
G_{\mathrm{F}}=\mathrm{SU}(6) \times \mathrm{U}(1)
$$

We can repeat this process for all rank two theories and determine the flavor equivalence classes, associated BG-CFDs, and the reduced CFDs. The results are presented in appendix A. Note that the reduced CFDs contain the full non-abelian part of the flavor symmetry, as above, but may not contain information about additional non-flavor curves. These are therefore sub-graphs of the CFDs, which however contain the complete marked subgraph of the CFD. Furthermore, the $(-1)$ vertices in the reduced CFD are also part of the full CFD, and the resulting descendant tree is therefore a subtree.

\section{Conclusions and outlook}

In this series of papers, the "appetizer" [1], Part I [2], and the present Part II, we made the case that 5d SCFTs which descend from 6d SCFTs by circle compactifications plus mass deformations have a concise description in terms of graphs, the so-called CFDs. These graphs encode some of the salient physical properties of these superconformal field theories:

- They tell us about the network structure of descendant SCFTs from a given $5 \mathrm{~d}$ marginal theory.

- The marked vertices of a CFD form subgraphs that are Dynkin diagrams, which encode the strongly-coupled flavor symmetry $G_{\mathrm{F}}$ of the UV fixed point that the CFD characterizes. 
- The spectrum of BPS states (Gopakumar-Vafa invariants) is computed by taking suitable combinations of the vertices (i.e., curves). Intersections with divisors associated to the marked subgraph graphs determines the representation under $G_{\mathrm{F}}$.

- They constrain the possible weakly coupled gauge theory or quiver descriptions for the associated SCFT.

- They predict dualities among these weakly coupled descriptions.

In summary, the CFDs seem to crystallize some of the important features of 5d SCFTs!

This approach is particularly powerful when applied to theories that descend from $6 \mathrm{~d}$ models whose geometric description has a known construction in terms of a fully singular Tate or Weierstrass model. This was important in order to compute the CFD of the marginal theory - from which all the above properties of the descendants can be determined in a combinatorial fashion. Examples of such theories are all the minimal conformal matter theories, as well as some lower rank theories with automorphisms (e.g., those that occur in the rank two classification $[10,26])$. It is in these instances that we can derive the CFDs and substantiate all claims regarding weakly coupled descriptions, and dualities among these, by performing a complementary geometric computation - a class of examples where these the details were worked out are the $\left(E_{6}, E_{6}\right)$ and $\left(E_{7}, \mathrm{SO}(7)\right)$ minimal conformal matter theories. This geometric confirmation provides backing for other setups, where the geometric computations become less feasible. Some dualities appeared very recently in [71] and it would be interesting to study the relation with the dualities presented in the present paper.

In particular, there are 6d SCFTs which do not have a known description in terms of a singular Weierstrass model. Specifically, $5 \mathrm{~d}$ marginal theories with known $6 \mathrm{~d}$ tensor branch descriptions, e.g., $\mathrm{SU}(N)+2 \boldsymbol{A} \boldsymbol{S}+8 \boldsymbol{F}$ for $N>5$, as well as their descendants, were studied using five-brane webs. In these cases we can "bootstrap" the marginal CFD by using the constraints of known gauge theory descriptions including their superconformal flavor enhancements. Perhaps most interestingly, the resulting marginal CFD in turn predicts new branches of the descendant tree, which would indicate a yet unknown sequence of SCFT descendants with different gauge descriptions. Thus, combining the requirement of the embedding of the gauge theoretic BG-CFDs, the known flavor enhancements for parts of the descendant tree, and the constraint that these all descend from a single marginal CFD by applying the CFD-transition rules, results in new predictions for these theories. It would clearly be very exciting to test these predictions either by constructing the associated Weierstrass models or by alternative methods such as a five-brane web realization.

As already alluded to in the conclusions to Part I [2], the next step in the program to determine all 5d SCFTs that descend from $6 \mathrm{~d}$ is to develop a gluing procedure for CFDs. Similar to the classification in 6d, where the most general theory is built out of a generalized quiver based on a small set of building blocks (the flavor nodes as well as non-Higgsable clusters), a similar gluing is expected to exist in $5 \mathrm{~d}$. Given the fundamental role that the CFDs seem to play, it is very natural to expect them to be (part of) the building blocks from which the most general 5d SCFT is glued. This will be investigated in the future. 


\section{Acknowledgments}

We thank C. Closset, H. Hayashi, J.J. Heckman, M. Weidner, K. Yonekura, and G. Zafrir for discussions. FA, SSN and YNW are supported by the ERC Consolidator Grant number 682608 "Higgs bundles: Supersymmetric Gauge Theories and Geometry (HIGGSBNDL)", CL by NSF CAREER grant PHY-1756996, LL by DOE Award DE-SC0013528Y. FA also thanks the 2019 Summer Workshop at the Simons Center for Geometry and Physics for hospitality during the completion of this work. LL thanks the ITP Heidelberg for hospitality during the completion of this work. SSN is grateful to the CERN Theory Group for hospitality during the course of this work.

\section{A Coulomb branch and reduced CFDs for rank two 5d SCFTs}

This appendix summarizes the box graphs (and flavor-equivalence classes of box graphs) for the rank two 5d SCFTs, which have a weakly coupled gauge theory description. We also note down in each case the reduced CFDs, which were obtained by using minimal input from the resolution geometry to reconstruct the fiber from the box graphs, as explained in section 8 .

\section{A.1 Descendants of rank 2 E-string and $\left(D_{5}, D_{5}\right)$ conformal matter}

For the rank two E-string on $S^{1}$, these are shown in figure 30, 31, 32. For minimal $\left(D_{5}, D_{5}\right)$ conformal matter, they are in figure $33,34,35$.

Each of these theories will be specified by a gauge theory/quiver with a rank two gauge group together with some matter transforming under some flavor group. In the main text we provide a detailed description of the $\mathrm{SU}(3)$ gauge theory description of the rank two E-string in section 3.2. Furthermore we will specify the reduced CFD: as explained in section 8 , we can reconstruct the relevant parts of the fiber from the box graphs and flavorequivalence classes, once we specify the embedding into the fiber of the marginal theory. From this we can further derive the marked vertices of the CFD, i.e., the subgraph that encodes that flavor symmetry at the UV fixed point, as well as the (-1)-vertices, which correspond to decoupling hypermultiplet matter. Note that in general this determines only a sub-graph of the full CFD, and may miss curves with self-intersection number $\geq 0$ or (-1)curves, which do not correspond to hypermultiplets of the chosen gauge description. An example is for instance the rank one CFDs, which have 10 descendants from the marginal theory, from which only 9 have an $\mathrm{SU}(2)$ gauge description. The complete CFDs that are derived from the geometry capture all these descendants, irrespective of whether they admit a weakly coupled description. However, the reduced CFDs that are constructed based on a given gauge theory description, only capture in general a subset. Using the methods in section 8 we can however determine the full superconformal flavor symmetry. 


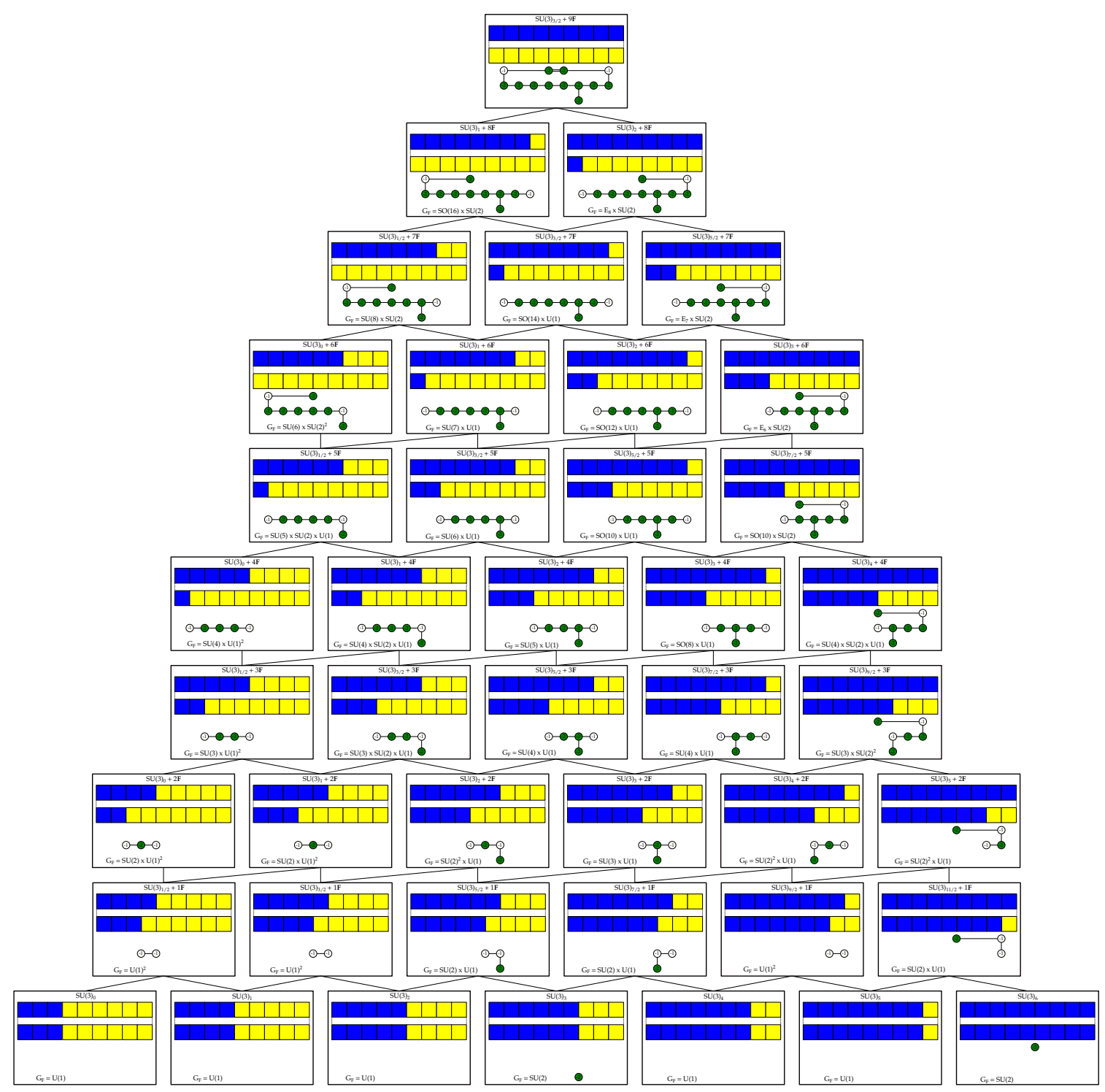

Figure 30. Rank two E-string: the marginal 5 d gauge theory description as $\mathrm{SU}(3)_{3 / 2}+9 \boldsymbol{F}$. The flavor-equivalence classes of this marginal theory can be written in terms of box graphs, and each box graph corresponds to a descendant $5 \mathrm{~d}$ gauge theory. All of the descendant theories have $5 \mathrm{~d}$ superconformal fixed points. Below the box graph equivalence classes we show the reduced CFDs which encode the superconformal flavor symmetry.

\section{A.2 $\mathrm{SU}(3)$ on a (-1)-curve with 12 hypermultiplets}

The marginal theory has three gauge theory descriptions

- $\mathrm{SU}(3)_{4}+6 \boldsymbol{F}$

- $\operatorname{Sp}(2)+2 \boldsymbol{A} \boldsymbol{S}+4 \boldsymbol{F}$

- $G_{2}+6 \boldsymbol{F}$.

The box graphs and descendants for these are shown in figures 36,37 , and 38 . 


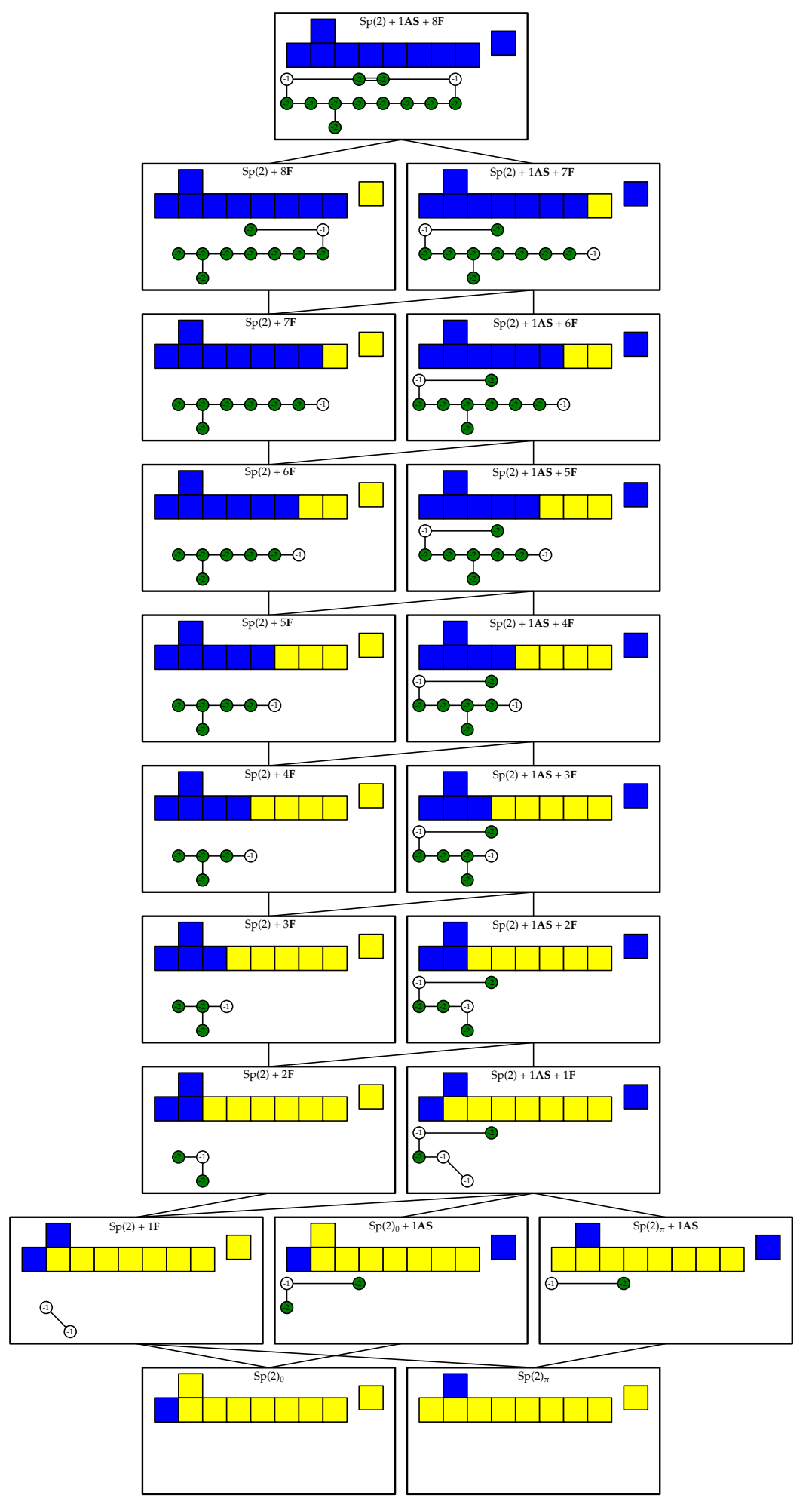

Figure 31. Rank two E-string with the marginal gauge theory described as $\mathrm{Sp}(2)+8 \boldsymbol{F}+1 \boldsymbol{A S}$. The figure shows the tree of descendant theories, together with their superconformal flavor symmetry, $G_{\mathrm{F}}$. We furthermore specify the reduced CFDs, which encode the flavor symmetry and the $(-1)$ vertices that correspond to hypermultiplet matter in the $\mathrm{Sp}(2)$ description. 


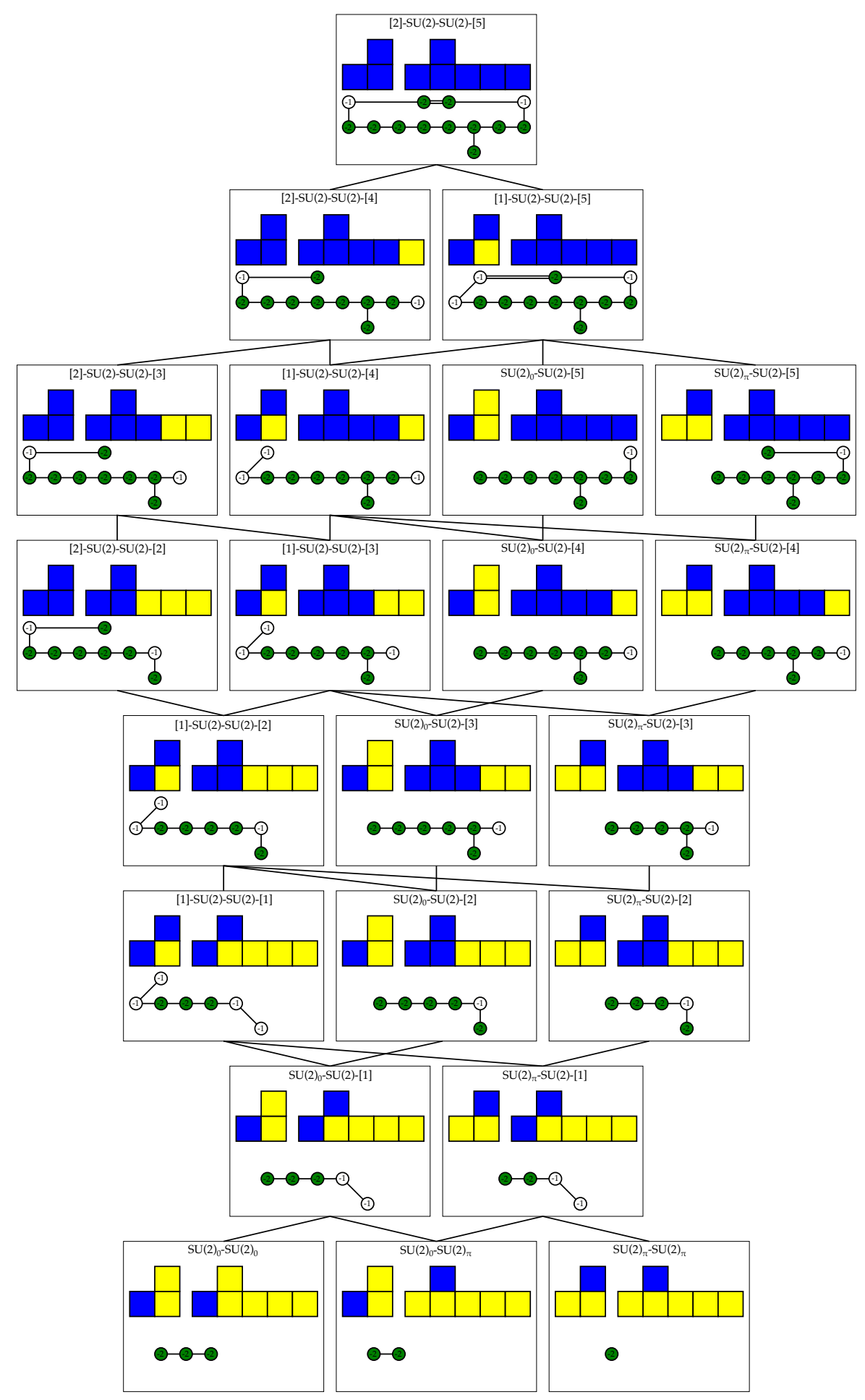

Figure 32. Rank two E-string: descendants of the marginal theory $5 \boldsymbol{F}-\mathrm{SU}(2)-\mathrm{SU}(2)-2 \boldsymbol{F}$, with their flavor-equivalence class of Coulomb phases of $(\mathrm{SU}(2) \times \mathrm{SU}(2))_{\text {gauge }} \times(\mathrm{SO}(10) \times \mathrm{SO}(4))_{\mathrm{BG}}$. In addition we also note the reduced CFDs. 


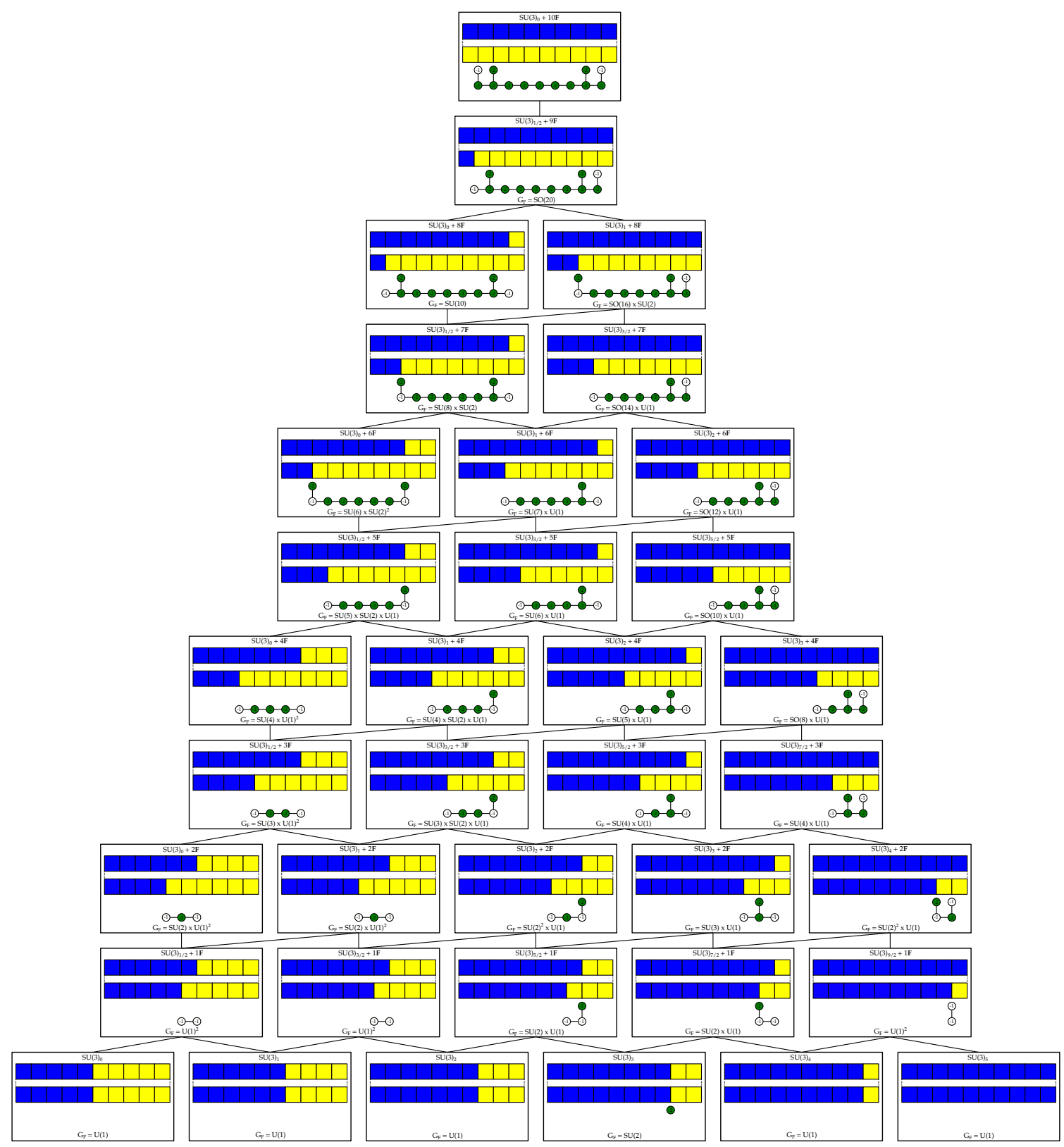

Figure 33. $\left(D_{5}, D_{5}\right)$ Conformal Matter: 5 d marginal gauge theory description as $\mathrm{SU}(3)_{0}+10 \boldsymbol{F}$. The (flavor-equivalence classes of) box graphs encode the SU(3) gauge theory description of the descendant theory - this is written at the top of each box in the above graph. Knowing that the marginal theory enhances in the UV to the $6 \mathrm{~d}$ theory of $(\mathrm{SO}(10), \mathrm{SO}(10))$ minimal conformal matter, which is described by an affine $\mathrm{SO}(20)$ fiber, one can determine which of the curves corresponding to the weights of the $(\mathbf{3}, \boldsymbol{N})$ representation (where $N$ is the number of flavors in that flavor-equivalence class), and which of the $F_{i}$ associated to the roots of the affine $\mathrm{SO}(20)$ are contained inside of the non-flat surfaces. The intersection pattern of these curves is depicted via a dual graph in the lower half of each box. The superconformal flavor symmetry, $G_{\mathrm{F}}$ is obtained from the reduced CFDs, shown below the box graphs. 


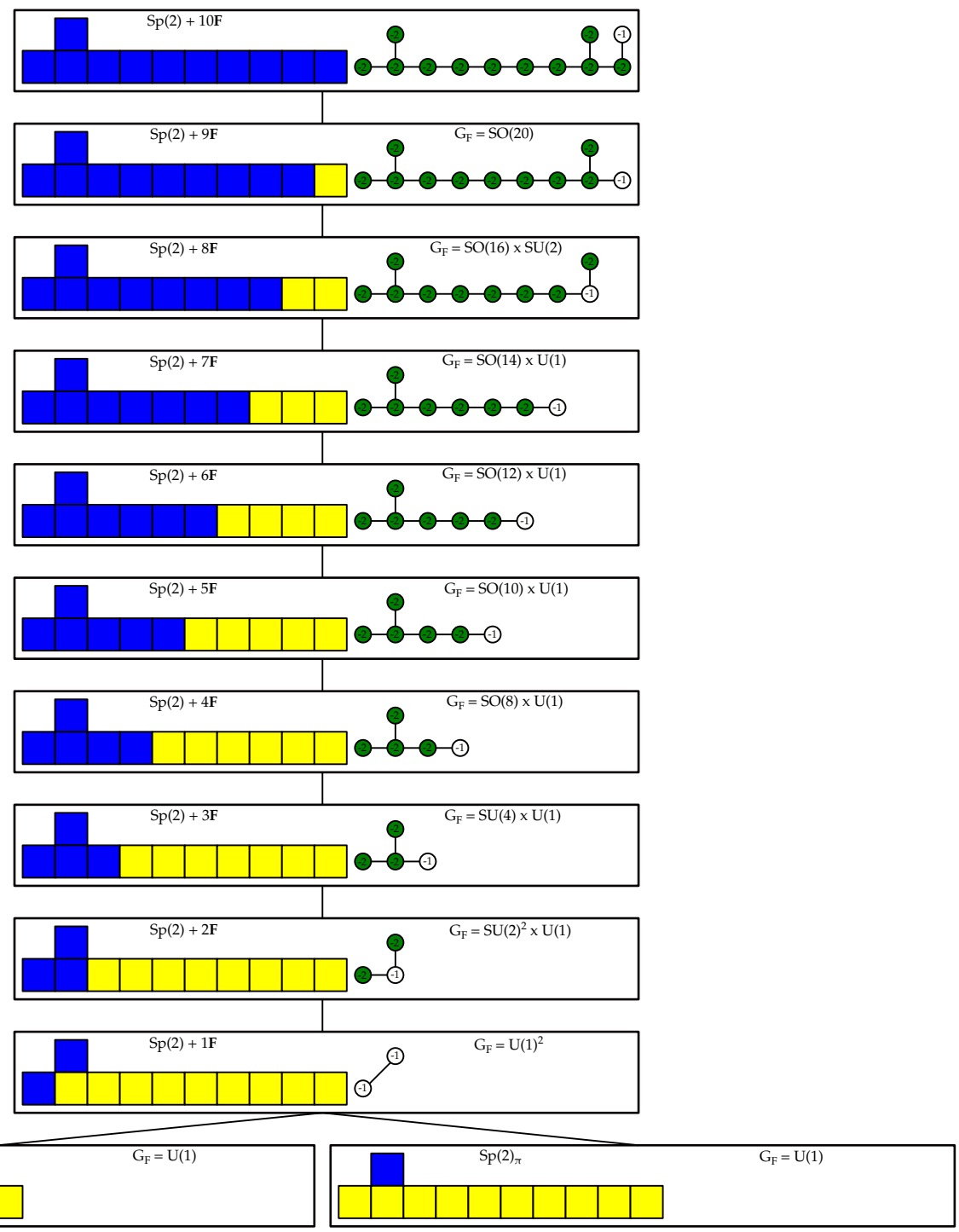

Figure 34. $\left(D_{5}, D_{5}\right)$ Conformal Matter: marginal theory description as $\operatorname{Sp}(2)+10 \boldsymbol{F}$. The figure shows the tree of descendant theories, together with their superconformal flavor symmetry, $G_{\mathrm{F}}$.

\section{A.3 SU(3) on a (-2)-curve with 6 hypermultiplets}

This theory has a marginal description in terms of

$$
\operatorname{Sp}(2)+3 \boldsymbol{A} \boldsymbol{S} .
$$

In this case we are considering the phase structure for a theory with gauge group

$$
\mathrm{Sp}(2)_{\text {gauge }} \times \mathrm{Sp}(3)_{\mathrm{BG}},
$$

with matter transforming in the $(\mathbf{5 , 6})$ representation. The highest weight of the $(\mathbf{5}, \mathbf{6})$ can be written as

$$
L_{1,1}=(0,1 ; 1,0,0) \text {. }
$$




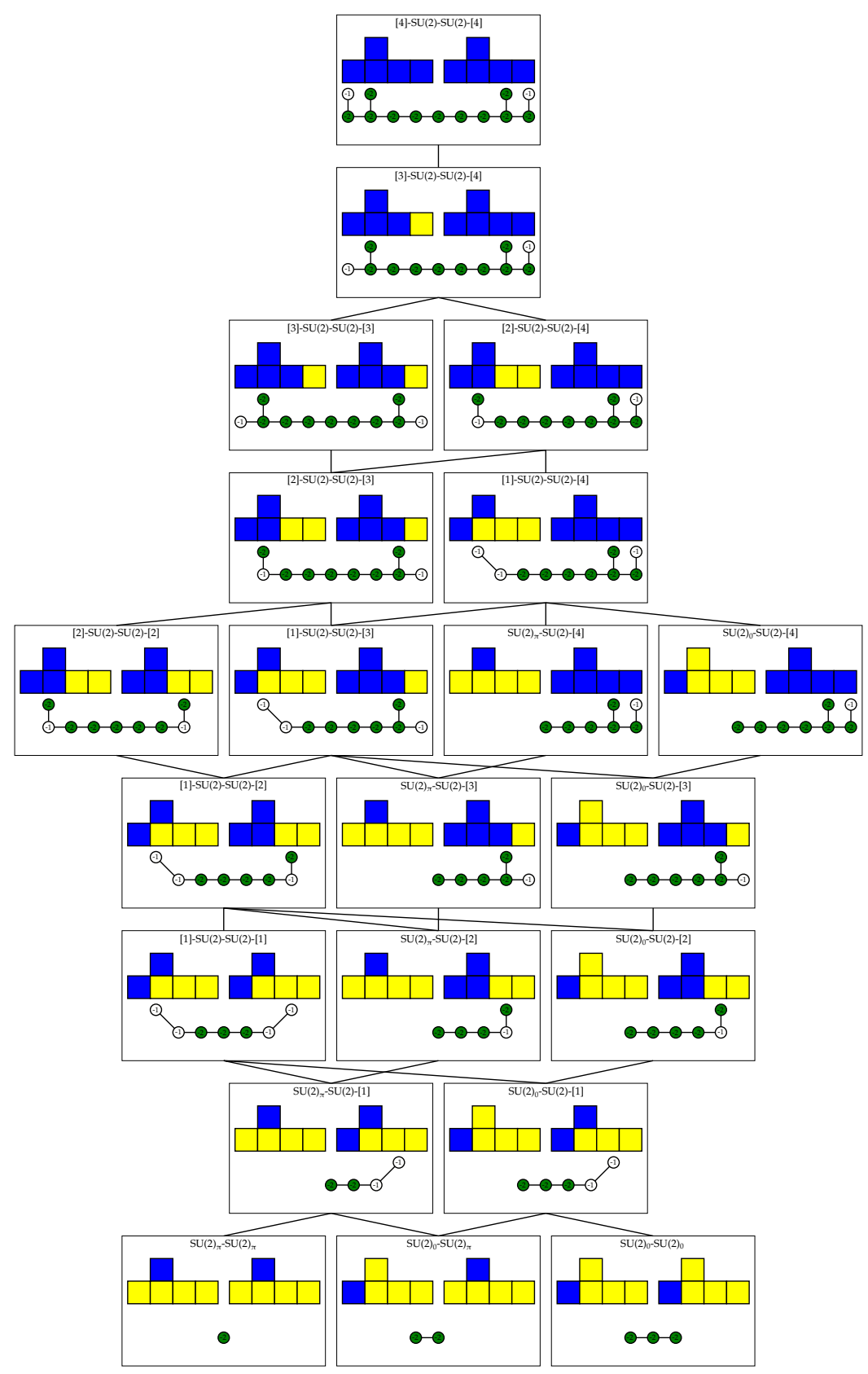

Figure 35. $\left(D_{5}, D_{5}\right)$ Conformal Matter: the marginal theory in the description as the quiver $4 \boldsymbol{F}-\mathrm{SU}(2)-\mathrm{SU}(2)-4 \boldsymbol{F}$. All of the descendant $5 \mathrm{~d}$ theories of such a $5 \mathrm{~d}$ marginal theory are given in the tree above, as determined through the flavor-equivalence classes of box graphs. The codimension two fiber, which is a splitting of affine $\mathrm{SO}(20)$, can be reconstructed in each case, and we draw the curves inside the fiber that are also contained inside of the compact surfaces in the lower half of each box. The reduced CFDs are shown from, which we determine the superconformal flavor symmetry. 


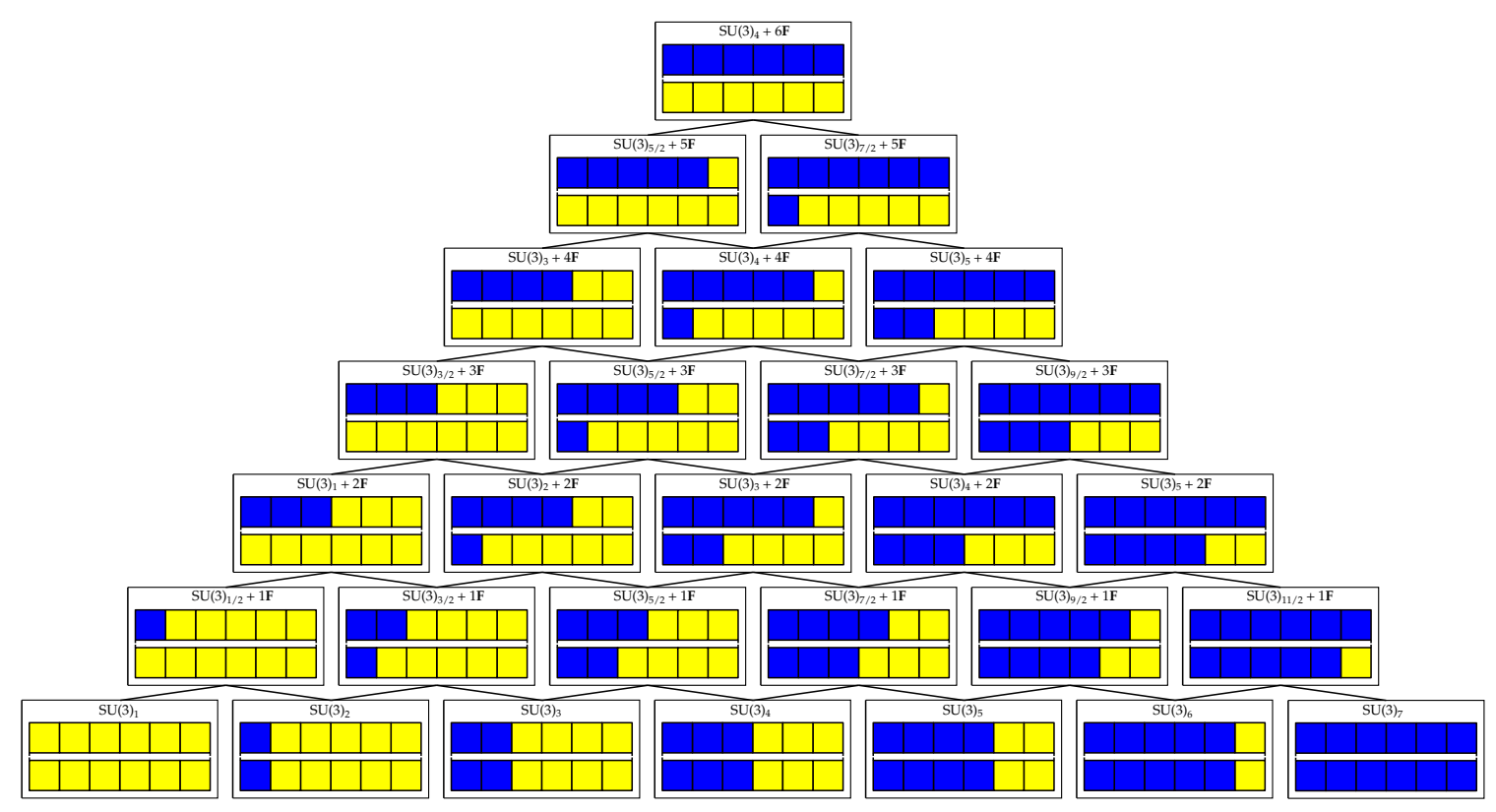

Figure 36. The tree of descendants for $\mathrm{SU}(3)_{4}+6 \boldsymbol{F}$, which is gauge theory description for the marginal theory obtained from $\mathrm{SU}(3)$ on a (-1)-curve with 12 hypermultiplets.

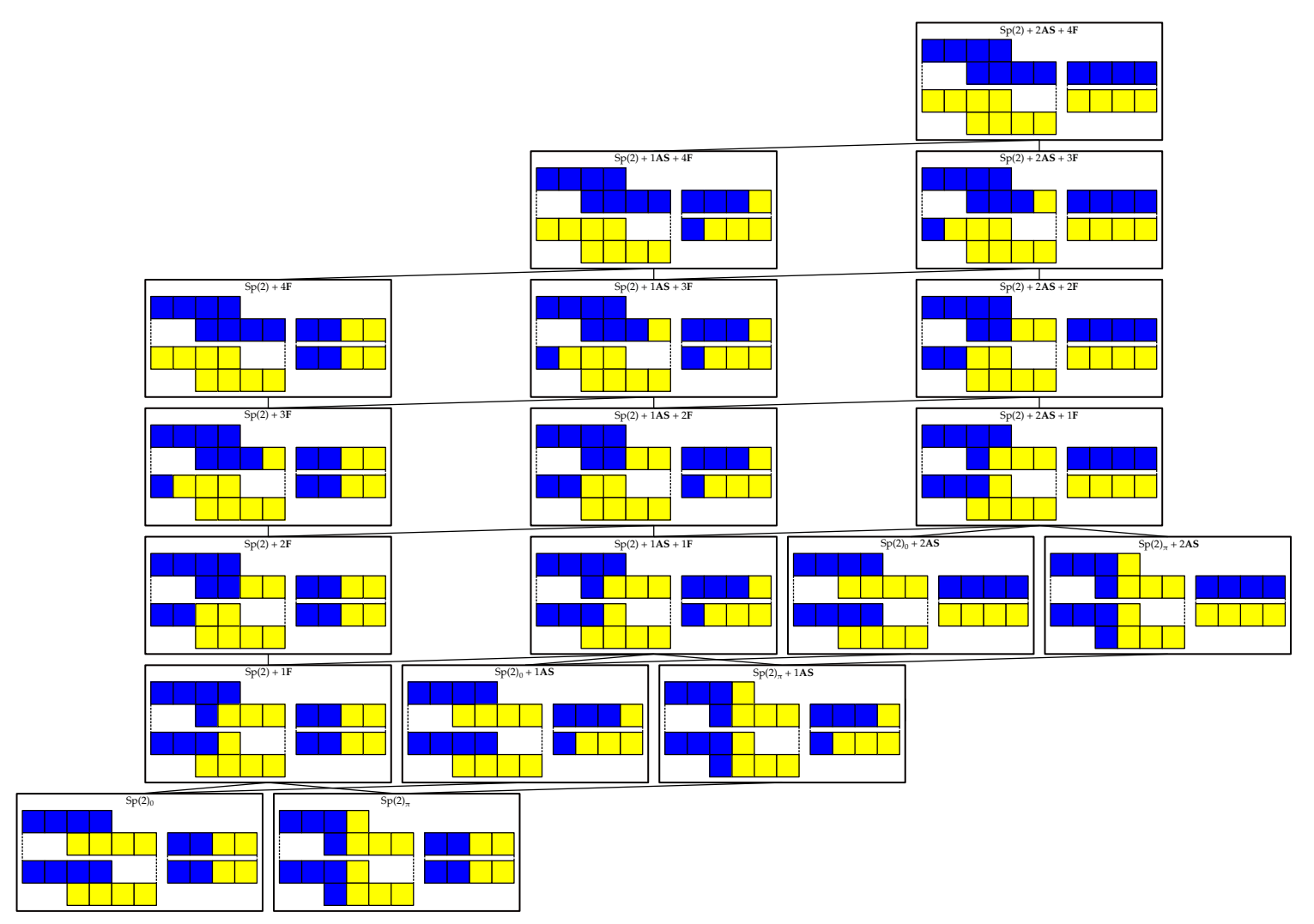

Figure 37. The tree of descendants for $\mathrm{Sp}(2)+6 \boldsymbol{F}+2 \boldsymbol{A S}$, which is gauge theory description for the marginal theory obtained from $\mathrm{SU}(3)$ on a $(-1)$-curve with 12 hypermultiplets. 


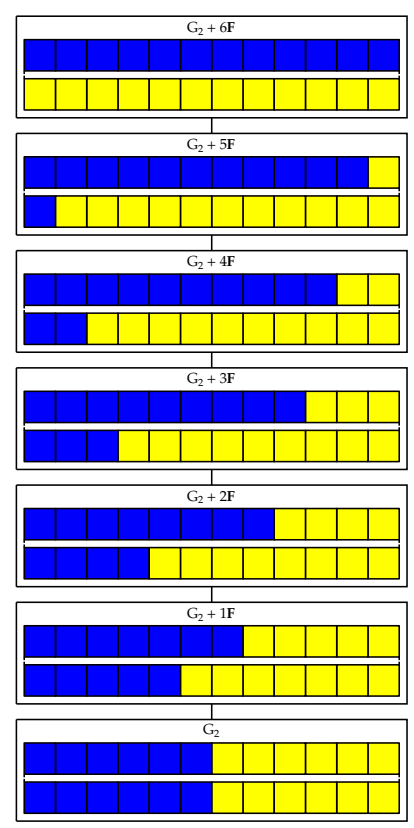

Figure 38. The tree of descendants for $G_{2}+6 \boldsymbol{F}$, which is gauge theory description for the marginal theory obtained from $\mathrm{SU}(3)$ on a $(-1)$-curve with 12 hypermultiplets.

The five simple roots of this semi-simple Lie algebra are, in the Cartan-Weyl basis,

$$
\begin{gathered}
\alpha_{1}^{2}=(2,-1 ; 0,0,0), \quad \alpha_{2}^{2}=(-2,2 ; 0,0,0) \\
\alpha_{1}^{3}=(0,0 ; 2,-1,0), \quad \alpha_{2}^{3}=(0,0 ;-1,2,-1), \quad \alpha_{3}^{3}=(0,0 ; 0,-2,2),
\end{gathered}
$$

where the superscript indicates which $\operatorname{Sp}(n)$ factor that it acts as the simple root of. The undecorated box graph, or the weight diagram, of this representation is depicted in figure 39. Furthermore, we can see directly from the self-conjugacy of the representation that determining all of the phases corresponds to determining the different consistent ways that signs can be assigned to the weights of the subgraph marked in red on figure 39 .

The consistent phases can be determined by the application of the flow rules to the decoration of the red-boxed subgraph. The total number of phases can be seen to be

$$
N_{\text {phases }}=10,
$$

and the adjacency graph can be determined in the usual manner. We are interested in the equivalence class of the phases where the same set of $\mathrm{Sp}(3)$ simple roots are contained inside of the splitting of the $\operatorname{Sp}(2)$ simple roots. Since the weight $-L_{3,3}$ is always associated to a minus sign we can see that this equivalence class is entirely specified by the signs of $L_{1,4}, L_{1,5}$, and $L_{1,6}$. The flop chain of these equivalence classes is drawn on the right in figure 39 .

\section{B Flavor symmetry enhancements for $\mathrm{SU}(N)_{k}+1 A S+N_{f} F$}

In this appendix we summary some of the known flavor symmetry enhacements of $5 \mathrm{~d}$ gauge theories of the type $\mathrm{SU}(N)_{k}+1 \boldsymbol{A} \boldsymbol{S}+N_{f} \boldsymbol{F}$ at their UV fixed points. These were determined 


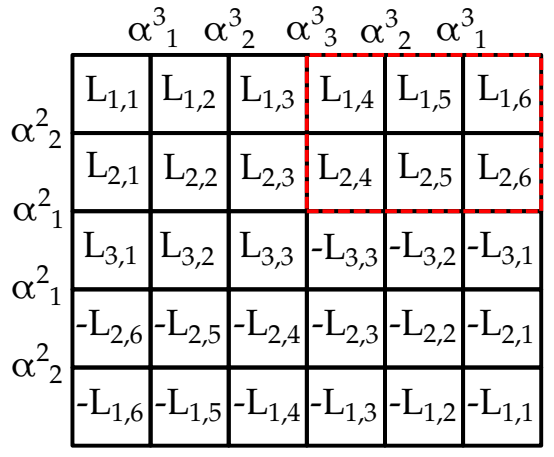

(a)

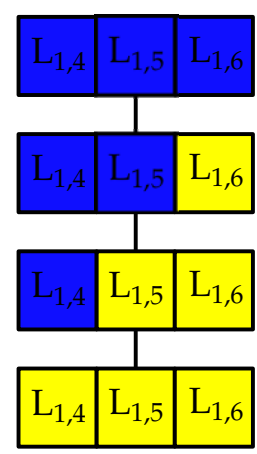

(b)

Figure 39. In (a) is the weight diagram of the $(\mathbf{5 , 6})$ representation of $\operatorname{Sp}(2) \times \operatorname{Sp}(3)$. We can see that, because the representation is self-conjugate, that the signs associated to $L_{i, j}$ for $i, j=1, \cdots, 3$ are required to be all plus. The subdiagram enclosed in red is then the only weights for whom the sign needs to be specified to fix the phase. The equivalence class of phases relevant in the limit where the gauge coupling of the $\operatorname{Sp}(3)$ is taking to zero is specified by the signs of the weights $L_{1,4}$, $L_{1,5}$, and $L_{1,6}$. In (b) is depicted the flop graph of these equivalence classes of phases.

from field theoretic methods, are contained implicitly in [33]. In particular, we focus on the cases, which descend from $\mathrm{SU}(2 n)_{0}+2 \boldsymbol{A} \boldsymbol{S}+8 \boldsymbol{F}$ and $\mathrm{SU}(2 n+1)_{0}+2 \boldsymbol{A} \boldsymbol{S}+8 \boldsymbol{F}$. We spell these out here, in order to facilitate the comparison with the CFDs. These flavor symmetry enhancements are summarized in tables $7,8,9,10,11$.

For $N$ even, i.e. $N=2 n$ and $n>2$ we always have that $N_{f}<N+4$, since $N_{f} \leq 8$. The superconformal flavor symmetry is related to the one of the theory where $1 \boldsymbol{A S}$ is decoupled in the following way

$$
G_{\mathrm{F}}=G_{\mathrm{F}}\left(\mathrm{SU}(2 n)_{k+n-2}+N_{f} \boldsymbol{F}\right) \times \mathrm{U}(1),
$$

where the extra $\mathrm{U}(1)$ is the classical symmetry acting on antisymmetric hypermultiplet, and $k$ shifts due to this decoupling, $k \rightarrow k^{\prime}=k+n-2$. The flavor symmetries for $\mathrm{SU}(2 n)_{k^{\prime}=k+n-2}+N_{f} \boldsymbol{F}$ can be obtained from $[1,38]$. In fact, in our cases, we have that

$$
\left|2 n-4-\frac{\left(8-N_{f}\right)}{2}\right| \leq\left|k^{\prime}\right| \leq 2 n-4+\frac{\left(8-N_{f}\right)}{2} .
$$

According to $[1,38]$, we have the following two distinct cases:

$$
\begin{aligned}
& 2 n-\frac{N_{f}}{2}>\left|k^{\prime}\right| \rightarrow G_{\mathrm{F}}\left(\mathrm{SU}(2 n)_{k^{\prime}=k+n-2}\right)=\mathrm{SU}\left(N_{f}\right) \times \mathrm{U}(1) \times \mathrm{U}(1) \\
& 2 n-\frac{N_{f}}{2}=\left|k^{\prime}\right|>\frac{1}{2} \quad \rightarrow \quad G_{\mathrm{F}}\left(\mathrm{SU}(2 n)_{k^{\prime}=k+n-2}\right)=\mathrm{SU}\left(N_{f}\right) \times \mathrm{SU}(2) \times \mathrm{U}(1) .
\end{aligned}
$$

For $N$ odd, i.e. $N=2 n+1$ and $n \geq 2, N_{f}<N+4$, since again we have that $N_{f} \leq 8$. The superconformal flavor symmetry is

$$
G_{\mathrm{F}}=G_{\mathrm{F}}\left(\mathrm{SU}(2 n+1)_{k+n-\frac{3}{2}}+N_{f} \boldsymbol{F}\right) \times \mathrm{U}(1),
$$


$k$ shifts, $k \rightarrow k^{\prime}=k+n-\frac{3}{2}$ when decoupling an antisymmetric hypermultiplet. The flavor symmetries for $\mathrm{SU}(2 n+1)_{k^{\prime}=k+n-\frac{3}{2}}+N_{f} \boldsymbol{F}$ can read from $[1,38]$. In fact, we have that

$$
\left|2 n-3-\frac{\left(8-N_{f}\right)}{2}\right| \leq\left|k^{\prime}\right| \leq 2 n-3+\frac{\left(8-N_{f}\right)}{2} .
$$

From $[1,38]$, we have

$$
\begin{aligned}
& 2 n+1-\frac{N_{f}}{2}>\left|k^{\prime}\right| \rightarrow G_{\mathrm{F}}\left(\mathrm{SU}(2 n)_{k^{\prime}=k+n-\frac{3}{2}}\right)=\mathrm{SU}\left(N_{f}\right) \times \mathrm{U}(1) \times \mathrm{U}(1) \\
& 2 n+1-\frac{N_{f}}{2}=\left|k^{\prime}\right|>\frac{1}{2} \quad \rightarrow \quad G_{\mathrm{F}}\left(\mathrm{SU}(2 n)_{k^{\prime}=k+n-\frac{3}{2}}\right)=\mathrm{SU}\left(N_{f}\right) \times \mathrm{SU}(2) \times \mathrm{U}(1) .
\end{aligned}
$$

\section{Details for geometric resolutions}

\section{C.1 Rank two E-string}

Here we present an explicit fiber geometry that resolves the non-minimal singularities of the $\left(E_{8}, \mathrm{SU}(2)\right)$ collision. It is obtained from a flop transition of the marginal geometry in figure 22 of [2]. Namely, the $(-1)$-curve $u_{8} \cdot S_{2}$ on $S_{2}$ is flopped into $S_{1}$. The resulting non-flat surfaces $S_{i}=\left\{\delta_{i}=0\right\}$ are shown in figure 40. Here, affine $E_{8}$ and $\mathrm{SU}(2)$ fiber components resolving the codimension one $E_{8}$ and $\mathrm{SU}(2)$ singularities, respectively, are

$$
\begin{aligned}
& \left(F_{0}^{E_{8}}, F_{1}^{E_{8}}, F_{2}^{E_{8}}, F_{3}^{E_{8}}, F_{4}^{E_{8}}, F_{5}^{E_{8}}, F_{6}^{E_{8}}, F_{7}^{E_{8}}, F_{8}^{E_{8}}\right) \\
\longleftrightarrow \quad & \left(U, u_{8}, u_{7}, u_{11}, u_{13}, u_{14}, u_{15}, u_{9}, u_{5}\right), \\
& \left(F_{0}^{\mathrm{SU}(2)}, F_{1}^{\mathrm{SU}(2)}\right) \longleftrightarrow\left(V, v_{1}\right) .
\end{aligned}
$$

As one can see from figure 40, the codimension one nodes $u_{7}$ and $v_{1}$ split into two components, each contained in one of the two surfaces $S_{1,2}$. The intersection numbers can be inferred from the homology classes, which we choose to represent in the basis of del Pezzo surfaces, i.e., $h^{2}=1, h \cdot e_{i}=0, e_{i} \cdot e_{j}=-\delta_{i j}$. In order to determine the gauge phases, it is important to remember that displayed curves are rational, and so we can compute for a curve $C \subset S_{i}$ the following intersection numbers in the three-fold:

$$
C \cdot S_{i}=-2-\left.(C \cdot C)\right|_{S_{i}}, \quad C \cdot S_{j}=\left.\left(C \cdot \delta_{j}\right)\right|_{S_{i}}, \quad \text { where } i \neq j .
$$

Note that the surfaces $S_{1}$ and $S_{2}$ contain the $(-1)$ curves labeled $e_{2}$ and $h-e_{1}-$ $e_{7}$, respectively, which do not arise from intersections with exceptional codimension one divisors. Together with the split products of $F_{1}^{\mathrm{SU}(2)} \leftrightarrow v_{1}$ and $F_{2}^{E_{8}} \leftrightarrow u_{7}$, and the gluing curve $S_{1} \cap S_{2}$, they form the extremal curves in this phase. For convenience, we list their 


\begin{tabular}{|c|c|c|c|c|c|}
\hline $\begin{array}{l}\underset{+}{+} \\
\stackrel{2}{2}\end{array}$ & & & & & 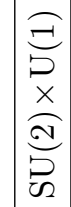 \\
\hline$\underset{\stackrel{+}{\rightleftarrows}}{\overrightarrow{+}}$ & & & & 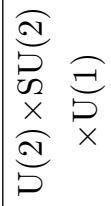 & 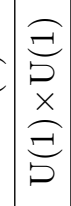 \\
\hline$\approx$ & & & 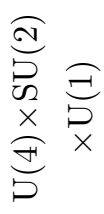 & 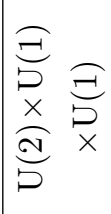 & 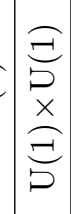 \\
\hline $\begin{array}{l}-7 \\
1 \\
\approx\end{array}$ & & 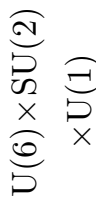 & 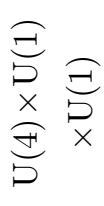 & 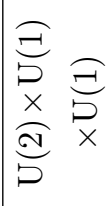 & 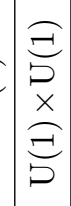 \\
\hline $\begin{array}{l}\mathcal{1} \\
1 \\
\approx\end{array}$ & 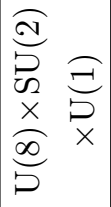 & 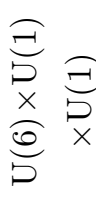 & 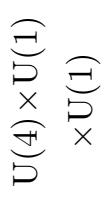 & 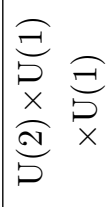 & 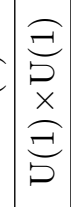 \\
\hline $\begin{array}{l}q \\
i \\
\varepsilon\end{array}$ & & 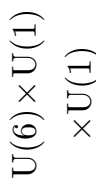 & 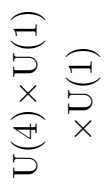 & 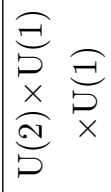 & 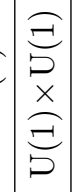 \\
\hline $\begin{array}{l}F \\
\dot{1} \\
\underline{E}\end{array}$ & & & 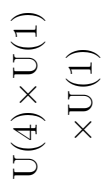 & 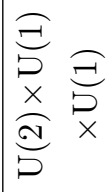 & 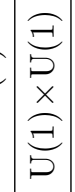 \\
\hline $\begin{array}{l}\overline{10} \\
1 \\
\varepsilon\end{array}$ & & & & 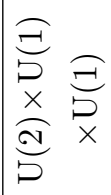 & 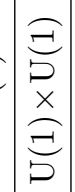 \\
\hline $\begin{array}{l}0 \\
1 \\
\Xi\end{array}$ & & & & & 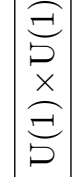 \\
\hline & $\infty$ & 0 & + & $\sim$ & 0 \\
\hline
\end{tabular}

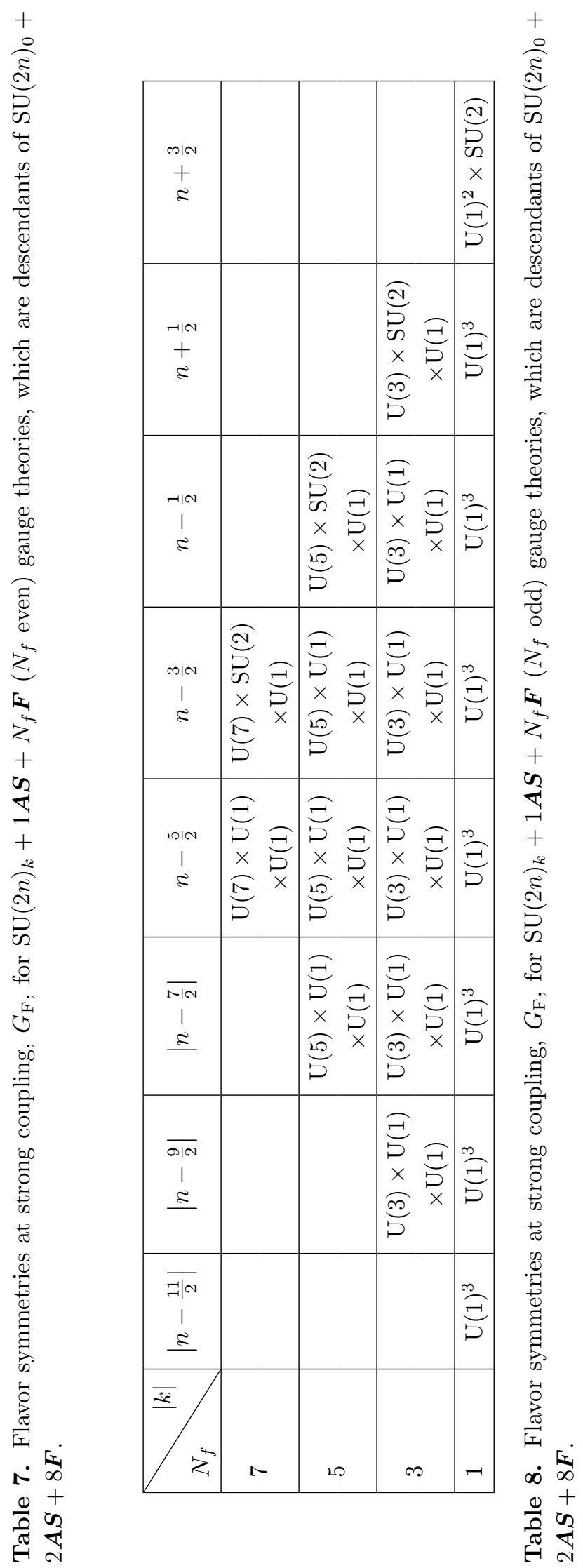




\begin{tabular}{|c|c|c|c|c|c|}
\hline 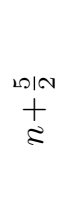 & & & & & 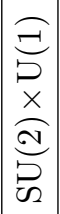 \\
\hline $\begin{array}{c}\text { min } \\
\stackrel{+}{\rightleftarrows}\end{array}$ & & & & 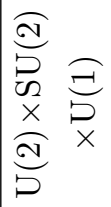 & 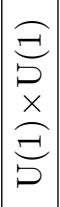 \\
\hline $\begin{array}{c}-1 \mathrm{~N} \\
\stackrel{+}{\approx}\end{array}$ & & & 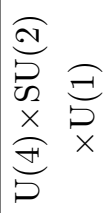 & 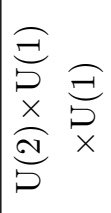 & 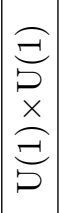 \\
\hline $\begin{array}{l}-\mid N \\
1 \\
\stackrel{N}{e}\end{array}$ & & 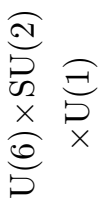 & 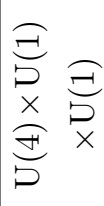 & 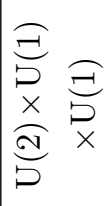 & 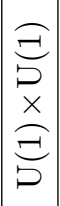 \\
\hline $\begin{array}{c}m / N \\
1 \\
\varepsilon\end{array}$ & 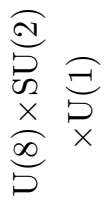 & 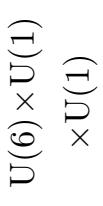 & 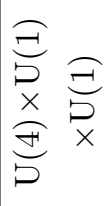 & 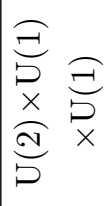 & 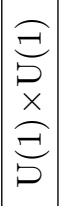 \\
\hline $\begin{array}{l}\mathrm{DIN} \\
1 \\
\varepsilon \\
\varepsilon\end{array}$ & & 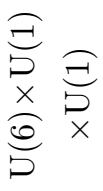 & 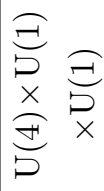 & 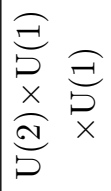 & 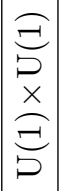 \\
\hline $\begin{array}{c}\overline{N-N} \\
1 \\
\varepsilon \\
\end{array}$ & & & 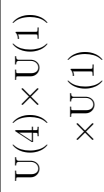 & 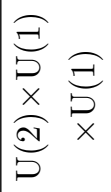 & 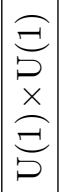 \\
\hline $\begin{array}{c}\overline{\sigma / N} \\
1 \\
\varepsilon \\
\end{array}$ & & & & 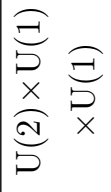 & 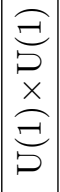 \\
\hline $\begin{array}{l}\exists \mid N \\
1 \\
\varepsilon\end{array}$ & & & & & 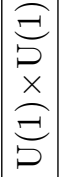 \\
\hline$z$ & $\infty$ & 0 & $r$ & N & 0 \\
\hline
\end{tabular}

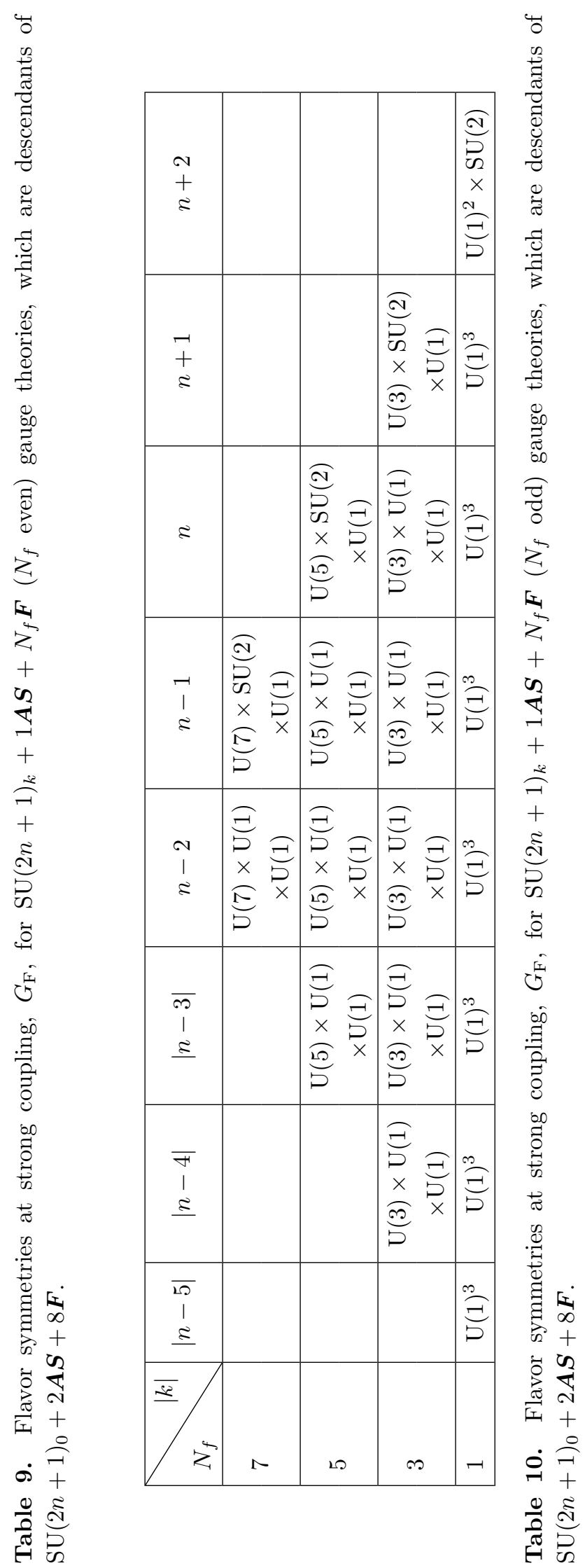




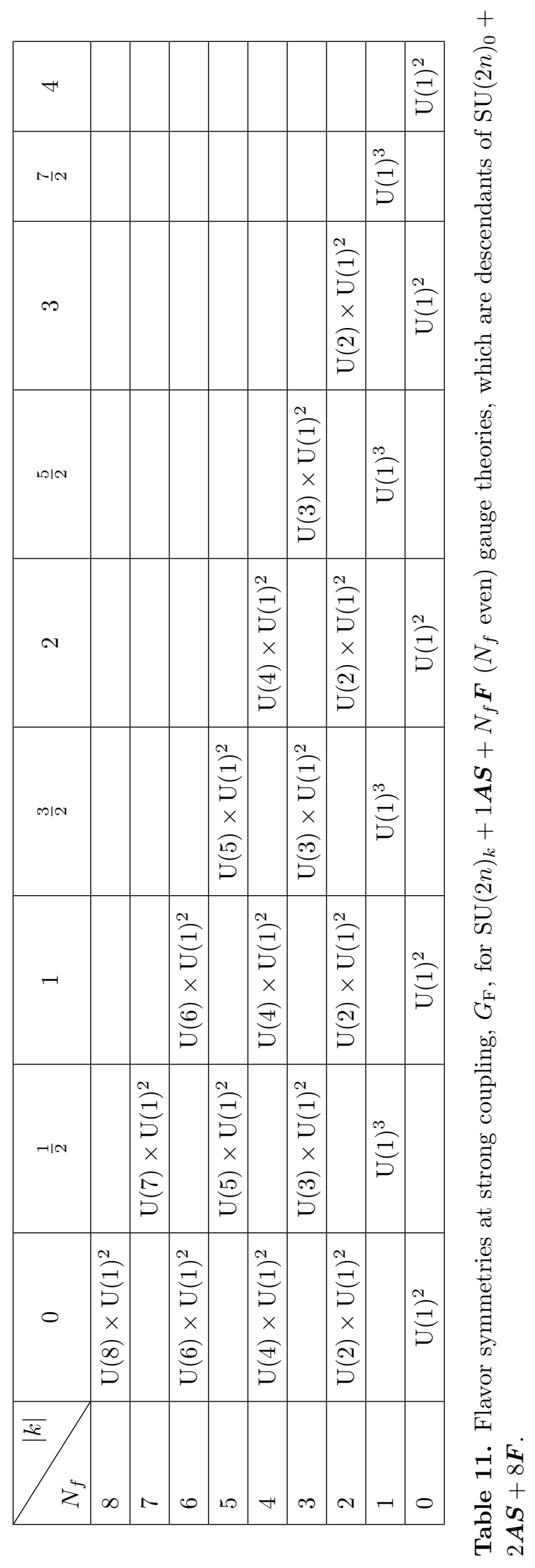




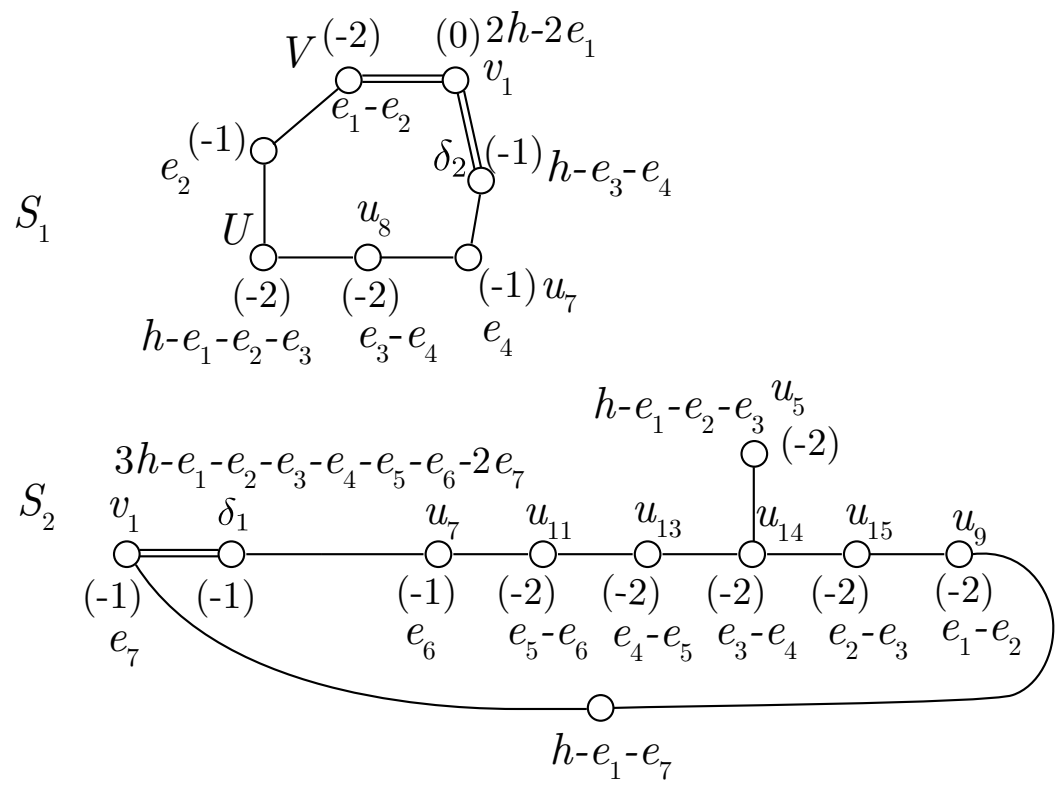

Figure 40. One concrete resolution of the $\left(E_{8}, \mathrm{SU}(2)\right)$ model that corresponds to a marginal theory in $5 \mathrm{~d}$. This geometry is obtained by a sequence of flops from the tensor branch resolution of the $6 \mathrm{~d}$ rank two E-string. One of these flops change the genus of the gluing curve $S_{1} \cap S_{2}=\left\{\delta_{1}\right\} \cap\left\{\delta_{2}\right\}$ from 1 to 0 . Each depicted node is rational curve generating the Mori cone of each the corresponding surface.

intersection numbers with all divisors:

\begin{tabular}{c|cc|cc|ccccccccc|cc} 
& $S_{1}$ & $S_{2}$ & \multicolumn{1}{|c|}{$D_{0}^{E_{8}}$} & $D_{1}^{E_{8}}$ & $D_{2}^{E_{8}}$ & $D_{3}^{E_{8}}$ & $D_{4}^{E_{8}}$ & $D_{5}^{E_{8}}$ & $D_{6}^{E_{8}}$ & $D_{7}^{E_{8}}$ & $D_{8}^{E_{8}}$ & $D_{0}^{\mathrm{SU}(2)} D_{1}^{\mathrm{SU}(2)}$ \\
\hline$\left.e_{2}\right|_{S_{1}}$ & -1 & 0 & 1 & 0 & 0 & 0 & 0 & 0 & 0 & 0 & 0 & 1 & 0 \\
\hline$\left.\left(h-e_{1}-e_{7}\right)\right|_{S_{2}}$ & 0 & -1 & 0 & 0 & 0 & 0 & 0 & 0 & 0 & 1 & 0 & 0 & 1 \\
\hline$\left.v_{1}\right|_{S_{2}}$ & 2 & -1 & 0 & 0 & 0 & 0 & 0 & 0 & 0 & 0 & 0 & 0 & -1 \\
\hline$\left.u_{7}\right|_{S_{1}}$ & -1 & 1 & 0 & 1 & -1 & 0 & 0 & 0 & 0 & 0 & 0 & 0 & 0 \\
\hline$\left.u_{7}\right|_{S_{2}}$ & 1 & -1 & 0 & 0 & -1 & 1 & 0 & 0 & 0 & 0 & 0 & 0 & 0 \\
\hline$S_{1} \cap S_{2}$ & -1 & -1 & 0 & 0 & 1 & 0 & 0 & 0 & 0 & 0 & 0 & 0 & 2
\end{tabular}

SU(3) gauge description. The rulings that give the $\mathrm{SU}(3)_{\text {gauge }}$ gauge theory are

$$
\begin{aligned}
& S_{1} \hookleftarrow f_{1}=\left.\left(h-e_{1}\right)\right|_{S_{1}}=\left.\left(e_{2}+U+u_{8}+u_{7}\right)\right|_{S_{1}}, \\
& S_{2} \hookleftarrow f_{2}=\left.\left(h-e_{7}\right)\right|_{S_{2}}=\left.\left(u_{7}+u_{11}+u_{13}+u_{14}+u_{15}+u_{9}+\left(h-e_{1}-e_{7}\right)\right)\right|_{S_{2}} .
\end{aligned}
$$

The codimension one fibers that are part of these rulings are the $E_{8} \operatorname{roots}\left(F_{0}^{E_{8}}, \cdots, F_{7}^{E_{8}}\right)$, which give rise to the non-abelian $\mathrm{SU}(9)_{\mathrm{BG}}$ part of the flavor symmetry $\mathrm{U}(9)_{\mathrm{BG}}$. We order the Cartan generators of $\mathrm{SU}(3)_{\text {gauge }}$ as $\left(S_{2}, S_{1}\right)$, and those of $\mathrm{SU}(9)_{\mathrm{BG}}$ as $\left(F_{1}, \cdots, F_{8}\right) \leftrightarrow$ $\left(F_{0}^{E_{8}}, \cdots, F_{7}^{E_{8}}\right)$, such that the geometry realizes hypermultiplets in the $(\mathbf{3}, \mathbf{9})$ representation, and not $(\overline{\mathbf{3}}, \mathbf{9})$. Furthermore, the extremal curves $\left.e_{2}\right|_{S_{1}},\left.u_{7}\right|_{S_{i}}$, and $\left.\left(h-e_{1}-e_{7}\right)\right|_{S_{2}}$ are special fiber components which shrink when we collapse $f_{1,2}$, and give rise to the bifundamental matter. 
$\operatorname{Sp}(2)$ gauge description. We obtain an $\operatorname{Sp}(2)_{\text {gauge }}$ gauge theory with the rulings

$$
\begin{aligned}
& S_{1} \hookleftarrow f_{1}=\left.\left(h-e_{1}\right)\right|_{S_{1}}=\left.\left(e_{2}+U+u_{8}+u_{7}\right)\right|_{S_{1}}, \\
& S_{2} \hookleftarrow f_{2}=\left.\left(v_{1}+\left(h-e_{1}-e_{7}\right)\right)\right|_{S_{2}}=\left.\left(u_{5}+u_{15}+2\left(u_{14}+u_{13}+u_{11}+u_{7}\right)\right)\right|_{S_{2}} .
\end{aligned}
$$

Here, the codimension one fibers $\left(F_{0}^{E_{8}}, \cdots, F_{6}^{E_{8}}, F_{8}^{E_{8}}\right)$ and $F_{1}^{\mathrm{SU}(2)}$ are parts of the ruling, giving rise to the flavor symmetry $\mathrm{SO}(16)_{\mathrm{BG}} \times \mathrm{SU}(2)_{\mathrm{BG}}$. Under the total symmetry group $\mathrm{Sp}(2)_{\text {gauge }} \times \mathrm{SO}(16)_{\mathrm{BG}} \times \mathrm{SU}(2)_{\mathrm{BG}}$, the extremal curves $\left.e_{2}\right|_{S_{1}}$ and $\left.u_{7}\right|_{S_{i}}$ give rise to states in the $(\mathbf{4}, \mathbf{1 6}, \mathbf{1})$, while $\left.v_{1}\right|_{S_{2}}$ and $\left.\left(h-e_{1}-e_{7}\right)\right|_{S_{2}}$ support $(\mathbf{5}, \mathbf{1}, \mathbf{2})$ states.

$\mathrm{SU}(2)^{2}$ gauge description. The rulings for this gauge theory are

$$
\begin{aligned}
& S_{1} \hookleftarrow f_{1}=\left.\left(\delta_{2}+u_{7}\right)\right|_{S_{1}}=\left.\left(U+2 e_{2}+V\right)\right|_{S_{1}}, \\
& S_{2} \hookleftarrow f_{2}=\left.\left(\delta_{1}+u_{7}\right)\right|_{S_{2}}=\left.\left(u_{5}+u_{13}+2\left(u_{14}+u_{15}+u_{9}+\left(h-e_{1}-e_{7}\right)\right)\right)\right|_{S_{2}} .
\end{aligned}
$$

The codimension one fibers contained in these rulings are $\left(F_{0}^{E_{8}}, F_{2}^{E_{8}}, F_{4}^{E_{8}}, F_{5}^{E_{8}}, \cdots, F_{8}^{E_{8}}\right)$ and $F_{0}^{\mathrm{SU}(2)}$, which span the flavor symmetry $(\mathrm{SO}(4) \times \mathrm{SU}(2) \times \mathrm{SO}(10))_{\mathrm{BG}}$. The extremal curves that give rise to matter are:

\begin{tabular}{c|c|ccc} 
curves & $\mathrm{SU}(2)_{\text {gauge }_{1}} \times \mathrm{SU}(2)_{\text {gauge }_{2}}$ & $\mathrm{SO}(4)_{\mathrm{BG}} \cong\left(\mathrm{SU}(2)^{2}\right)_{\mathrm{BG}} \mathrm{SU}(2)_{\mathrm{BG}} \mathrm{SO}(10)_{\mathrm{BG}}$ \\
\hline$\left.\delta_{2}\right|_{S_{1}}=\left.\delta_{1}\right|_{S_{2}}$, & $(\mathbf{2}, \mathbf{2})$ & $(\mathbf{1 , 1})$ & $\mathbf{2}$ & $\mathbf{1}$ \\
$\left.u_{7}\right|_{S_{1}},\left.u_{7}\right|_{S_{2}}$ & & $(\mathbf{2}, \mathbf{2})$ & $\mathbf{1}$ & $\mathbf{1}$ \\
\hline$\left.e_{2}\right|_{S_{1}}$ & $(\mathbf{2}, \mathbf{1})$ & $(\mathbf{1}, \mathbf{1})$ & $\mathbf{1}$ & $\mathbf{1 0}$
\end{tabular}

\section{C.2 Resolutions with different rulings for $\left(E_{6}, E_{6}\right)$ conformal matter}

The singular Tate model of $\left(E_{6}, E_{6}\right)$ conformal matter is

$$
y^{2}+b_{1} U V x y+b_{3} U^{2} V^{2} y=x^{3}+b_{2} U^{2} V^{2}+b_{4} U^{3} V^{3}+b_{6} U^{5} V^{5} .
$$

Here we present two example resolutions, which have different ruling and quiver gauge theory descriptions. The first one is

$$
\begin{aligned}
& B U 1_{\left(E_{6}, E_{6}\right)}= \\
& \left\{\left\{x, y, U, u_{1}\right\},\left\{x, y, V, v_{1}\right\},\left\{x, y, u_{1}, u_{2}\right\},\left\{y, u_{1}, u_{2}, u_{3}\right\},\left\{y, u_{1}, u_{4}\right\},\left\{y, u_{2}, u_{5}\right\},\left\{v_{1}, u_{5}, \delta_{1}\right\},\right. \\
& \left\{\delta_{1}, y, \delta_{2}\right\},\left\{v_{1}, u_{4}, \delta_{3}\right\},\left\{v_{1}, \delta_{2}, \delta_{4}\right\},\left\{u_{3}, u_{4}, u_{6}\right\},\left\{y, u_{3}, u_{7}\right\},\left\{x, y, v_{1}, v_{2}\right\},\left\{y, v_{1}, v_{2}, v_{3}\right\}, \\
& \left.\left\{y, v_{1}, v_{4}\right\},\left\{y, v_{2}, v_{5}\right\},\left\{v_{3}, v_{4}, v_{6}\right\},\left\{y, v_{3}, v_{7}\right\},\left\{u_{7}, v_{1}, \delta_{5}\right\}\right\} .
\end{aligned}
$$

The following exceptional divisors form the Dynkin diagram of $\left(E_{6}, E_{6}\right)$ :

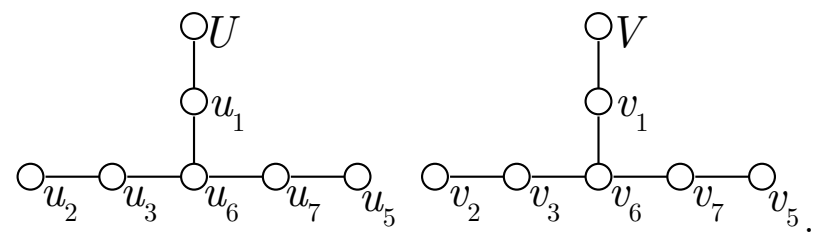


We list some of the triple intersection numbers here:

\begin{tabular}{c|ccccccccccccccccccc}
$S_{i} \cdot D_{j}^{2}$ & $U$ & $u_{1}$ & $u_{6}$ & $u_{3}$ & $u_{2}$ & $u_{7}$ & $u_{5}$ & $V$ & $v_{1}$ & $v_{6}$ & $v_{3}$ & $v_{2}$ & $v_{7}$ & $v_{5}$ & $\delta_{1}$ & $\delta_{2}$ & $\delta_{3}$ & $\delta_{4}$ & $\delta_{5}$ \\
\hline$S_{1}$ & 0 & 0 & 0 & 0 & -2 & 0 & 0 & 0 & 0 & 0 & 0 & 0 & 0 & 0 & 8 & 0 & 0 & 2 & 0 \\
$S_{2}$ & 0 & 0 & 0 & 0 & 0 & 0 & -2 & 0 & 0 & 0 & 0 & 0 & 0 & 0 & 0 & 8 & 0 & 2 & 0 \\
$S_{3}$ & 0 & -1 & -2 & -1 & 0 & -1 & 0 & 0 & 0 & 0 & 0 & 0 & 0 & 0 & 0 & 0 & 6 & 0 & -2 \\
$S_{4}$ & 0 & 0 & 0 & -1 & 0 & -1 & 0 & 0 & 0 & -2 & -2 & -2 & -2 & -2 & -4 & -4 & 0 & 0 & -2 \\
$S_{5}$ & 0 & 0 & 0 & 1 & 0 & 1 & 0 & 0 & -1 & 0 & 0 & 0 & 0 & 0 & 0 & 0 & 0 & 0 & 8 \\
\hline$n\left(F_{j}\right)$ & 0 & -1 & -2 & -2 & -2 & -2 & -2 & 0 & -2 & -2 & -2 & -2 & -2 & -2 & - & - & - & - & -
\end{tabular}

$n\left(F_{j}\right)$ is the "wrapping number" of each Cartan node inside the non-flat fiber, introduced in [2]. If $n\left(F_{j}\right)=-2$, then such node is fully wrapped and regarded as a flavor curve. In this case, the actual wrapping number $n\left(F_{j}\right)$ are computed with the following non-trivial multiplicities [2]:

$$
\xi_{i}^{(u)}=(1,1,1,1,2), \xi_{i}^{(v)}=(1,1,1,2,1) .
$$

As a reminder, any intersection numbers $S_{i} \cdot u_{j}^{2}$ needs to be multiplied by $\xi_{i}^{(v)}$, while any intersection numbers $S_{i} \cdot v_{j}^{2}$ needs to be multiplied by $\xi_{i}^{(u)}$ (including the affine nodes $U$ and $V)$.

We draw the configuration of curves on the five surface components in figure 41 . The corresponding CFD is read off as

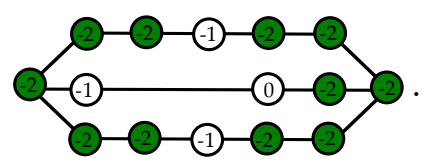

In this case, the assignment of ruling and section for each surface and each curve is uniquely determined. Recall that the ruling curve on each surface needs to be a linear combination of curves with self-intersection number 0 and genus 0 . Since $S_{1}, S_{2}$ and $S_{5}$ are Hirzebruch surfaces, the 0-curves on them have to be ruling curves. Then we can conclude that $S_{1} \cdot S_{4}, S_{2} \cdot S_{4}$ are section curves, while $S_{3} \cdot S_{5}$ and $S_{4} \cdot S_{5}$ are a part of ruling curves. If the geometry has a quiver gauge theory description, then the assignment of section and ruling needs to be identical for a curve $S_{i} \cdot S_{j}$ on both $S_{i}$ and $S_{j}$. With these requirements, the only consistent assignment of section/ruling is shown in figure 41 . We also list the linear combinations of curves on each surface component that correspond to the ruling:

$$
\begin{aligned}
& S_{1}: f^{(1)} \equiv x=u_{3}, \\
& S_{2}: f^{(2)} \equiv x=u_{7}, \\
& S_{3}: f^{(3)} \equiv V=\delta_{5}+2 u_{3}+u_{6}, \\
& S_{4}: f^{(4)} \equiv u_{3}+\delta_{5}+u_{7}=C_{1}+v_{2}+v_{3}+v_{6}+v_{7}+v_{5}+C_{2}, \\
& S_{5}: f^{(5)} \equiv \delta_{4}=\delta_{3} .
\end{aligned}
$$

From this information, we see that the quiver gauge theory has a $\mathrm{SU}(4) \times \mathrm{SU}(2)^{(1)} \times$ $\mathrm{SU}(2)^{(2)}$ gauge group. The three Cartans generators of SU(4) correspond to $S_{1}, S_{4}$ and $S_{2}$, while the Cartan generators of $\mathrm{SU}(2)^{(1)}$ and $\mathrm{SU}(2)^{(2)}$ correspond to $S_{5}$ and $S_{3}$ respectively. 


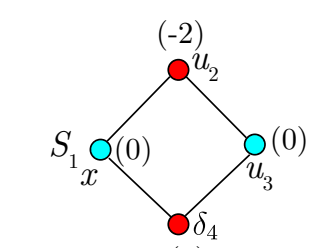

(2)

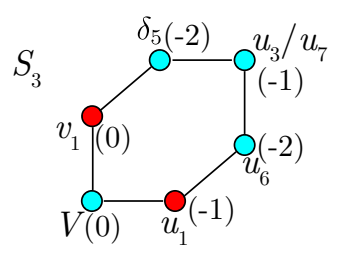

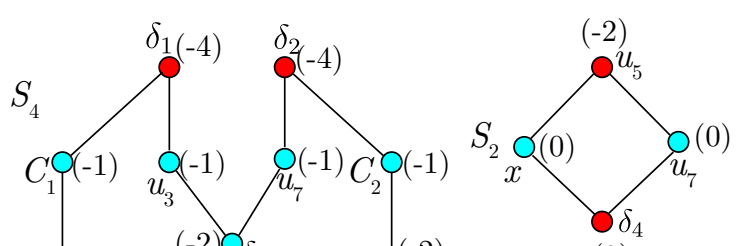

$(2)$

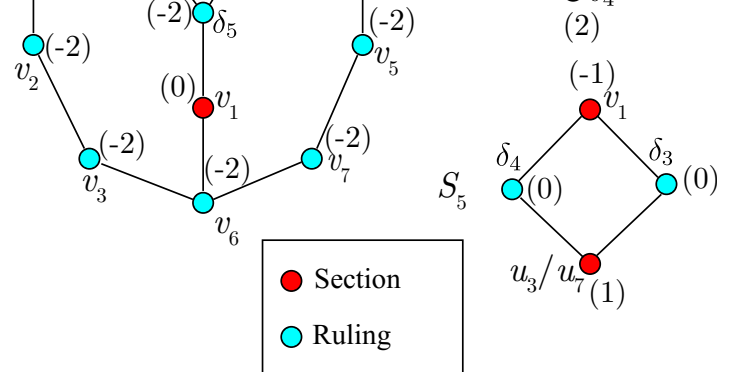

Figure 41. The configuration of curves on $S_{i}(i=1, \ldots, 5)$ in the geometry $B U 1_{\left(E_{6}, E_{6}\right)}$. The number in the bracket denotes the self-intersection number of the curve. The letter denotes an intersection curve with the corresponding divisor. The "/" symbol means that the curves are in the same homology class. The assignment of section/ruling of each curve is marked by red/blue colors.

The massless matter fields are generated by M2 brane wrapping over ( -1$)$-curves that are apart of the rulings, which shrinks to zero size in the gauge theory limit. The two unlabeled (-1)-curves on $S_{2}$, along with their linear combinations with the string of five $(-2)$-curves, in total give six copies of $(\mathbf{4}, \mathbf{1}, \mathbf{1})$ and $(\overline{\mathbf{4}}, \mathbf{1}, \mathbf{1})$ under $\mathrm{SU}(4) \times \mathrm{SU}(2)^{(1)} \times$ $\mathrm{SU}(2)^{(2)}$. Additionaly, the curves $u_{3} \cdot S_{2}$ and $u_{7} \cdot S_{2}$ give rise to bifundamentals $(\overline{\mathbf{4}}, \mathbf{1}, \mathbf{2})$ and $(\mathbf{4}, \mathbf{1}, \mathbf{2})$. Moreover, the curve $u_{3} \cdot S_{3}$ gives the bifundamental $(\mathbf{1}, \mathbf{2}, \mathbf{2})$.

In conclusion, the quiver gauge theory description of the geometry $B U 1_{\left(E_{6}, E_{6}\right)}$ is

$$
[6]-\mathrm{SU}(4)-\mathrm{SU}(2)^{(1)}-\mathrm{SU}(2)^{(2)} .
$$

From the geometry in figure 41, we can blow up the surface component $S_{3}$ twice and get the following configuration:

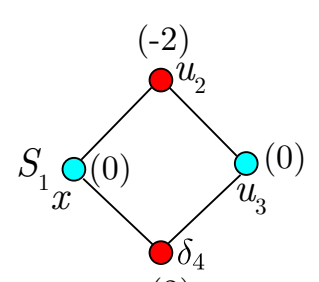

(2)

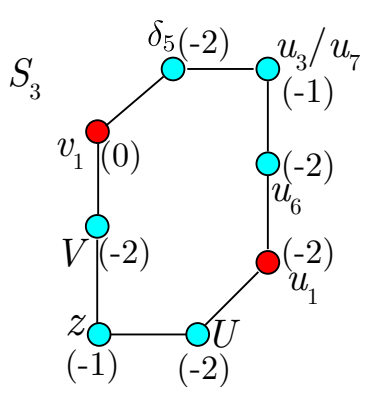

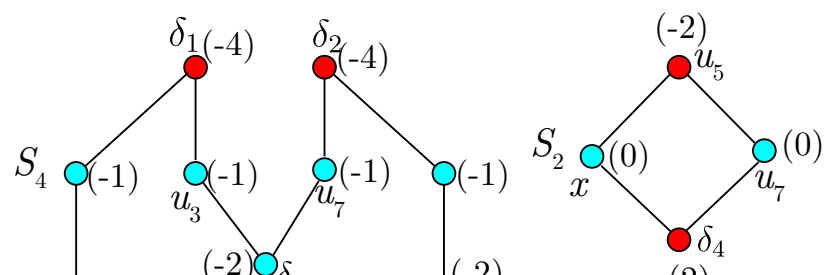

(2)

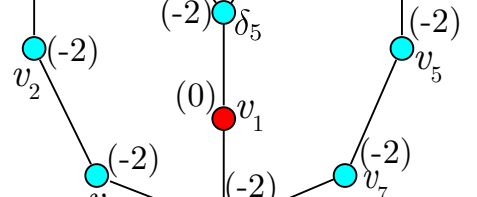

$S_{5}$

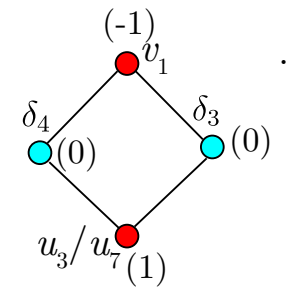


Because of the appearance of new fibral $(-1)$-curve $z \cdot S_{3}$ on $S_{3}$ and its two adjacent $(-2)$-curves, the quiver gauge theory description is now

$$
[6]-\mathrm{SU}(4)-\mathrm{SU}(2)^{(1)}-\mathrm{SU}(2)^{(2)}-[2],
$$

with two fundamental flavors on the $\mathrm{SU}(2)^{(2)}$ gauge node. Moreover, the corresponding CFD of (C.16) is exactly the marginal CFD of $\left(E_{6}, E_{6}\right)$ conformal matter theory:

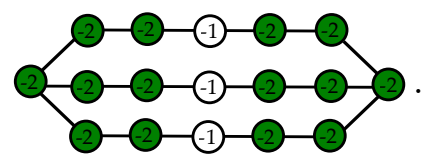

Hence we conclude that the geometry (C.16) describes the $\left(E_{6}, E_{6}\right)$ marginal theory, and the quiver description (6.1) in section 6.1.1 indeed appears.

From the geometry (C.16), there are two ways to flop a (-1)-curve outside of these surfaces. One can shrink the $(-1)$-curve $z \cdot S_{3}$ on $S_{3}$, and get the following geometry:

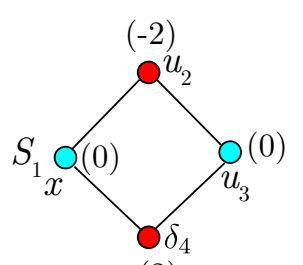

$(2)$

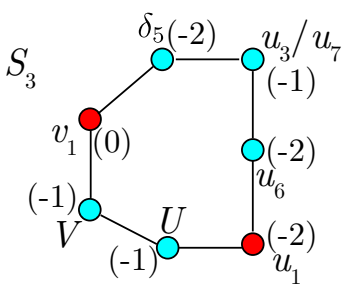

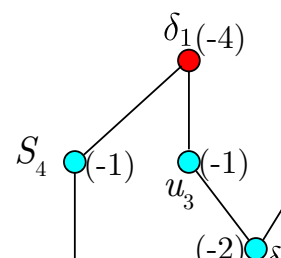

$\mathrm{O}(-2)$

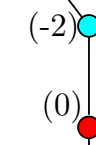

(0) $\delta^{2}(-4)$

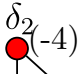

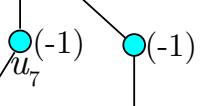<smiles>[SiH3]O[GeH2]O[GeH2]</smiles>

$(2)$

It has quiver gauge theory description

$$
[6]-\mathrm{SU}(4)-\mathrm{SU}(2)^{(1)}-\mathrm{SU}(2)^{(2)}-[1] \text {. }
$$

Alternatively, one can flop the (-1)-curve connected to $v_{2} \cdot S_{4}$ on $S_{4}$ into $S_{1}$ and then shrink it. After this birational transformation, the surface geometry becomes

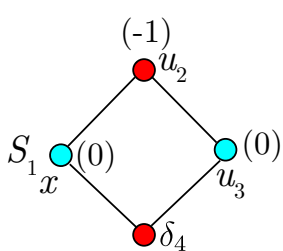

(1)

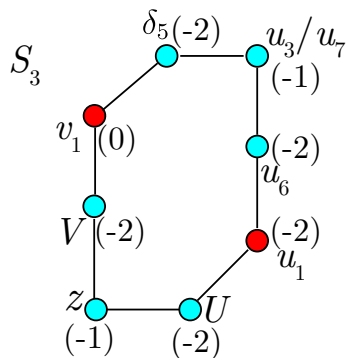

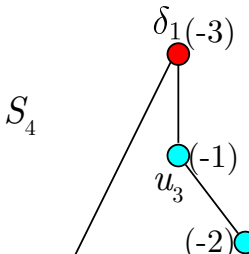

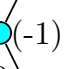

Section

O Ruling 
Comparing to (C.16), the number of fundamental flavors for the $\mathrm{SU}(4)$ gauge group is decreased by one, and we have the quiver gauge theory description

$$
[5]-\mathrm{SU}(4)-\mathrm{SU}(2)^{(1)}-\mathrm{SU}(2)^{(2)}-[2] \text {. }
$$

Notably, the two different geometries (C.19) and (C.21) have the same CFD:

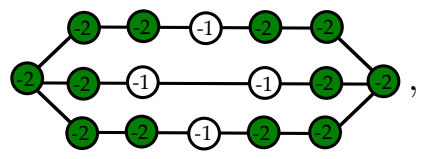

with superconformal flavor symmetry $G_{\mathrm{F}}=E_{6} \times E_{6}$.

This confirms the non-trivial UV duality between the two quiver gauge theory descriptions [6] - $\mathrm{SU}(4)-\mathrm{SU}(2)-\mathrm{SU}(2)-[1]$ and [5] $-\mathrm{SU}(4)-\mathrm{SU}(2)-\mathrm{SU}(2)-[2]$ in section 6.1.1, from the geometric perspective.

Furthermore, we can flop the (-1)-curve connected to $v_{2} \cdot S_{4}$ on $S_{4}$ into $S_{1}$ and then shrink it, resulting in the geometry

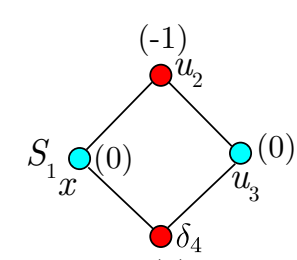

(1)

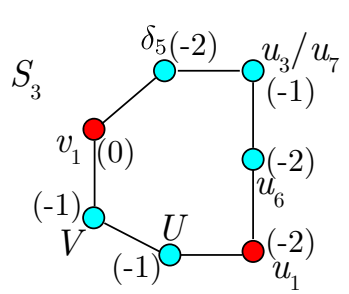

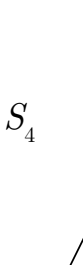

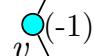

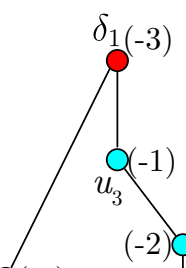
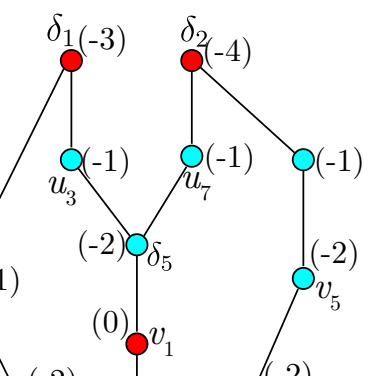

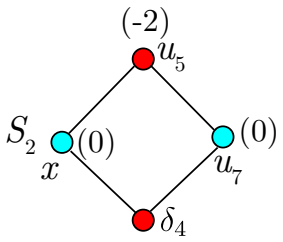

$(2)$

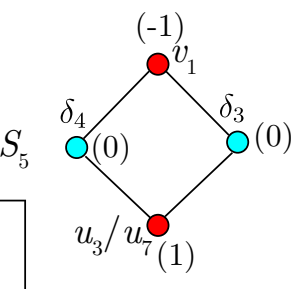

with the quiver gauge theory description

$$
[5]-\mathrm{SU}(4)-\mathrm{SU}(2)^{(1)}-\mathrm{SU}(2)^{(2)}-[1] \text {. }
$$

From (C.21), we can shrink the (-1)-curve $U \cdot S_{3}$ on $S_{3}$ to get the geometry (C.24). Alternatively, we can flop the (-1)-curve $v_{2} \cdot S_{4}$ on $S_{4}$ into $S_{1}$ and then shrink it, resulting in the geometry

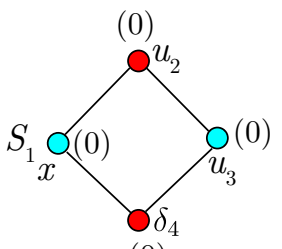

(0)

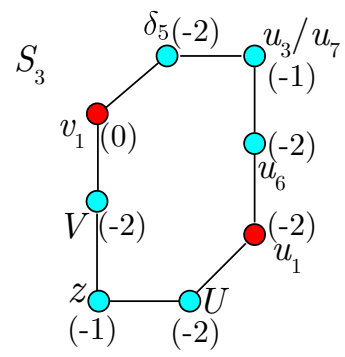

$(-2)$

$S_{4} \delta_{1}$

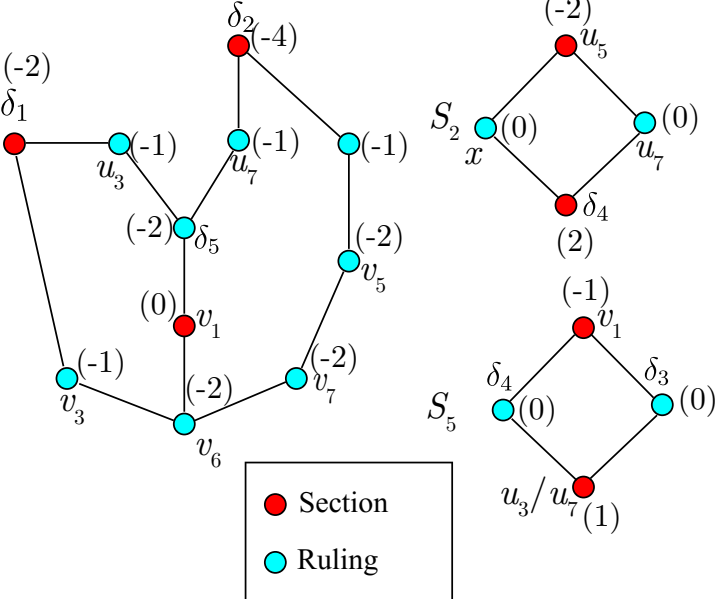


with the quiver gauge theory description

$$
[4]-\mathrm{SU}(4)-\mathrm{SU}(2)^{(1)}-\mathrm{SU}(2)^{(2)}-[2] .
$$

The geometries with quiver gauge theory descriptions [6] $-\mathrm{SU}(4)-\mathrm{SU}(2)-\mathrm{SU}(2)$ in figure 41 and [4] $-\mathrm{SU}(4)-\mathrm{SU}(2)-\mathrm{SU}(2)-[2]$ in (C.26) correspond to the same CFD:

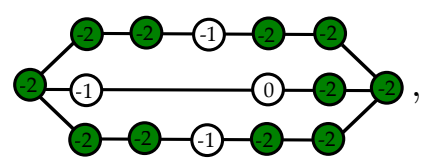

with the same $G_{\mathrm{F}}=E_{6} \times \mathrm{SU}(6)$. Hence we can perceive the UV duality between these two quiver gauge theories.

One the other hand, the geometry (C.24) with quiver gauge theory description [5] $\mathrm{SU}(4)-\mathrm{SU}(2)-\mathrm{SU}(2)-[1]$ corresponds to a different CFD:

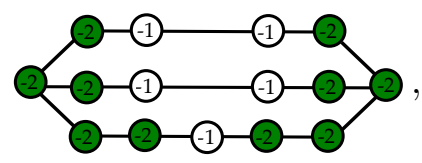

with $G_{\mathrm{F}}=\mathrm{SO}(10)^{2} \times \mathrm{U}(1)$.

Apart from this class of resolution geometries, we can also choose another resolution sequence:

$$
\begin{aligned}
& B U 2_{\left(E_{6}, E_{6}\right)}= \\
& \left\{\left\{x, y, V, v_{1}\right\},\left\{x, y, U, u_{1}\right\},\left\{x, y, u_{1}, u_{2}\right\},\left\{x, y, v_{1}, v_{2}\right\},\left\{y, u_{1}, u_{2}, u_{3}\right\},\left\{y, v_{1}, v_{2}, v_{3}\right\},\right. \\
& \left\{y, v_{1}, v_{4}\right\},\left\{u_{2}, v_{4}, \delta_{1}\right\},\left\{y, \delta_{1}, \delta_{2}\right\},\left\{\delta_{1}, v_{4}, \delta_{4}\right\},\left\{v_{4}, u_{3}, \delta_{5}\right\},\left\{v_{4}, u_{1}, \delta_{3}\right\}, \\
& \left.\left\{y, u_{1}, u_{4}\right\},\left\{y, u_{2}, u_{5}\right\},\left\{u_{3}, u_{4}, u_{6}\right\},\left\{y, u_{3}, u_{7}\right\},\left\{y, v_{2}, v_{5}\right\},\left\{v_{3}, v_{4}, v_{6}\right\},\left\{y, v_{3}, v_{7}\right\}\right\} .
\end{aligned}
$$

The multiplicities are the same as (C.12), and we have the following intersection numbers:

\begin{tabular}{c|ccccccccccccccccccc}
$S_{i} \cdot D_{j}^{2}$ & $U$ & $u_{1}$ & $u_{6}$ & $u_{3}$ & $u_{2}$ & $u_{7}$ & $u_{5}$ & $V$ & $v_{1}$ & $v_{6}$ & $v_{3}$ & $v_{2}$ & $v_{7}$ & $v_{5}$ & $\delta_{1}$ & $\delta_{2}$ & $\delta_{3}$ & $\delta_{4}$ & $\delta_{5}$ \\
\hline$S_{1}$ & 0 & 0 & 0 & 0 & 0 & 0 & 0 & 0 & 0 & 0 & 0 & 0 & 0 & 0 & 8 & 0 & 0 & 0 & 0 \\
$S_{2}$ & 0 & 0 & 0 & 0 & 0 & -1 & 0 & 0 & 0 & -1 & 0 & 0 & -1 & 0 & 0 & 6 & 0 & -2 & -1 \\
$S_{3}$ & 0 & -1 & 0 & 0 & 0 & 0 & 0 & 0 & 1 & 0 & 0 & 0 & 0 & 0 & 0 & 0 & 8 & 0 & 0 \\
$S_{4}$ & 0 & 0 & 0 & -1 & 0 & 0 & 0 & 0 & 0 & -1 & -1 & 0 & 0 & 0 & -2 & 0 & 0 & 6 & -1 \\
$S_{5}$ & 0 & -1 & -2 & 0 & 0 & -1 & 0 & 0 & -1 & 0 & 0 & 0 & 0 & 0 & 0 & -1 & -2 & -1 & 5 \\
\hline$n\left(F_{j}\right)$ & 0 & -2 & -2 & -2 & 0 & -2 & 0 & 0 & -1 & -2 & -1 & 0 & -1 & 0 & - & - & - & - & -
\end{tabular}

The corresponding CFD is

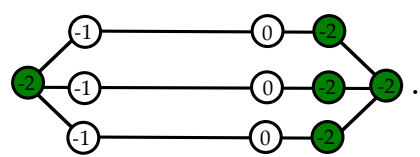

We plot the configuration of curves on the five non-flat surface components in figure 42. For this geometry, the assignment of ruling on each surface component is uniquely fixed by 

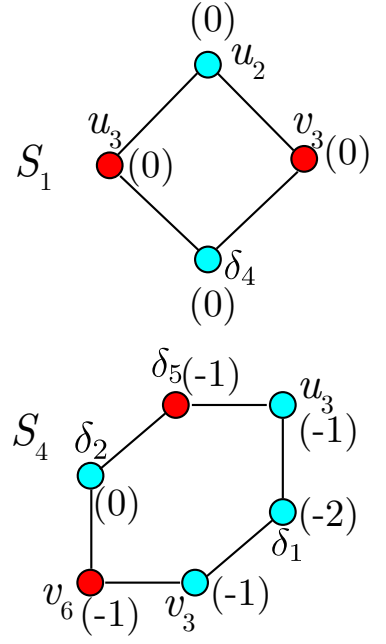
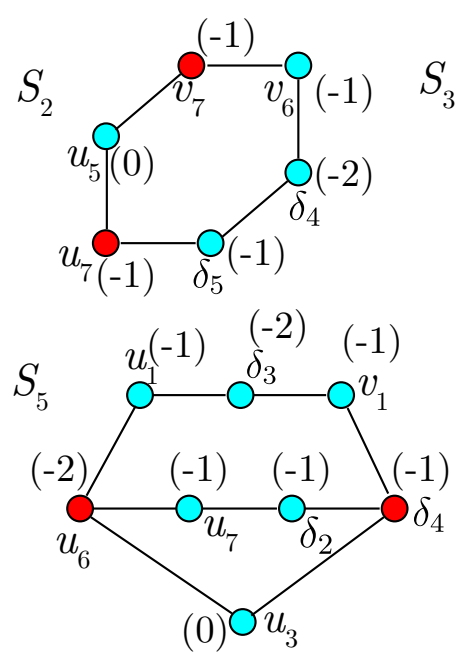

(1)

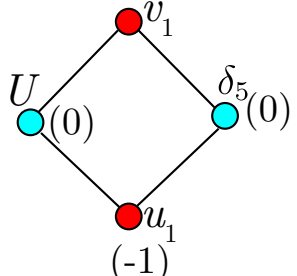

\section{Section \\ Ruling}

Figure 42. The configuration of curves on $S_{i}(i=1, \ldots, 5)$ in the resolution geometry $B U 2_{\left(E_{6}, E_{6}\right)}$. The number in the bracket denotes the self-intersection number of the curve. The letter denotes an intersection curve with the corresponding divisor. The "/" symbol means that the curves are in the same homology class. The assignment of ruling/section on each surface component is denoted by blue/red colors.

the requirement that each intersection curve $S_{i} \cdot S_{j}$ is a ruling/section on both $S_{i}$ and $S_{j}$. We list the rational ruling curves with self-intersection 0 on each surface component:

$$
\begin{aligned}
& S_{1}: f^{(1)} \equiv u_{2}=\delta_{4}, \\
& S_{2}: f^{(2)} \equiv u_{5}=v_{6}+\delta_{4}+\delta_{5}, \\
& S_{3}: f^{(3)} \equiv U=\delta_{5}, \\
& S_{4}: f^{(4)} \equiv \delta_{2}=u_{3}+\delta_{1}+v_{3}, \\
& S_{5}: f^{(5)} \equiv u_{3}=u_{7}+\delta_{2}=u_{1}+\delta_{3}+v_{1} .
\end{aligned}
$$

Hence we conclude that this geometry describes a quiver gauge theory with gauge groups $\mathrm{SU}(3) \times \mathrm{SU}(2)^{(1)} \times \mathrm{SU}(2)^{(2)} \times \mathrm{SU}(2)^{(3)}$. The Cartan divisors of the $\mathrm{SU}(3)$ factor correspond to the surface components $S_{4}$ and $S_{5}$, while the Cartan divisors of $\mathrm{SU}(2)^{(1)}$, $\mathrm{SU}(2)^{(2)}$ and $\mathrm{SU}(2)^{(3)}$ correspond to the surface components $S_{1}, S_{2}$ and $S_{3}$ respectively.

The matter fields of this quiver gauge theory can be read off from the $(-1)$-curves in figure 42 that are a part of ruling (colored by blue). Their representations under SU(3) $\times$ $\mathrm{SU}(2)^{(1)} \times \mathrm{SU}(2)^{(2)} \times \mathrm{SU}(2)^{(3)}$ are:

$$
\begin{array}{ll}
v_{6} \cdot S_{2}: & (\mathbf{3}, \mathbf{1}, \mathbf{2}, \mathbf{1}) \\
S_{2} \cdot S_{5}: & (\overline{\mathbf{3}}, \mathbf{1}, \mathbf{2}, \mathbf{1}) \\
u_{3} \cdot S_{4}: & (\mathbf{3}, \mathbf{2}, \mathbf{1}, \mathbf{1}) \\
v_{3} \cdot S_{4}: & (\overline{\mathbf{3}}, \mathbf{2}, \mathbf{1}, \mathbf{1}) \\
u_{1} \cdot S_{5}: & (\mathbf{3}, \mathbf{1}, \mathbf{1}, \mathbf{2}) \\
v_{1} \cdot S_{5}: & (\overline{\mathbf{3}}, \mathbf{1}, \mathbf{1}, \mathbf{2}) \\
u_{7} \cdot S_{5}: & (\mathbf{3}, \mathbf{1}, \mathbf{2}, \mathbf{1})
\end{array}
$$


Thus we conclude that the quiver description is:

$$
\begin{gathered}
\mathrm{SU}(2) \\
\mathrm{SU}(2)-\mathrm{SU}(3)-\mathrm{SU}(2),
\end{gathered}
$$

since all the matter fields in (C.34) are bifundamentals of this quiver gauge theory.

From the geometry in figure 42 , we can blow up the surfaces $S_{1}, S_{2}$ and $S_{3}$ to get the geometry corresponding to the marginal CFD:
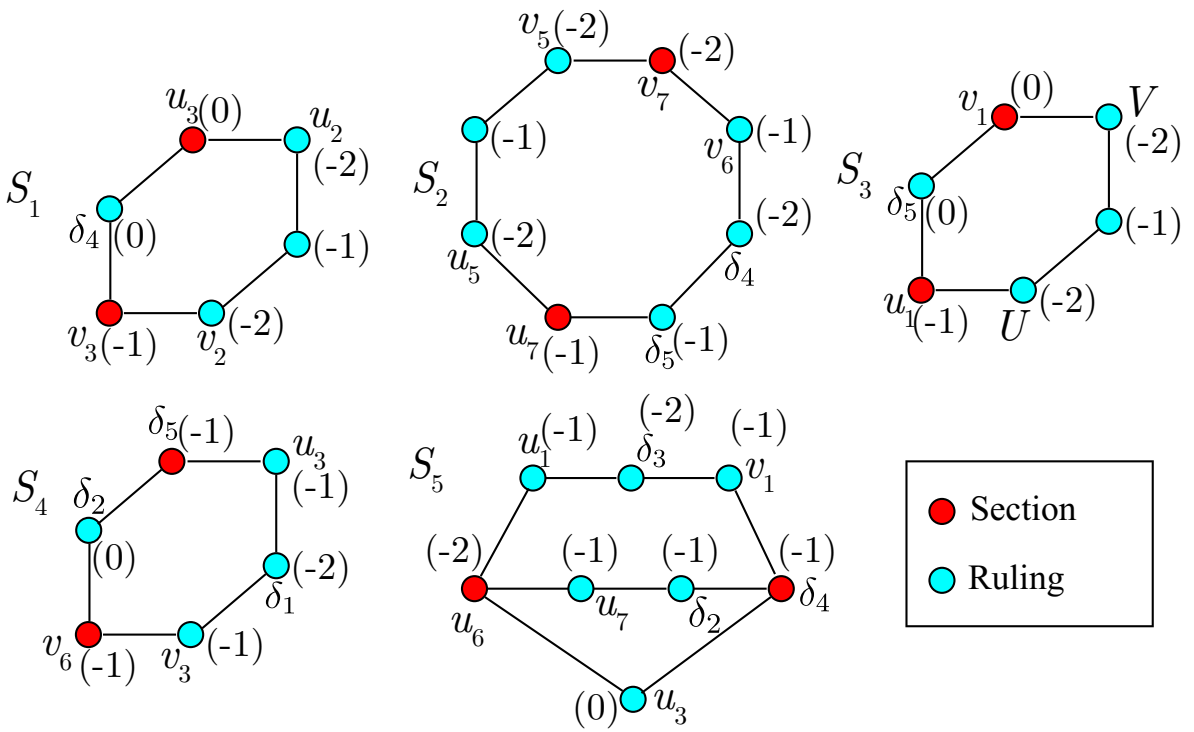

The quiver gauge theory description of this geometry is

$$
[2]-\mathrm{SU}(2)-\mathrm{SU}(3)-\mathrm{SU}(2)-[2],
$$

and the removal of fundamental flavors charged under the three $\mathrm{SU}(2) \mathrm{s}$ will exactly correspond to shrinking the unlabeled (-1)-curves on $S_{1}, S_{2}$ and $S_{3}$ in (C.36).

As a summary, we confirmed that the proposed CFD tree with the star-shaped $\mathrm{SU}(3) \times \mathrm{SU}(2) \times \mathrm{SU}(2) \times \mathrm{SU}(2)$ quiver in $[1,2]$ is indeed backed up with a solid Calabi-Yau threefold geometry.

\section{C.3 Marginal geometry for $\left(E_{7}, \mathrm{SO}(7)\right)$ minimal conformal matter}

In this section we present a marginal geometry of minimal $\left(E_{7}, \mathrm{SO}(7)\right)$ conformal matter that manifestly has the two dual gauge descriptions $\mathrm{SU}(4)_{0}+2 \boldsymbol{A} \boldsymbol{S}+8 \boldsymbol{F}$ and $6 \boldsymbol{F}-\mathrm{Sp}(2)-$ $\mathrm{Sp}(1)-2 \boldsymbol{F}$. The codimension one affine fibers of $E_{7}$ and $\mathrm{SO}(7)$ are labelled as:

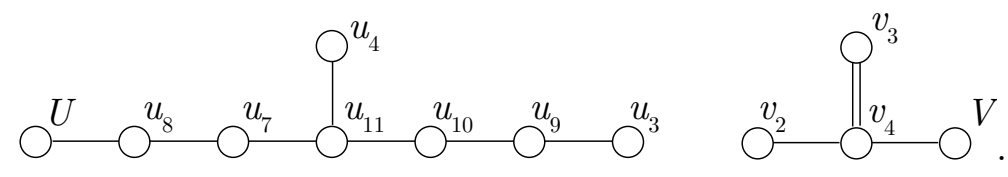



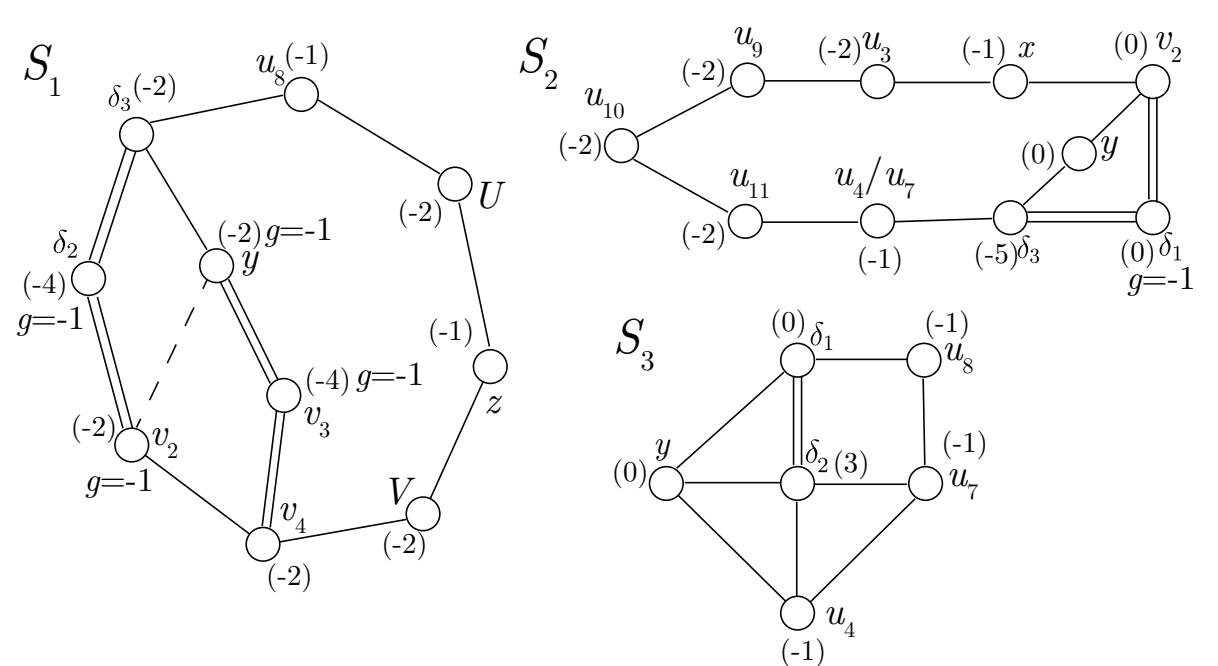

Figure 43. The configuration of curves $S_{j} \cap\{d=0\} \equiv d$ on the compact surfaces $S_{j}=$ $\left\{\delta_{j}=0\right\}(j=1, \ldots, 3)$ in the marginal $\left(E_{7}, \mathrm{SO}(7)\right)$ geometry, where $\{d=0\}$ are (possibly noncompact) divisors in the resolved Calabi-Yau threefold. The number in the bracket denotes the selfintersection number of the curve. There are reducible curves $C \subset S$ that satisfy $C \cdot\left(K_{S}+C\right)=-4$, which we formally label as curves with genus $g=-1$. All other curves have genus 0 . The dashed line in $S_{1}$ indicates an intersection number of -1 ; this reflects the fact that the two involved curves, which are reducible, share irreducible components. The precise structure of these irreducible components are, however, immaterial to our discussion here.

Note that locally, the fiber of $\left\{v_{3}=0\right\}$ is formed by two disconnected $\mathbb{P}^{1} \mathrm{~s}$, which are identified via monodromy effects and reflect the folding of $\mathrm{SO}(8)$ to $\mathrm{SO}(7)$. Thus, the condition for $\left\{v_{3}\right\}$ to be fully wrapped requires the self-intersection inside the three compact surfaces $\mathcal{S}=\bigcup_{j=1}^{3} S_{j}$ to be -4 , rather than -2 . Concretely, the curves on the $S_{j}$ 's are show in figure 43 .

The rulings $f_{j}^{\mathrm{SU}(4)} \hookrightarrow S_{j}$ giving rise to the $\mathrm{SU}(4)$ gauge description are given by the curves

$$
\begin{aligned}
f_{1}^{\mathrm{SU}(4)} & \equiv z+U+u_{8}=y+v_{3}+v_{4}, \\
f_{2}^{\mathrm{SU}(4)} & \equiv y=u_{4}+u_{11}+u_{10}+u_{9}+u_{3}+x, \\
f_{3}^{\mathrm{SU}(4)} & \equiv y=u_{8}+u_{7},
\end{aligned}
$$

where the equalities are understood as rational equivalence relations on each surface. To realize the $\mathrm{SU}(4)$, the surfaces are glued as $S_{1}-S_{3}-S_{2}$. Each gluing curve is a 1-section with respect to the rulings (C.39): we have

$$
\left.\left(\delta_{3} \cdot f_{1}^{\mathrm{SU}(4)}\right)\right|_{S_{1}}=\left.\left(\delta_{1} \cdot f_{3}^{\mathrm{SU}(4)}\right)\right|_{S_{3}}=\left.\left(\delta_{2} \cdot f_{3}^{\mathrm{SU}(4)}\right)\right|_{S_{3}}=\left.\left(\delta_{3} \cdot f_{2}^{\mathrm{SU}(4)}\right)\right|_{S_{2}}=1 .
$$

The rulings $f_{j}^{\text {quiver }} \hookrightarrow S_{j}$ realizing the $\operatorname{Sp}(2) \times \operatorname{Sp}(1)$ quiver are

$$
\begin{aligned}
& f_{1}^{\text {quiver }} \equiv U+2 z+V=\delta_{3}+\delta_{2}+v_{2}, \\
& f_{2}^{\text {quiver }} \equiv y=u_{4}+u_{11}+u_{10}+u_{9}+u_{3}+x=f_{2}^{\mathrm{SU}(4)}, \\
& f_{3}^{\text {quiver }} \equiv \delta_{1}=u_{4}+u_{7} .
\end{aligned}
$$


The $\operatorname{Sp}(2)$ is supported on $S_{2}-S_{3}$. The non-simply laced nature is reflected by the intersection numbers of the gluing curves with these rulings:

$$
\left.\left(\delta_{3} \cdot f_{2}^{\text {quiver }}\right)\right|_{S_{2}}=1,\left.\quad\left(\delta_{2} \cdot f_{3}^{\text {quiver }}\right)\right|_{S_{3}}=2 .
$$

To have an consistent, independent $\operatorname{Sp}(1)$ factor on $S_{1}$, the gluing curves $S_{1} \cap S_{2}$ and $S_{1} \cap S_{3}$ need to be fibers on all three surfaces, which indeed is the case.

These two gauge descriptions can be verified by matching the prepotentials with the cubic intersection numbers,

$$
\begin{array}{rrrr}
S_{1}^{3}=2, & S_{1}^{2} \cdot S_{2}=0 & S_{1} \cdot S_{2}^{2}=-4, & S_{1}^{2} \cdot S_{3}=0,
\end{array}
$$

Open Access. This article is distributed under the terms of the Creative Commons Attribution License (CC-BY 4.0), which permits any use, distribution and reproduction in any medium, provided the original author(s) and source are credited.

\section{References}

[1] F. Apruzzi, C. Lawrie, L. Lin, S. Schäfer-Nameki and Y.-N. Wang, 5d Superconformal Field Theories and Graphs, Phys. Lett. B 800 (2020) 135077 [arXiv:1906.11820] [INSPIRE].

[2] F. Apruzzi, C. Lawrie, L. Lin, S. Schäfer-Nameki and Y.-N. Wang, Fibers add Flavor. Part I. Classification of 5d SCFTs, Flavor Symmetries and BPS States, JHEP 11 (2019) 068 [arXiv: 1907.05404] [INSPIRE].

[3] N. Seiberg, Five-dimensional SUSY field theories, nontrivial fixed points and string dynamics, Phys. Lett. B 388 (1996) 753 [hep-th/9608111] [INSPIRE].

[4] E. Witten, Some comments on string dynamics, in Future perspectives in string theory. Proceedings, Conference, Strings'95, Los Angeles, U.S.A., 13-18 March 1995, pp. 501-523 (1995) [hep-th/9507121] [INSPIRE].

[5] J.J. Heckman, D.R. Morrison and C. Vafa, On the Classification of 6D SCFTs and Generalized ADE Orbifolds, JHEP 05 (2014) 028 [Erratum ibid. 06 (2015) 017] [arXiv: 1312.5746] [INSPIRE].

[6] J.J. Heckman, D.R. Morrison, T. Rudelius and C. Vafa, Atomic Classification of 6D SCFTs, Fortsch. Phys. 63 (2015) 468 [arXiv:1502.05405] [INSPIRE].

[7] L. Bhardwaj, Classification of $6 d \mathcal{N}=(1,0)$ gauge theories, JHEP 11 (2015) 002 [arXiv: 1502.06594] [INSPIRE].

[8] M. Del Zotto, J.J. Heckman and D.R. Morrison, $6 D$ SCFTs and Phases of $5 D$ Theories, JHEP 09 (2017) 147 [arXiv: 1703.02981] [INSPIRE].

[9] P. Jefferson, H.-C. Kim, C. Vafa and G. Zafrir, Towards Classification of 5d SCFTs: Single Gauge Node, arXiv:1705.05836 [INSPIRE].

[10] P. Jefferson, S. Katz, H.-C. Kim and C. Vafa, On Geometric Classification of $5 d$ SCFTs, JHEP 04 (2018) 103 [arXiv: 1801.04036] [INSPIRE].

[11] C. Closset, M. Del Zotto and V. Saxena, Five-dimensional SCFTs and gauge theory phases: an M-theory/type IIA perspective, SciPost Phys. 6 (2019) 052 [arXiv:1812.10451] [INSPIRE]. 
[12] F. Apruzzi, L. Lin and C. Mayrhofer, Phases of $5 d$ SCFTs from M-/F-theory on Non-Flat Fibrations, JHEP 05 (2019) 187 [arXiv:1811.12400] [INSPIRE].

[13] L. Bhardwaj and P. Jefferson, Classifying 5d SCFTs via 6d SCFTs: Rank one, JHEP 07 (2019) 178 [arXiv: 1809.01650] [INSPIRE].

[14] L. Bhardwaj and P. Jefferson, Classifying 5d SCFTs via 6d SCFTs: Arbitrary rank, JHEP 10 (2019) 282 [arXiv: 1811.10616] [INSPIRE].

[15] O. Aharony and A. Hanany, Branes, superpotentials and superconformal fixed points, Nucl. Phys. B 504 (1997) 239 [hep-th/9704170] [INSPIRE].

[16] O. Aharony, A. Hanany and B. Kol, Webs of $(p, q)$ five-branes, five-dimensional field theories and grid diagrams, JHEP 01 (1998) 002 [hep-th/9710116] [INSPIRE].

[17] O. DeWolfe, A. Hanany, A. Iqbal and E. Katz, Five-branes, seven-branes and five-dimensional $E_{n}$ field theories, JHEP 03 (1999) 006 [hep-th/9902179] [INSPIRE].

[18] A. Brandhuber and Y. Oz, The D4-D8 brane system and five-dimensional fixed points, Phys. Lett. B 460 (1999) 307 [hep-th/9905148] [INSPIRE].

[19] O. Bergman and D. Rodriguez-Gomez, 5d quivers and their AdS $S_{6}$ duals, JHEP 07 (2012) 171 [arXiv: 1206.3503] [INSPIRE].

[20] O. Bergman and G. Zafrir, 5d fixed points from brane webs and O7-planes, JHEP 12 (2015) 163 [arXiv: 1507.03860] [INSPIRE].

[21] G. Zafrir, Brane webs, 5d gauge theories and $6 d \mathcal{N}=(1,0)$ SCFT's, JHEP 12 (2015) 157 [arXiv: 1509.02016] [INSPIRE].

[22] G. Zafrir, Brane webs and O5-planes, JHEP 03 (2016) 109 [arXiv:1512.08114] [INSPIRE].

[23] K. Ohmori and H. Shimizu, $S^{1} / T^{2}$ compactifications of $6 d \mathcal{N}=(1,0)$ theories and brane webs, JHEP 03 (2016) 024 [arXiv: 1509.03195] [inSPIRE].

[24] H. Hayashi, S.-S. Kim, K. Lee and F. Yagi, Discrete theta angle from an O5-plane, JHEP 11 (2017) 041 [arXiv: 1707.07181] [INSPIRE].

[25] H. Hayashi, S.-S. Kim, K. Lee and F. Yagi, 5-brane webs for $5 d \mathcal{N}=1 G_{2}$ gauge theories, JHEP 03 (2018) 125 [arXiv:1801.03916] [INSPIRE].

[26] H. Hayashi, S.-S. Kim, K. Lee and F. Yagi, Dualities and 5-brane webs for $5 d$ rank 2 SCFTs, JHEP 12 (2018) 016 [arXiv:1806.10569] [INSPIRE].

[27] H.-C. Kim, S.-S. Kim and K. Lee, 5-dim Superconformal Index with Enhanced $E_{n}$ Global Symmetry, JHEP 10 (2012) 142 [arXiv:1206.6781] [INSPIRE].

[28] O. Bergman, D. Rodríguez-Gómez and G. Zafrir, 5-Brane Webs, Symmetry Enhancement and Duality in 5d Supersymmetric Gauge Theory, JHEP 03 (2014) 112 [arXiv:1311.4199] [INSPIRE].

[29] G. Zafrir, Duality and enhancement of symmetry in 5d gauge theories, JHEP 12 (2014) 116 [arXiv: 1408.4040] [INSPIRE].

[30] V. Mitev, E. Pomoni, M. Taki and F. Yagi, Fiber-Base Duality and Global Symmetry Enhancement, JHEP 04 (2015) 052 [arXiv:1411.2450] [INSPIRE].

[31] C. Hwang, J. Kim, S. Kim and J. Park, General instanton counting and 5d SCFT, JHEP 07 (2015) 063 [arXiv: 1406.6793] [INSPIRE]. 
[32] Y. Tachikawa, Instanton operators and symmetry enhancement in 5d supersymmetric gauge theories, PTEP 2015 (2015) 043B06 [arXiv:1501.01031] [INSPIRE].

[33] K. Yonekura, Instanton operators and symmetry enhancement in 5d supersymmetric quiver gauge theories, JHEP 07 (2015) 167 [arXiv:1505.04743] [INSPIRE].

[34] D. Gaiotto and H.-C. Kim, Duality walls and defects in $5 d \mathcal{N}=1$ theories, JHEP 01 (2017) 019 [arXiv: 1506.03871] [INSPIRE].

[35] G. Zafrir, Instanton operators and symmetry enhancement in 5d supersymmetric USp, SO and exceptional gauge theories, JHEP 07 (2015) 087 [arXiv: 1503.08136] [INSPIRE].

[36] O. Bergman and D. Rodriguez-Gomez, A Note on Instanton Operators, Instanton Particles and Supersymmetry, JHEP 05 (2016) 068 [arXiv:1601.00752] [INSPIRE].

[37] G. Ferlito, A. Hanany, N. Mekareeya and G. Zafrir, 3d Coulomb branch and $5 d$ Higgs branch at infinite coupling, JHEP 07 (2018) 061 [arXiv:1712.06604] [INSPIRE].

[38] S. Cabrera, A. Hanany and F. Yagi, Tropical Geometry and Five Dimensional Higgs Branches at Infinite Coupling, JHEP 01 (2019) 068 [arXiv:1810.01379] [INSPIRE].

[39] D.R. Morrison and N. Seiberg, Extremal transitions and five-dimensional supersymmetric field theories, Nucl. Phys. B 483 (1997) 229 [hep-th/9609070] [INSPIRE].

[40] K.A. Intriligator, D.R. Morrison and N. Seiberg, Five-dimensional supersymmetric gauge theories and degenerations of Calabi-Yau spaces, Nucl. Phys. B 497 (1997) 56 [hep-th/9702198] [INSPIRE].

[41] M.R. Douglas, S.H. Katz and C. Vafa, Small instantons, Del Pezzo surfaces and type-I-prime theory, Nucl. Phys. B 497 (1997) 155 [hep-th/9609071] [INSPIRE].

[42] D. Xie and S.-T. Yau, Three dimensional canonical singularity and five dimensional $\mathcal{N}=1$ SCFT, JHEP 06 (2017) 134 [arXiv:1704.00799] [INSPIRE].

[43] C. Lawrie and S. Schäfer-Nameki, The Tate Form on Steroids: Resolution and Higher Codimension Fibers, JHEP 04 (2013) 061 [arXiv: 1212.2949] [INSPIRE].

[44] H. Hayashi, C. Lawrie, D.R. Morrison and S. Schäfer-Nameki, Box Graphs and Singular Fibers, JHEP 05 (2014) 048 [arXiv: 1402 . 2653] [INSPIRE].

[45] C. Vafa, Evidence for F-theory, Nucl. Phys. B 469 (1996) 403 [hep-th/9602022] [InSPIRE].

[46] D.R. Morrison and C. Vafa, Compactifications of F-theory on Calabi-Yau threefolds. 1, Nucl. Phys. B 473 (1996) 74 [hep-th/9602114] [INSPIRE].

[47] D.R. Morrison and C. Vafa, Compactifications of F-theory on Calabi-Yau threefolds. 2., Nucl. Phys. B 476 (1996) 437 [hep-th/9603161] [INSPIRE].

[48] M. Del Zotto, J.J. Heckman, A. Tomasiello and C. Vafa, 6d Conformal Matter, JHEP 02 (2015) 054 [arXiv: 1407.6359] [INSPIRE].

[49] A.P. Braun and S. Schäfer-Nameki, Box Graphs and Resolutions I, Nucl. Phys. B 905 (2016) 447 [arXiv: 1407.3520] [INSPIRE].

[50] A.P. Braun and S. Schäfer-Nameki, Box Graphs and Resolutions II: From Coulomb Phases to Fiber Faces, Nucl. Phys. B 905 (2016) 480 [arXiv:1511.01801] [InSPIRE].

[51] C. Lawrie, S. Schäfer-Nameki and J.-M. Wong, F-theory and All Things Rational: Surveying U(1) Symmetries with Rational Sections, JHEP 09 (2015) 144 [arXiv: 1504.05593] [INSPIRE]. 
[52] T.W. Grimm and H. Hayashi, F-theory fluxes, Chirality and Chern-Simons theories, JHEP 03 (2012) 027 [arXiv: 1111.1232] [INSPIRE].

[53] S. Krause, C. Mayrhofer and T. Weigand, $G_{4}$ flux, chiral matter and singularity resolution in F-theory compactifications, Nucl. Phys. B 858 (2012) 1 [arXiv:1109.3454] [InSPIRE].

[54] J. Marsano and S. Schäfer-Nameki, Yukawas, G-flux and Spectral Covers from Resolved Calabi-Yau's, JHEP 11 (2011) 098 [arXiv:1108.1794] [INSPIRE].

[55] S. Schäfer-Nameki and T. Weigand, F-theory and 2d (0,2) theories, JHEP 05 (2016) 059 [arXiv: 1601.02015] [INSPIRE].

[56] W. Fulton and J. Harris, Representation theory, Graduate Texts in Mathematics, vol. 129, Springer-Verlag, New York (1991) [DOI].

[57] M. Esole and S.-T. Yau, Small resolutions of SU(5)-models in F-theory, Adv. Theor. Math. Phys. 17 (2013) 1195 [arXiv:1107.0733] [INSPIRE].

[58] H. Hayashi, C. Lawrie and S. Schäfer-Nameki, Phases, Flops and F-theory: SU(5) Gauge Theories, JHEP 10 (2013) 046 [arXiv: 1304.1678] [INSPIRE].

[59] M. Esole, S.-H. Shao and S.-T. Yau, Singularities and Gauge Theory Phases, Adv. Theor. Math. Phys. 19 (2015) 1183 [arXiv:1402.6331] [InSPIRE].

[60] M. Esole, S.-H. Shao and S.-T. Yau, Singularities and Gauge Theory Phases II, Adv. Theor. Math. Phys. 20 (2016) 683 [arXiv:1407.1867] [InSPIRE].

[61] M. Esole, R. Jagadeesan and M.J. Kang, 48 Crepant Paths to $\mathrm{SU}(2) \times \mathrm{SU}(3)$, arXiv: 1905.05174 [INSPIRE].

[62] M. Esole, M.J. Kang and S.-T. Yau, Mordell-Weil Torsion, Anomalies and Phase Transitions, arXiv:1712.02337 [INSPIRE].

[63] M. Esole, R. Jagadeesan and M.J. Kang, The Geometry of $G_{2}$, Spin(7) and $\operatorname{Spin}(8)$-models, arXiv: 1709.04913 [INSPIRE].

[64] M. Esole and M.J. Kang, Flopping and Slicing: SO(4) and Spin(4)-models, Adv. Theor. Math. Phys. 23 (2019) 1003 [arXiv:1802.04802] [INSPIRE].

[65] M. Esole and M.J. Kang, The Geometry of the $\mathrm{SU}(2) \times G_{2}$-model, JHEP 02 (2019) 091 [arXiv: 1805.03214] [INSPIRE].

[66] M. Esole, P. Jefferson and M.J. Kang, The Geometry of F-Models, arXiv:1704.08251 [INSPIRE].

[67] M. Esole and S. Pasterski, $D_{4}$-flops of the $E_{7}$-model, arXiv:1901.00093 [INSPIRE].

[68] L. Bhardwaj and Y. Tachikawa, Classification of $4 d N=2$ gauge theories, JHEP 12 (2013) 100 [arXiv: 1309.5160] [INSPIRE].

[69] N. Mekareeya, K. Ohmori, Y. Tachikawa and G. Zafrir, E8 instantons on type-A ALE spaces and supersymmetric field theories, JHEP 09 (2017) 144 [arXiv: 1707.04370] [INSPIRE].

[70] G. Zafrir, private communication.

[71] L. Bhardwaj, Dualities of 5d gauge theories from S-duality, arXiv:1909.05250 [INSPIRE]. 